WSRC-RP-92-878

DE93 001894

\title{
SOIL GAS INVESTIGATIONS AT THE SANITARY LANDFILL (U)
}

by

D. E. Wyatt, R. J. Pirkle, and D. J. Masdea

Westinghouse Savannah River Company'

Savannah River Site

Aiken, South Carolina 29808

This report was prepared in connection with work done under Contract No. DE-AC09-89SR18035 with the U.S. Department of Energy. By acceptance of this report, the publisher and/or recipient acknowledges the U.S. Government's right to retain a nonexclusive, royalty-free license in and to any copyright covering this report, along with the right to reproduce and to authorize others to reproduce all or part of the copyrighted report.
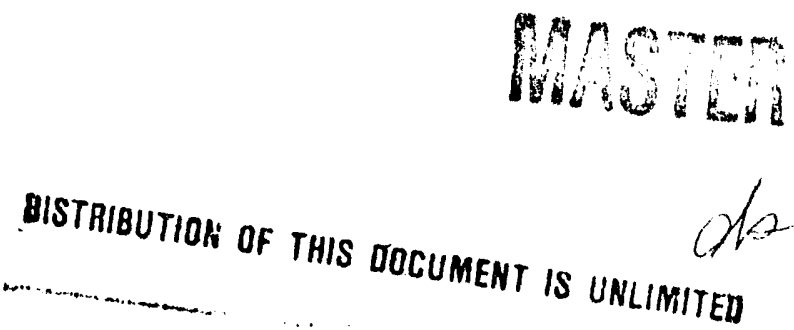


\title{
Soil Gas Investigations at the Sanitary Landfill (U)
}

\author{
D. E. Wyatt \\ R. J. Pirkle \\ D. J. Masdea
}

Westinghouse Savannah River Company Savannah River Site

Aiken, SC 29808 


\section{DISCLAIMER}

This report was prepared by Westinghouse Savannah River Company (WSRC) for the United States Department of Energy under Contract No. DE-AC09-89SR18035 and is an account of work performed under that contract. Neither the United States Department of Energy, nor WSRC, nor any of their employees makes any warranty, express or implied, or assumes any legal liability or responsibility for the accuracy, completeness, or usefulness, of any information, apparatus, or product or process disclosed herein or represents that its use will not infringe privately owned rights. Reference herein to any specific commercial product, process, or service by trademark, name, manufacturer or otherwise does not necessarily constitute or imply endorsement, recommendation, or favoring of same by WSRC or by the United States Government or any agency therefof. The views and opinions of the authors expressed herein do not necessarily state or reflect those of the United States Government or any agency thereof.

Printed in the United States of America

Available from

National Technical Information Service

U. S. Department of Commerce

5285 Port Royal Road

Springfield, VA 22161 
WSRC-RP-92-878

\section{Soil Gas Investigations at the Sanitary Landfill (U)}

D. E. Wyatt

R. J. Pirkle

D. J. Masdea

Westinghouse Savannah River Company

Savannah River Site

Aiken, SC 29808 


\section{TABLE OF CONTENTS}

List of Figures $\quad 2$

$\begin{array}{ll}\text { List } \text { of Tables } & 6\end{array}$

$\begin{array}{ll}\text { Executive Summary } & 7\end{array}$

$\begin{array}{ll}\text { I. Introduction } & 8\end{array}$

$\begin{array}{ll}\text { II. Background } & 8\end{array}$

III. Quality Assurance 9

A. Sampling Methods 9

B. Analyticil Methods 9

$\begin{array}{ll}\text { C. Accuracy } & 10\end{array}$

D. Reliability of Selected Data 10

$\begin{array}{ll}\text { E. Precision } & 11\end{array}$

F. Minimum Detection Levels $\quad 12$

$\begin{array}{ll}\text { G. Blanks } & 13\end{array}$

H. Data on Magnetic Disk 13

$\begin{array}{ll}\text { IV. Results } & 14\end{array}$

$\begin{array}{ll}\text { V. Conclusions } & 17\end{array}$

VI. Appendix I

Sampling Method SM1

Sampling Method SM4

Analytical Method AMI

Analytical Method AM4

Analytical Method AME

Standard Preparation Method SP3

VII. Appendix II

Soil Gas Collection Logs

Mercury Collection Logs

Waste Management Unit Worksheet 


\section{LIST OF FIGURES}

Figure la. The 740-G Sanitary Landfill Site Index Map (all locations@ @ 1"=400 ft)

Figure Ib. The 740-G Sanitary Landfill North Area Sample Location Map (sample locations@1"=250 ft)

Figure Ic. The 740-G Sanitary Landfill South Area Sample Location Map (sample locations@1"=250 it)

Figure Ic. The 740-G Sanitary Landfill Background Area Sample Location Map (sample locations @ 1" = $200 \mathrm{ft}$ )

Figure 2a. Gas Chromatogram of Soil Gases at Site 111 from the Flame Ionization Detector(FID)

Figure 2b. Gas Chromatogram of Soil Gases at Site 111 from the Electron Capture Detector(ECD)

Figure 3. Gas Chromatogram of System Blank \# 7

Figure 4. Gas Chromatogram of $\mathrm{C}_{1}-\mathrm{C}_{4}$ Standards " $\mathrm{M}$ " and "224"

Figure 5. Gas Chromatogram (FID) of VOC Standard "I", Level 3

Figure 6. Gas Chromatogram (ECD) of VOC Standard "I", Level 4

Figure 7. Benzene Retention Time vs Room Temperature

Figure 8. Gas Chromatogram of $\mathrm{C}_{1}-\mathrm{C}_{4}$ Hydrocarbon Standard M @ 200:1 Dilution in N2

Figure 9. Methane Symbol Map

Figure 10. Methane (percent) Map, North Area

Figure 11. Methane (percent) Map, South Area

Figure 12. Propane Symbol Map

Figure 13. Propane (ppmv) Map, North Area

Figure 14. Propane (ppmv) Map, South Area

Figure 15. n-Butane Symbol Map 
LIST OE FIGURES (cont.)

Figure 16. n-Butane (ppmv) Map, North Area

Figure 17. n-Butane (ppmv) Map, South Area

Figure 18. i-Butane Symbol Map

Figure 19. i-Butane (ppmv) Map, North Area

Figure 20. i-Butane (ppmv) Map, South Area

Figure 21. Pentane Symbol Map

Figure 22. Pentane (ppmv) Map, North Area

Figure 23. Pentane (ppmv) Map, South Area

Figure 24. Hexane Symbol Map

Figure 25. Hexane (ppmv) Map, North Area

Figure 26. Hexane (ppmv) Map, South Area

Figure 27. Heptane Symbol Map

Figure 28. Heptane (ppmv) Map, North Area

Figure 29. Heptane (ppmv) Map, South Area

Figure 30. Octane Symbol Map

Figure 31. Octane (ppmv) Map, North Area

Figure 32. Octane (ppmv) Map, South Area

Figure 33. Nonane Symbol Map

Figure 34. Nonane (ppn. ') Map, North Area

Figure 35. Nonane (ppmv) Map, South Area

Figure 36. Decane Symbol Map

Figure 37. Decane (ppmv) Map, North Area

Figure 38. Decane (ppmv) Map, South Area 


\section{LIST OF EJGURES (cont.)}

Figure 39. Ethylene Symbol Map

Figure 40. Ethylene (ppmv) Map, North Area

Figure 41. Ethylene (ppmv) Map, South Area

Figure 42. Propylene Symbol Map

Figure 43. Propylene (ppmv) Map, North Area

Figure 44. Propylene (ppmv) Map, South Area

Figure 45. Benzene Symbol Map

Figure 46. Benzene (ppmv) Map, North Area

Figure 47. Benzene (ppmv) Map, South Area

Figure 48. Toluene Symbol Map

Figure 49. Toluene (ppmv) Map, North Area

Figure 50. Toluene (ppmv) Map, South Area

Figure 51. Ethylbenzene Symbol Map

Figure 52. Ethylbenzene (ppmv) Map, North Area

Figure 53. Ethylbenzene (ppmv) Map, South Area

Figure 54. m- \& p-Xylene Symbol Map

Figure 55. m- \& p-Xylene (ppmv) Map, North Area

Figure 56. m- \& p-Xylene (ppmv) Map, South Area

Figure 57. o-Xylene Symbol Map

Figure 58. o-Xylene (ppmv) Map, North Area

Figure 59. o-Xylene (ppmv) Map, South Area

Figure 60. Methylene Chloride Symbol Map

Figure 61. Methylene Chloride (ppmv) Map, North Area 


\section{LIST OF FIGURES (cont.)}

Figure 62. Methylene Chloride (ppmv) Map, South Area

Figure 63. Chloroform Symbol Map

Figure 64. Chloroform (ppmv) Map, North Area

Figure 65. Chloroform (ppmv) Map, South Area

Figure 66. trans 1,2-Dichloroethylene Symbol Map

Figure 67. trans 1,2-Dichloroethylene (ppmv) Map, North Area

Figure 68. trans 1,2-Dichloroethylene (ppmv) Map, South Area

Figure 69. 1,1,1-Trichloroethane Symbol Map

Figure 70. 1,1,1-Trichloroethane (ppmv) Map, North Area

Figure 71. 1,1,1-Trichloroethane (ppmv) Map, South Area

Figure 72. Trichloroethylene Symbol Map

Figure 73. Trichloroethylene (ppmv) Map, North Area

Figure 74. Trichloroethylene (ppmv) Map, South Area

Figure 75. Tetrachloroethylene Symbol Map

Figure 76. Tetrachloroethylene (ppmv) Map, North Area

Figure 77. Tetrachloroethylene (ppmv) Map, South Area

Figure 78. Mercury Symbol Map

Figure 79. Mercury (ng/g) Map 


\section{LIST OE TABLES}

Table 1. Hydrocarbon Soil Gas Concentrations and System Blanks at the 740-G Sanitary Landfill

Table 2. Chlorinated Hydrocarbon Soil Gas Concentrations and System Blanks at the 740-G Sanitary Landfill

Table 3. Light Hydrocarbon Soil Gas, Soil Mercury Concentrations and System Blanks at the 740-G Sanitary Landfill

Table 4. VOC Prepared Standard Concentrations

Table 5. Analyses of "l" Standards: a) Hydrocarbons; b) Chlorinated Hydrocarbons

Table 6. Analyses of "I2" Standards: a) Hydrocarbons; b) Chlorinated Hydrocarbons

Table 7. Analyses of "J2" Standards: a) Hydrocarbons; b) Chlorinated Hydrocarbons

Table 8. Light Hydrocarbon Analyses of All Standards "M" and "224"

Table 9. Minimum Detection Levels

Table 10. System Blanks for All Sanitary Landfill Data Directories: a) Hydrocarbons; b) Chlorinated Hydrocarbons 
A soil gas survey was performed at the 740-G Sanitary Landfill during December, 1990. The survey monitored the presence and distribution of the $\mathrm{C}_{\mathrm{I}}$ $\mathrm{C}_{4}$ hydrocarbons; the $\mathrm{C}_{5}-\mathrm{C}_{10}$ normal paraffins; the aromatic hydrocarbons, BTXE; selected chlorinated hydrocarbons; and mercury. Significant levels of several of these contaminants were found associated with the burial site. In the northern area of the Landfill, methane concentrations ranged up to $63 \%$ of the soil gas and were consistently high on the western side of the access road. To the east of the access road in the northern and southern area high concentrations of methane were enccuntered but were not consistently high. Methane, the species found in highest concentration in the landfill, was generated in the landfill as the result of biological oxidation of cellulose and other organics to carbon dioxide followed by reduction of the carbon dioxide to methane. Distributions of other species are the result of burials in the landfill of solvents or other materials. 


\section{INTRODUCTION}

A soil gas survey was designed and executed at the 740-G, Sanitary Landfill at the Savannah River Site in December, 1990. The survey was extended in May, 1991. The objective of the survey was to determine the presence and extent, or absence of, contamination in near surface soil gases. A sample grid on 100 foot centers consisting of 288 sample locations was established, as shown on Figures la-d, and sampled for soil gases in order to accomplish these objectives. The outermost row of samples along the eastern, western and southern boundaries of the grid over both the north and south areas of the landfill, as shown on Figures $\mathrm{lb}$ and $\mathrm{IC}$, is approximately 100 feet outside the area of burial and was designed to provide evidence of migration of monitored species from the landfill. Along the southern boundary of the landfill a second row of boundary sites was established 10 feet to the south of the first boundary rows shown on Figure lc. This second row was sampled at a depth of 6 feet (as compared to 3 feet at all other sites). Species monitored were the light hydrocarbons, $\left(C_{1}-C_{4}\right)$; gasoline range normal paraffins $\left(C_{5}-C_{10}\right)$; gasoline range aromatic hydrocarbons, (BTXE); and selected chlorinated organics. Soil samples were also taken at sites 1-261 and analyzed for labile mercury. Analyses of soil and soil gas samples were carried out in an on-site laboratory. The results of all analyses are shown on Tables 1,2 and 3, and selected data are mapped as shown on Figures $9-79$.

\section{BACKGROUND}

The 740-G Sanitary Landfill was established in 1974 and remains active at the present. The dimensions are $3,275 \times 1300 \mathrm{ft}$ or about 70 acres. Burial has been in excavated earthen trenches and the known disposal at this site as documented on page A-12 of Volume I: Waste Management Unit Worksheets, October, 1990; WSRC-RP90-1046 (which is atiached in Appendix II), includes paper, plastic, construction and metallic debris, solvent rags, empty cans, and carcasses. At the time this soil gas survey was begun, low levels of volatile organic compounds, including trichloroethylene, tetrachloroethylene, and 1,1,1trichloroethane had been reported in groundwater monitoring wells at the site. 


\section{OUALITY ASSURANCE}

The quality assurance/quality control procedures of the sampling subcontractor were followed for all field and laboratory analytical work. Documentation of applicable sampling and analytical methods and associated quality assurance procedures are included in this report in Appendix 1.

\section{A. Sampling Methods}

Methods and quality assurance procedures used to obtain soil gas samples are documented ir: Sampling Method SM1 in Appendix 1. Samples were generally obtained from depths of 36" to 48". Actual sample depths for this survey are recorded in the Soil Gas Sample Collection Log in Appendix II. Samples for the light hydrocarbon analyses were taken in previously evacuated $125 \mathrm{ml}$ bottles. Samples for volatile organic compound (VOC) aralyses were taken in previously evacuated $22 \mathrm{ml}$ bottles. A duplicate sample and a blank was collected for every ten samples. Each duplicate sample was taken from a separate location which was approximately $1 \mathrm{ft}$. from the original site. Logs which document the details of sample collection are included in Appendix II. Soil samples for mercury analyses were taken from a depth of 12 to 18 inches using the methods documented in Sample Method SM4 in Appendix 1. A duplicate sample, from a separate location approximately $1 \mathrm{ft}$. away, was taken for every ten samples. Details of the collection of soil samples for mercury are contained in the Mercury Sample Collection Logs in Appendix II.

\section{B. Analytical Methods}

Methods and quality assurance procedures for the analysis of light hydrocarbon soil gas samples are documented in Analytical Method AM1 and for VOC soil gas samples in Analytical Method AM4 in Appendix I. Methods AM1 and AM4 are modifications of EPA Headspace Method 5020. Representative chromatograms of a sample and a blank from this data set are shown on Figures $2 a$ and $2 b$, and Figure 3 . Methods and quality assurance procedures for 
the analysis of mercury in soils are documented in Analytical Method AM8 in Appendix I.

\section{Accuracy}

Calibration of the $\mathrm{C}_{\mathrm{l}} \mathrm{C}_{4}$ hydrocarbon gas chromatograph was accomplished using a certified commercial standard (Matheson Gas Products Inc.) which is of the order $10 \mathrm{ppm}$ for methane and $1 \mathrm{ppm}$ for the other light hydrocarbons and is called Standard "M". A second commercial standard (Scotty Specialty Gases, Can Mix 224, ICN \#35) was used which contains only the $\mathrm{C}_{1}-\mathrm{C}_{4}$ straight chain hydrocarbons and is of the order $1000 \mathrm{ppmv}$ for each hydrocarbon. Chromatograms of Standards " $\mathrm{M}$ " and "224" are shown on Figure 4.

Calibration of the gas chromatograph used to analyze volatila crginics was accomplished using standards prepared as a blend of pure cimpounds according to the methods documented in Standard Preparation Method SP3 which is included in Appendix I. These standards were prepared in methanol (Aldrich, HPLC grade. ICN \#30), at six concentration levels as shown on Table 4. Initially three standards from each of levels 1 through 6 were run to set up the calibration table. Thereafter, one standard was run for every 10 samples. A chromatogram of Standard "I", Level 3 for the FID is shown on Figure 5 and Level 4 for the ECD on Figure 6. Calibration of the gas chromatograph for vinyl chloride was achieved using a commercial standard gas (Scotty Specialty Gases, Can Mix 74, ICN \# 9) @ 1000 ppmv.

\section{Reliability of Selected Data}

The reliability of results presented in this report for vinyl chloride and methylene chloride is seriously affected by factors beyond experimental control; namely, in the case of vinyl chloride, the presence of two frequently occurring compounds which elute 0.07 minutes before and 0.15 minutes after the vinyl chloride elution time. The earlier peak is probably $n$-butane and the later peak is not identified, however it may be a "freon" thought to have been in the air in the on-site laboratory. For methylene chloride, an impurity thought to be derived from the 
septum, elutes 0.07 minutes before the methylene chloride elution time. Under normal laboratory conditions, we can distinguish these impurities from vinyl chloride and methylene chloride. However, during the period of these analyses, we occupied a laboratory in which temperature varied by as much as 11 degrees Fahrenheit during some 24 hour periods, causing the chromatographic retention times to vary as much as 0.7 minutes in a 24 hour period as shown for benzene in Figure 7. Under these circumstances it was not possible to reliably distinguish the desired signals from those of interfering compounds which elute very closely in time, even though retention times are adjusted to compensate for the drift due to temperature change. Thus the peak areas measured are not unequivocally attributable to vinyl chloride or methylene chloride since, if nu signal due to these compounds is present, the signal due to the nearby interfering compounds may be within the retention time window and thus be selected. As a result, we have noted on Table 2 where the measured concentration at the retention time of vinyl chloride is less than $1 \mathrm{ppm}$. in cases where no value is given, the measured concentration was larger than $1 \mathrm{ppm}$, however we have not listed these values since we believe that they may result from the above mentioned interferences, not vinyl chloride. It is at these locations that future investigations would be most likely to encounter vinyl chloride if it exists in the landfill and if confirmation of its presence is necessary.

For methylene chloride, the interference is derived largely from the septum and we have estimated the magnitude of the interference from the system blanks to be approximately $1 \mathrm{ppm}\left(\mathrm{X}_{\mathrm{avg}}+2 \sigma=0.7 \mathrm{ppm}\right)$. We have reported values for methylene chloride in Table 2 which are above $1 \mathrm{ppm}$.

\section{E. Precision}

The analytical precision was calculated using the repetitive analysis of standards as shown in Tables 5, 6, 7 and 8 . In order to derive a meaningful estimate of precision for the VOC's, the calculation was made over all standards in a data directory rather than a single survey. A data directory includes data from the analyses of all samples, standards and blanks for a period of 3 to 6 weeks. The 740-G Sanitary Landfill data reside in data directories W2, W3, and 
W9. Standards, which were run with the VOC samples and blanks from the Sanitary Landfill survey are designated on Tables 5, 6, and 7 as area "740-G".

For the VOC standards in general, percent standard deviations are less than $10 \%$. At the 10 and $100 \mathrm{ppbv}$ level, percent standard deviations for some chlorinated hydrocarbon standard analyses increase as backgrounci concentrations become a significant part of the overall signal, reaching about $20 \%$ for chloroform and 1,1,1-trichloroethane. Higher percent standard deviations for methylene chloride at the $1 \mathrm{ppmv}$ level are due to an interfering compound from the septum as discussed above in section $D$.

For the light hydrocarbons, the precision is better than $5.5 \%$ for all saturate hydrocarbons for analyses of standards " $M$ " and "224". For the unsaturates, ethylene and propylene, the precision is better than $20 \%$.

\section{F. Minimum Detection Levils}

Minimum detection levels reported in this survey for the light hydrocarbons, $\mathrm{C}_{1}$ $\mathrm{C}_{4}$, are $5 \mathrm{ppb}$. In practice a $5 \mathrm{ppb}$ standard (Microseeps Standard M diluted $200: 1)$ is reliably determined with a signal to noise ratio greater than $2(S / N>2)$. A chromatogram of the diluted standard is shown on Figure 8.

For the VOC standard, where peaks are well separated and the baseline is stable, signals on the order of 8 uv may be detected with a $S / N>4$. In a complex mixture of VOC's with many compounds, and overlapping peaks, we have determined that signals of the order 20 uv are the smallest that can be reliably and routinely determined. This corresponds to a concentration of about $70 \mathrm{ppbv}$ for the gasoline range compounds; 5 ppbv for the tri- and tetrahalocarbons; and $20 \mathrm{ppb}$ for trans 1,2-dichloroethylene. We have visually checked the integration of each peak for these compounds down to these concentration levels. We have not reported concentration levels for smaller signals, though in some individual cases they may be valid. 
Under ideal corditions where an $8 \mu \mathrm{v}$ signal can be reliably integrated, minimum detection levels may be lower. Experience has shown that with our system, an $8 \mu \mathrm{v}$ signal corresponds to approximaiely 40 area counts. We have used our calibration factors to calculate the amount of each compound corresponding to 40 area counts as shown on Table 9. In practice, we have set the "area reject" of our system to 40 area counts, which rejects lesser areas and minimizes the integration of noise related events.

\section{G. Blankis}

System blanks are collected after normal purging of the probe sampler at each 10th sample location. Ambient air is drawn through the probe then displaced into evacuated vials. The vials are then given the same consideration as samples and analyzed as such. The purpose of the system blanks are to determine if and when sample to sample contamination occurs, and also to help estainlish background levels throughout the sampling/analysis process.

Results of the analyses of all Landfill blank samples are shown on Table 10. The symbol "ND" means "not detected" and suggests an area of less than 40 area counts as discussed above. For the calculation of means and standard deviations, the amounts indicated on Table 9 for 40 area counts were used when "ND" is indicated. Results of the analyses of blank samples for the 740-G, Sanitary Landfill data set are also included on Tables 1 - 3, and a representative chromatogram is shown on Figure 3. Amounts measured in the blanks have not been subtracted from the reported sample concentrations in this report.

\section{H. Data On Magnetic Disk}

All analytical data, including samples, duplicates, standards, and blanks are provided (one copy) on magnetic disk. All data are in units of ppmv or ppbv as reported on Tables 1 - 3. 
All Jata are in Lotus 123 (Release 2, 1985) spreadsheet format using DOS (Version 5.0). VOC analytical data are accumulated and processed via Microseeps gas chromatographic data system.

\section{RESULTS}

The results of the soil gas survey at the Sanitary Landfill are recorded in Tables $1-3$, and selected data are mapped on Figures 9 - 79. In general, levels of all volatile organics monitored in this survey are above background in specific areas of the landfill. With the exception of methane, which was largely generated in the Landfill, levels and distribution of species detected reflect burial of materials which contained these species.

\section{A. Methane}

Levels of methane encountered in soil gases at the Sanitary Landfill are recorded in Table 3 as both percent methane and parts per million by volume. The distribution of methane is shown on Figures $9-11$ in terms of percent methane. The overall distribution is best understood from the symbol map shown on Figure 9 , in which the symbol size is proportional to the square root of the percentage methane.

Methane is the product of biological oxidation of cellulose and other organic substrates followed by biological reduction of the produced carbon dioxide. It is clear that methane concentrations are much more developed in the northern area of the landfill than the southern area. In the northern area, levels are consistently high to the west of the access road than they are to the east. It is not known whether this distribution is reflective of a difference in relative amounts of biologically degradable material in these different parts of the Landfill, or simply to differences in the age of burial. It has been stated that the north area was closed in 1978, however no indication was given of the order of use between the western and eastern portions of this area. Burial in the southern area is more recent. Trenches on the northern edge of the southern area were being 
filled while sampling was being carried out for this survey. Levels of methane in the south area are much lower, although two sites were found to have levels of $14 \%$ and $26 \%$. The first of these sites was associated with burial of carcasses,.

No evidence of methane inigration from the Landfill was found in the boundary sites outside the area of active burial. Methane levels in the "background area" to the north were considered background. This area is currently being developed as an extension of the landfill for active burial.

\section{B. The Saturate Hydrocarbons, $\mathrm{C}_{5}-\mathrm{C}_{10}$}

Coricentrations of the saturate hydrocarbons, propane through decane, are shown in Tables 1 and 3 and the distribution of these species are shown on Figures $12-38$. All of these species may be components of gasoline, although propane would certainly be a minor constituent of this fluid. Propane and the butanes have been usea as propellants for certain aerosols, while pentane and the higher hydrocarbons may be a part of many petroleum mixtures or indeed used as pure solvents. Differences in distribution pattern between the light saturates and the heavy saturates may reflect burial 0 : materials having different compositional characteristics. Any observed distribution, however, may have been altered since the time of burial since all of these species readily undergo biological oxidation in the presence of bacteria and sufficient oxygen. In general, rates of oxidation decrease with decreasing number of carbons and straight chain saturates are oxidized in preference to branched or cyclic saturates. In extreme cases, gasoline range saturates may be entirely oxidized to carbon dioxide and water. Some or all of the carbon dioxide produced may subsequently be reduced to methane.

\section{The Unsaturated Hydrocarbons Ethylene and Propylene}

Concentrations of ethylene and propylene are listed on Table 3, and their distributions are shown on Figures 39 - 44. 
Both species may have been buried in the Landfill as a part of the materials discarded by facilities at SRS, however both gases are found naturally as a part of uncontaminated soil gases. It is generally thought that there may be some biological mechanism for their formation, however details of their generation in this manner are not well docu,nented.

\section{The Aromatic Hydrocarbons}

Concentrations of the aromatic hydrocarbons monitored; benzene, toluene, ethylbenzene, and the xylenes, are shown on Table 1 and their distributions are shown on Figures 45 - 59. These compounds are components of many petroleum based oils, solvents, and fuels. The presence of these compounds in the soil gases at the landfill is evidence that such petroleum based materials have been a part of the materials buried.

The large benzene anomaly at site 69 in the north area is accompanied by the other aromatics and the entire suite of gasoline range saturates. This is suggestive, but not conclusive, evidence of gasoline. In general, the heavier aromatics (xylenes, ethylbenzene and toluene) are much more prevalent in the northern area than benzene. This may be attributable to the greater solubility of benzene in water than the heavier aromatics, but could also be explained by the presence of materials such as paints or oils with component distributions in which the heavier of these aromatics are more prominent.

In general, levels of the aromatics in the south area are lower than in the northern area although selected sites were found to have high concentrations, particularly of the heavier aromatics.

\section{E. The Chlorinated Hydrocarbons}

Concentrations of the chlorinated hydrocarbons monitored are shown on Table 2 , and their distribution is shown on Figures 50-77. In general, methylene chloride, chloroform and trans 1,2 dichloroethylene are minor constituents of the soil air at the Landfill relative to 1,1,1-trichloroethane, trichloroethylene, and 
tetrachloroethylene. No carbon tetrachloride was observed above its minimum detection level of $5 \mathrm{ppbv}$. Tetrachloroethylene was found at $140 \mathrm{ppm}$ at site 34 in the northern area and was present at lower levels at many sites in both the northern and southern areas. A larger amount of 1,1,1-trichloroethane was found in the southern area than the northern area. Trichloroethylene was found in many sites in both the northern and southern areas.

\section{F. Mercury}

Mercury levels measured in soils at a depth of 1 foot are in general very low. The highest levels recorded were 33 and $31 \mathrm{ng} / \mathrm{g}$. While these levels are above the general background observed at the Landfill, they may represent extrema in the normal distribution rather than evidence of buried mercury. Note that one site in the "background area" was observed to have $13 \mathrm{ng} / \mathrm{g}$.

\section{CONCLUSIONS}

The data presented in this report confirm that a variety of common petroleum based fluids and chlorinated solvents have been a part of the materials buried at the 740-G Sanitary Landfill. Levels of the species monitored greatly exceed background concentrations.

In an independent study of the effects of barometric pumping on soil gases at this site (WSRC-MS-92-877), it was shown that during periods of falling barometric pressure, all species found in the soil gas survey discussed in this report migrate through the soil cover into the atmosphere.

Methane levels, although high, are to be expected for a landfill where large amounts of cellulose and organics have been buried. It is known that even large organic species have a significant solubility in methane under high pressure. It may be that methane in high concentrations, even at atmospheric pressure, is a factor in the migration of the iarger organic species. 
This page intentionally left blank. 
Figures and Tables 
This page intentionally left blank. 


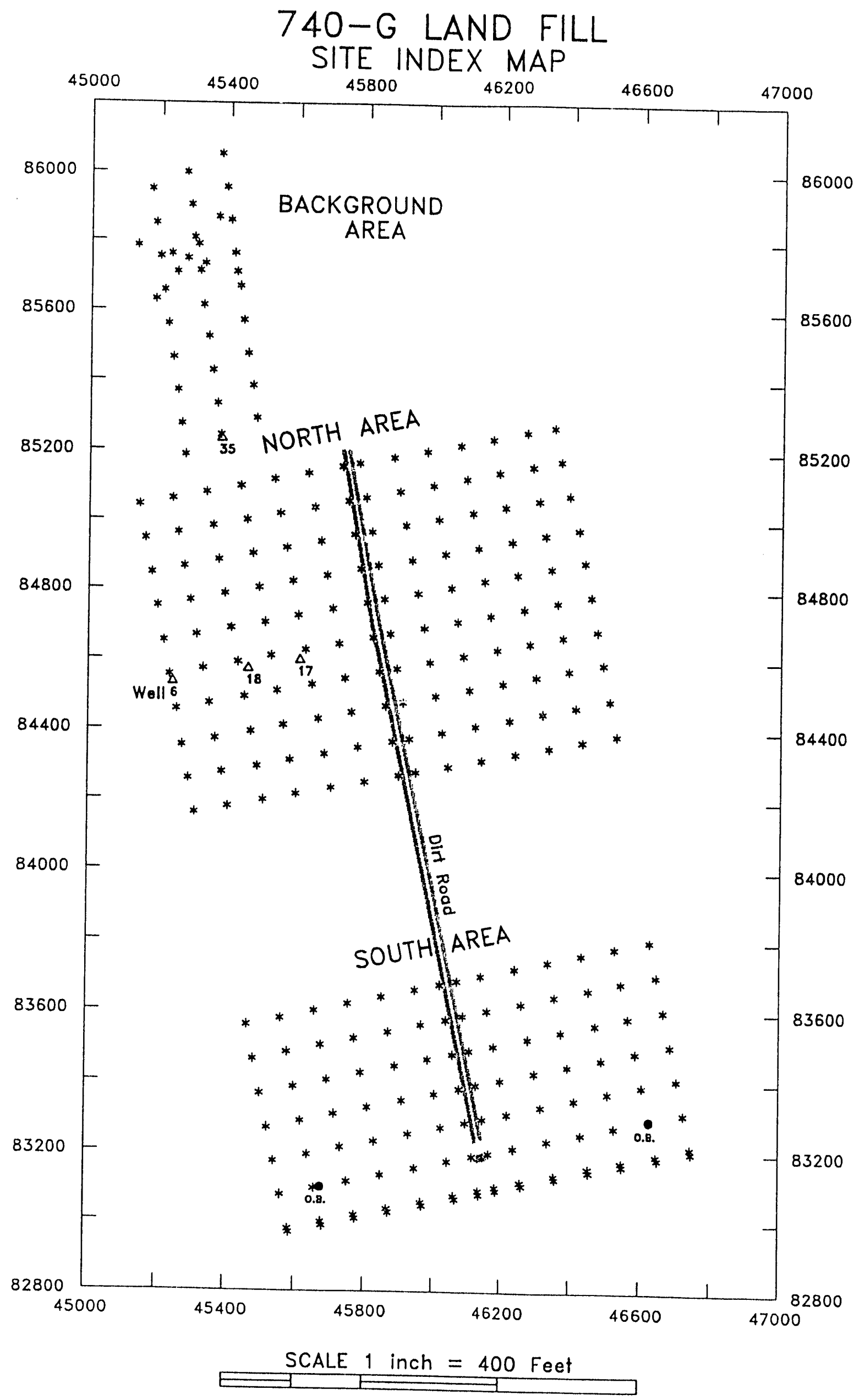

Figure 1a. The 740-G Sanitary Landfill site Index Map (all locations o $1 "=400 \mathrm{ft}$ ) 


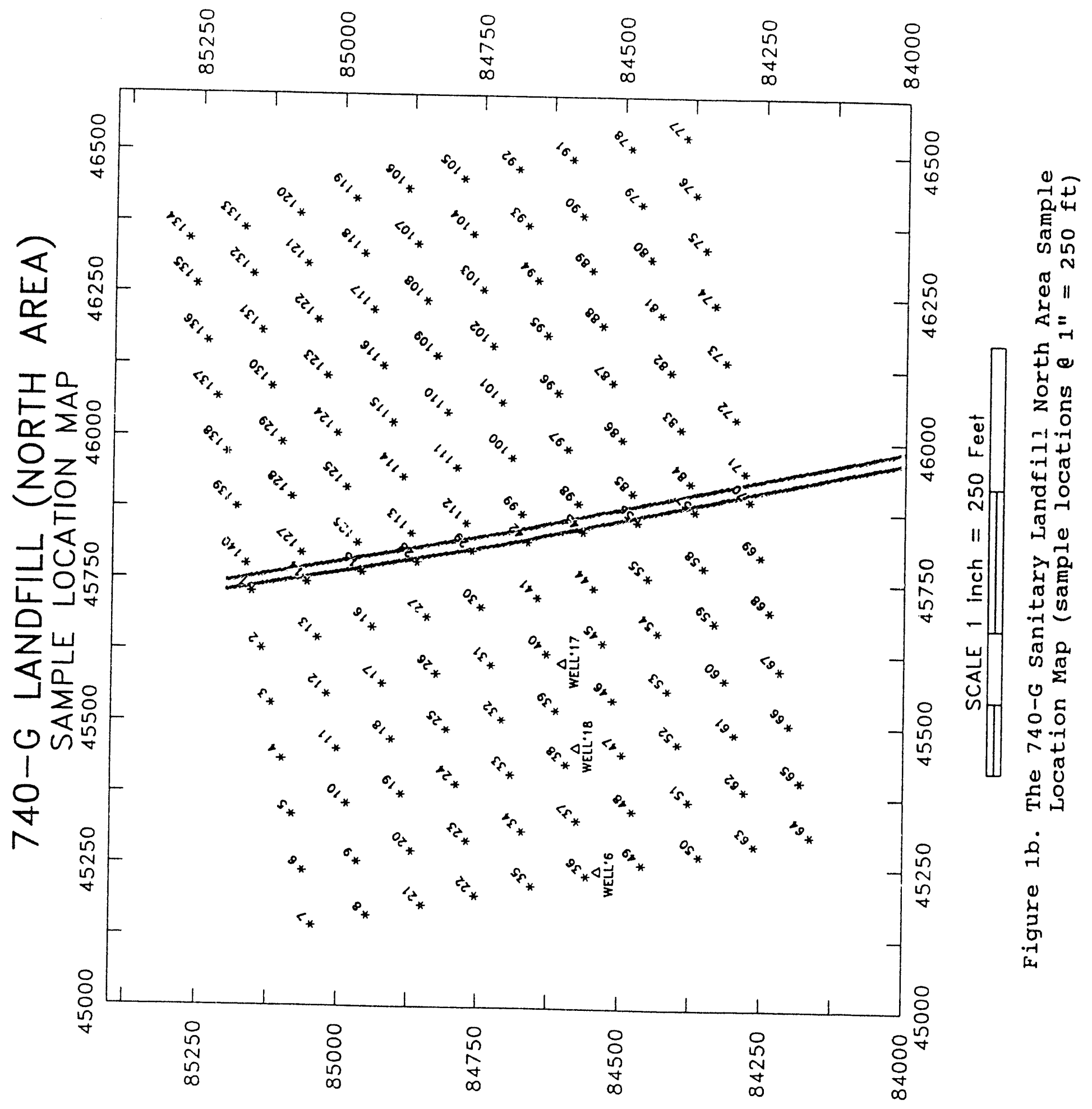




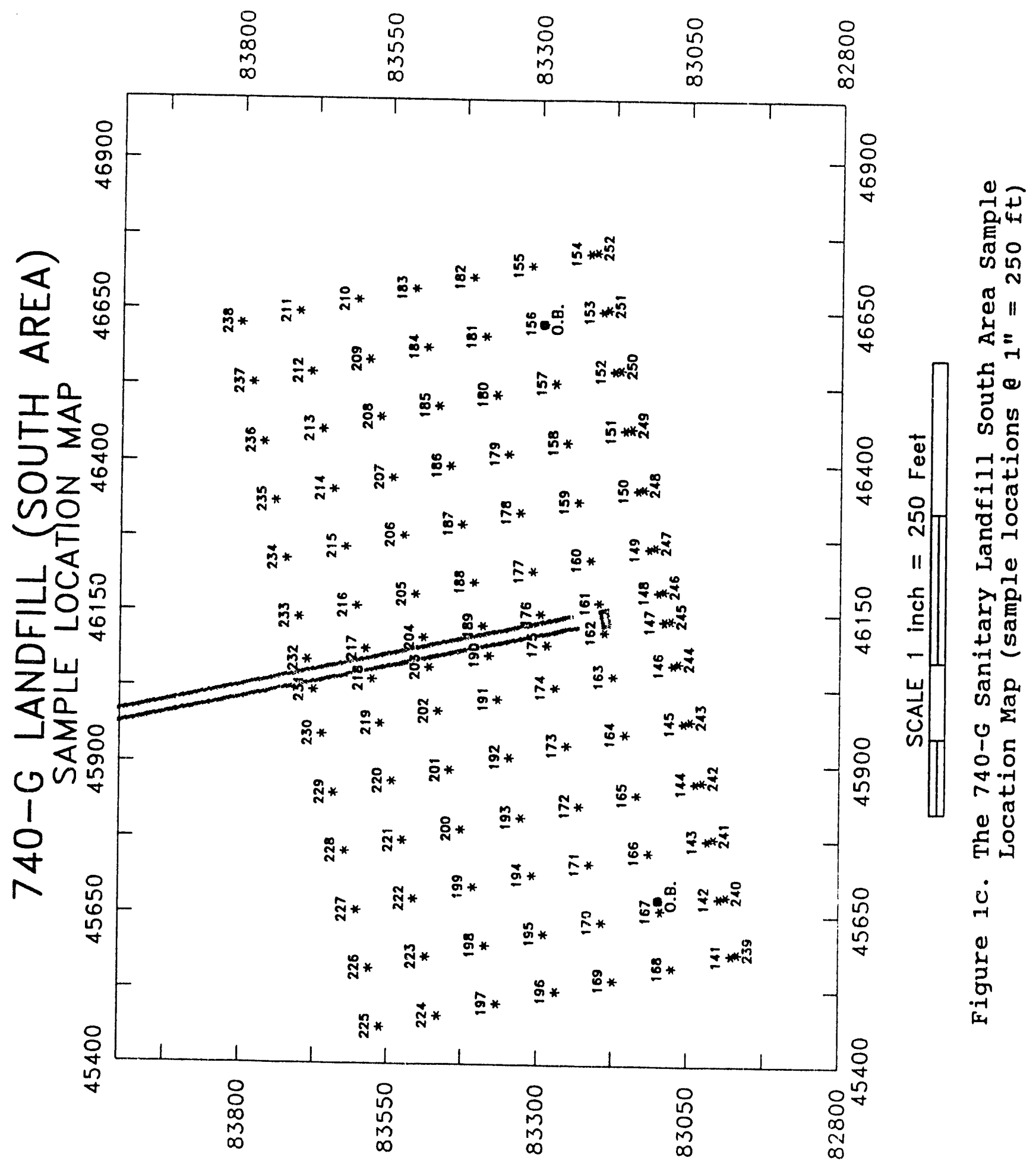




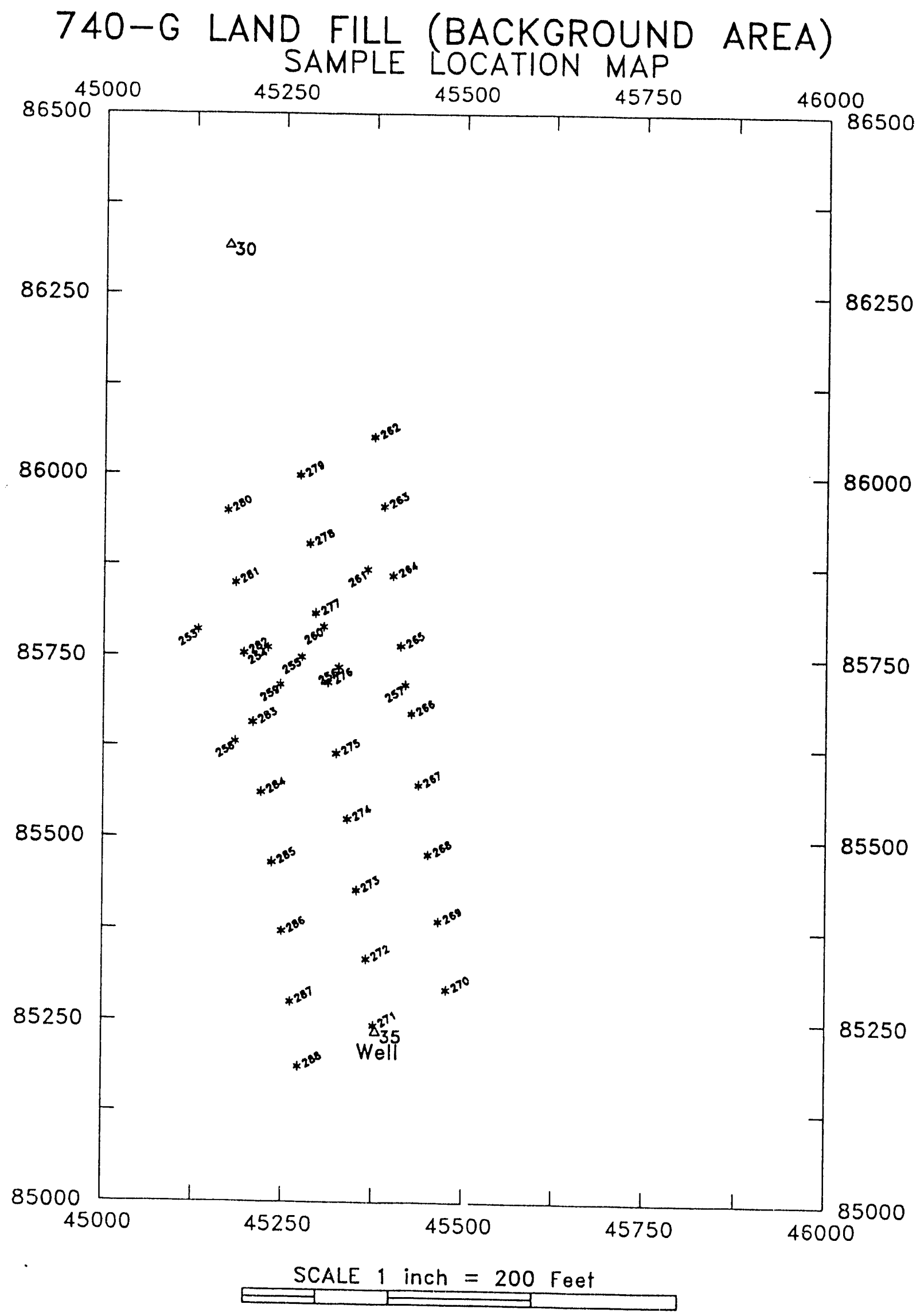

Figure 1d. The 740-G Sanitary Landfill Background Area Sample Location Map (sample locations $\mathrm{e} 1^{\prime \prime}=200 \mathrm{ft}$ ) 
File $=C:$ \CP \DATA4 $W 2 A 1.65 R$ Date printed $=11-22-1991$ Time $=16: 43: 30$

3.00 to $30.00 \mathrm{~min}$. Low $Y=-12.52761 \mathrm{mv}$ High $Y=3300.10083 \mathrm{mv} \quad$ Span $=3312.62842 \mathrm{mv}$

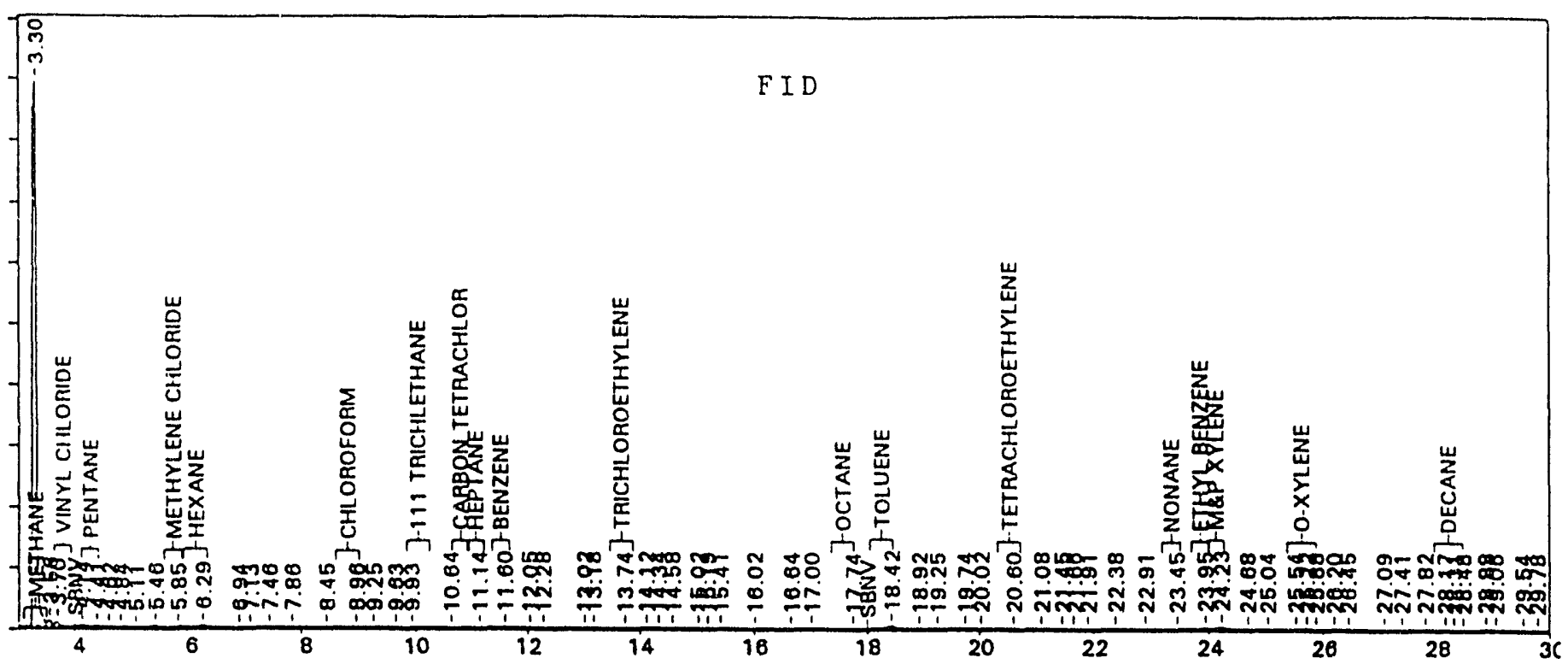

File=C: \CP \DATA4 IW2A1.65R Date printed $=11-22-1991$ Time $=16: 45: 06$

4.00 to $30.00 \mathrm{~min}$. Low $Y=-0.32111 \mathrm{mv}$ High $Y=14.08442 \mathrm{mv} \quad$ Span $=14.40552 \mathrm{mv}$

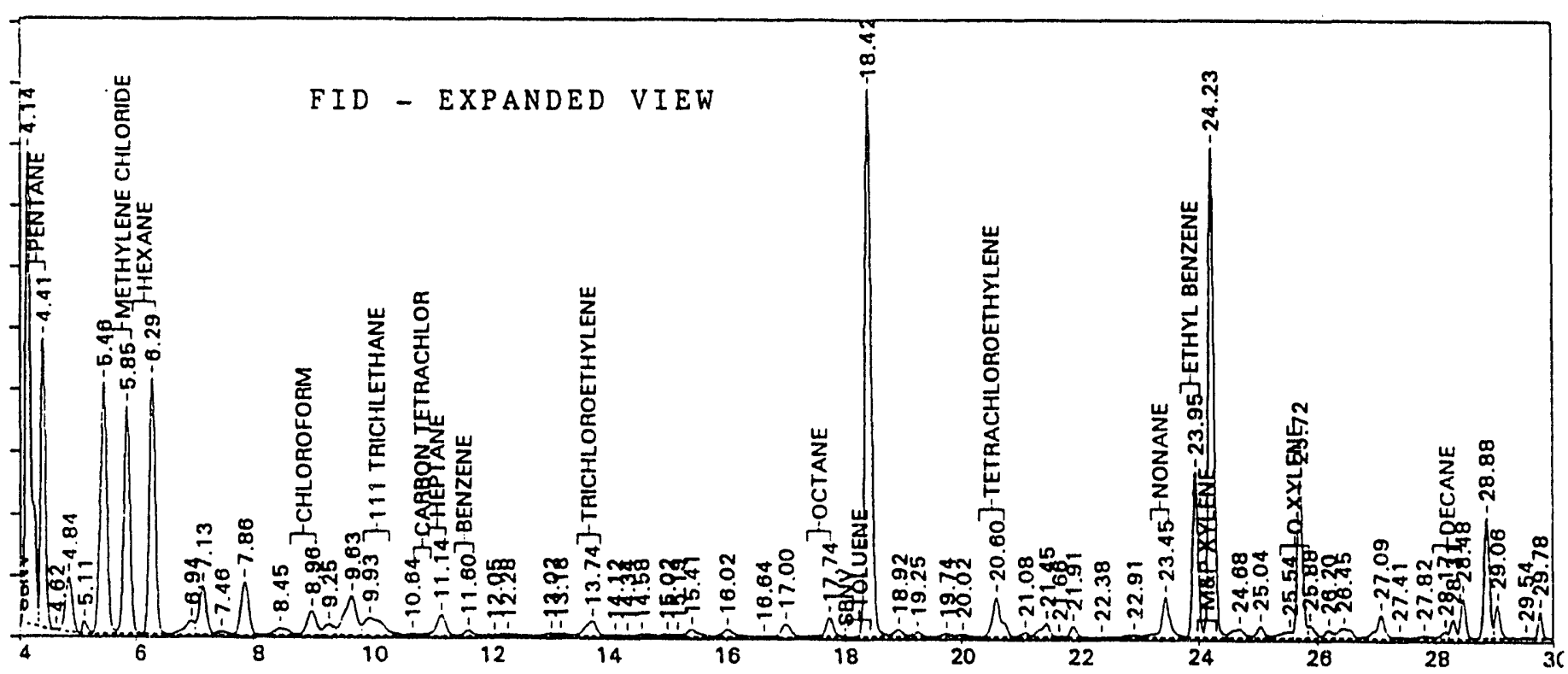

Figure 2a. Gas Chromatogram of Soil Gases at Site 111 from the Flame Ionization Detector(FID) 
File=C:ICPIDATA4IW2B1.65R Date printed $=11-22-1991$ Time $=16: 48: 46$

$-1.50212 \mathrm{mv}$

High $Y=490.56058 \mathrm{mv}$

Span $=492.06271 \mathrm{mv}$

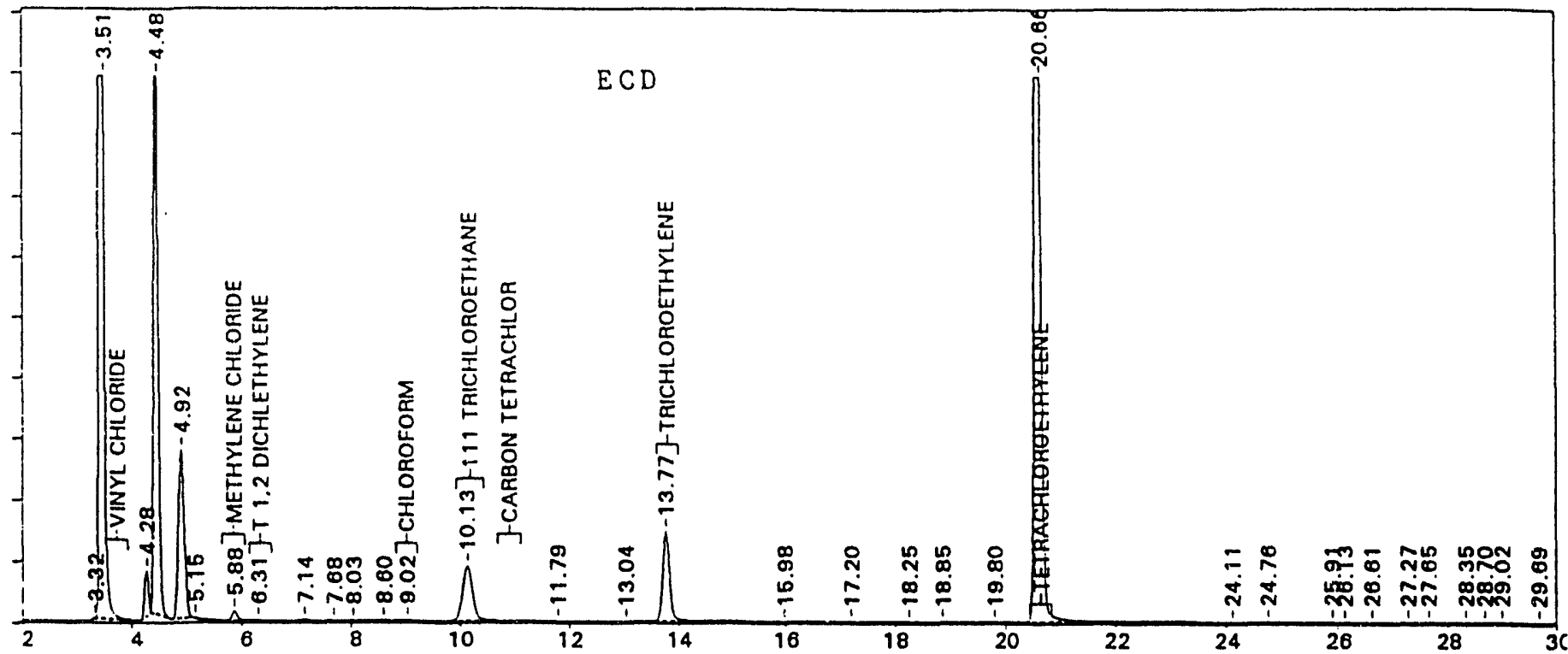

File=C:ICPIDATA4IURB1.65R Date printed $=11-22-1991$ Time $=16: 51: 39$

5.08 to $11.24 \mathrm{~min}$. Low $Y=$

$6.26096 \mathrm{mN}$

Span $=7.23551 \mathrm{mv}$

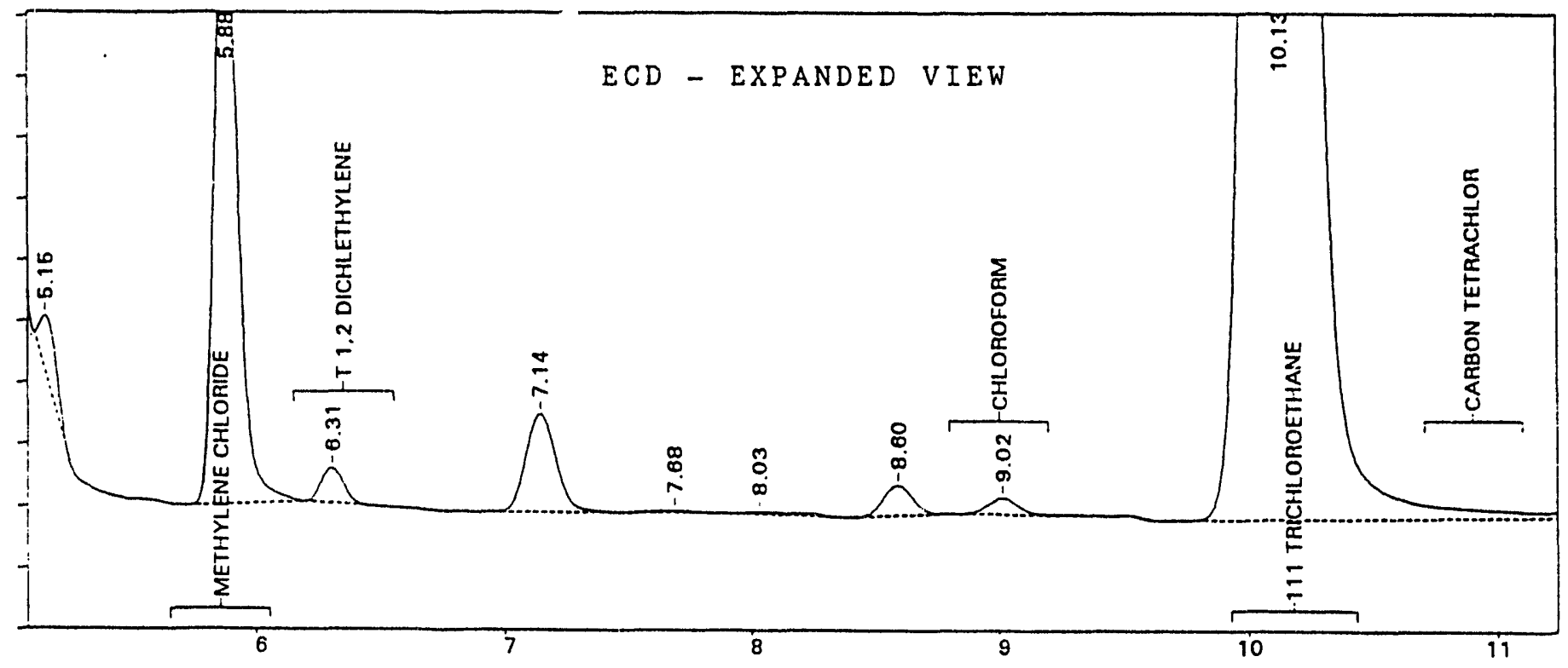

Figure 2b. Gas Chromatogram of Soil Gases at Site 111 from the Electron Capture Detector(ECD) 
File $=C:$ ICP \DATA4 \W2A.85R Date printed $=11-22-1991$ Time $=16: 57: 41$
3.00 to $30.00 \mathrm{~min}$. Low $Y=$
$-0.27952 \mathrm{mv}$
High $Y=$
$0.42976 \mathrm{mv}$
Span $=$
$0.70927 \mathrm{mv}$

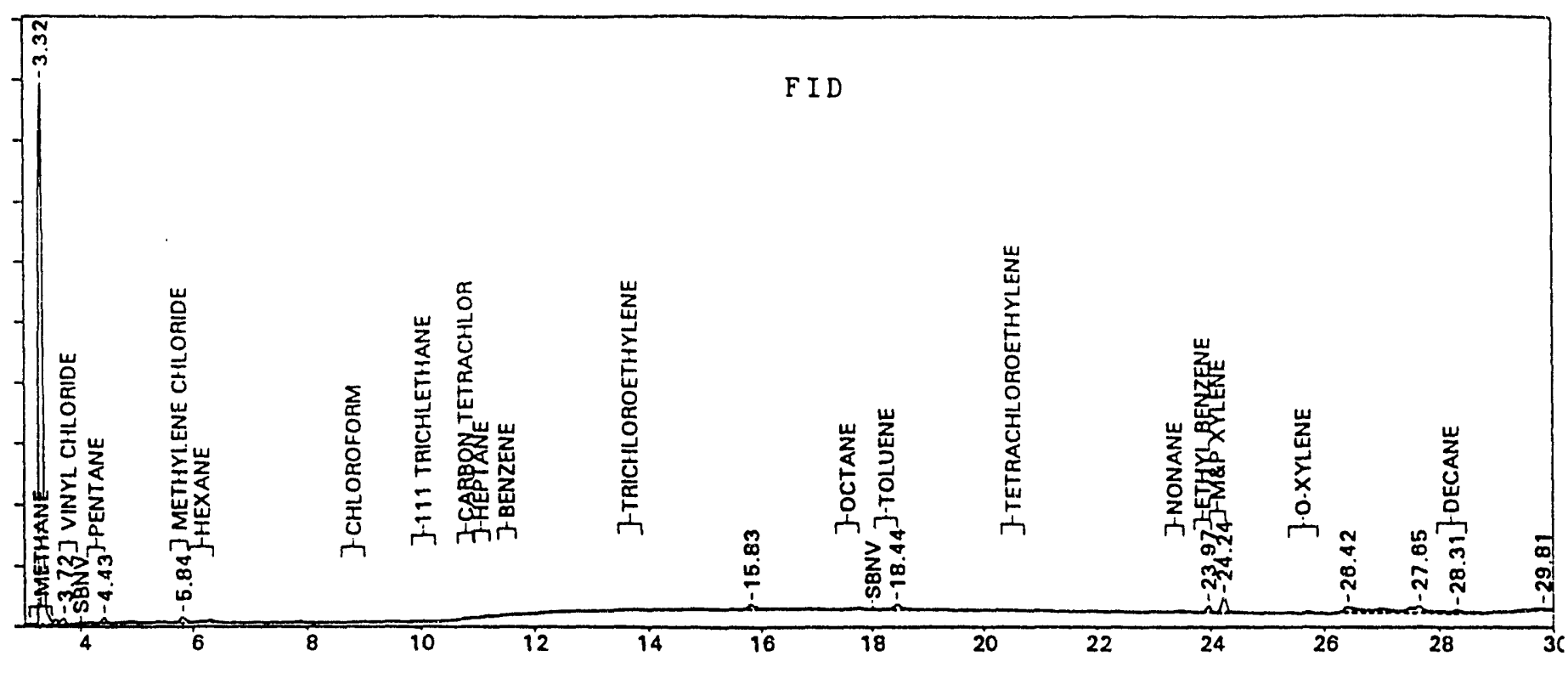

File $=$ : $:$ ICP \DATA4 $\backslash$ W2B $.85 R$ Date printed $=11-22-1991 \mathrm{Time}=16: 59: 04$

2.00 to $30.00 \mathrm{~min}$. Low $Y=0.33500 \mathrm{mv}$ High $Y=11.73912 \mathrm{mv} \quad$ Span $=11.40412 \mathrm{mv}$

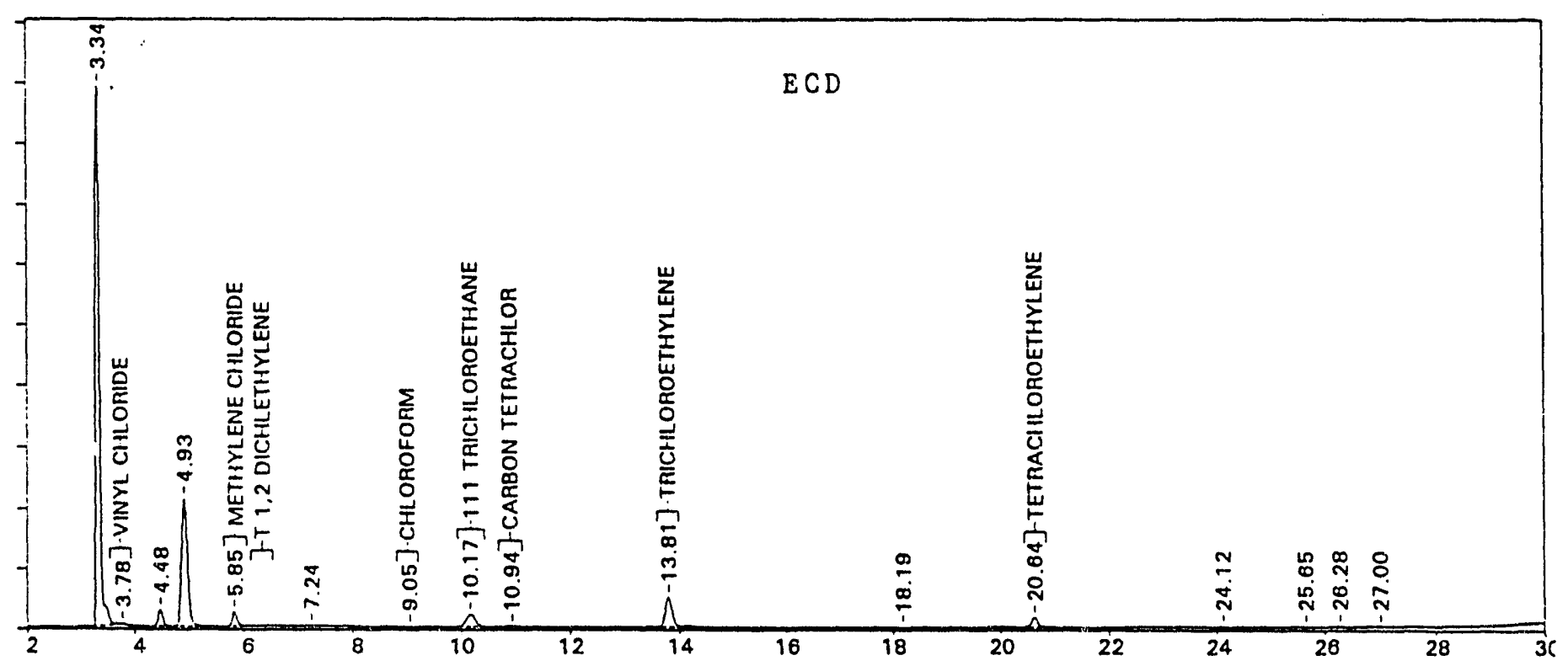

Figure 3. Gas Chromatogram of System Blank \# 7 

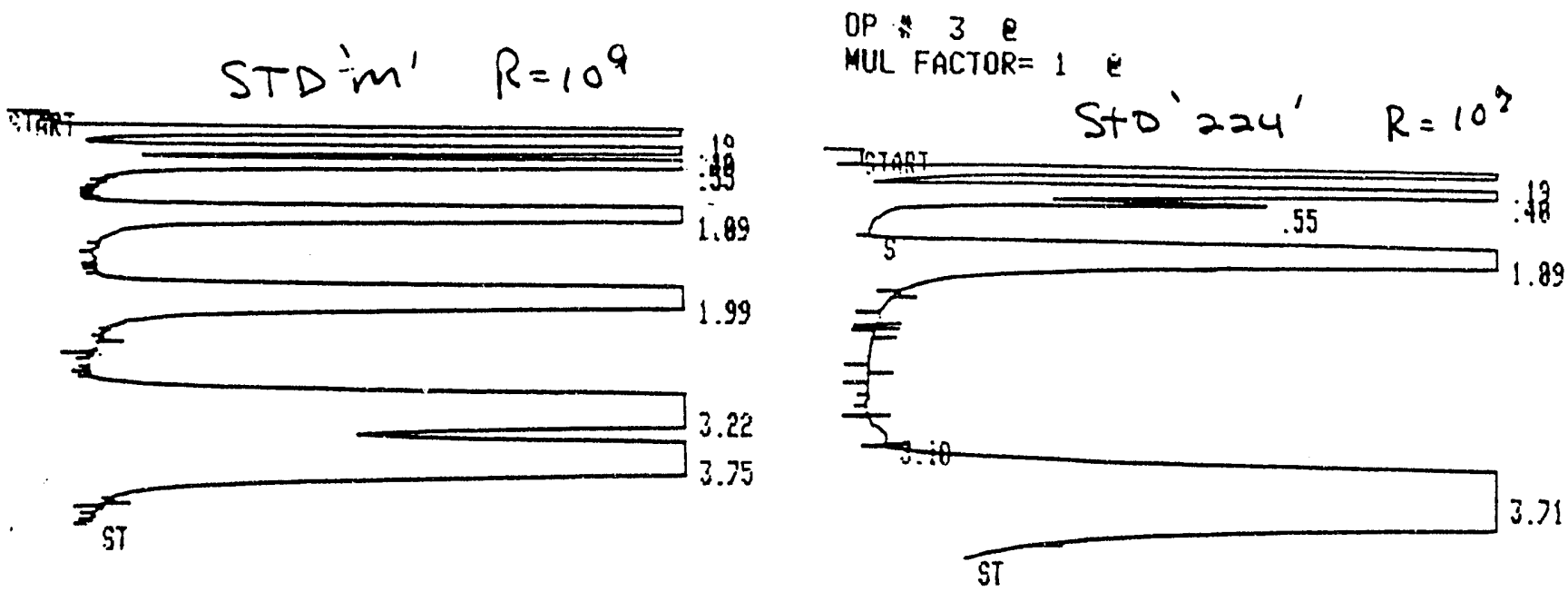

\begin{tabular}{|c|c|c|c|c|c|c|c|c|c|}
\hline \multicolumn{2}{|c|}{$\begin{array}{l}\text { RUN \# } 444 \\
\text { HORKFILE ID: B } \\
\text { HURKFILE MAHE: }\end{array}$} & \multicolumn{2}{|c|}{$\mathrm{NOY} / 13 / 90$} & $88: 59: 17$ & \multicolumn{2}{|c|}{$\begin{array}{l}\text { RUH } \$ 43 \\
\text { WORKFILE ID : } 8 \\
\text { WORKFILE NAME: }\end{array}$} & \multicolumn{2}{|c|}{ HOY $/ 13 / 90$} & \multirow[t]{2}{*}{$98: 47: 18$} \\
\hline $\begin{array}{l}\text { ESTD } \\
\text { RT }\end{array}$ & AREA & TYPE & CAL $\neq$ & AHOUNT & ESTO & & & & \\
\hline $\begin{array}{l}9.19 \\
0.45 \\
0.55 \\
1.99 \\
1.99 \\
3.22 \\
3.75\end{array}$ & $\begin{array}{r}2497368 \\
494138 \\
664670 \\
647228 \\
496458 \\
677280 \\
527898\end{array}$ & $\begin{array}{l}\text { SHE } \\
\text { TPY } \\
\text { TYY } \\
\text { TYY } \\
\text { TYY } \\
\text { PY } \\
\text { WY }\end{array}$ & $\begin{array}{l}1 \\
2 \\
3 \\
4 R \\
5 \\
6 \\
7\end{array}$ & $\begin{array}{r}10.389 \\
1.934 \\
1.938 \\
1.946 \\
1.946 \\
1.923 \\
0.997\end{array}$ & $\begin{array}{c}R T \\
Q .19 \\
5.49 \\
0.55 \\
1.99 \\
3.18 \\
3.71\end{array}$ & $\begin{array}{r}\text { ARER } \\
2961700 \\
5319790 \\
9198 \\
7429986 \\
752 \\
7548580\end{array}$ & $\begin{array}{r}\text { TYPE } \\
\text { SPH } \\
\text { SHB } \\
\text { TBE } \\
\text { BB } \\
\text { PB } \\
\text { PB }\end{array}$ & $\begin{array}{c}\text { CAL } \\
1 \\
2 \\
2 \\
3 \\
4 \\
6 \\
7 \\
7\end{array}$ & $\begin{array}{r}\text { AMOUHT } \\
1846.586 \\
1858.308 \\
1.579 \\
1620.796 \\
0.114 \\
1830.980\end{array}$ \\
\hline
\end{tabular}

Figure 4. Gas Chromatogram of $C_{1}-C_{4}$ standards "M" and " 224 " 
File=C: $\backslash$ CP \DATA4 $\backslash$ W2A3.10R Date printed $=11-22-1999$ rime $=16: 26: 49$

4.09 to $29.92 \mathrm{~min}$. Low $Y=-0.25062 \mathrm{mv}$ High $Y=6.39529 \mathrm{mv}$ Span $=6.64590 \mathrm{mv}$

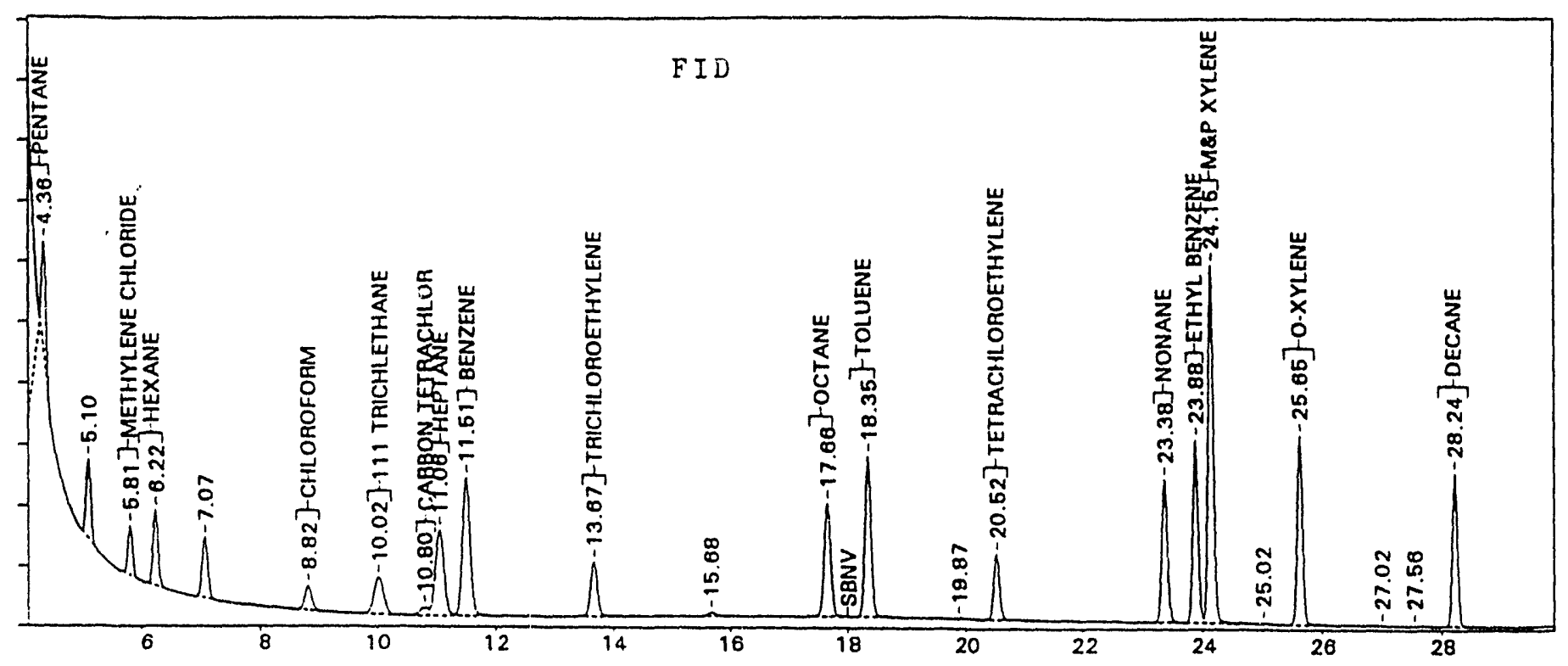

Figure 5. Gas Chromatogram (FID) of VOC Standard "I", Level 3 
File $=$ C: $\backslash$ CP \DATA4 IW2B2.98R Date printed $=11-22-1991 \mathrm{Time}=16: 35: 05$

2.00 to $30.00 \mathrm{~min}$. Low $Y=-0.05700 \mathrm{mv}$ High $Y=84.30374 \mathrm{mv}$ Span $=84.36074 \mathrm{mv}$

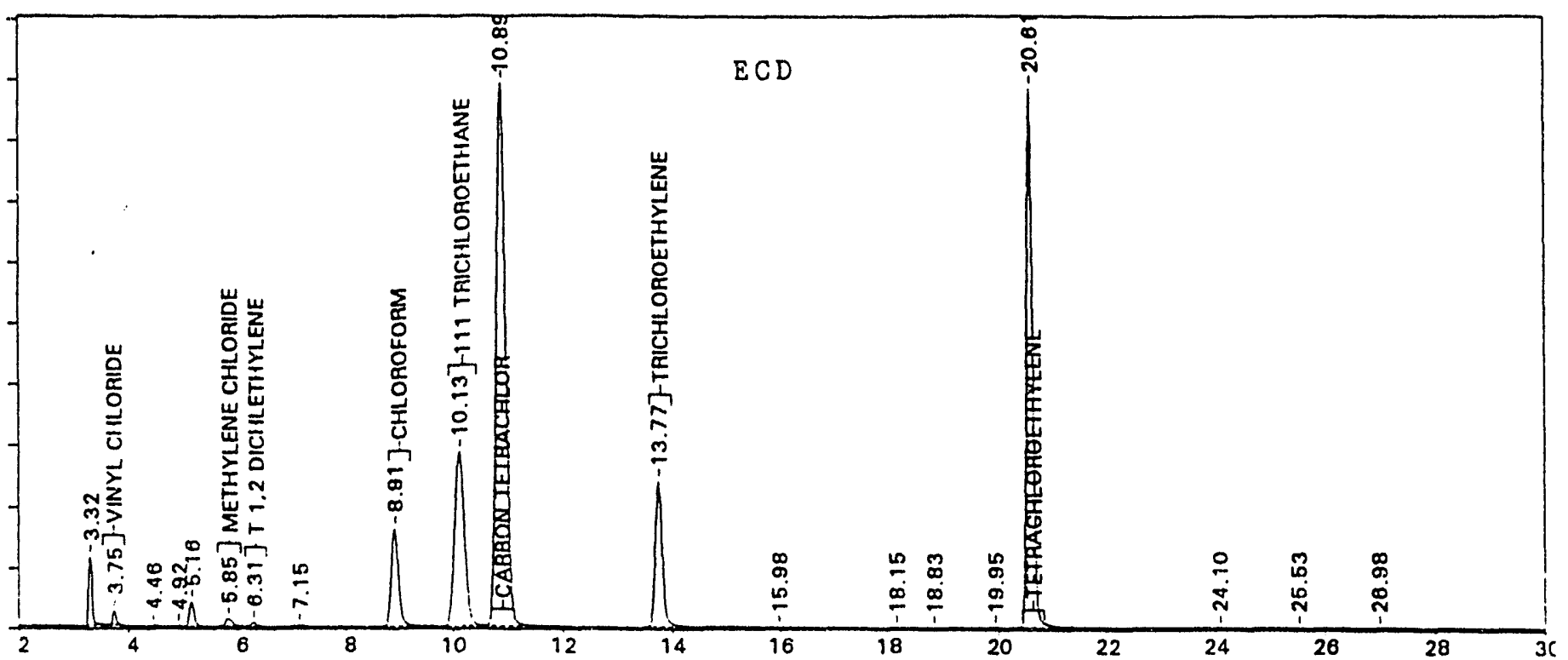

Figure 6. Gas Chromatogram (ECD) of VOC Standard "I", Level 4 
Retention Time of Benzene vs. Room Temperature

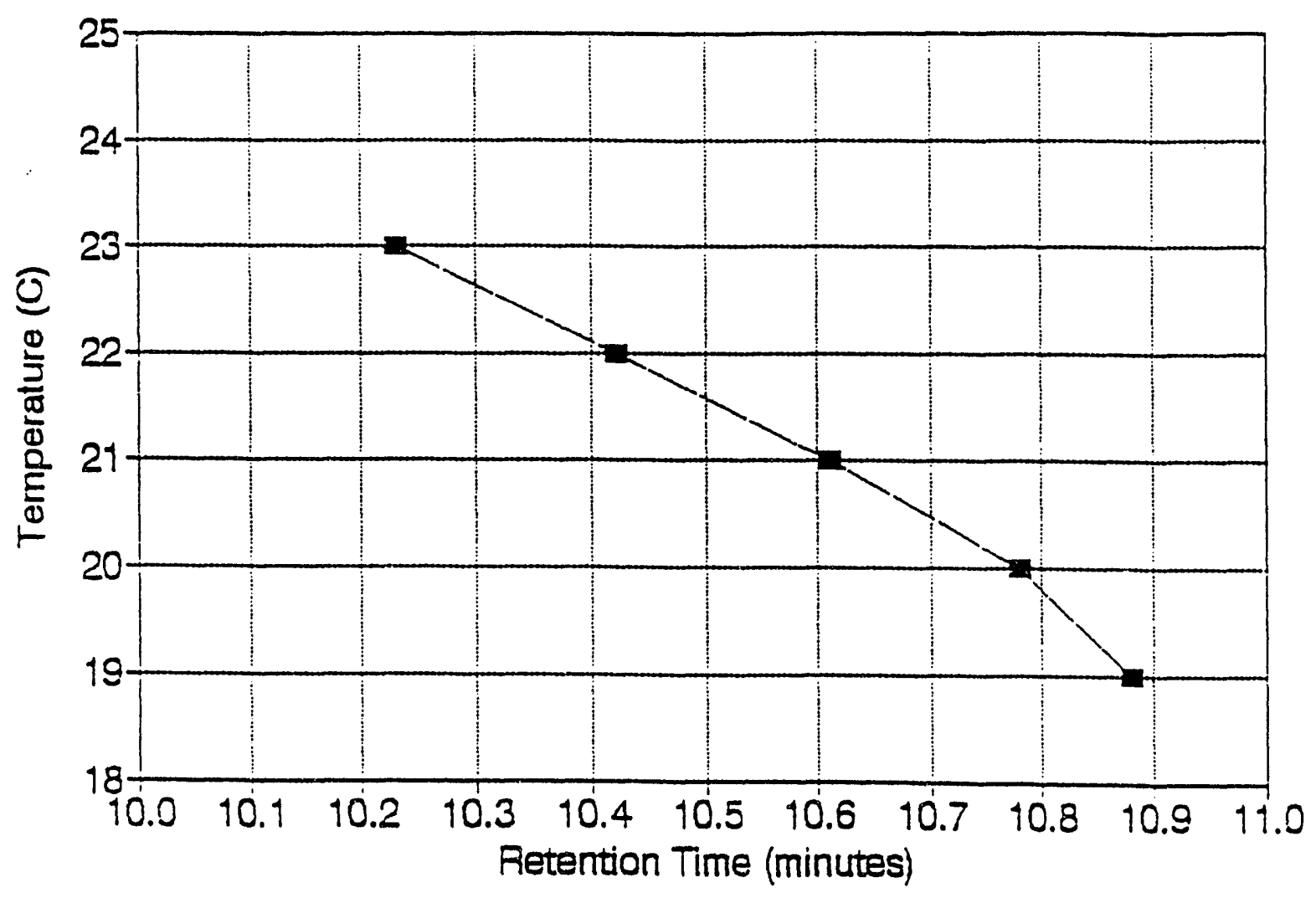

Figure 7. Benzene Retention Time vs Room Temperature 

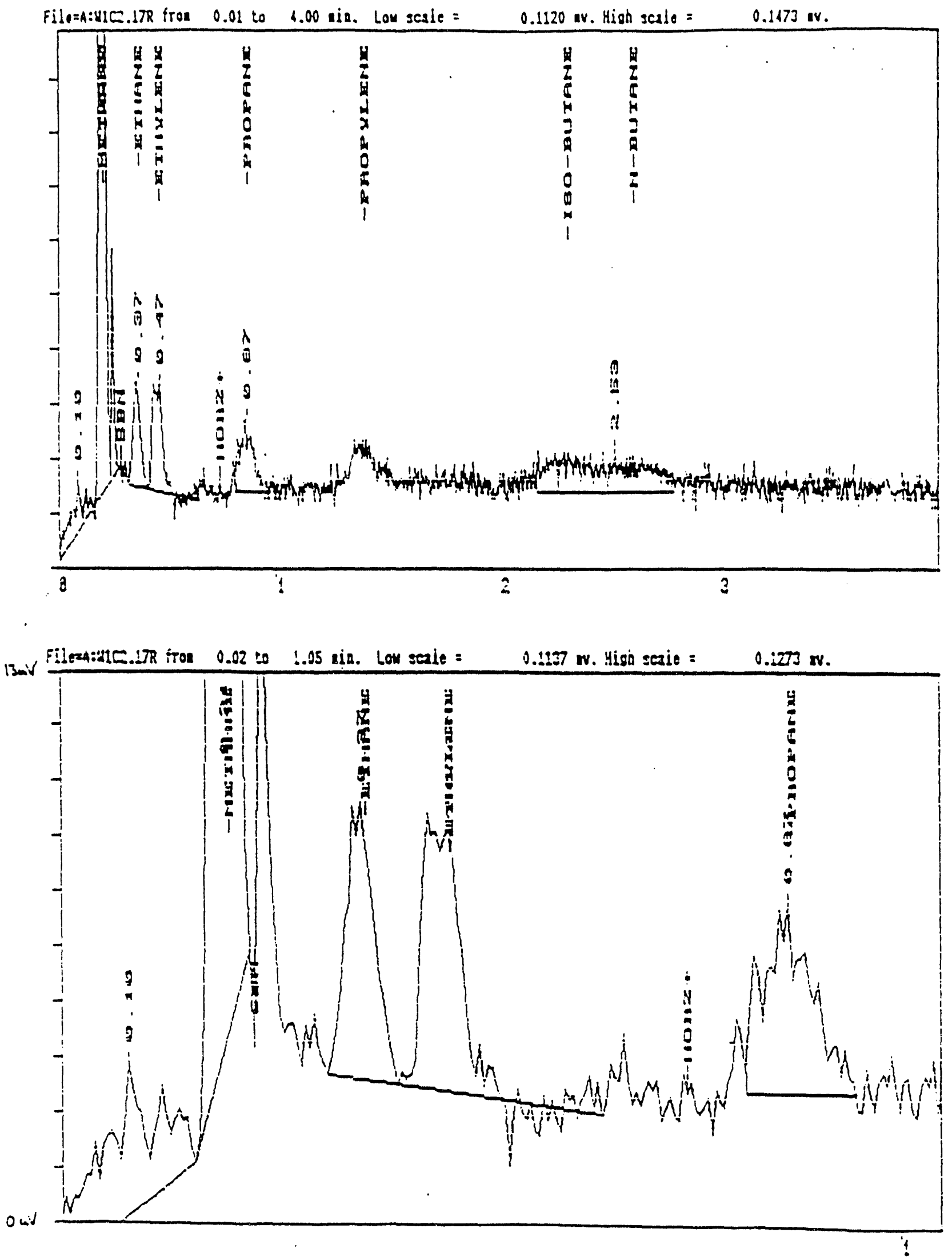

Figure 8. Gas Chromatogram of $C_{1}-C_{4}$ Hydrocarbon Standard $M$ (a) 200:1 Dilution in $\mathrm{N}_{2}$ 


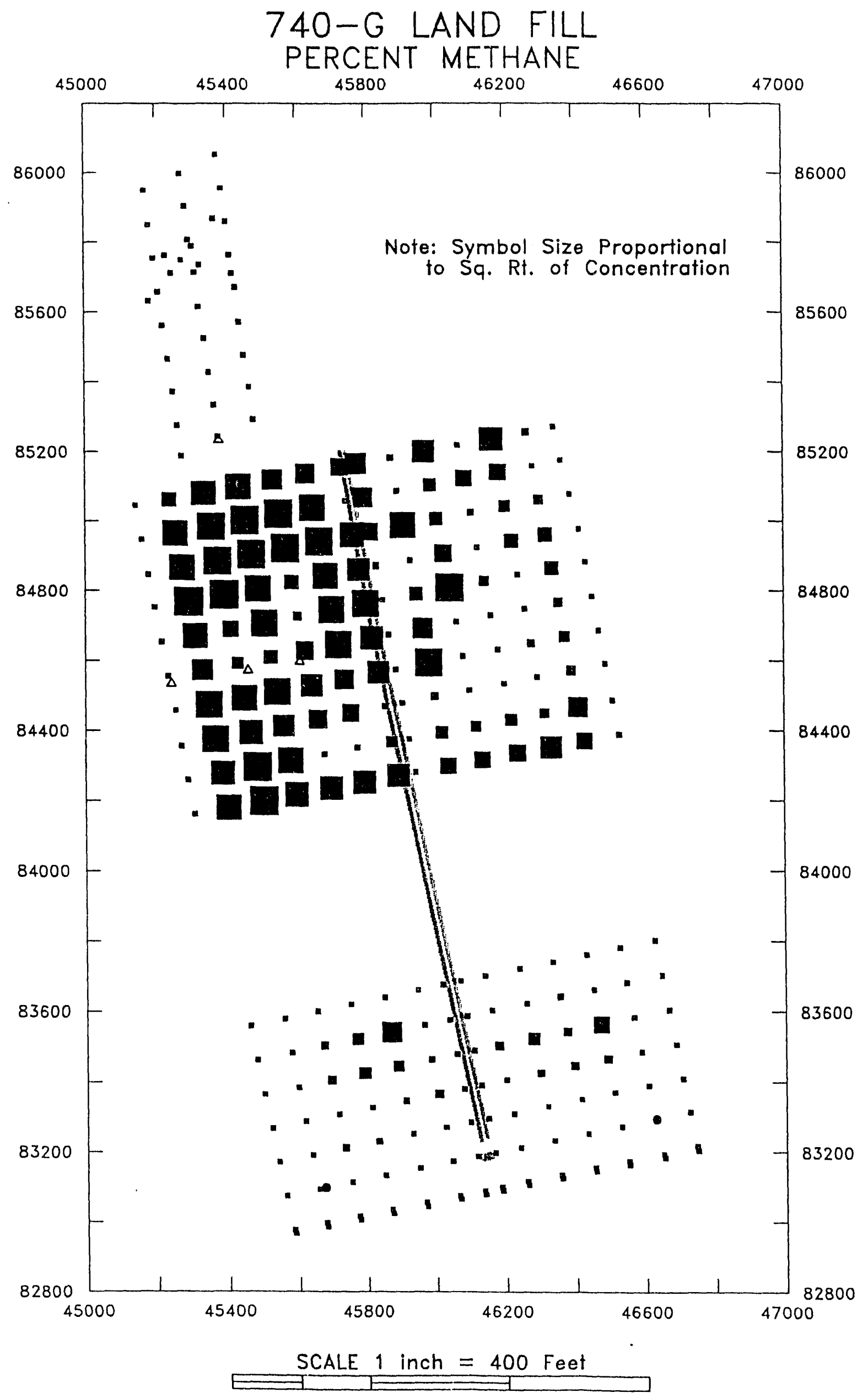

Figure 9. Methane Symbol Map 


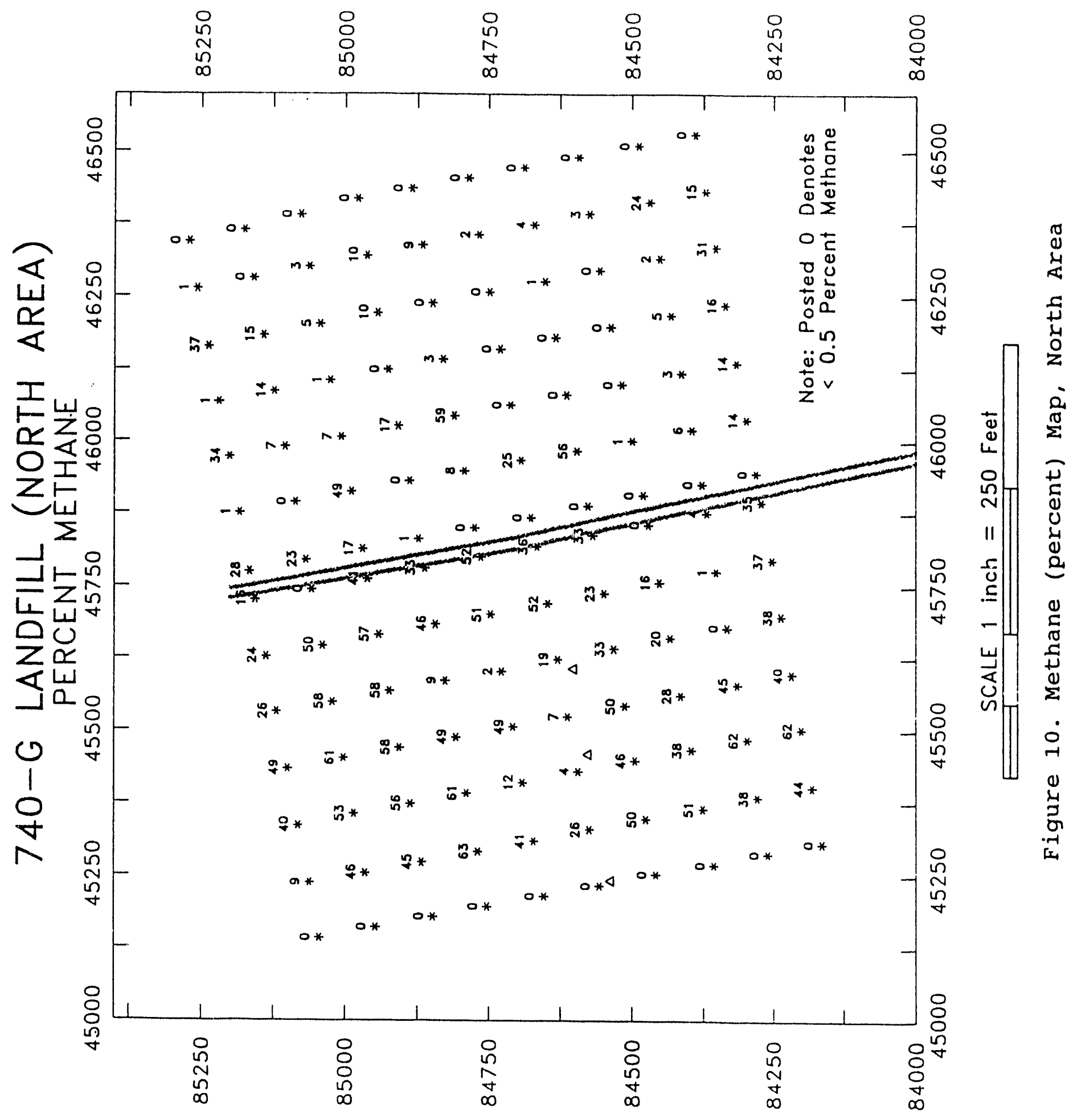




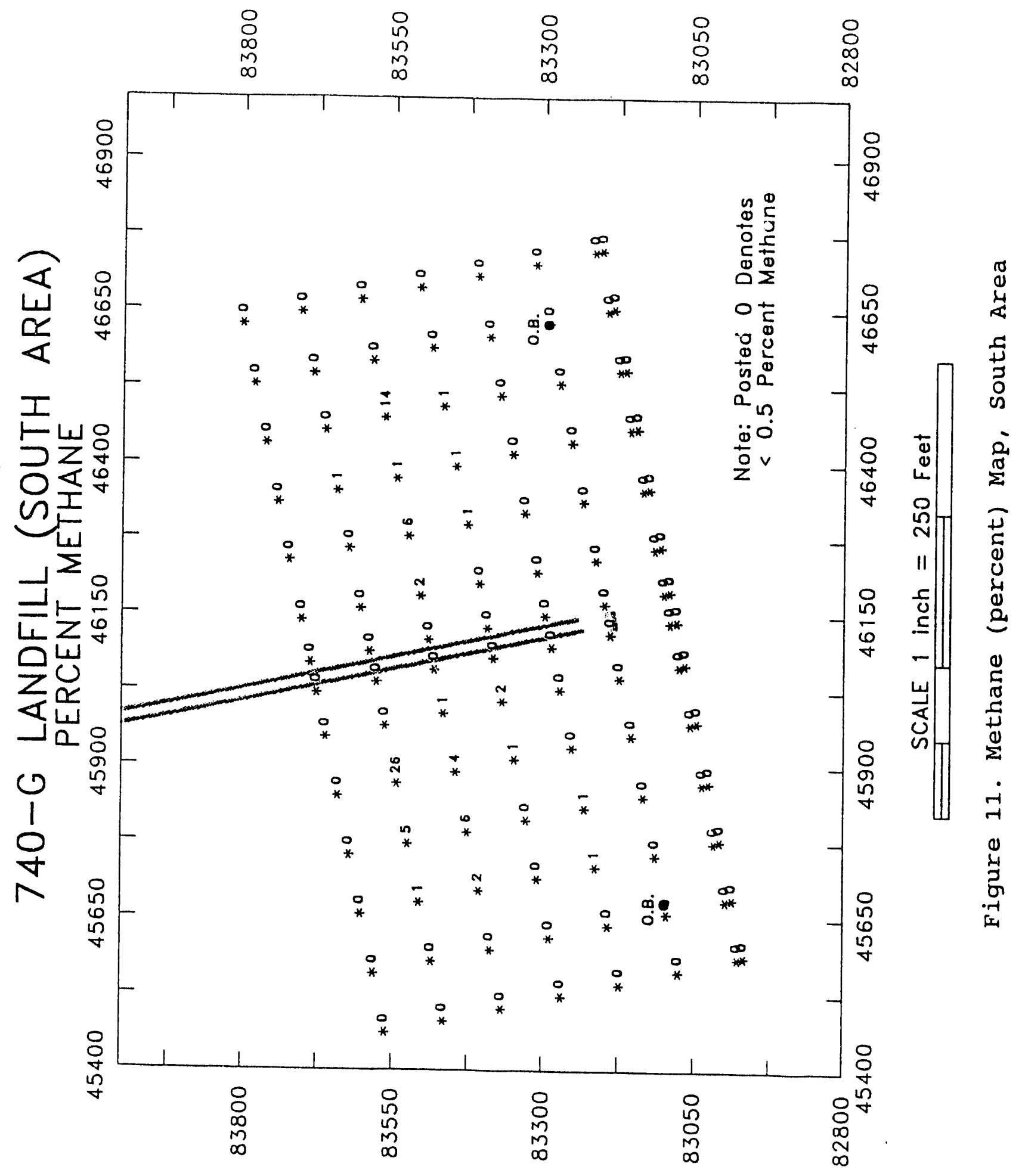




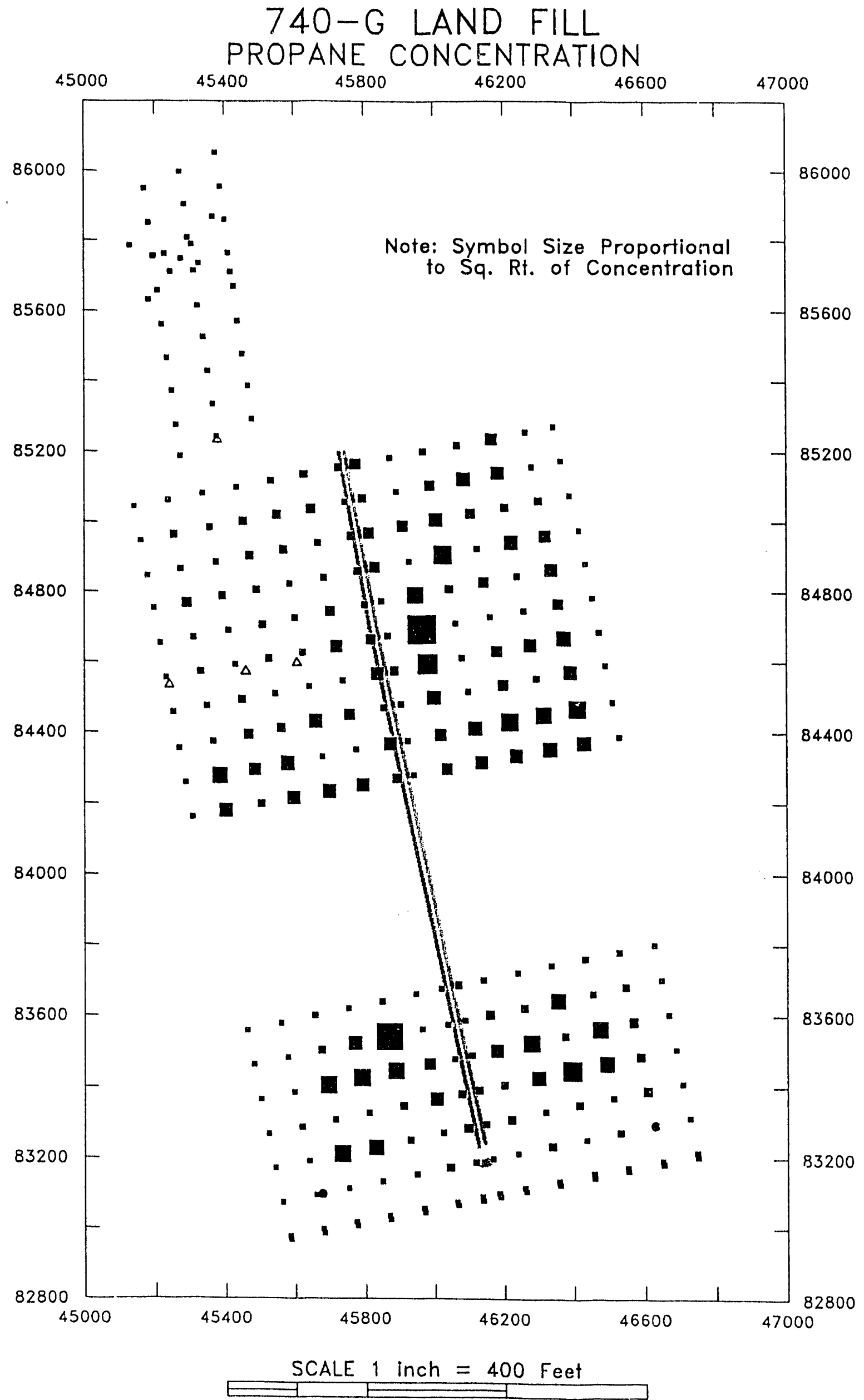

Figure 12. Propane Symbol Map 


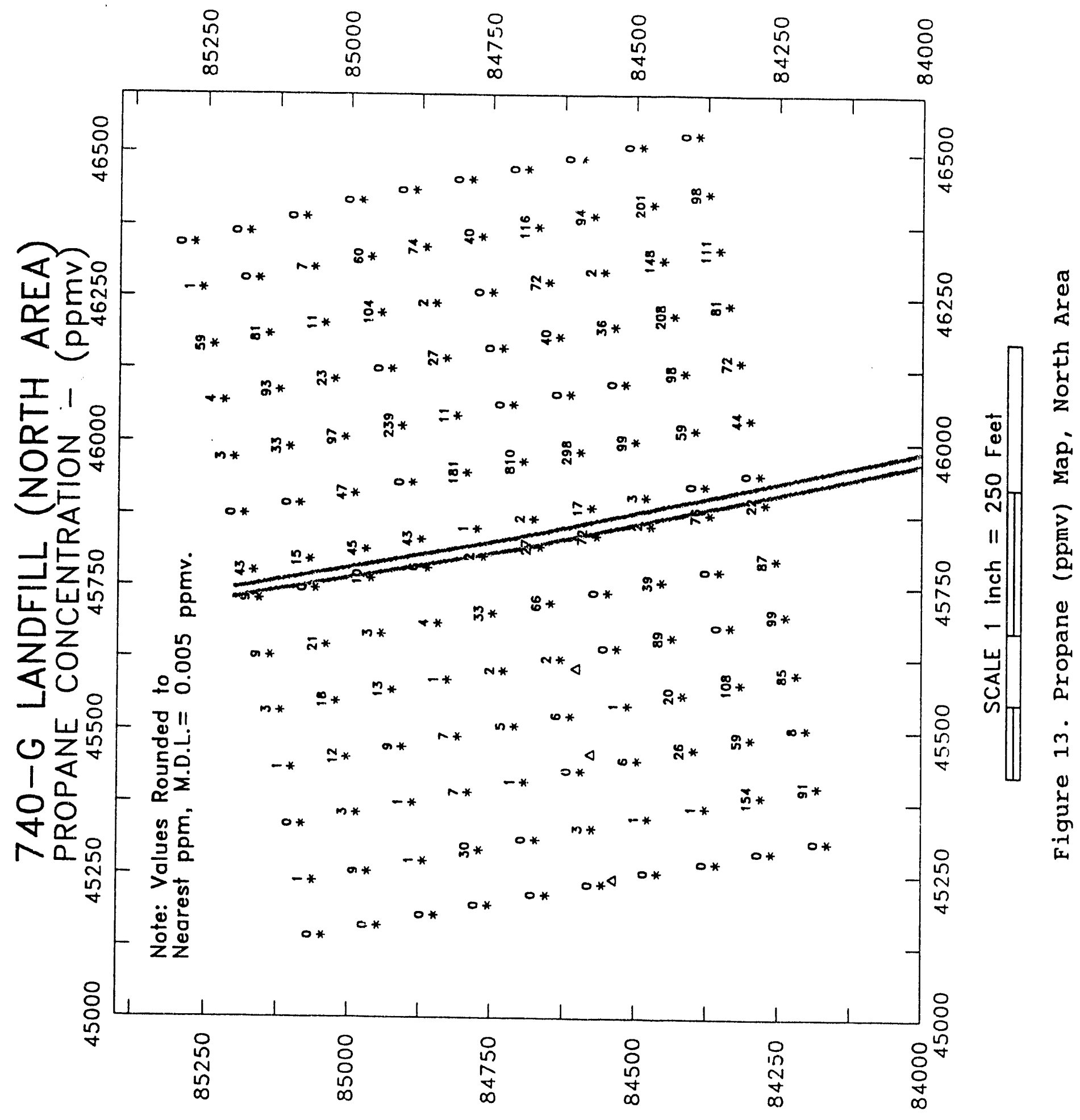




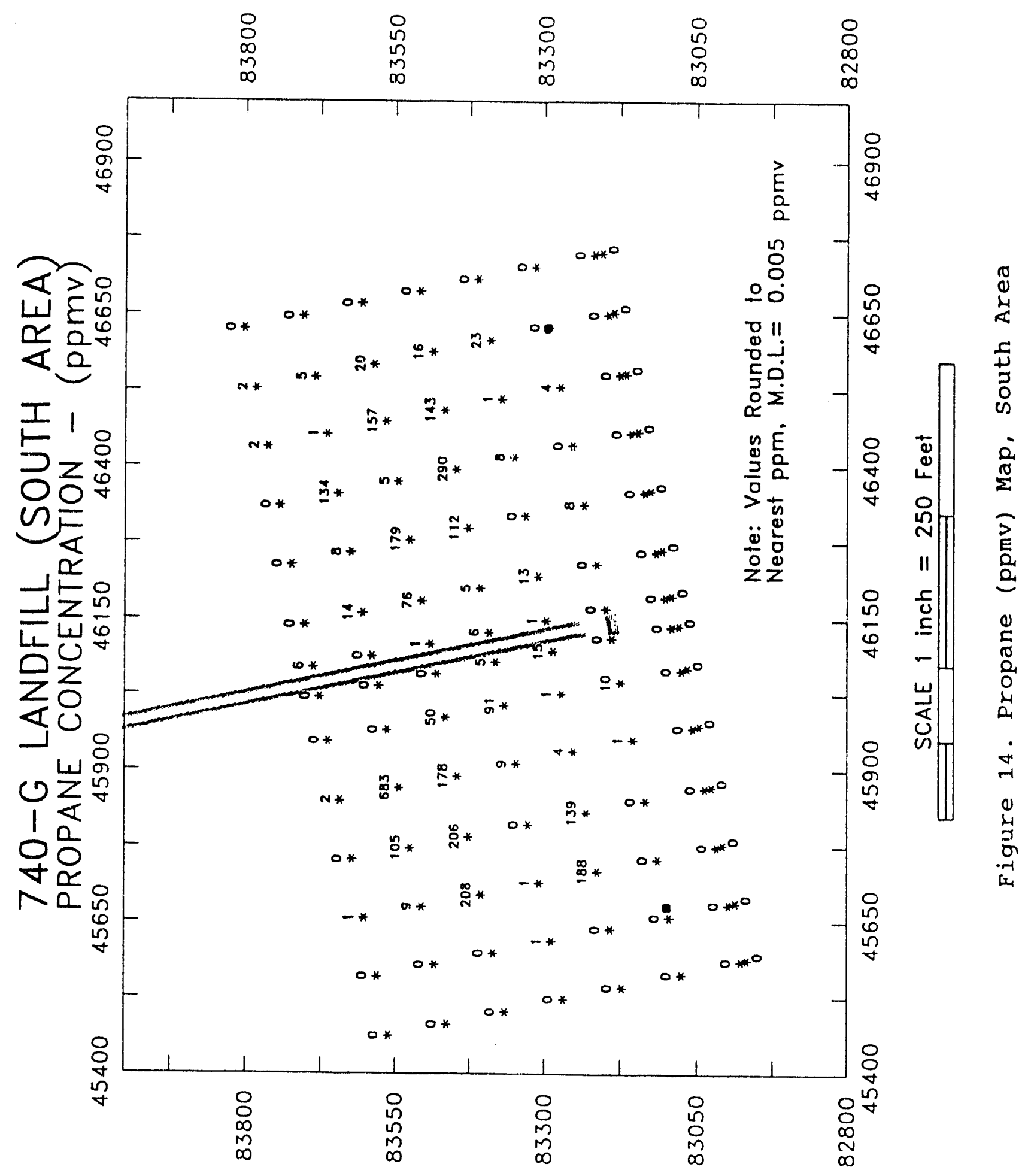




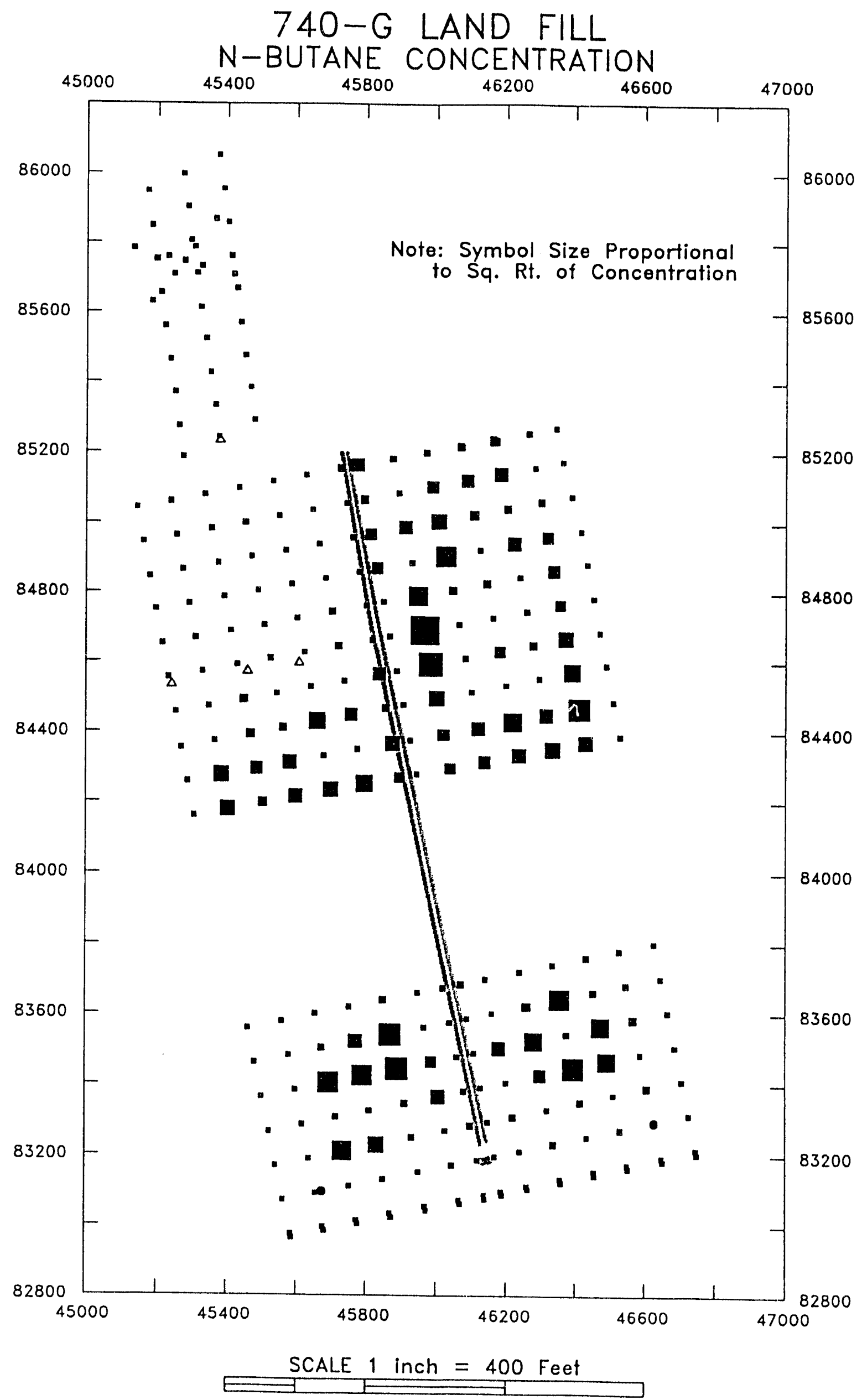

Figure 15. n-Butane symbol Map 


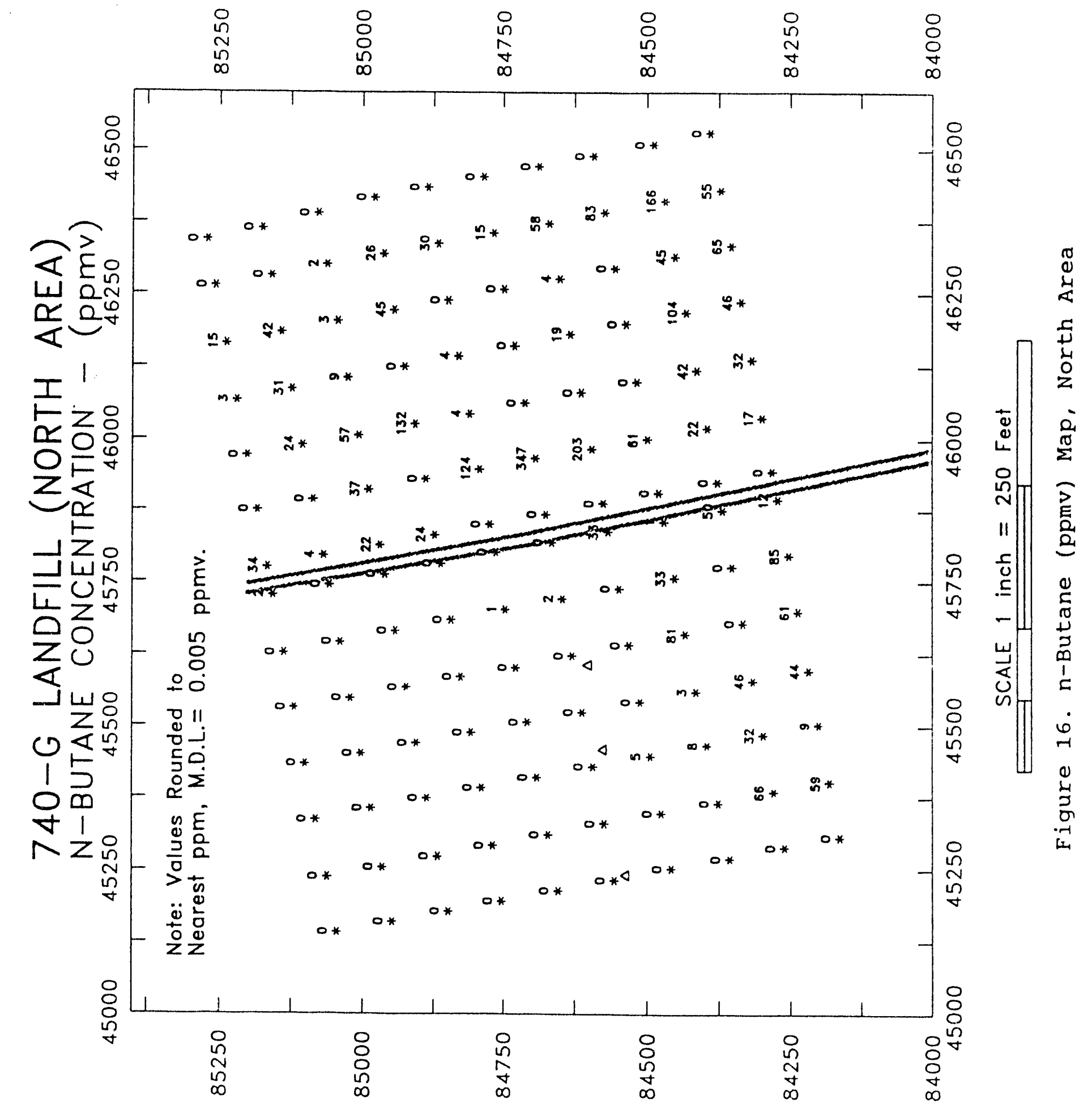




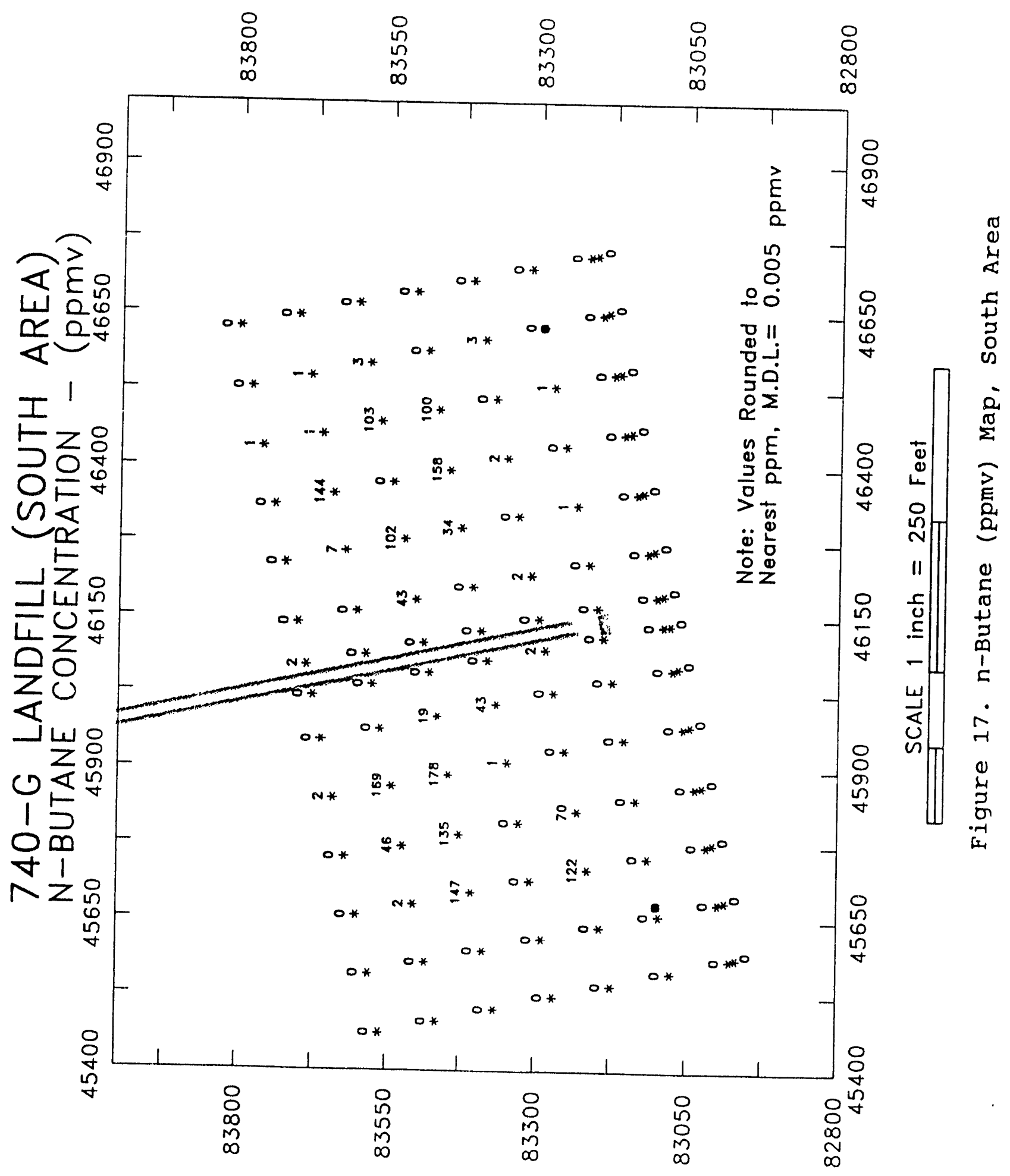




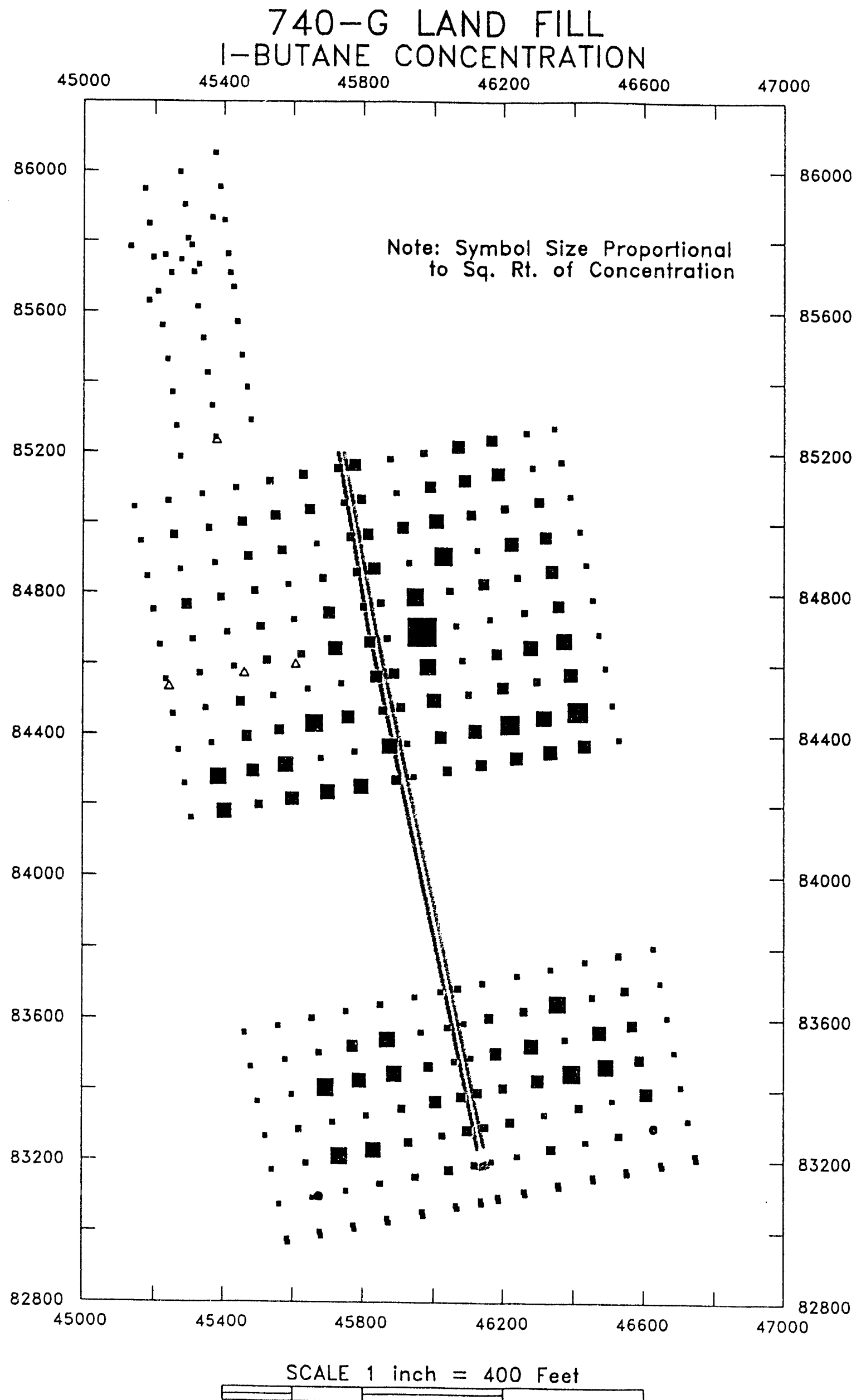

Figure 18. i-Butane symbol Map 


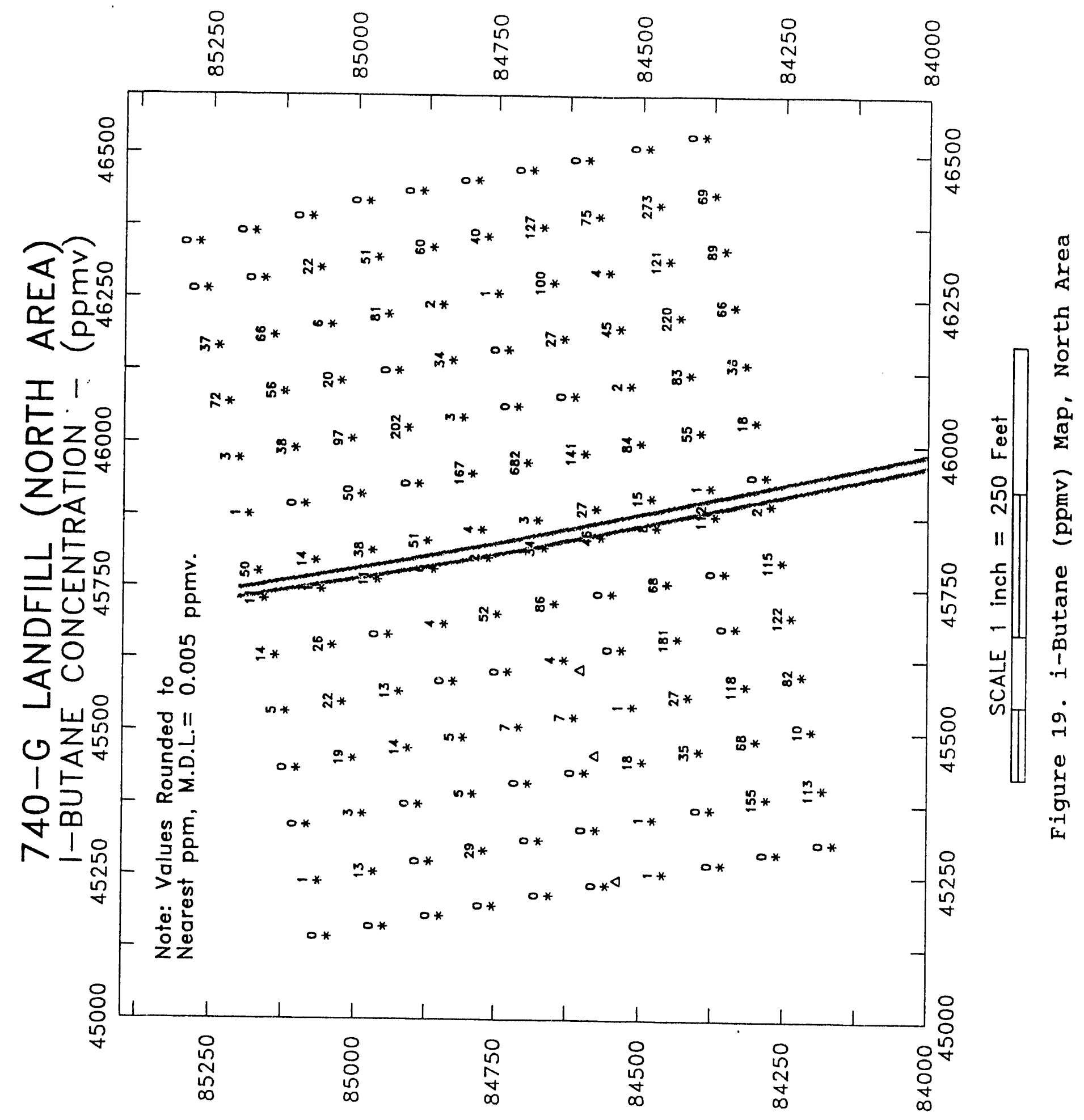




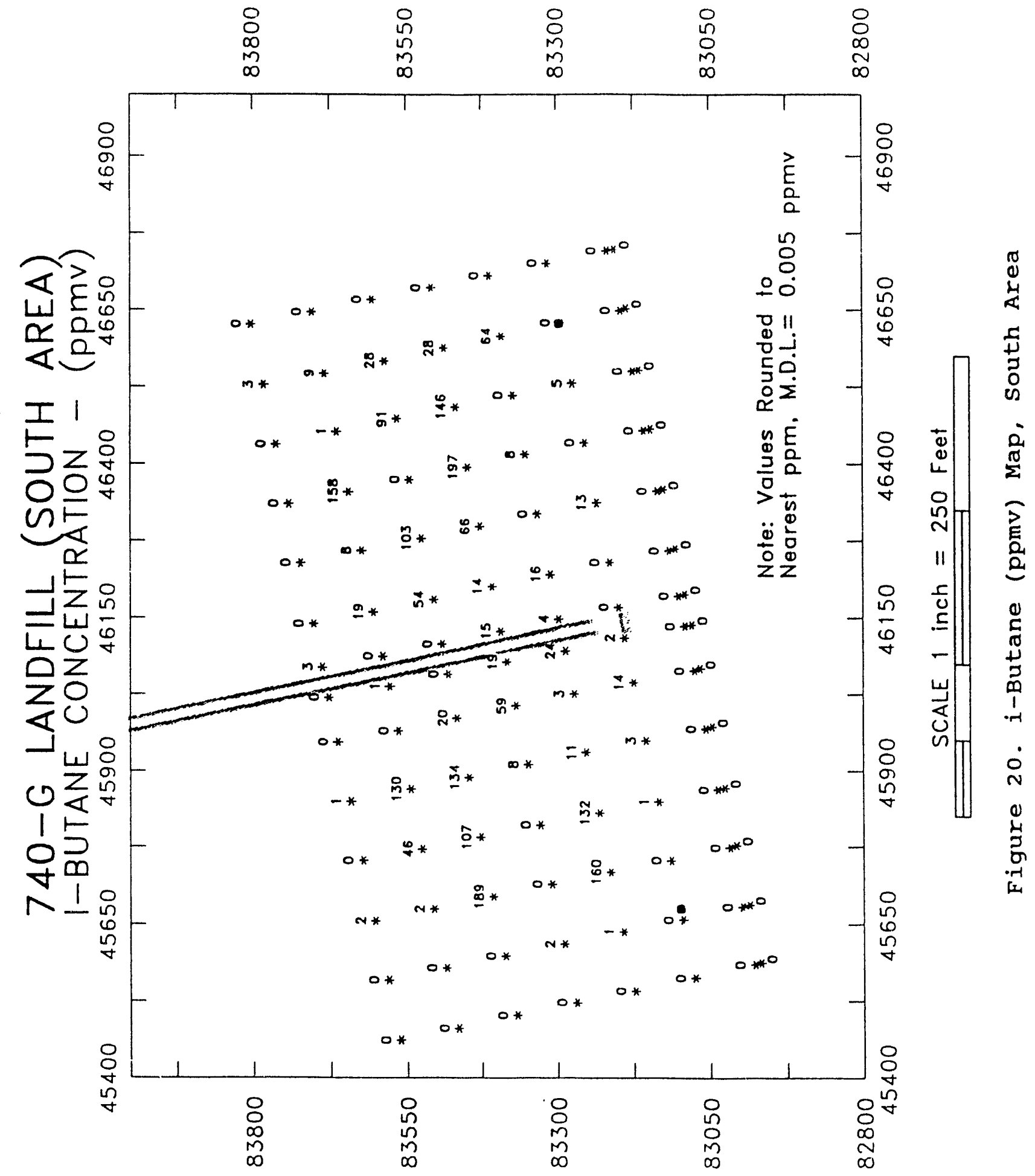




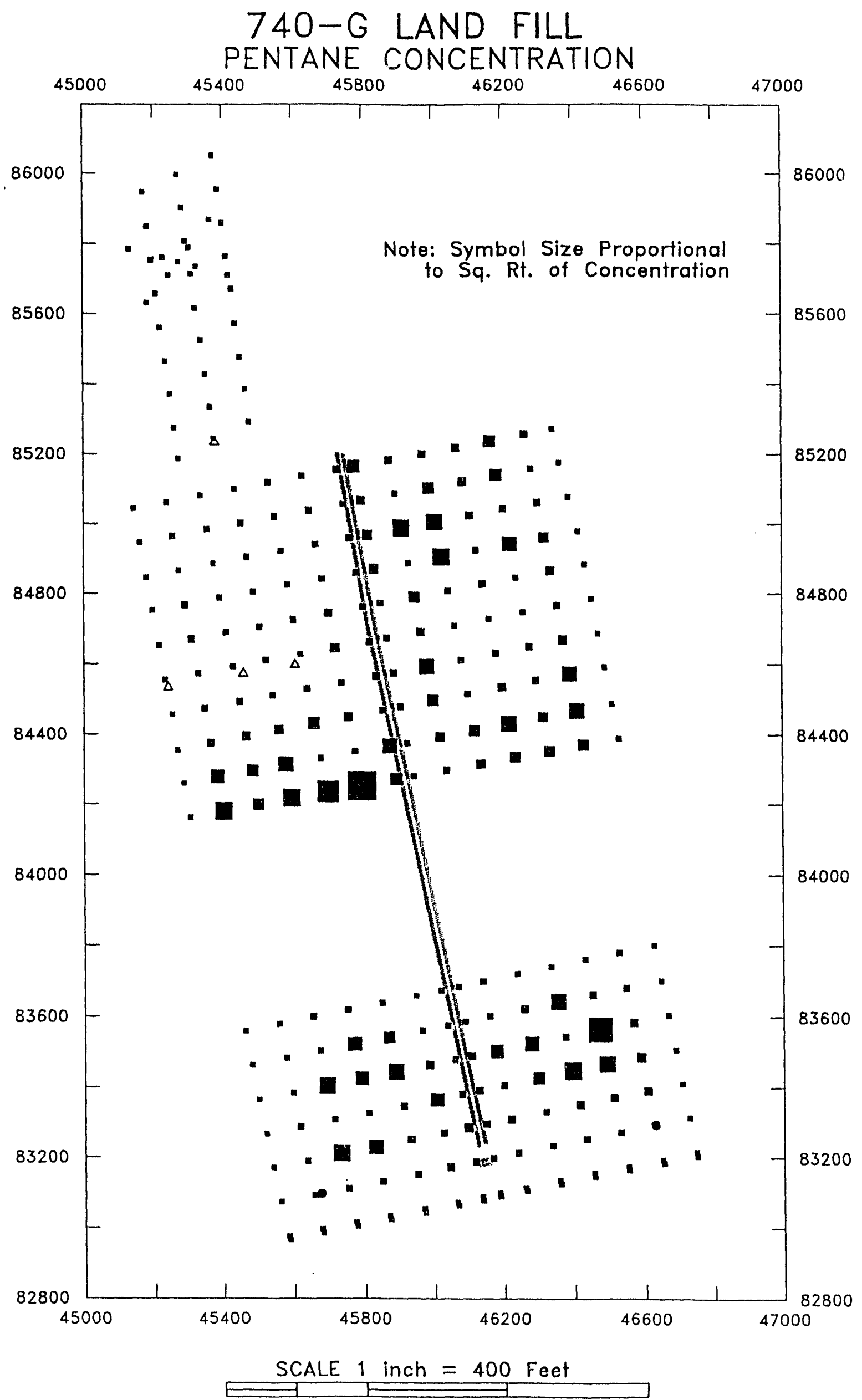

Figure 21. Pentane Symbol Map 


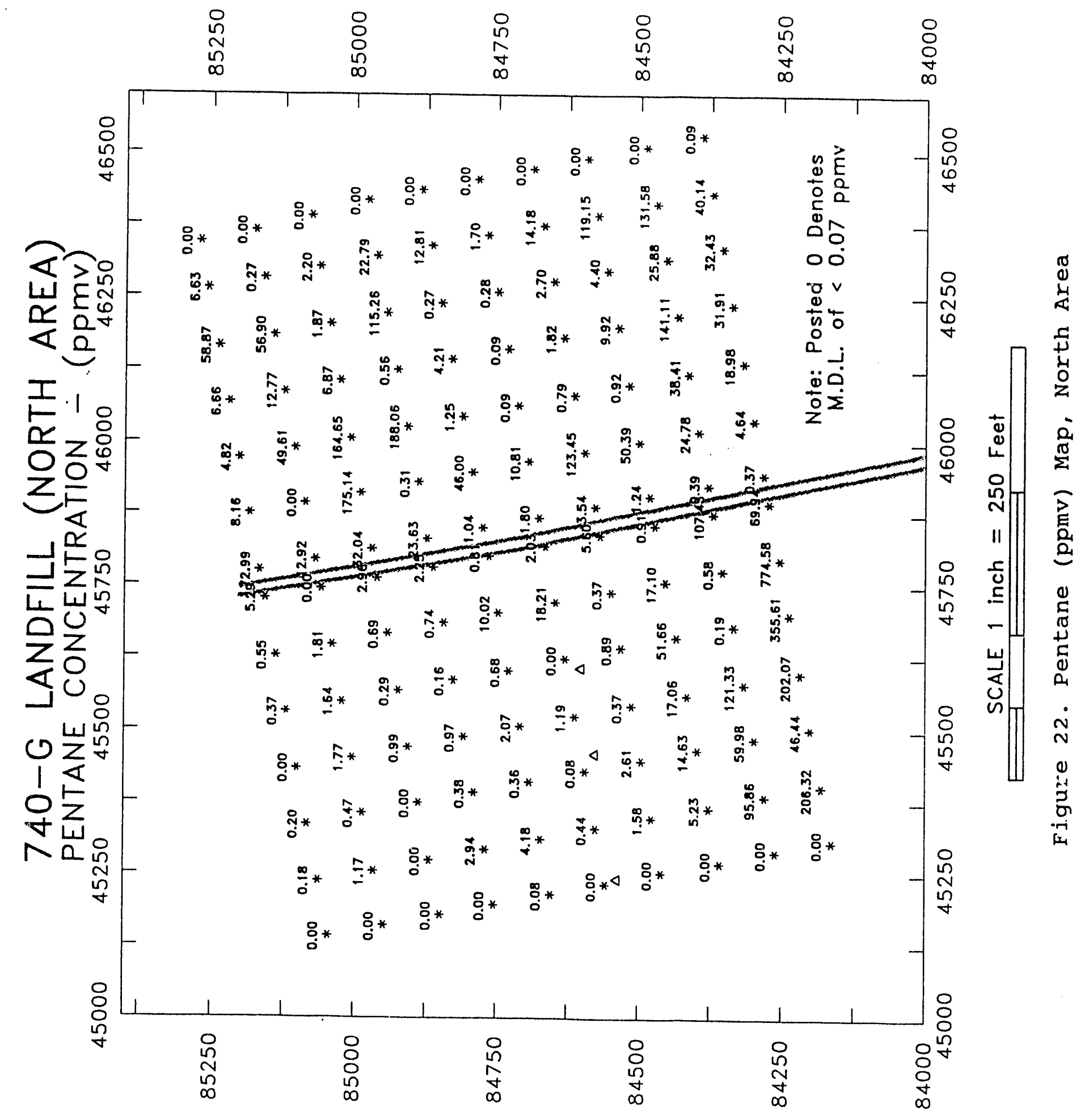




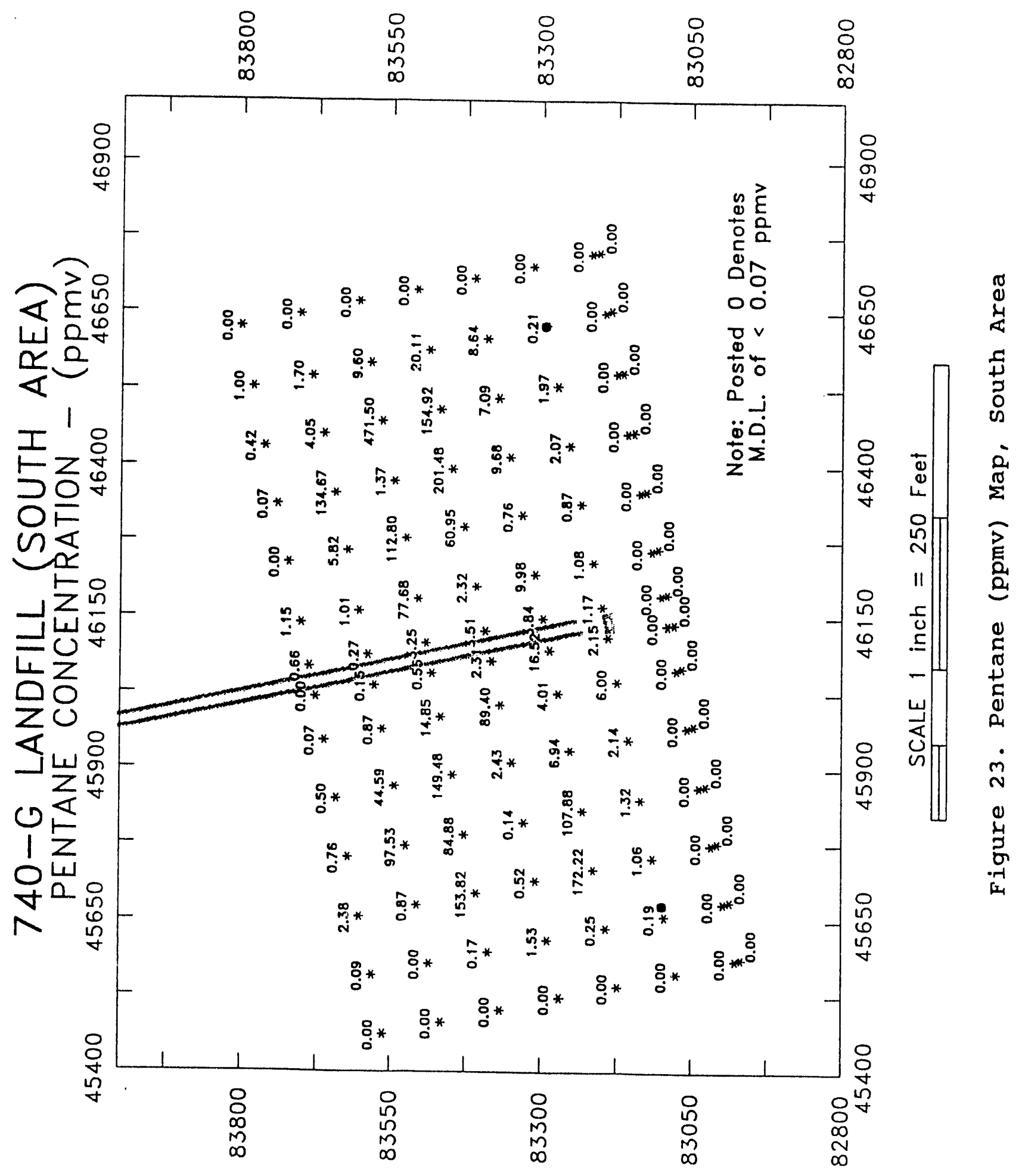




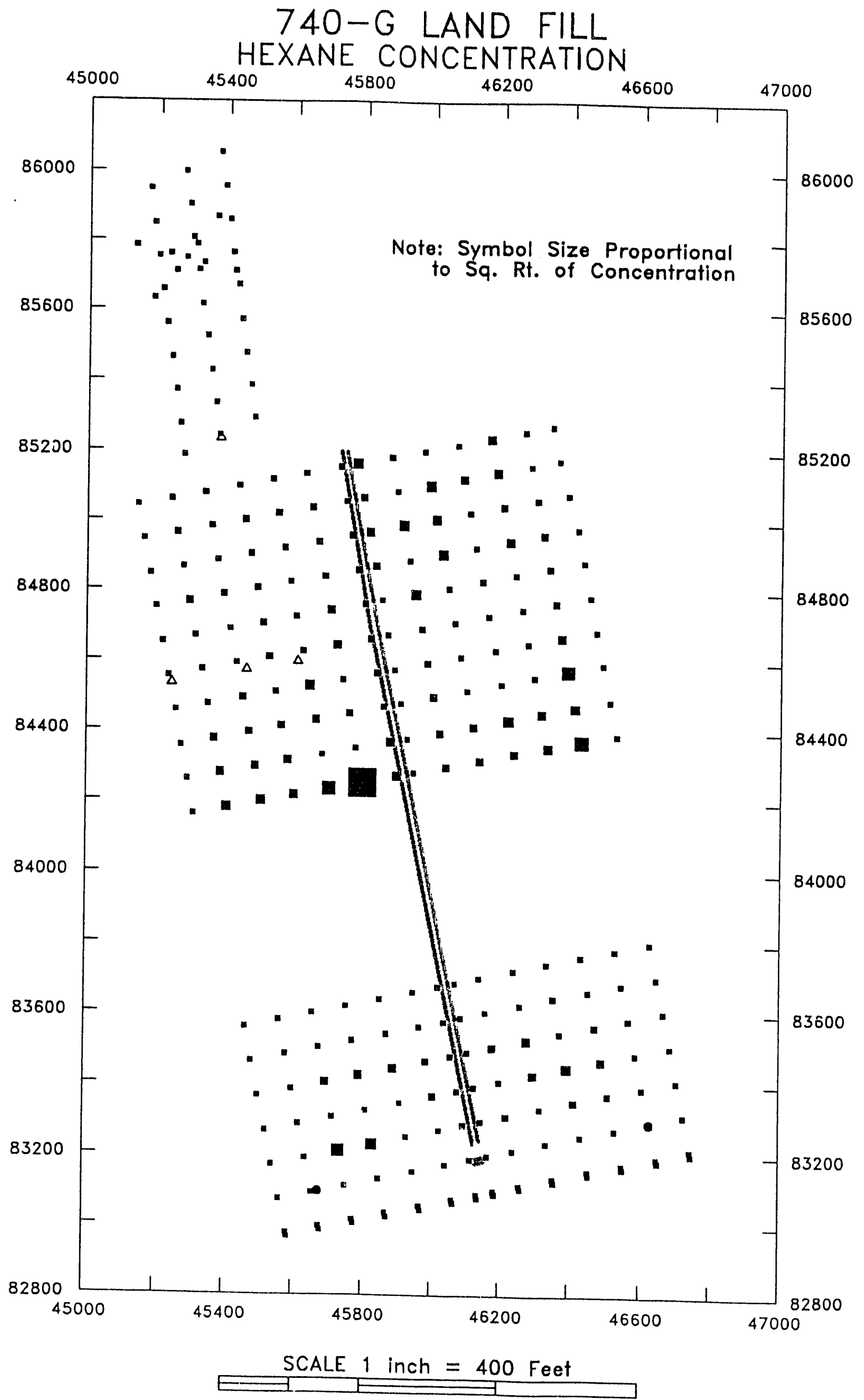

Figure 24. Hexane Symbol Map 


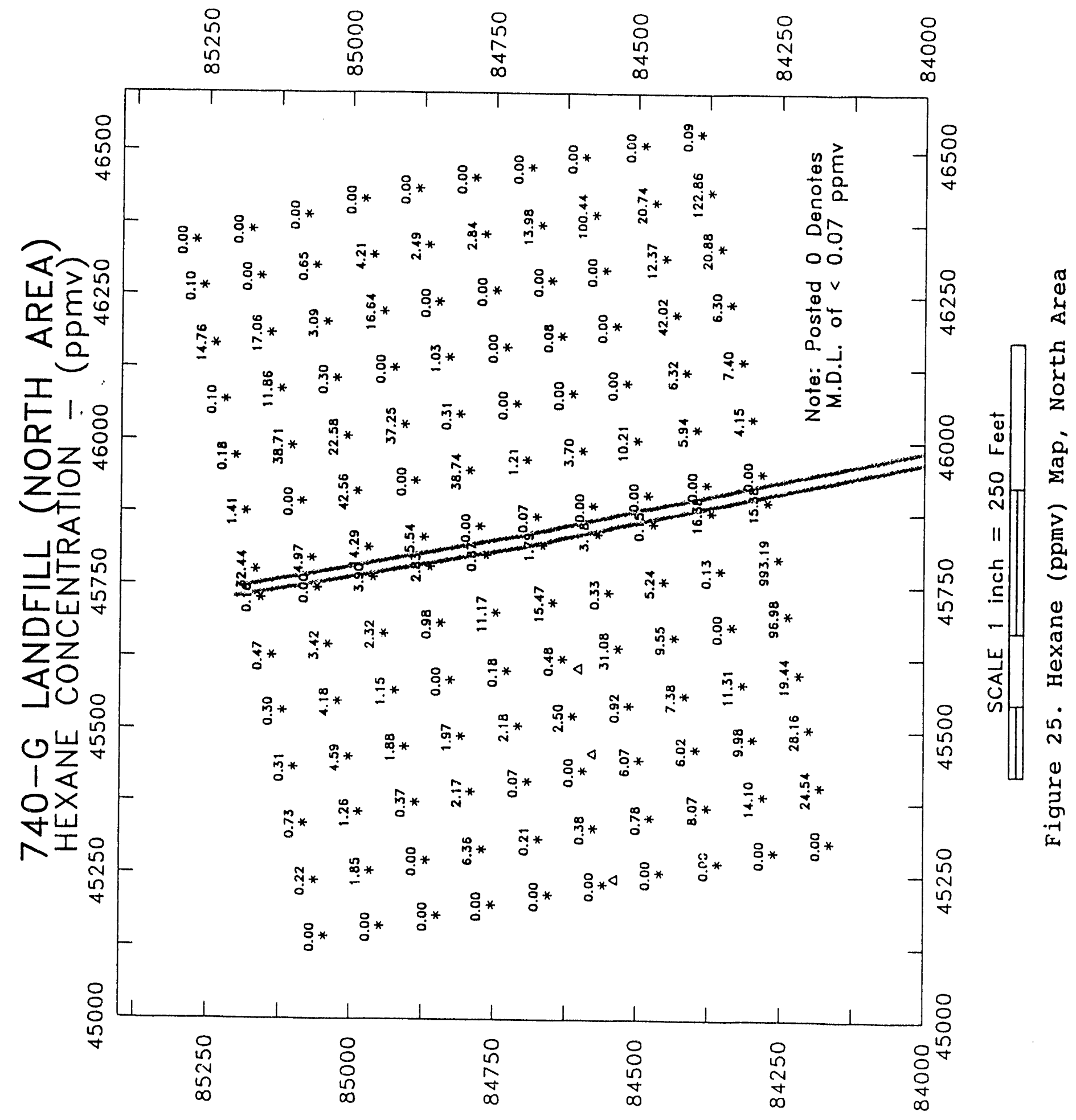




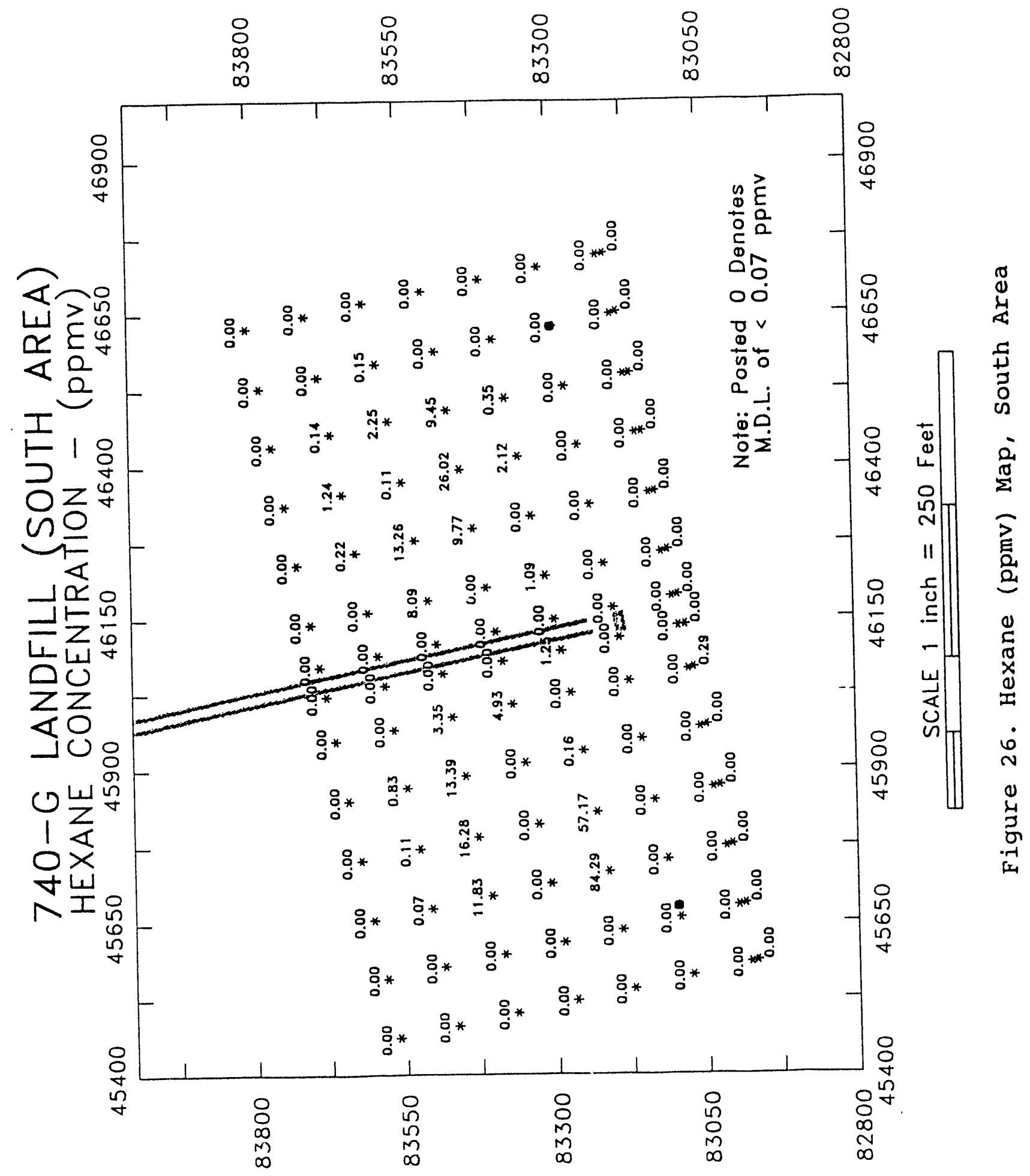




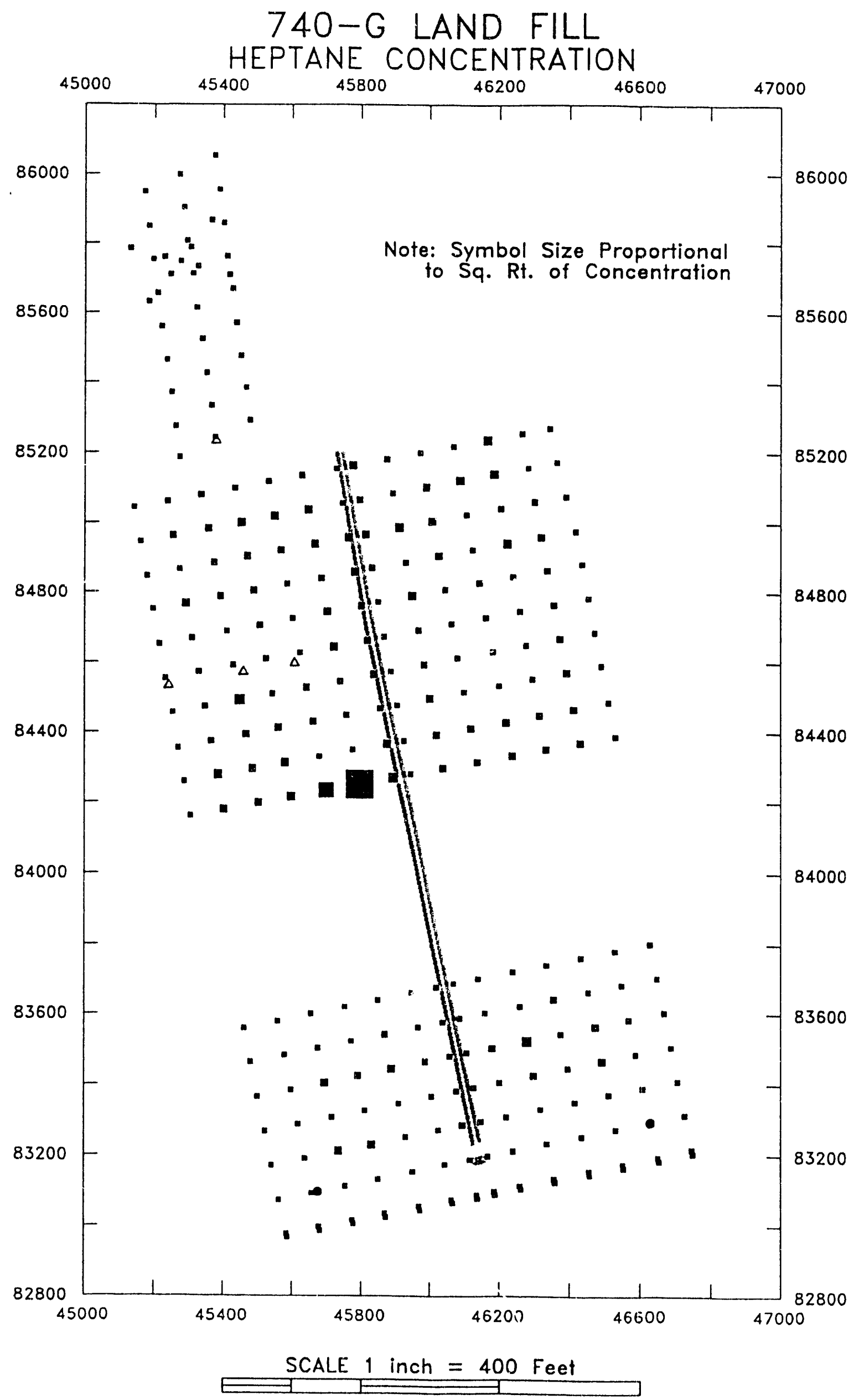

Figure 27. Heptane Symbol Map 


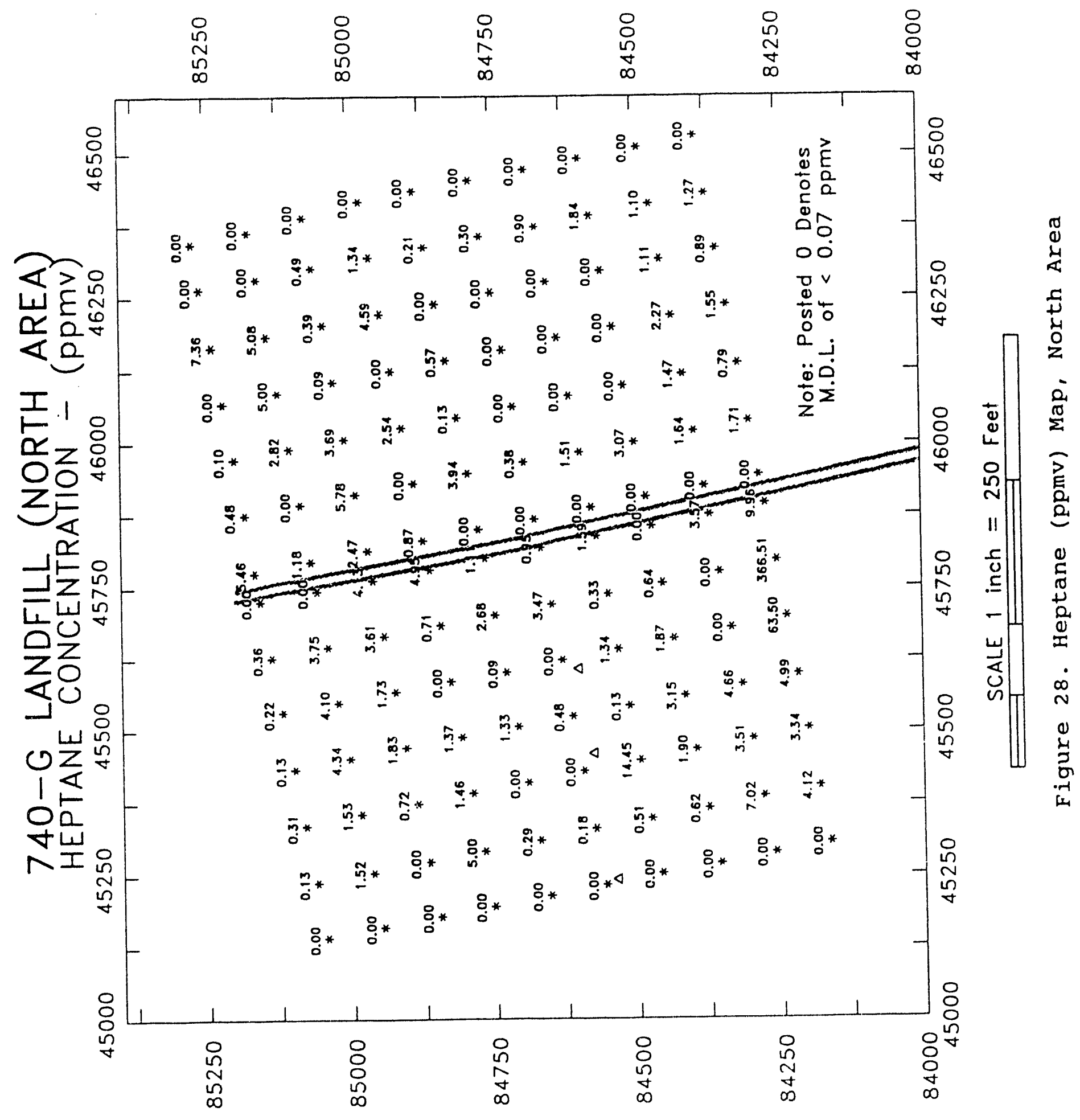




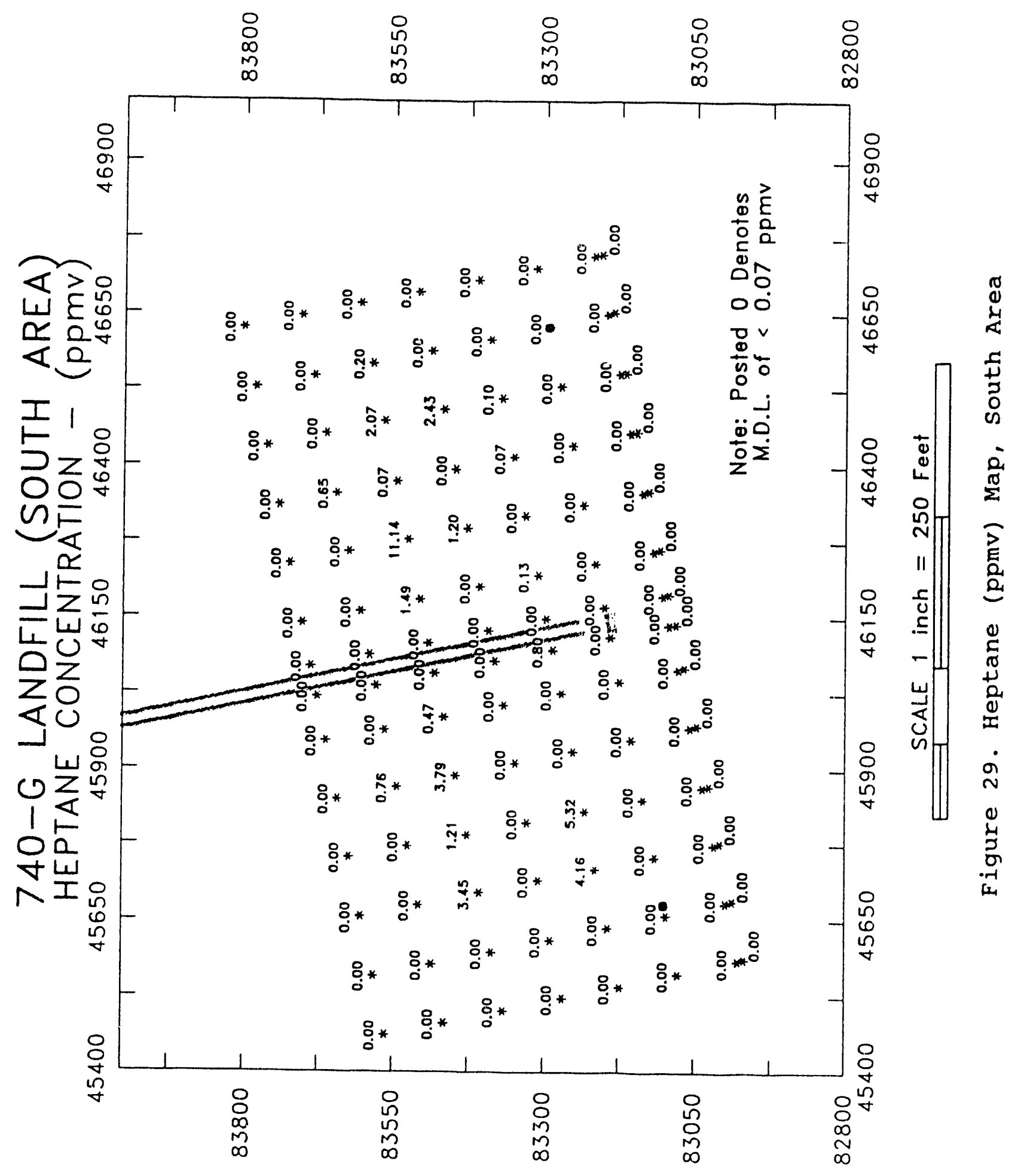




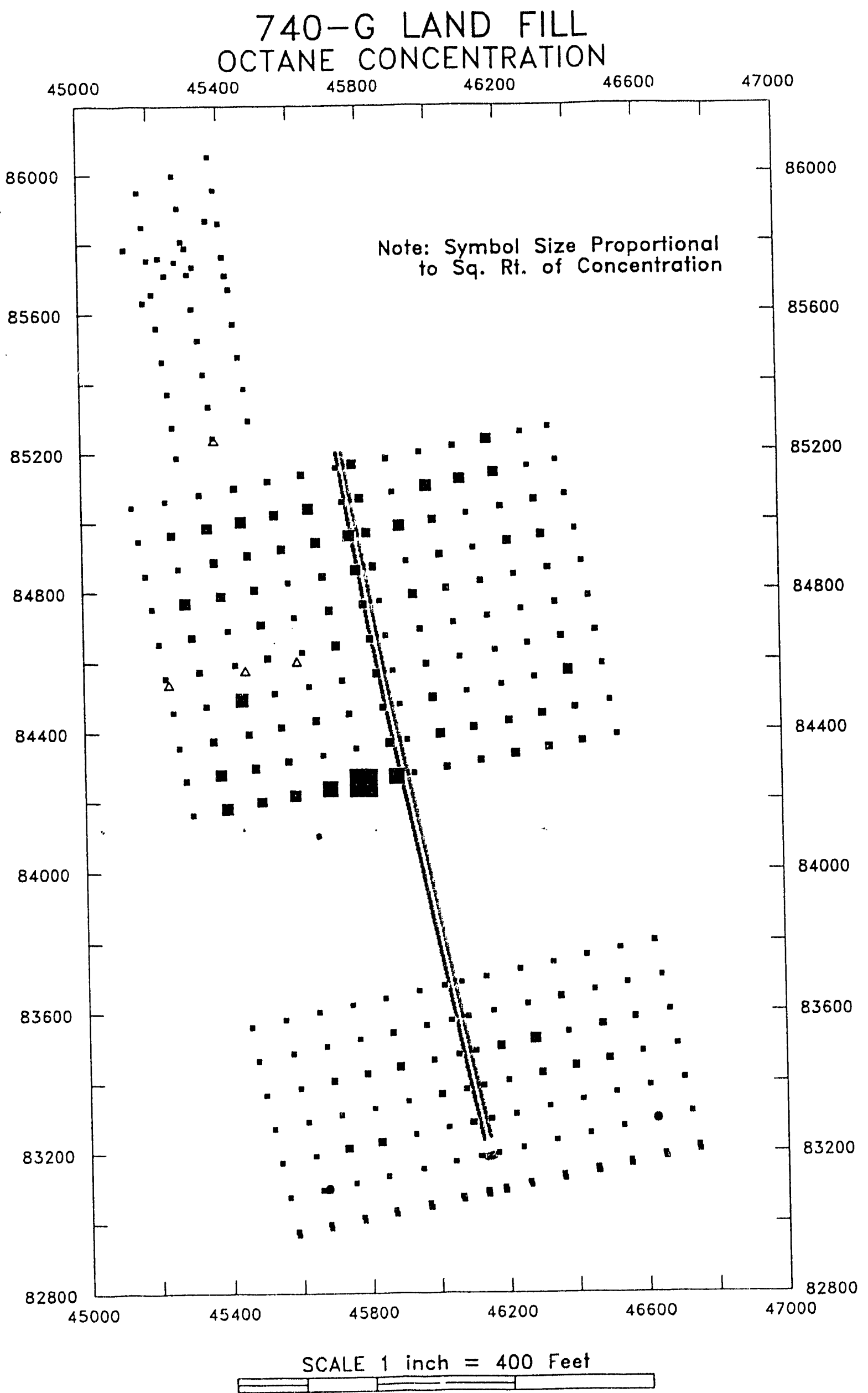

Figure 30. Octane Symbol Map 


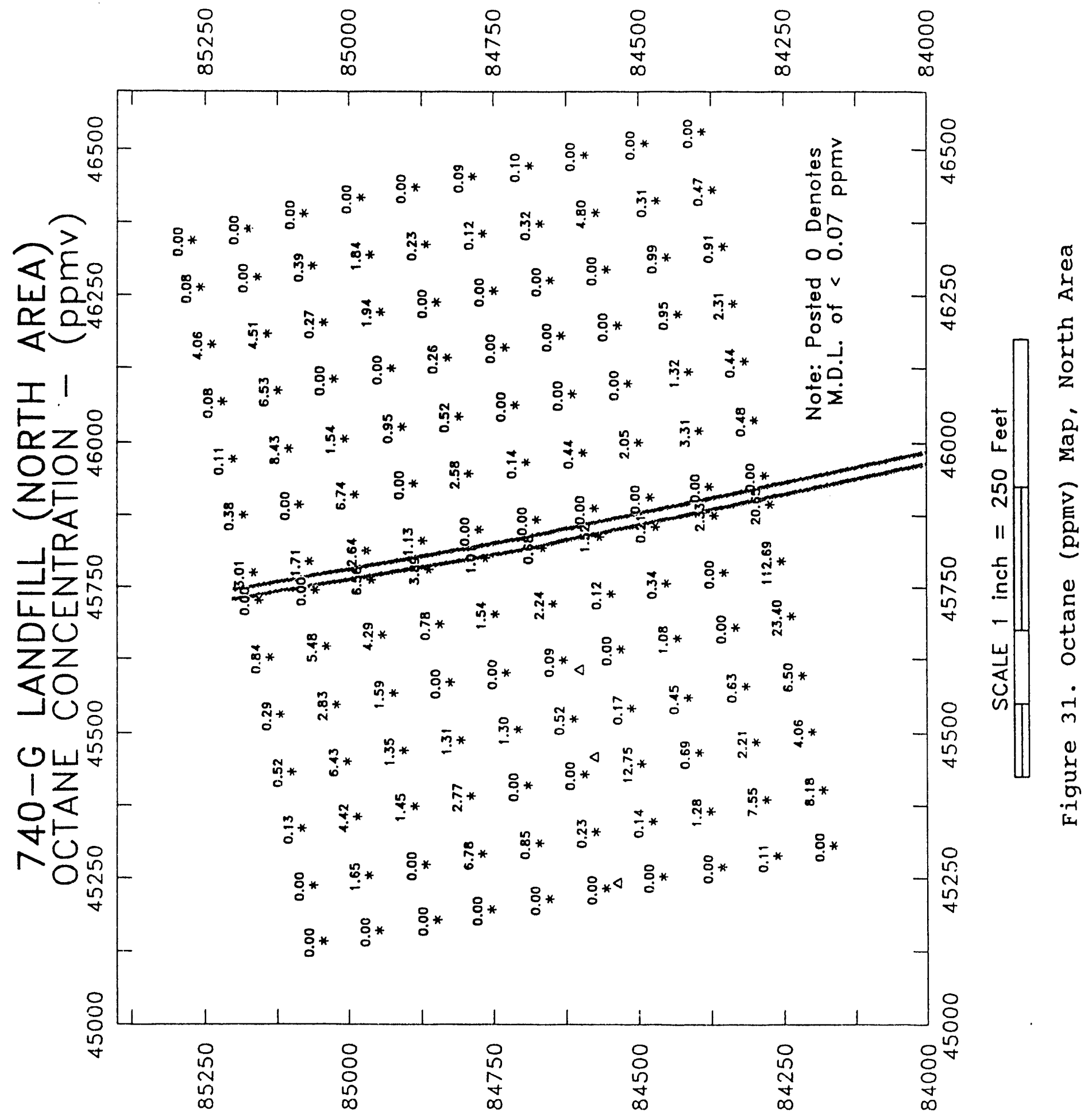




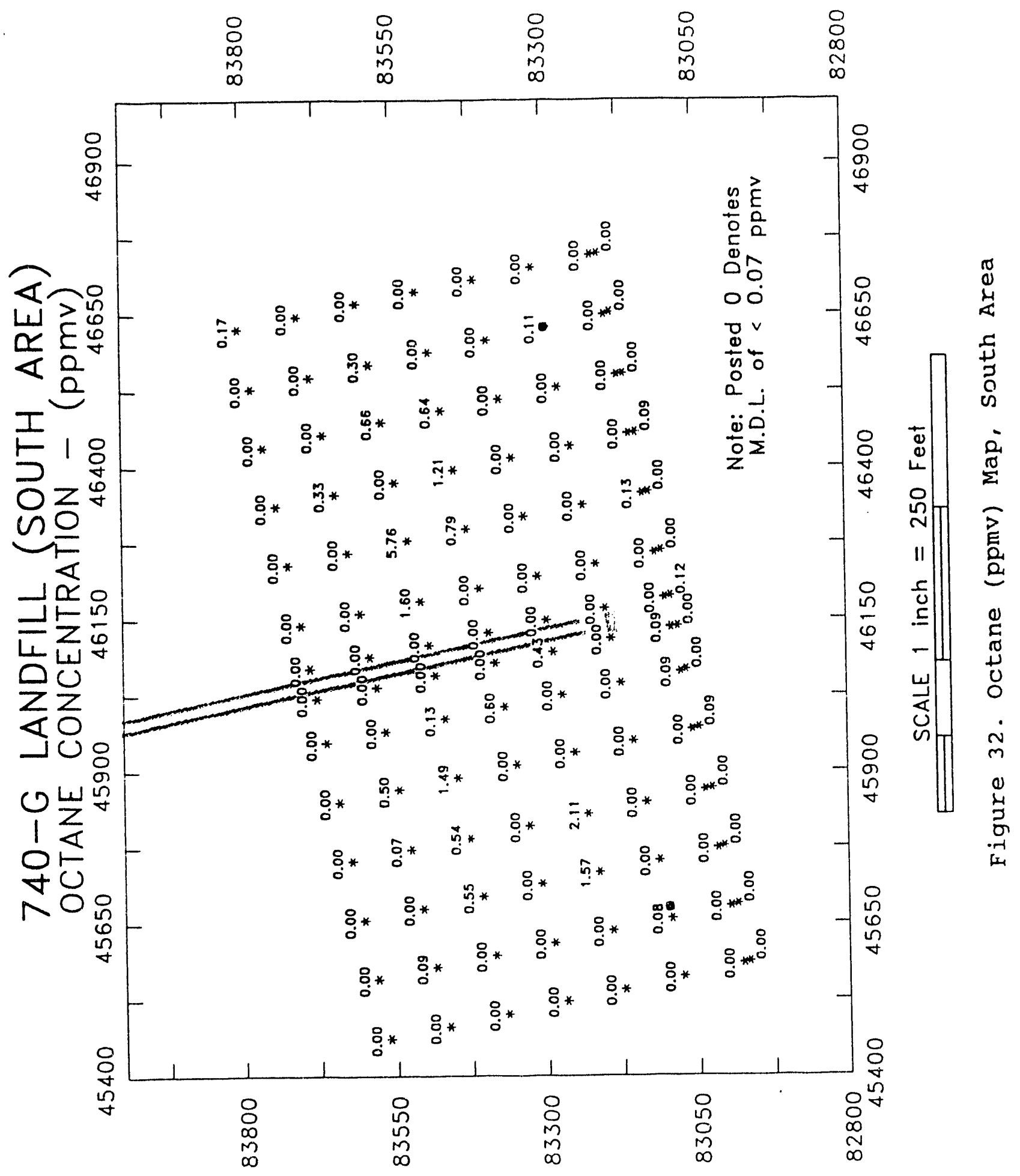




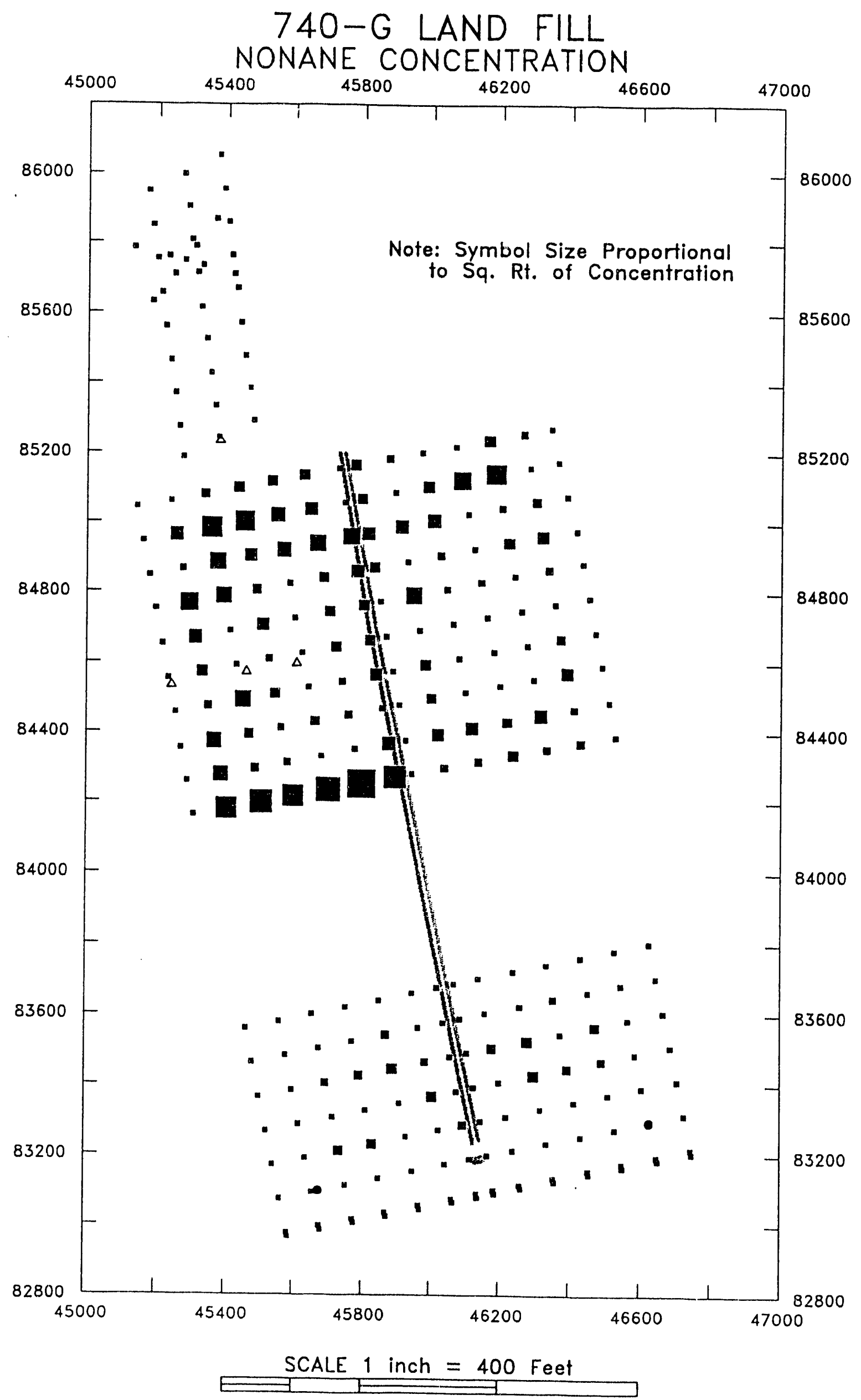

Figure 33. Nonane Symbol Map 


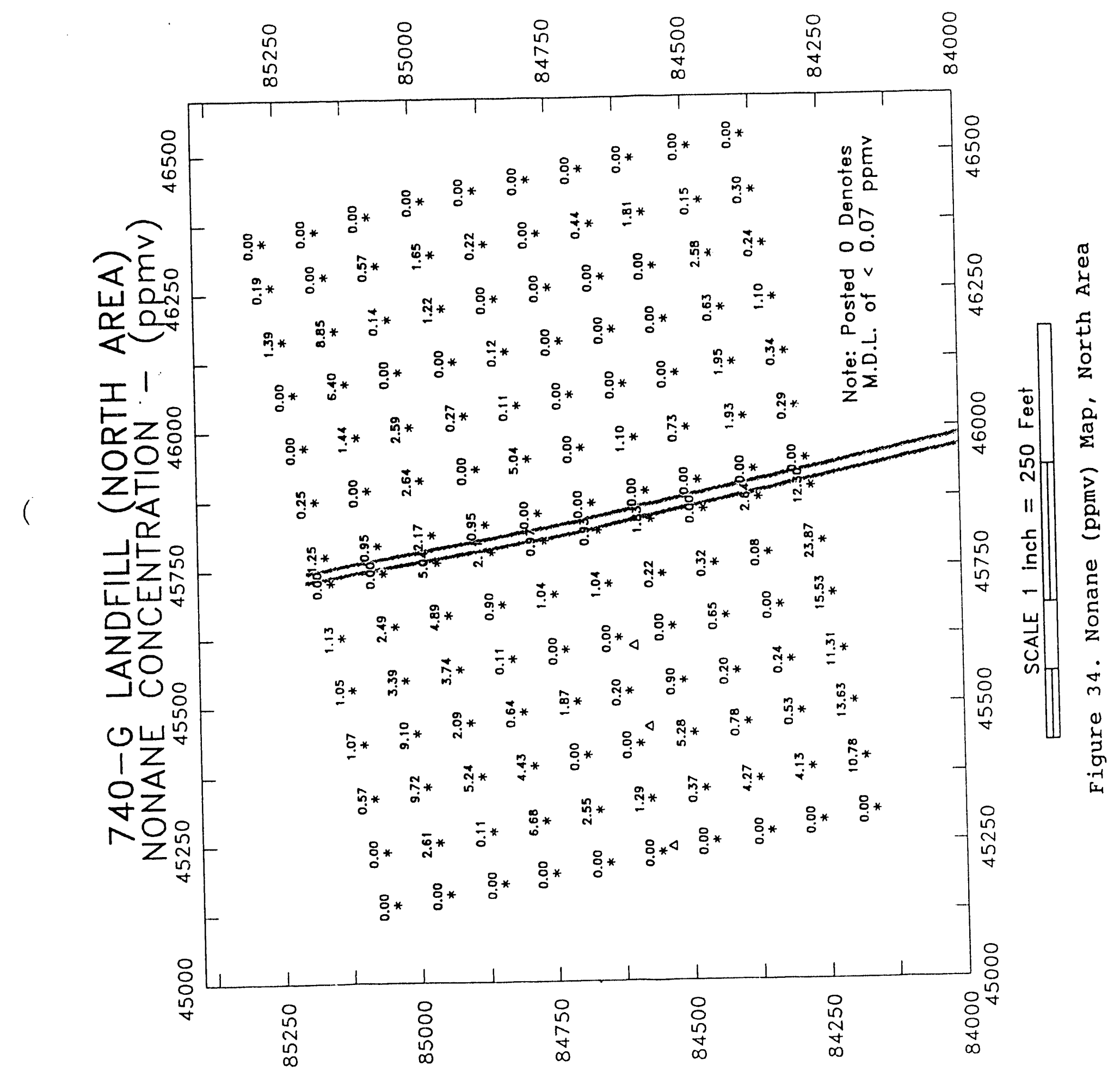




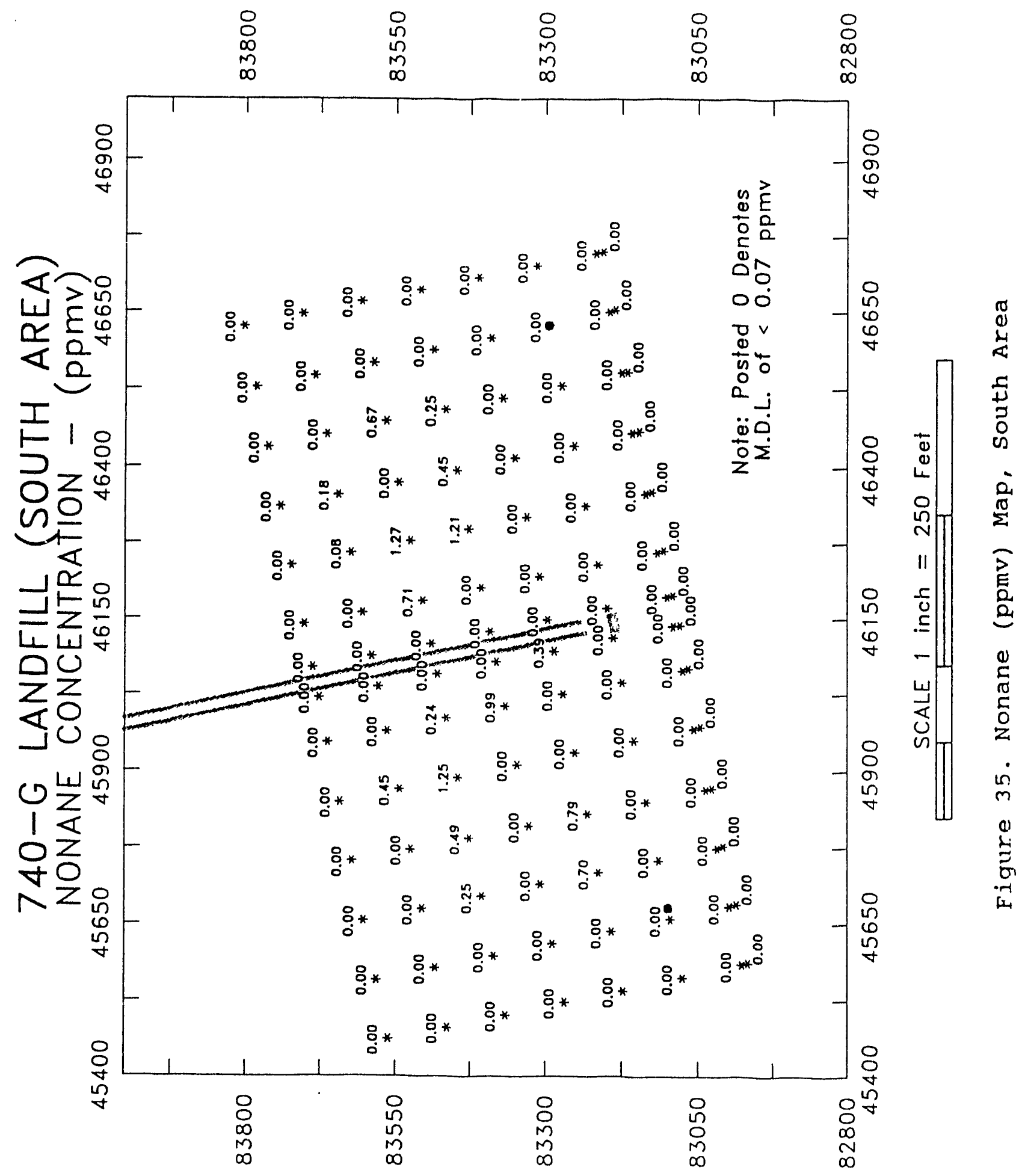




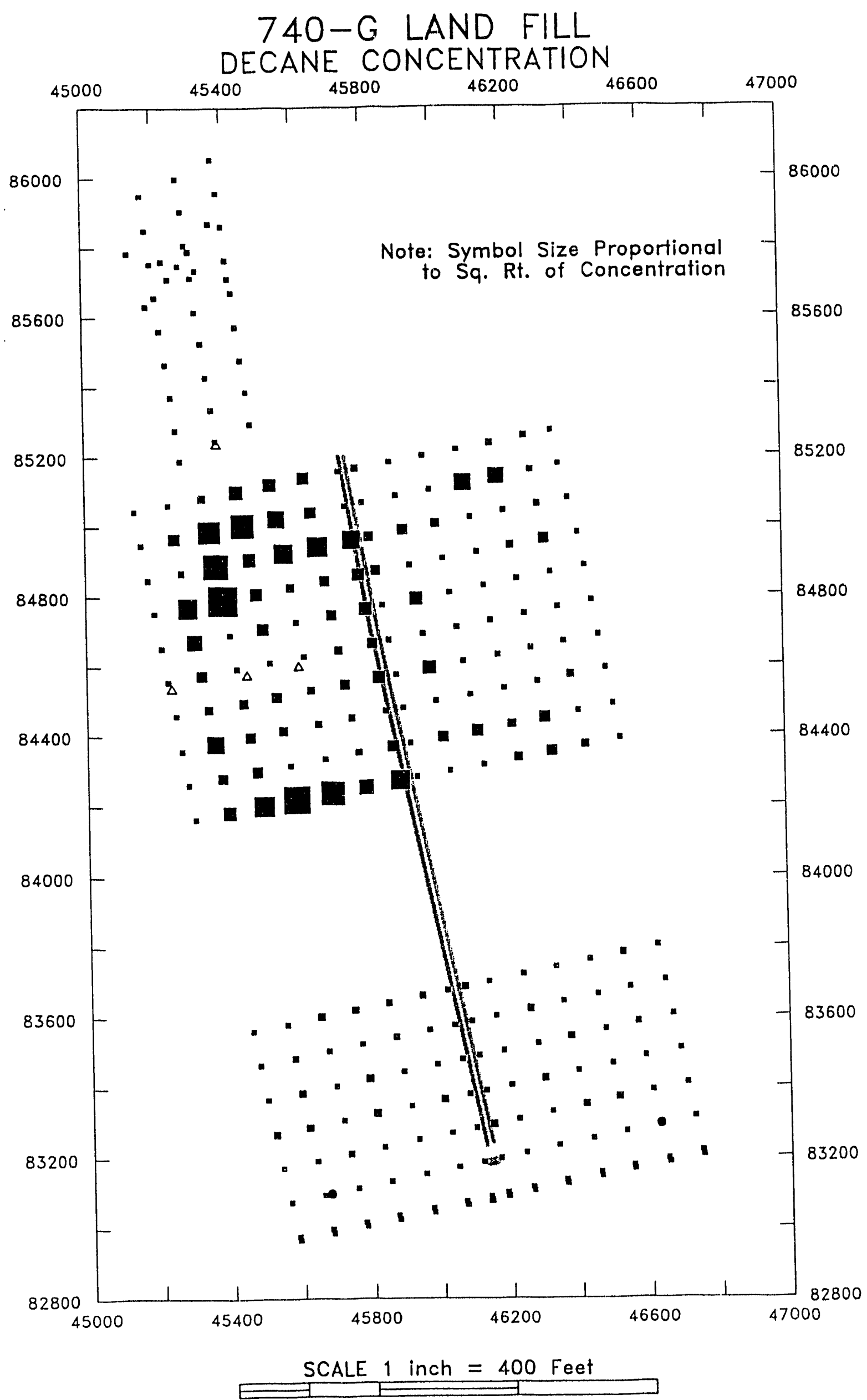

Figure 36. Decane Symbol Map 


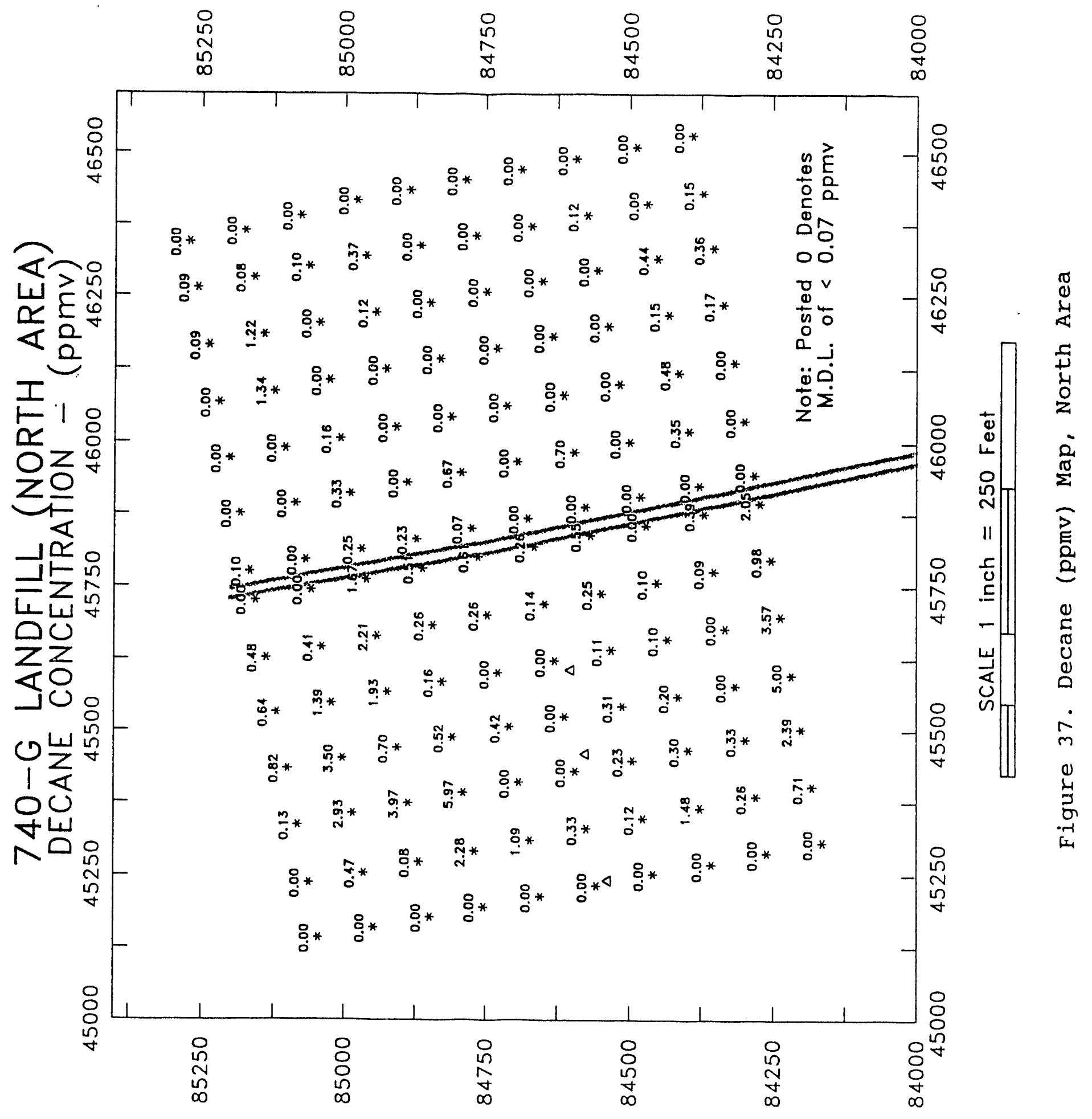




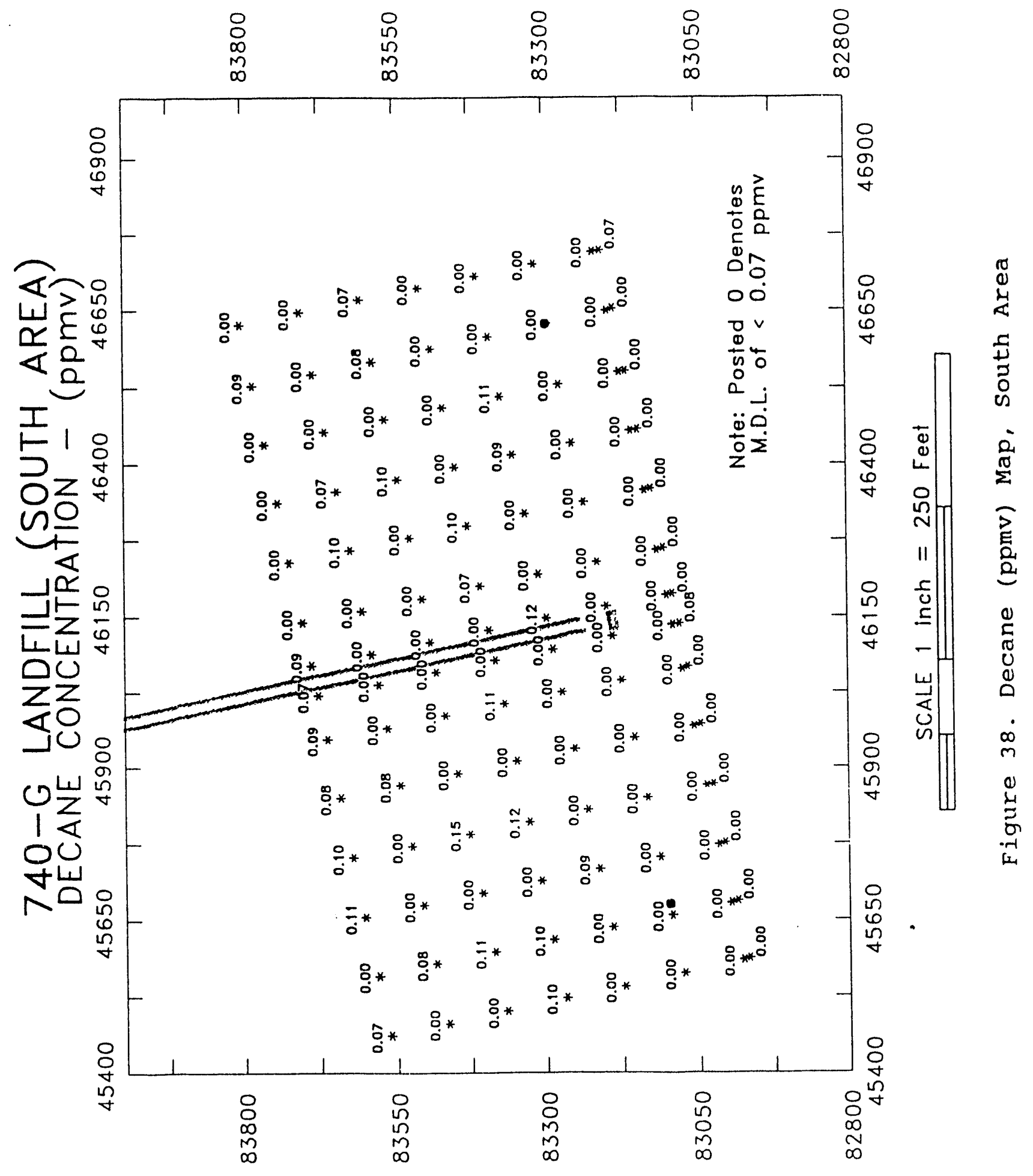




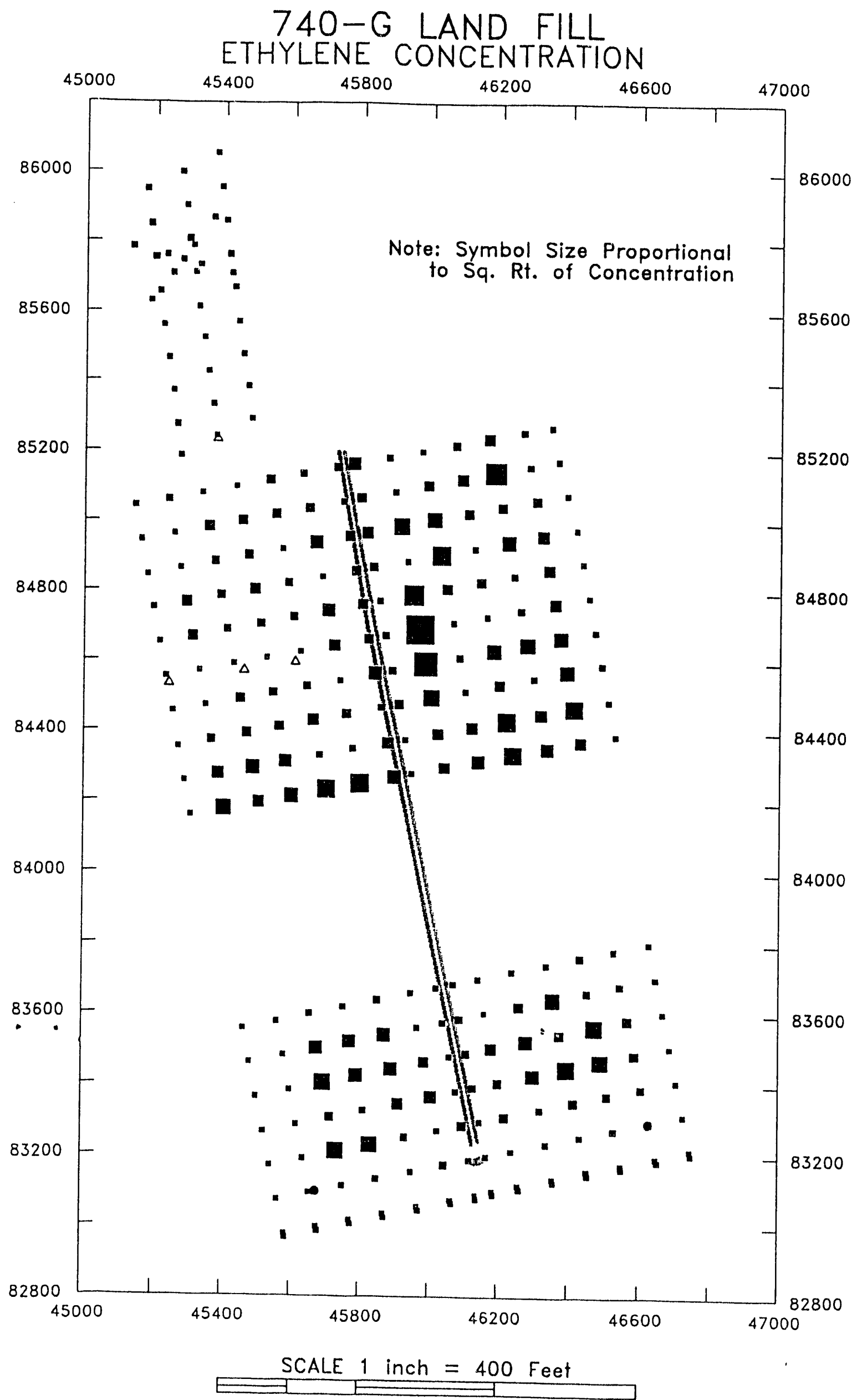

Figure 39. Ethylene symbol Map 


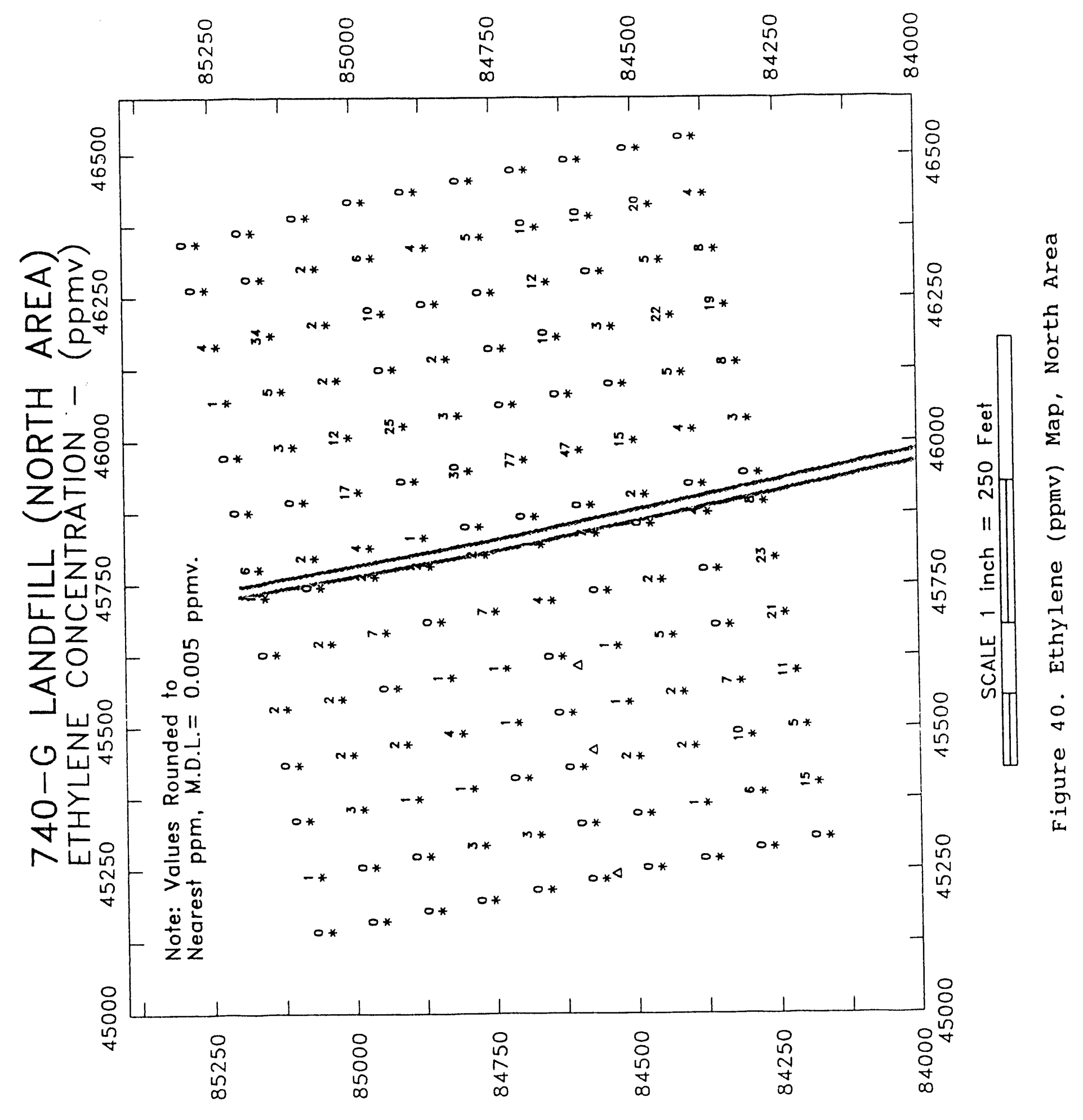




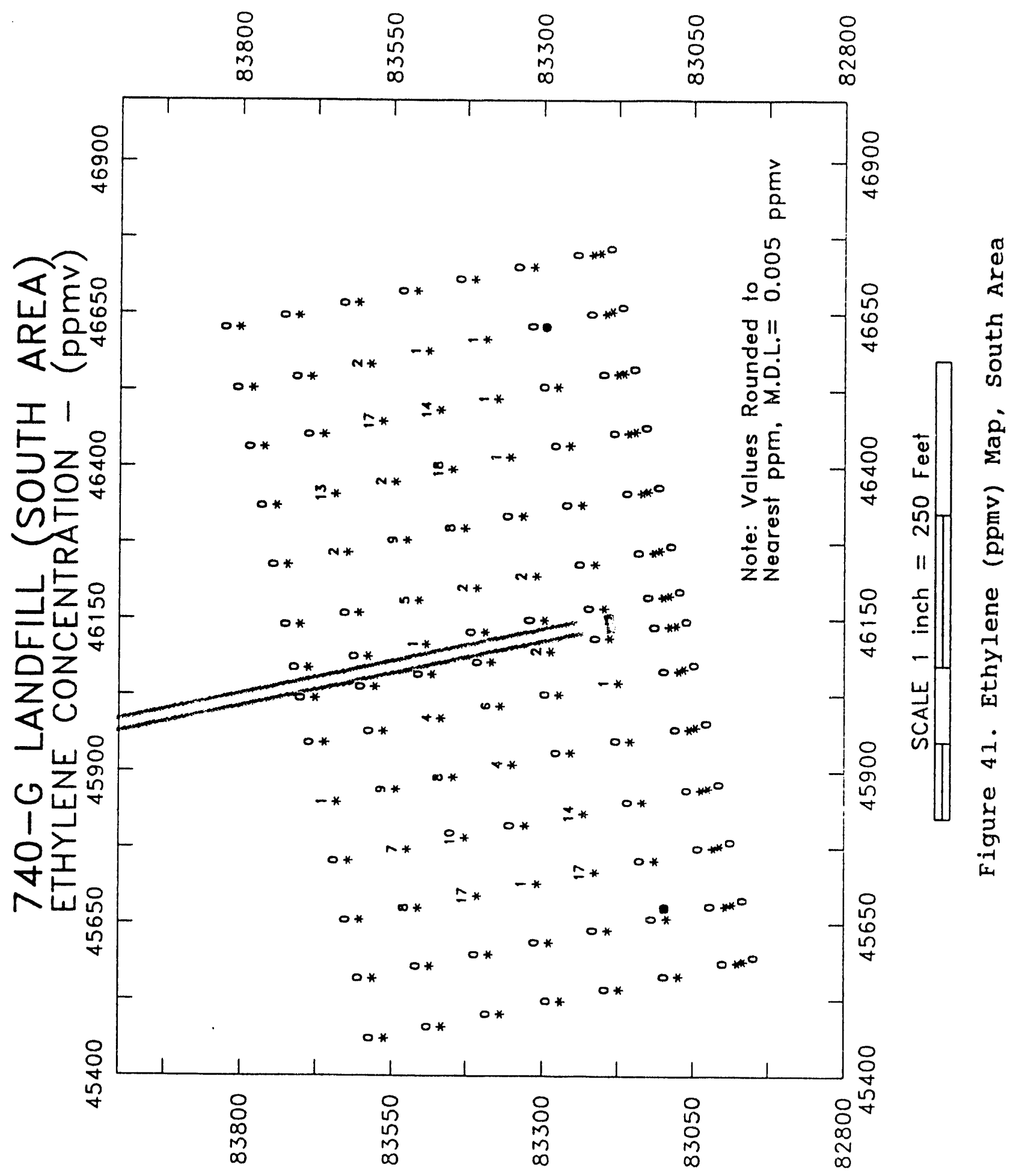




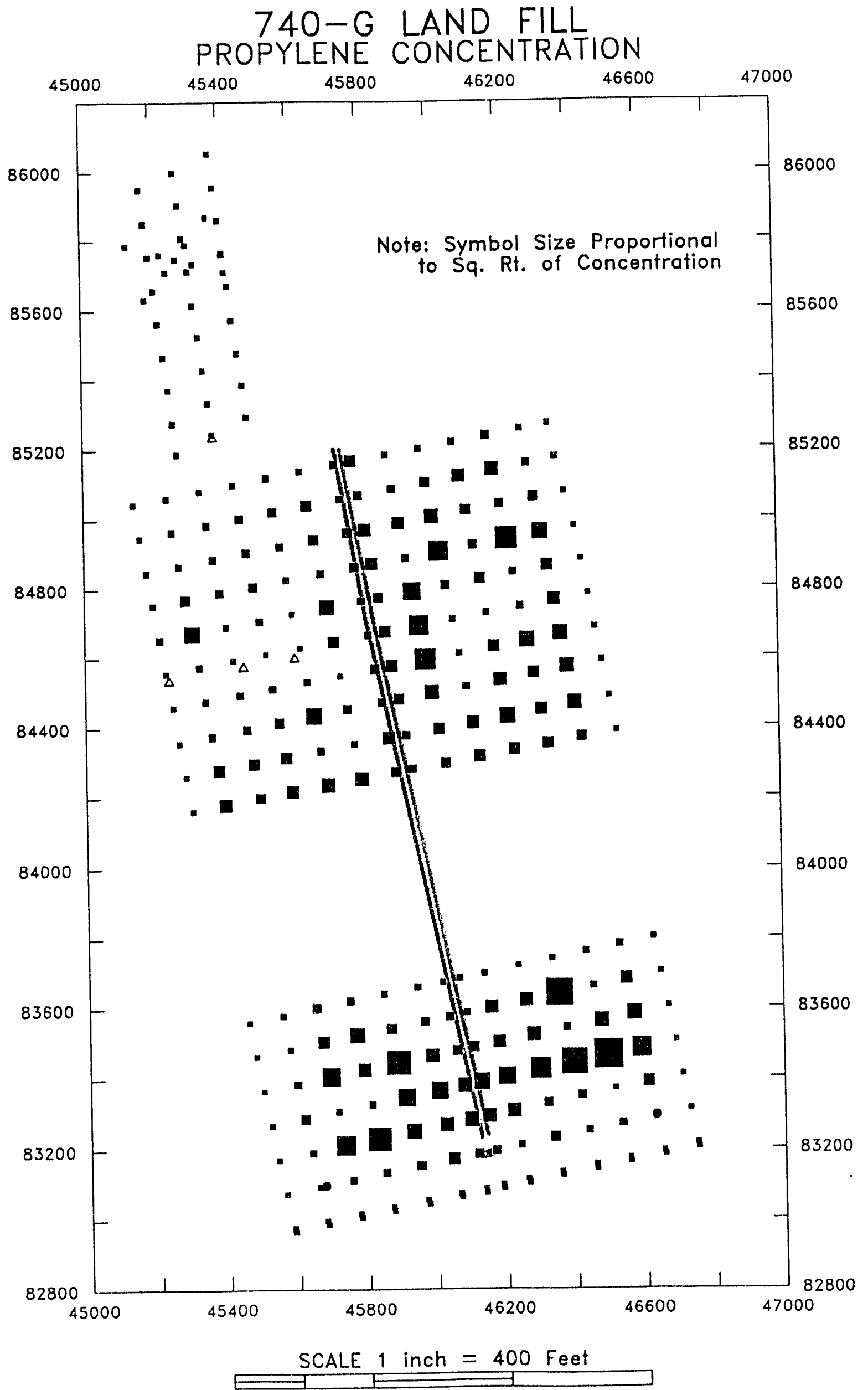

Figure 42. Propylene Symbol Map 


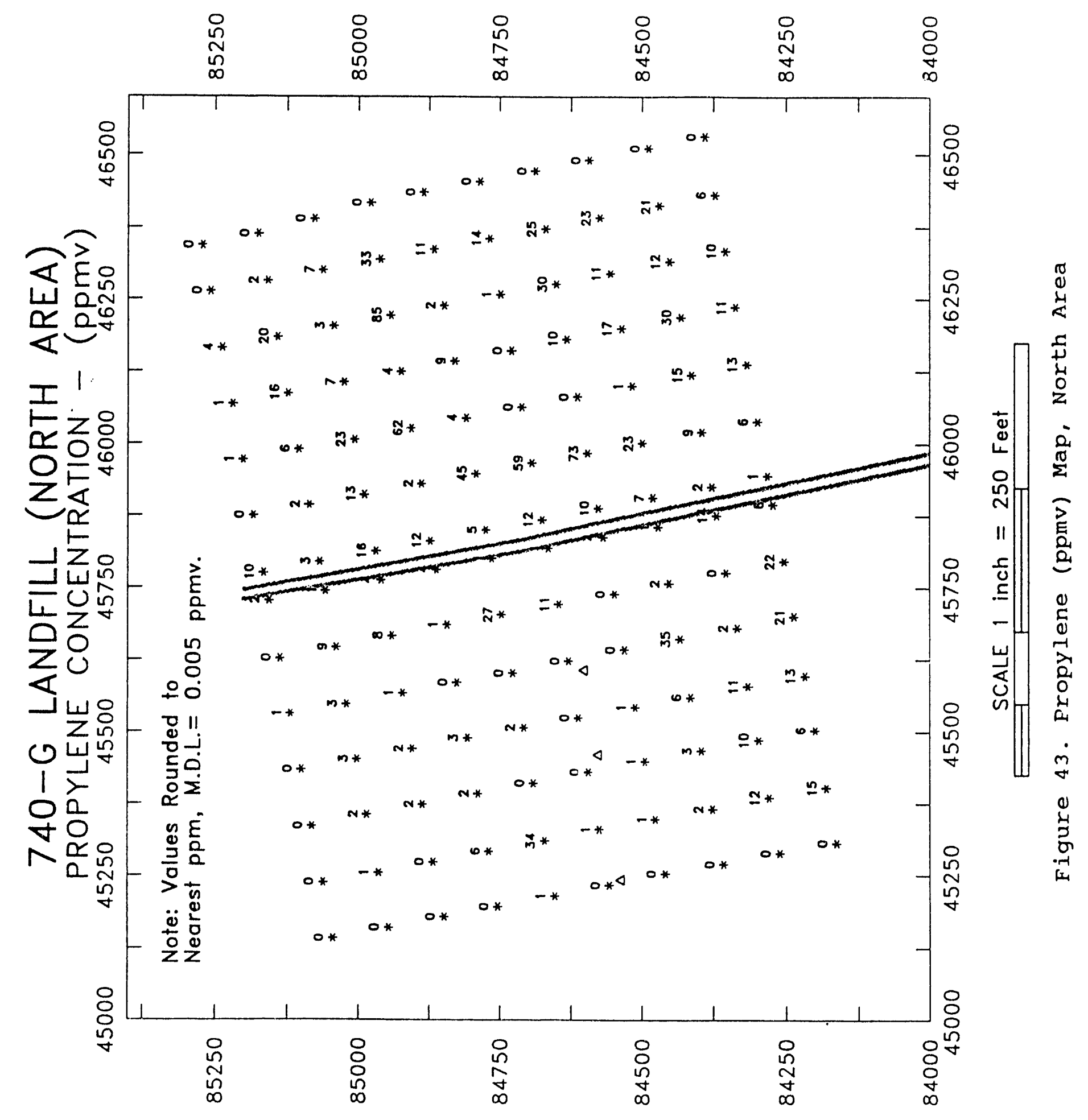




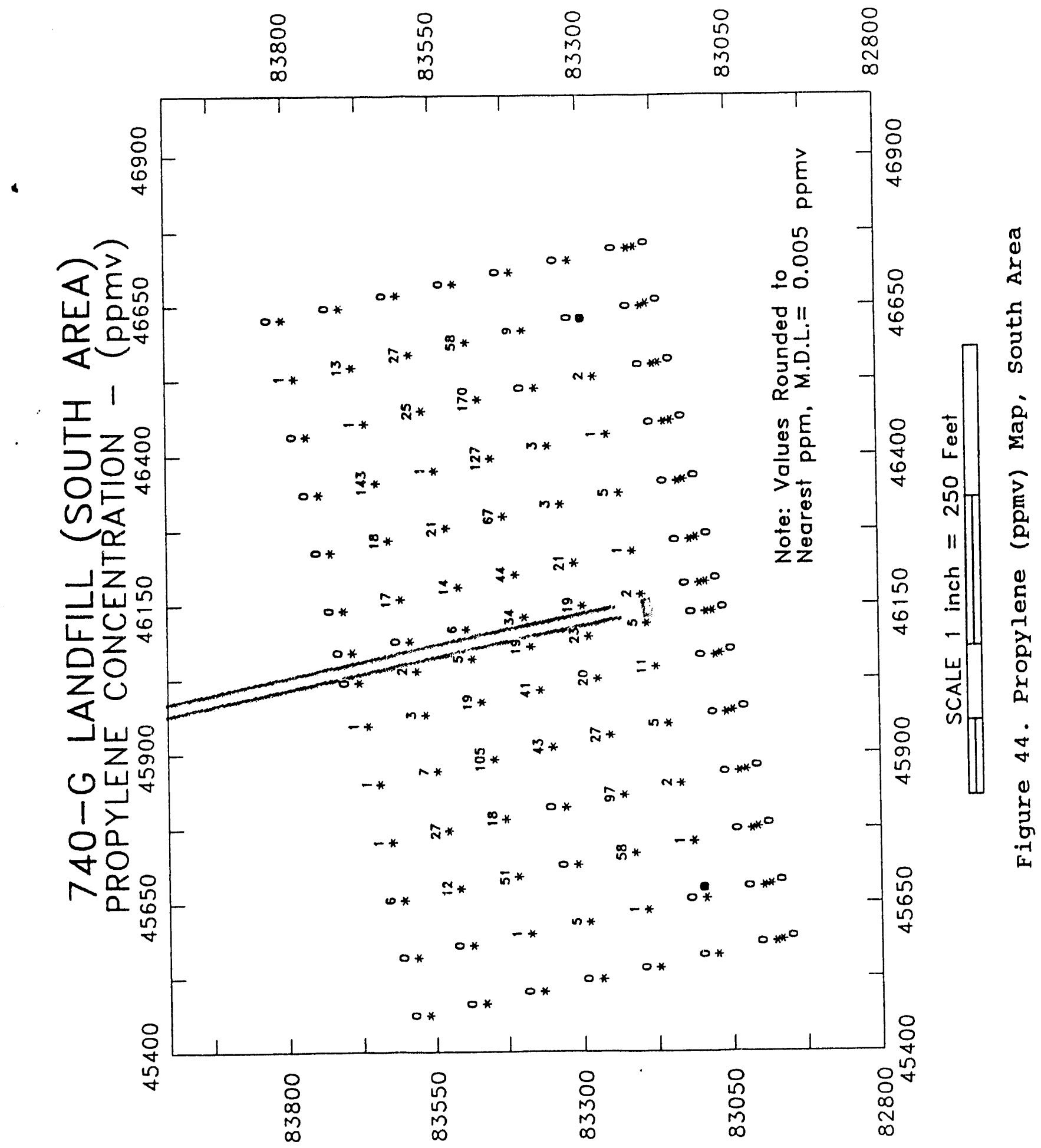




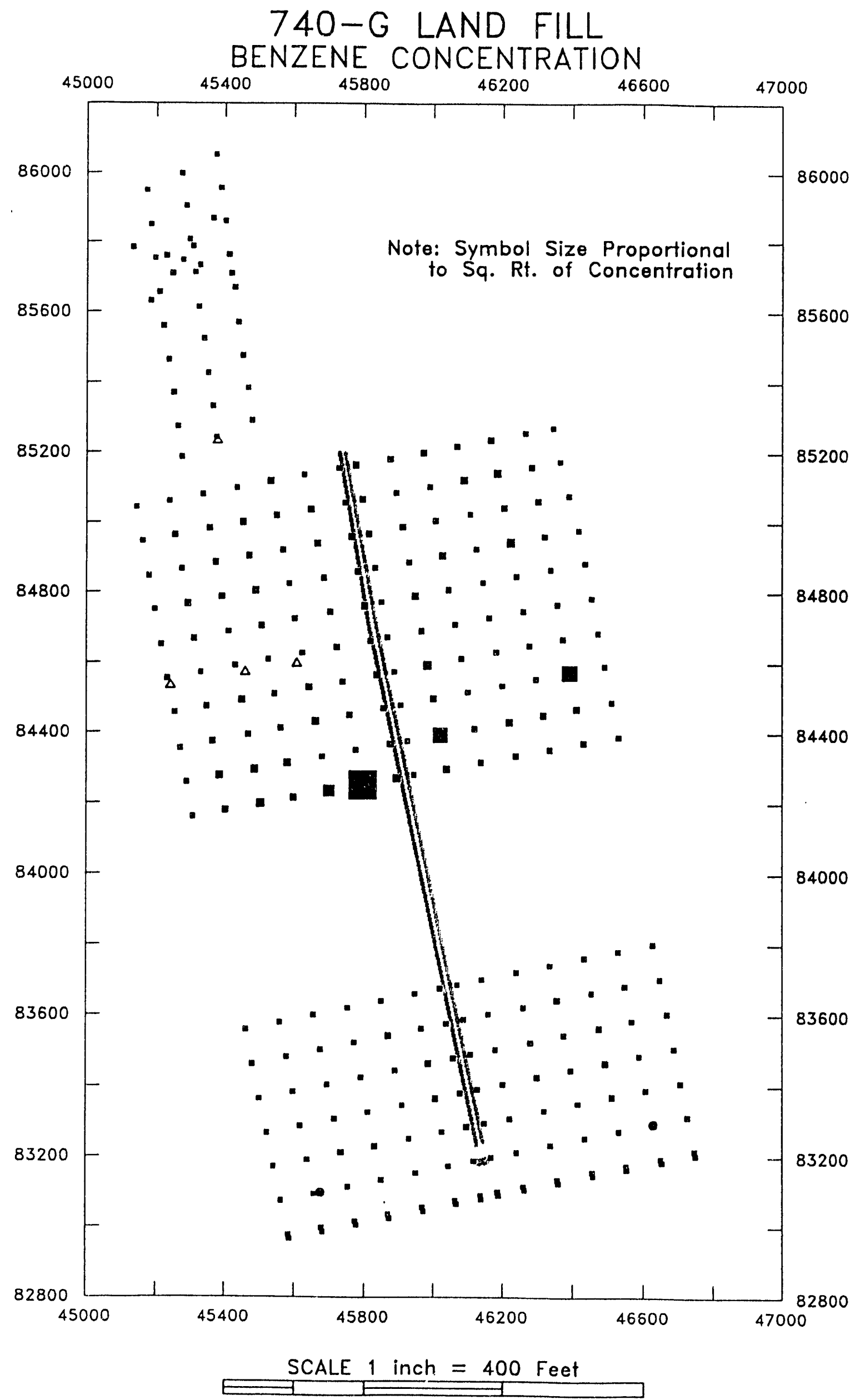

Figure 45. Benzene Symbol Map 


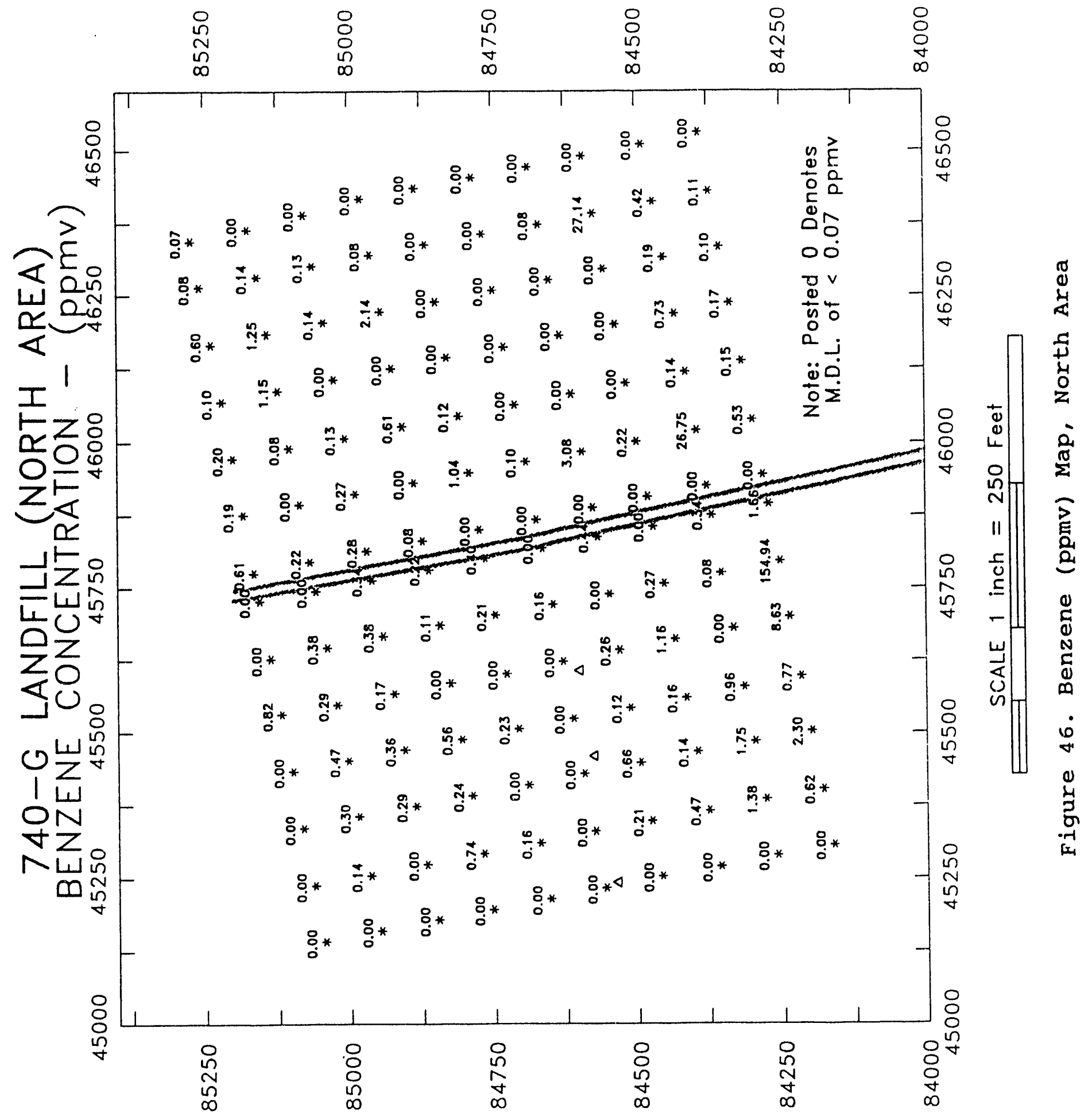




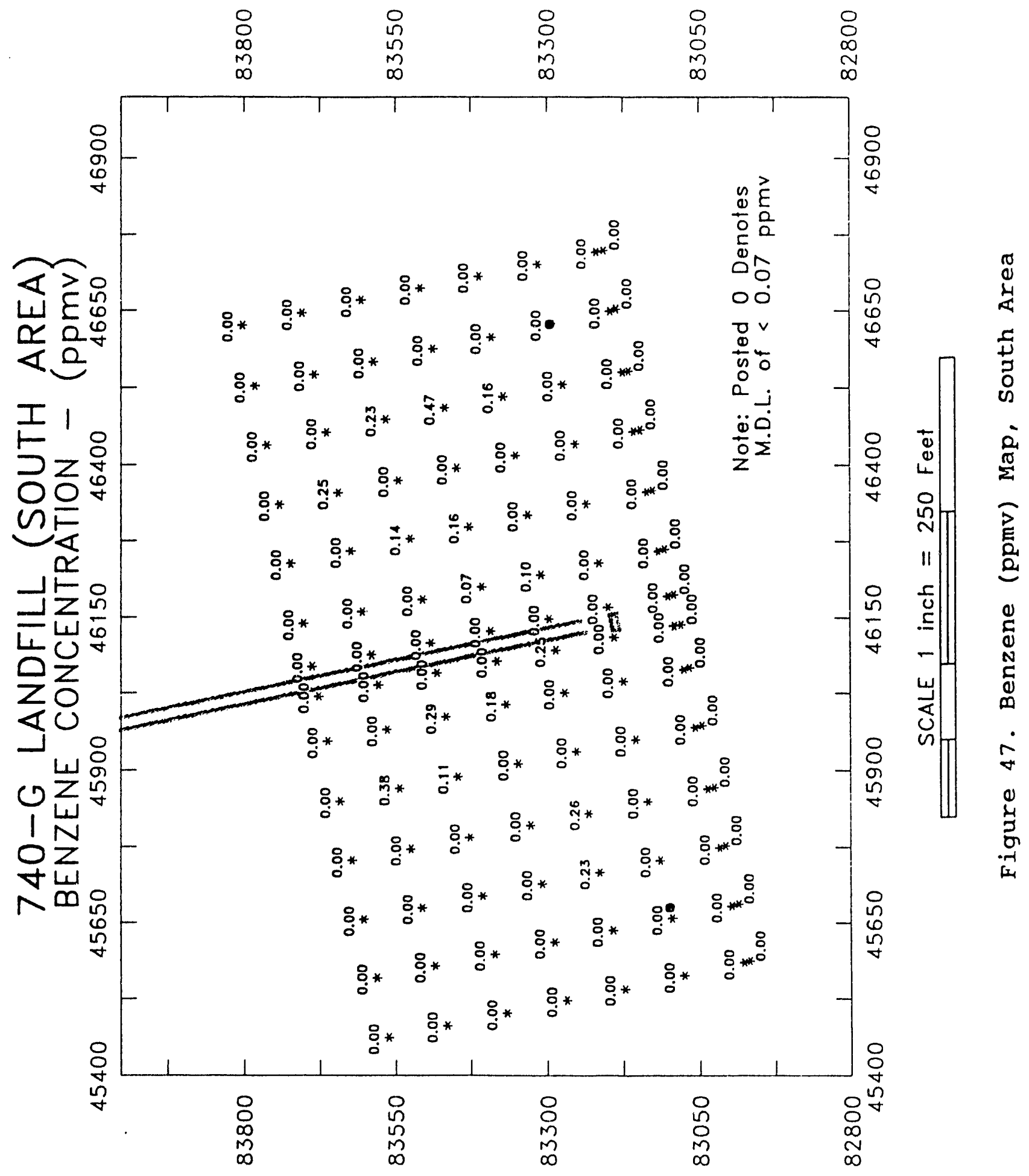




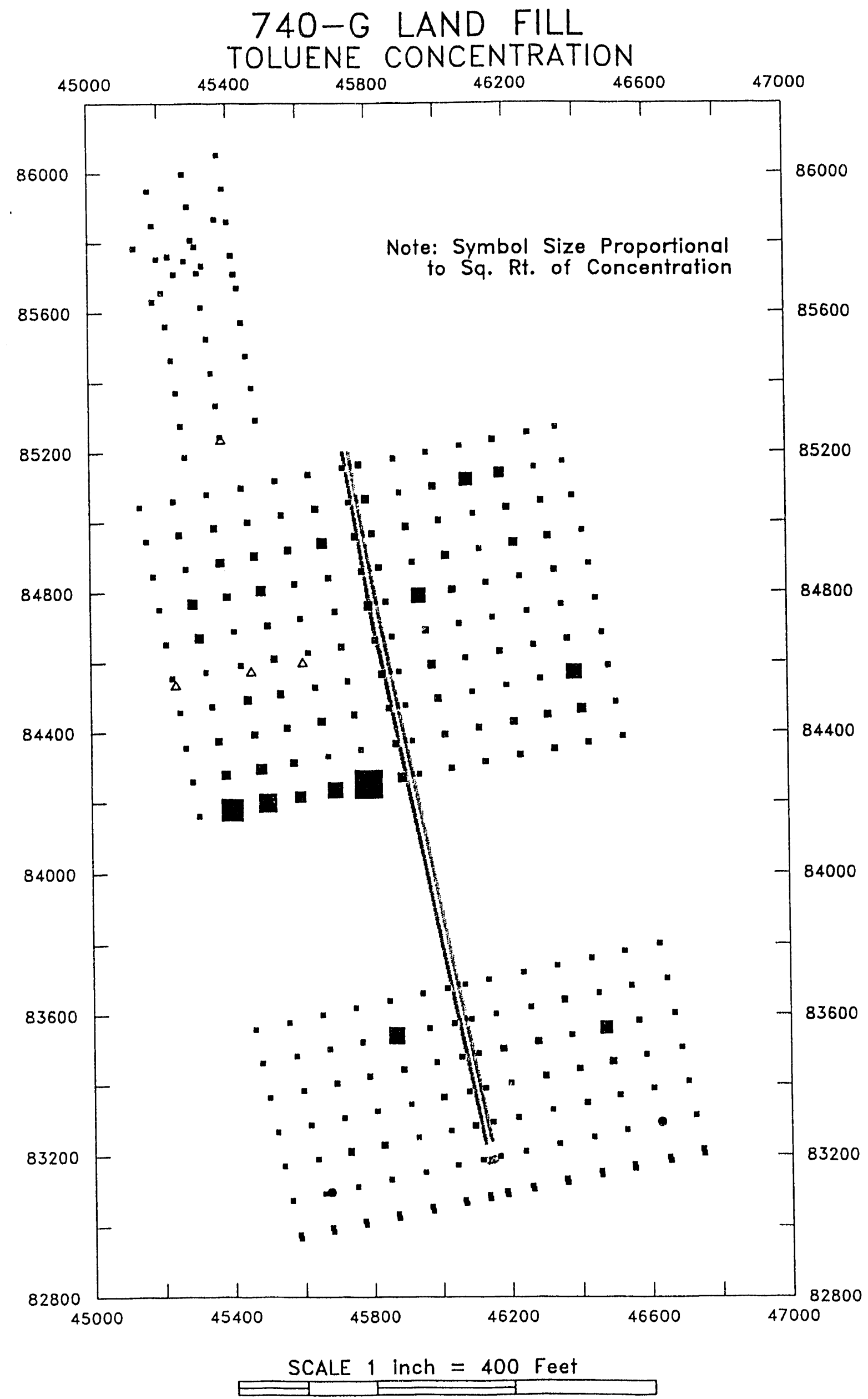

Figure 48. Toluene Symbol Map 


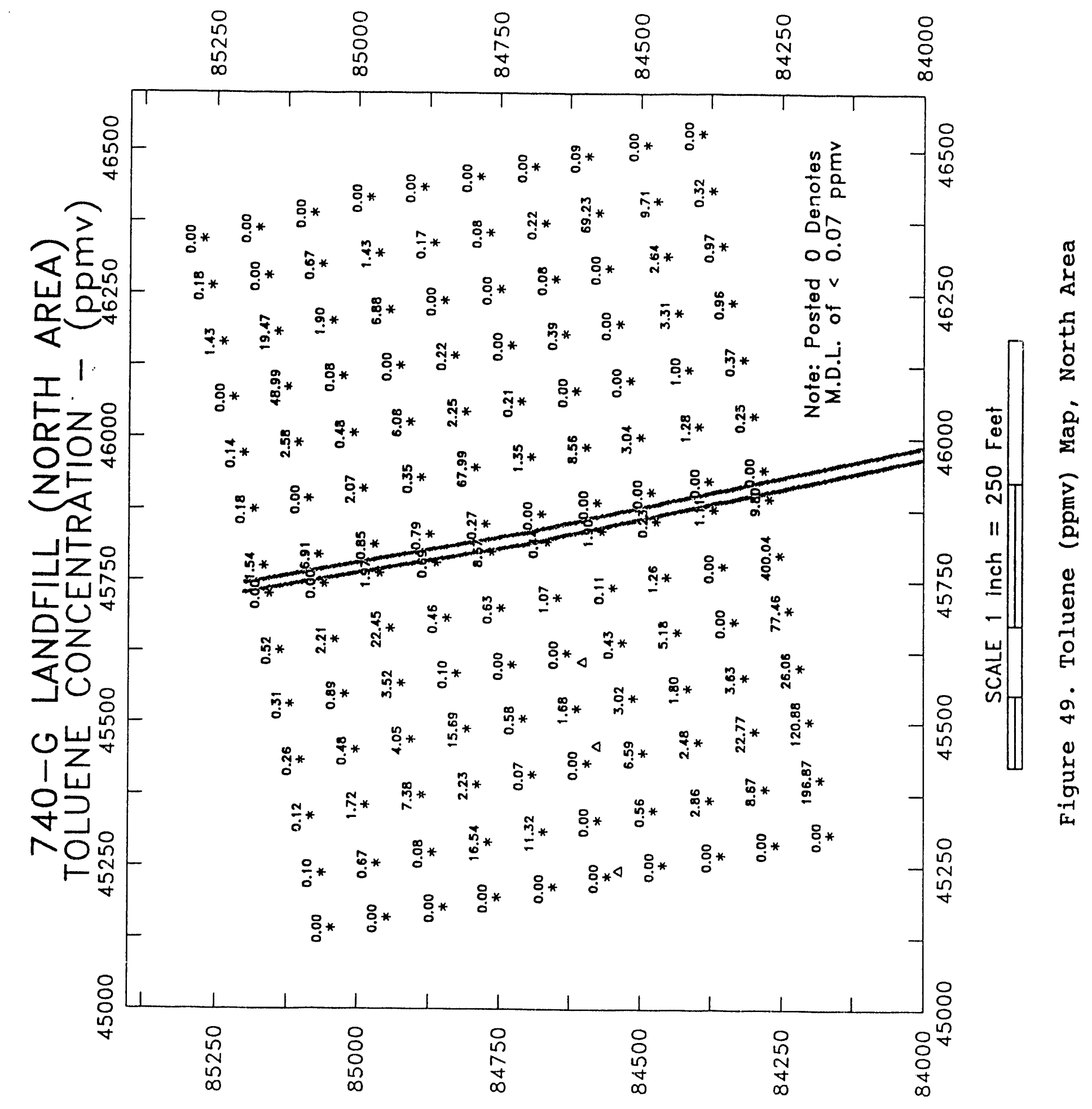




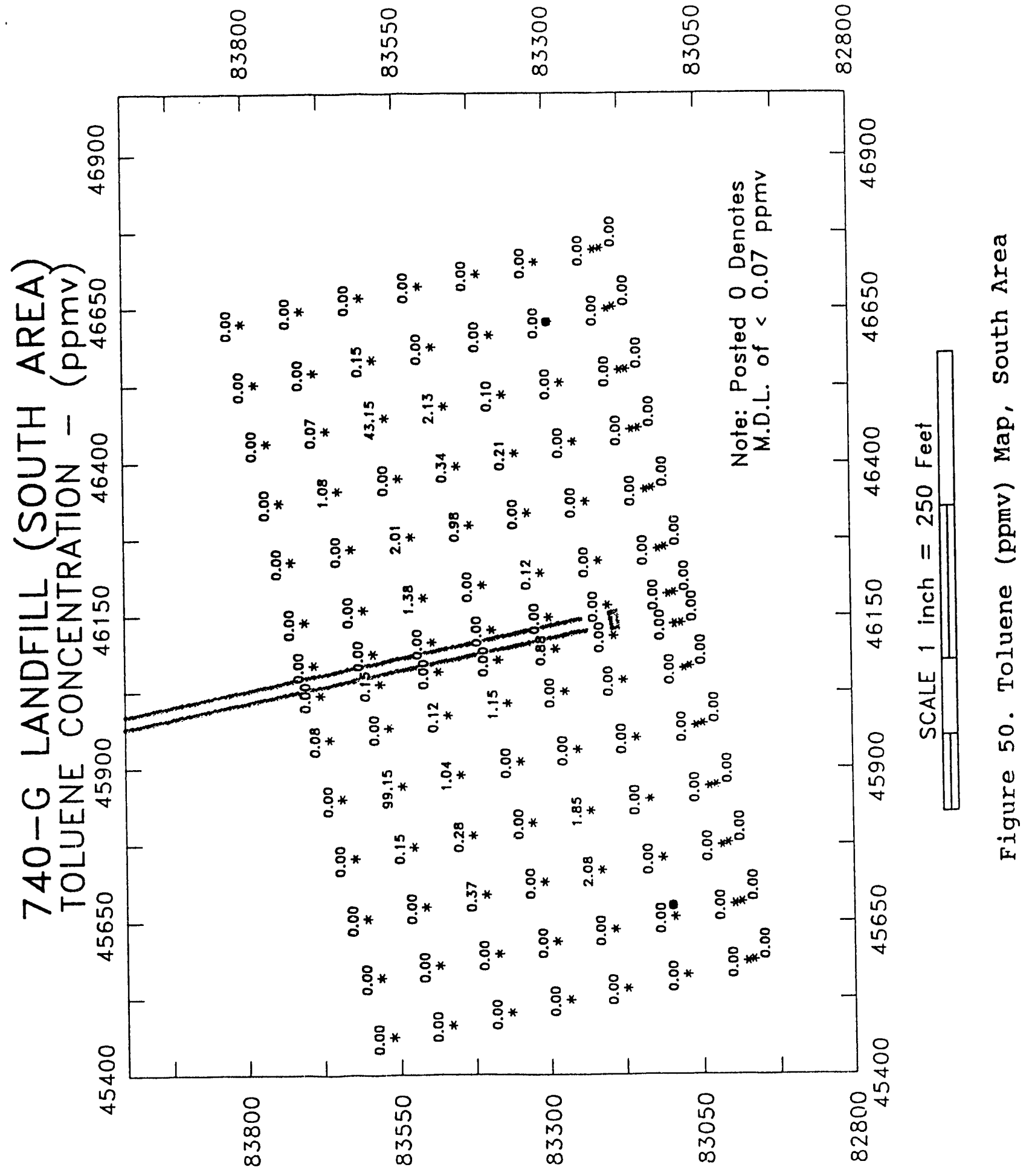




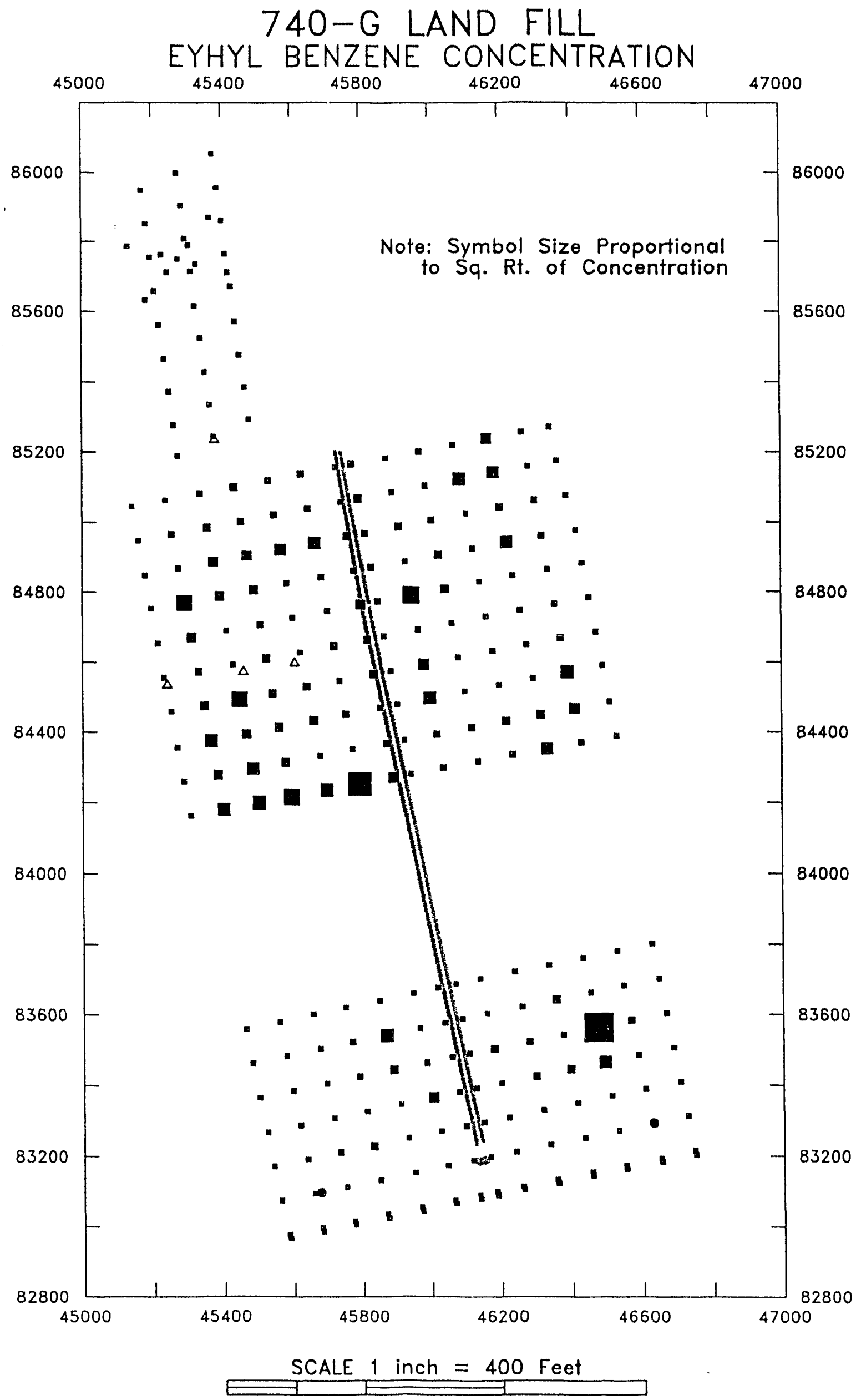

Figure 51. Ethylbenzene Symbol Map 

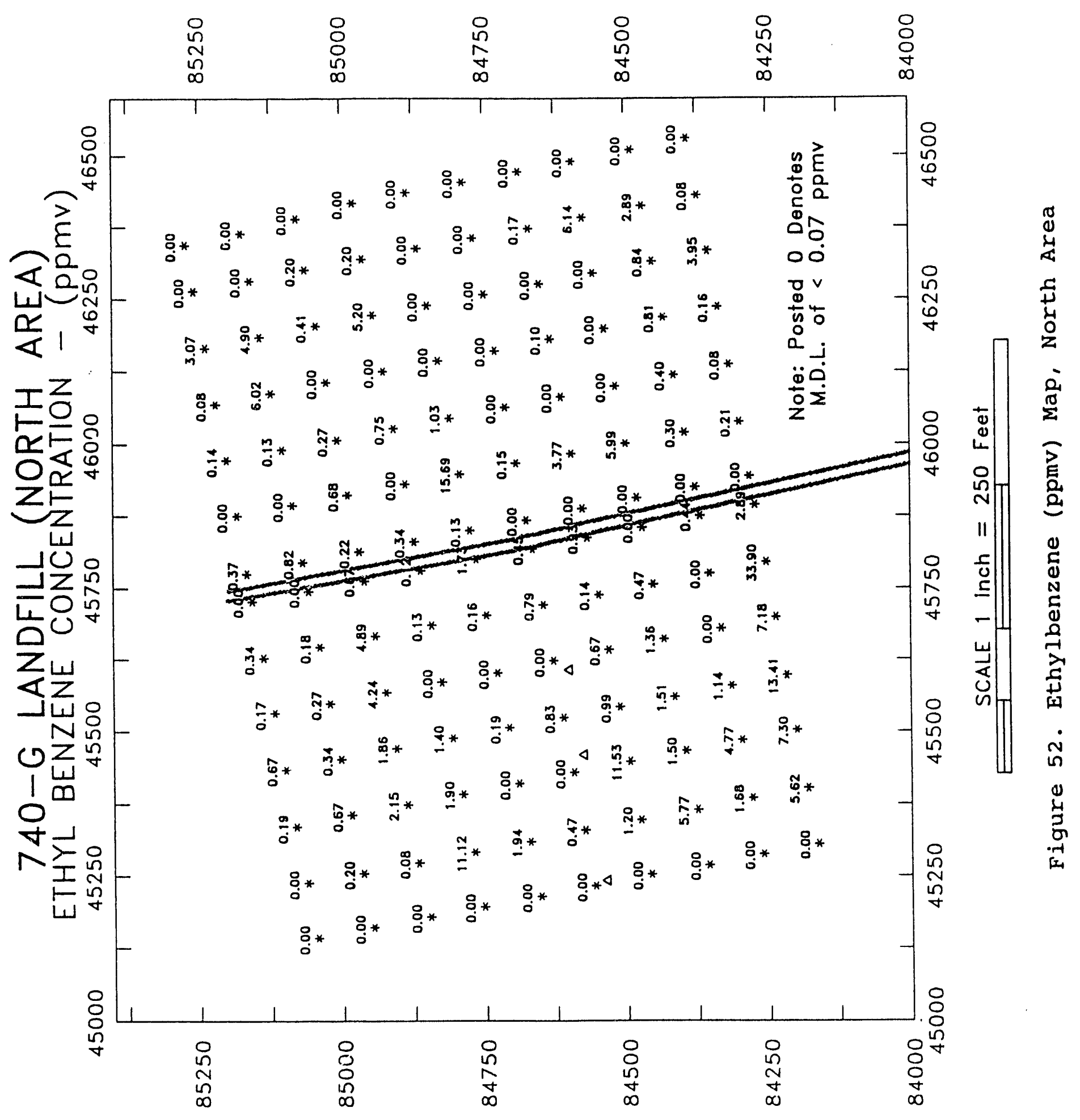


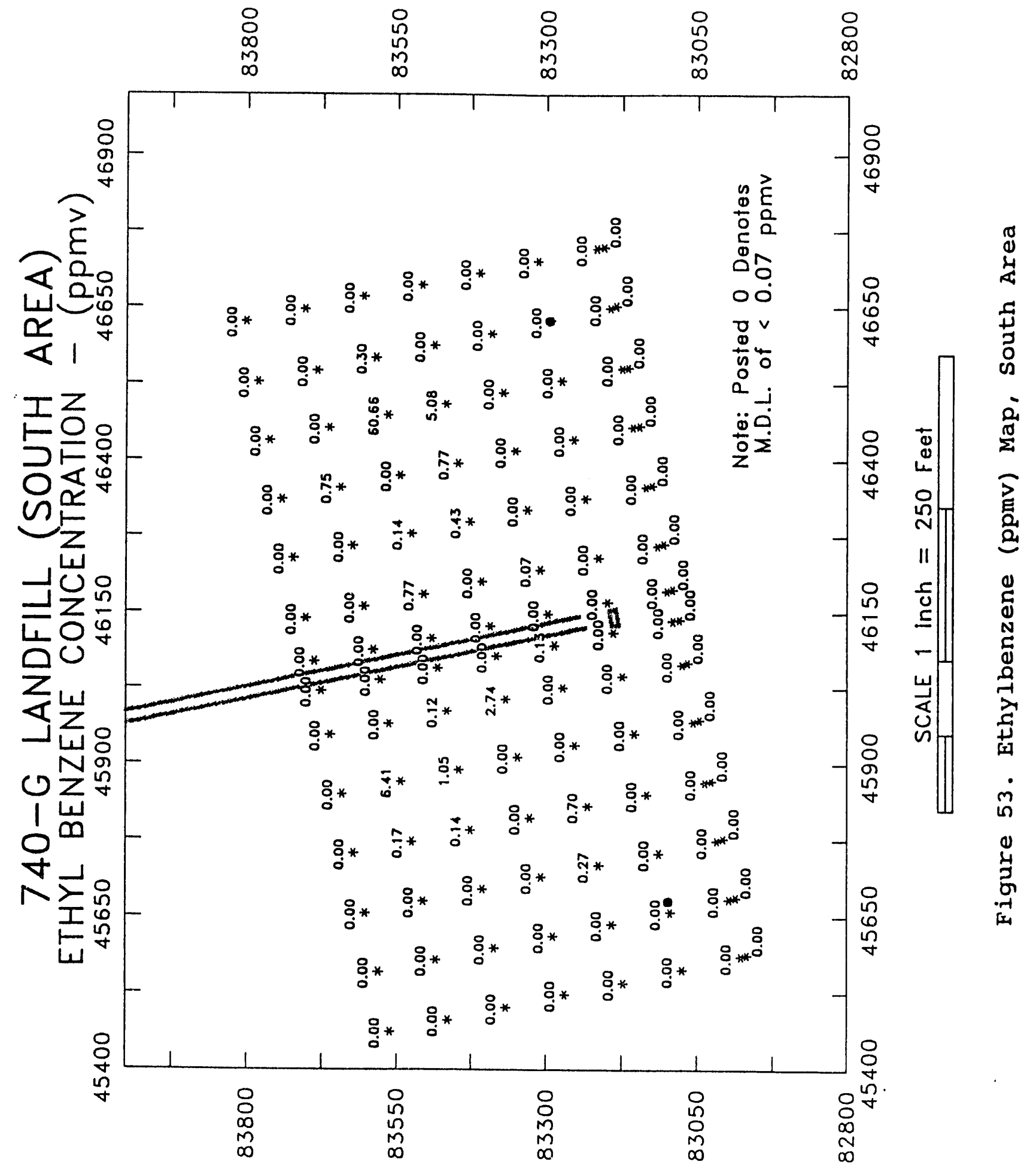




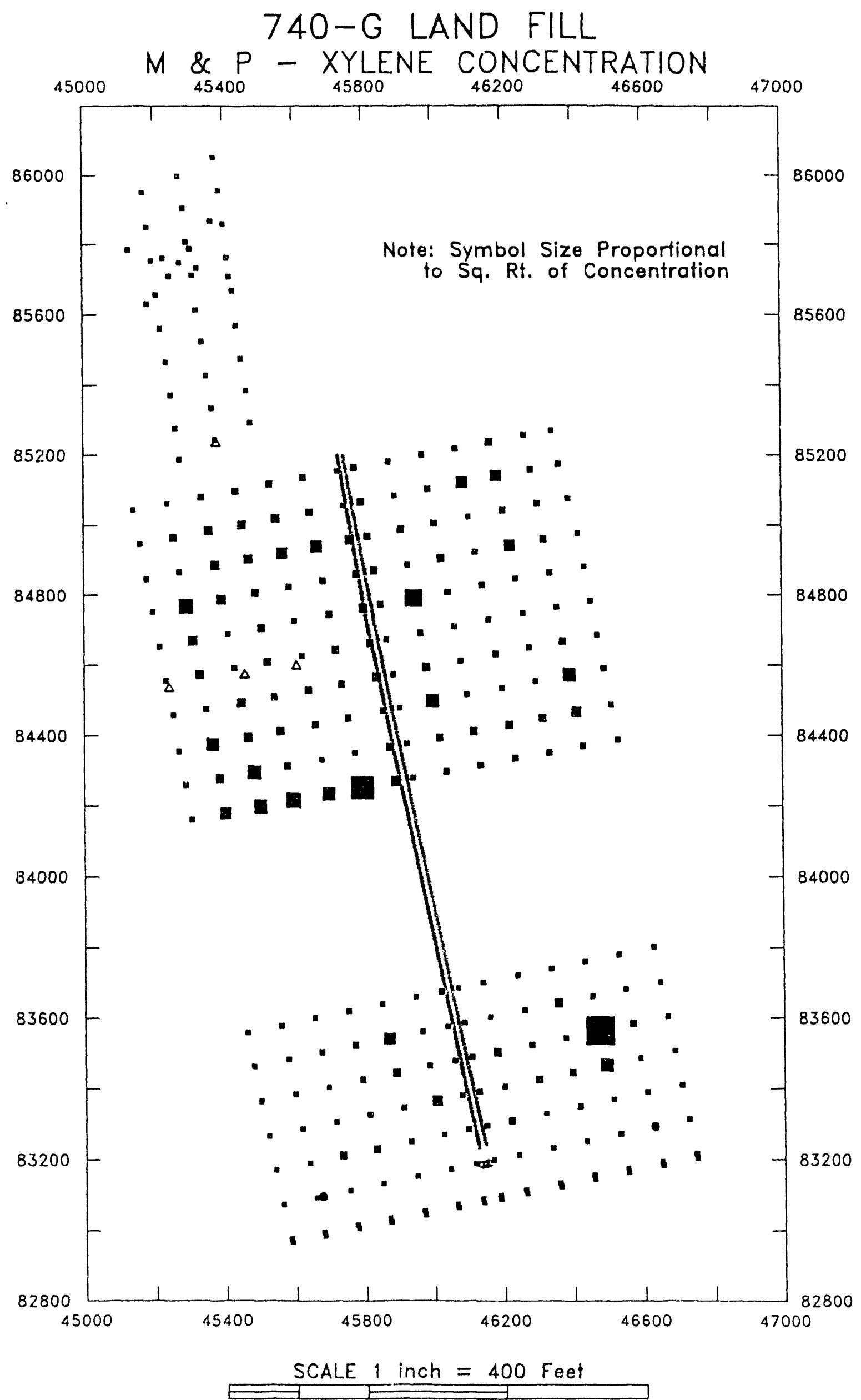

Figure 54. m- \& p-Xylene Symbol Map 


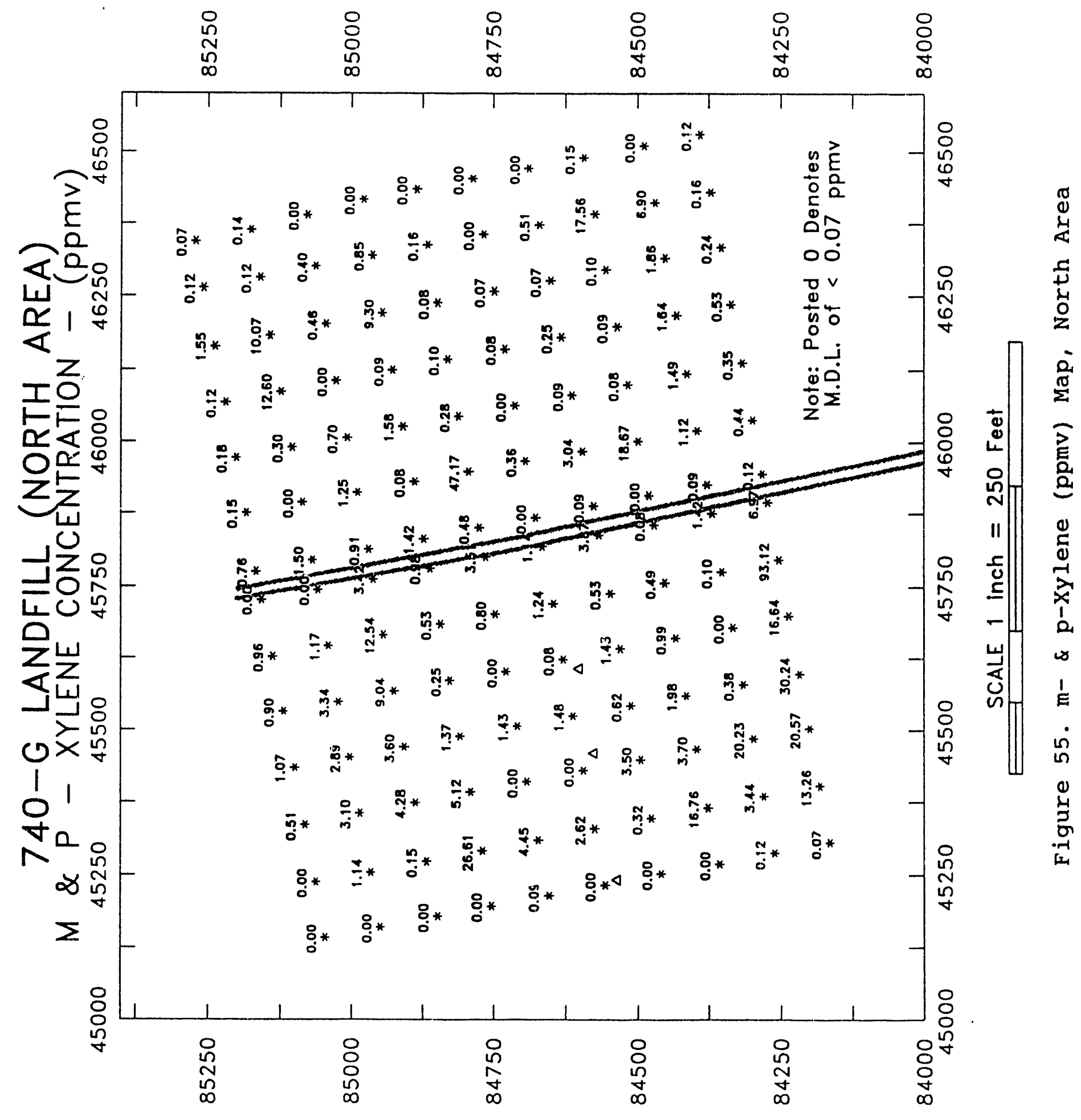




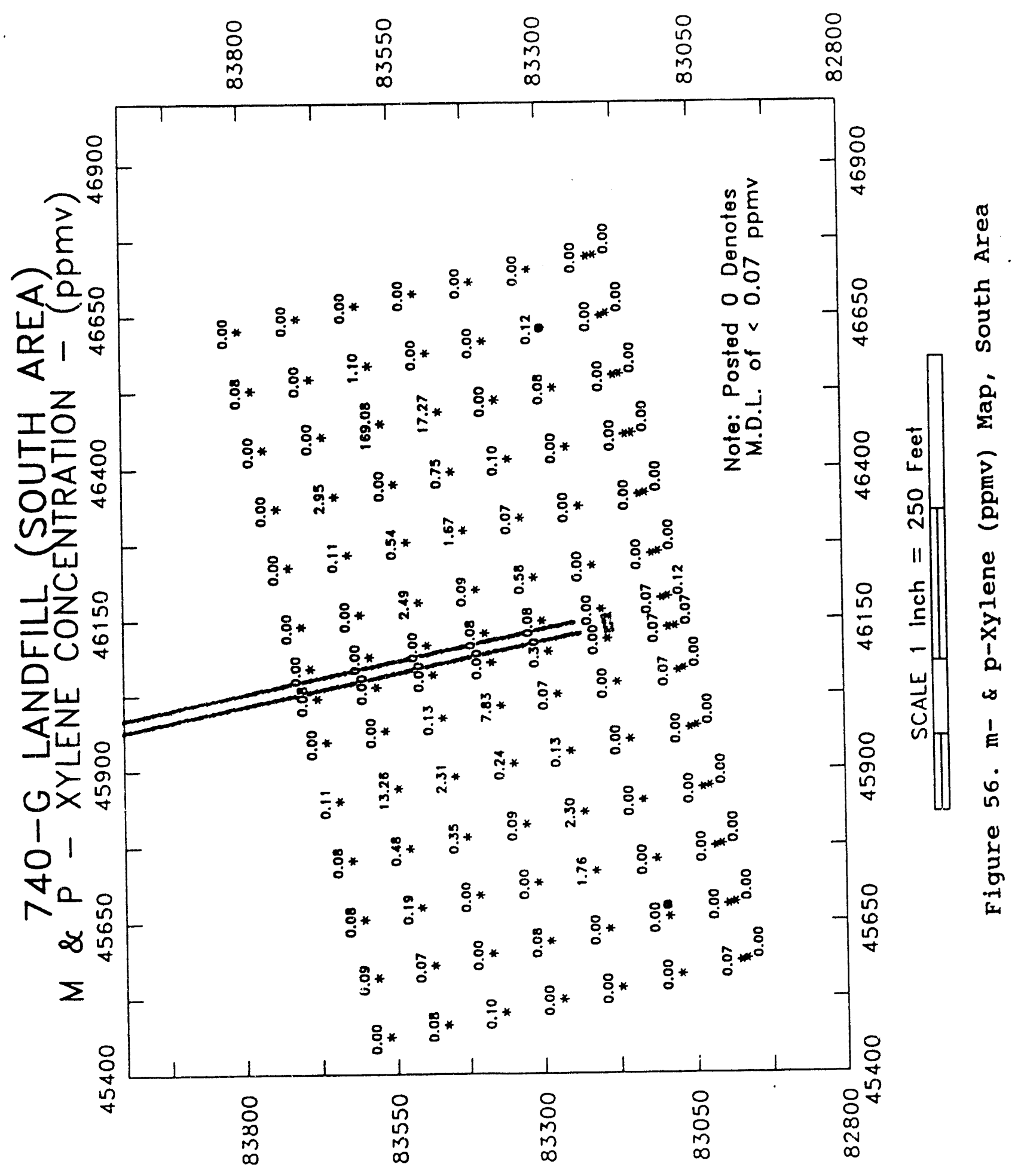




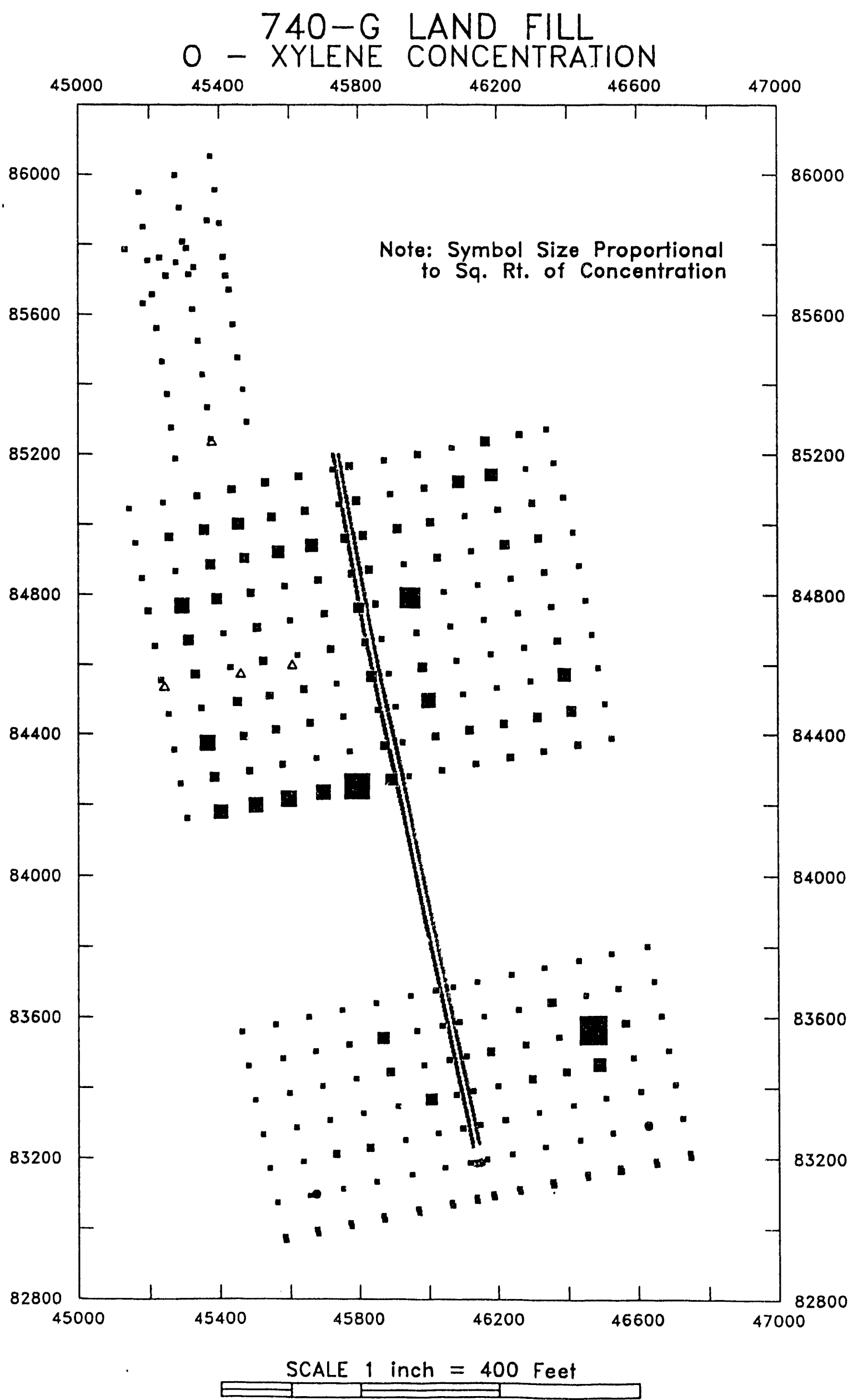

Figure 57. o-Xylene symbol Map 


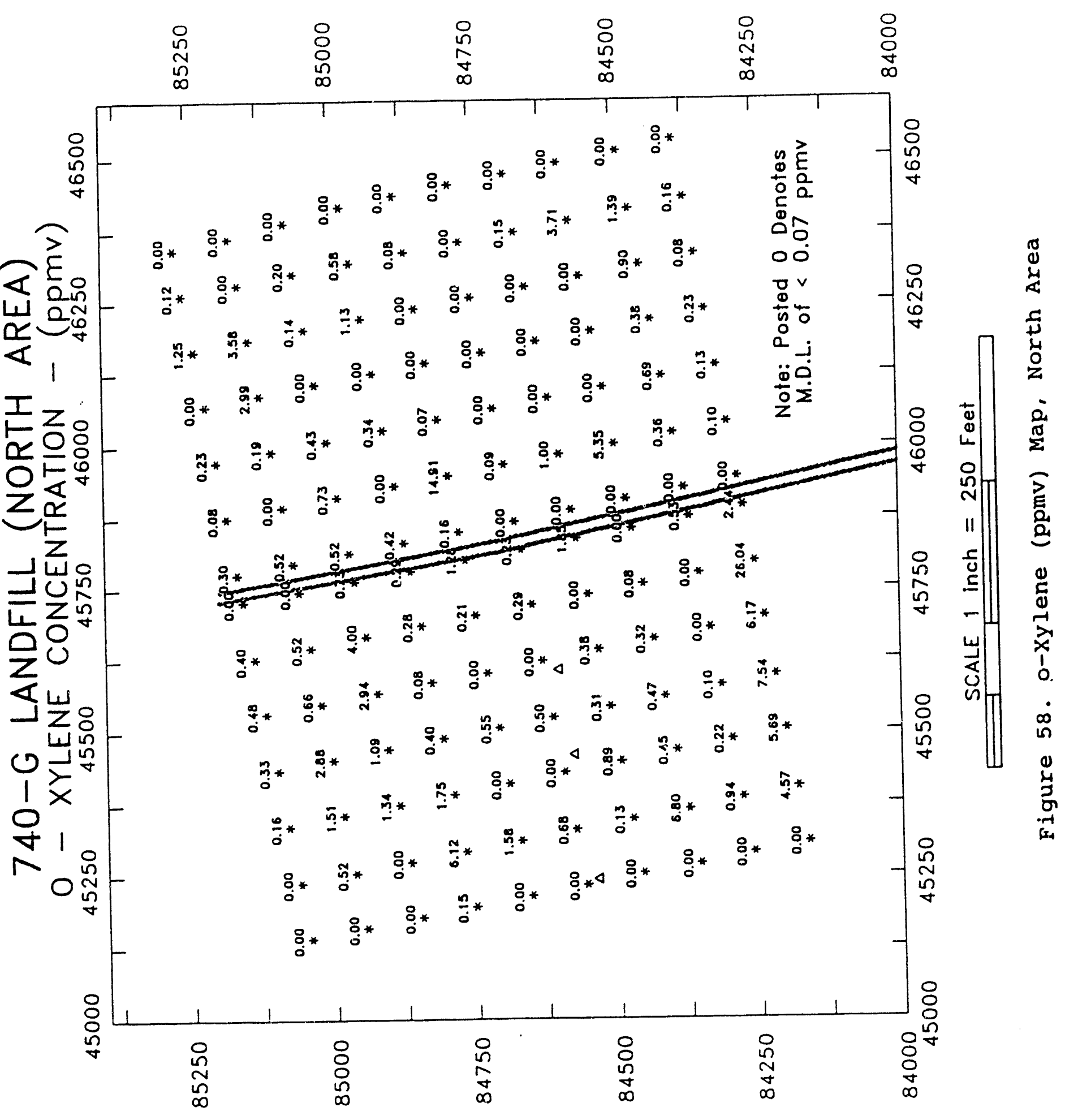




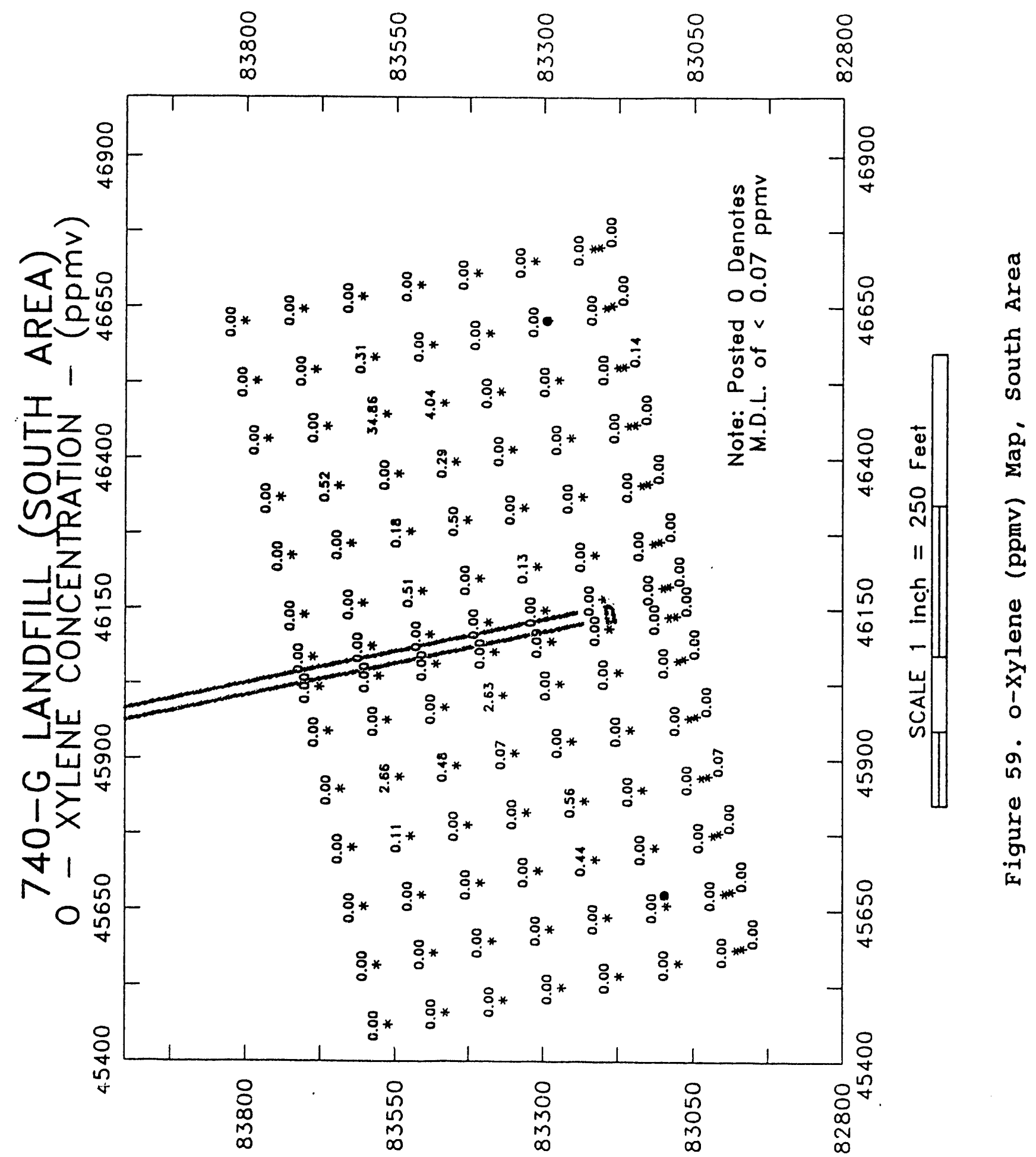




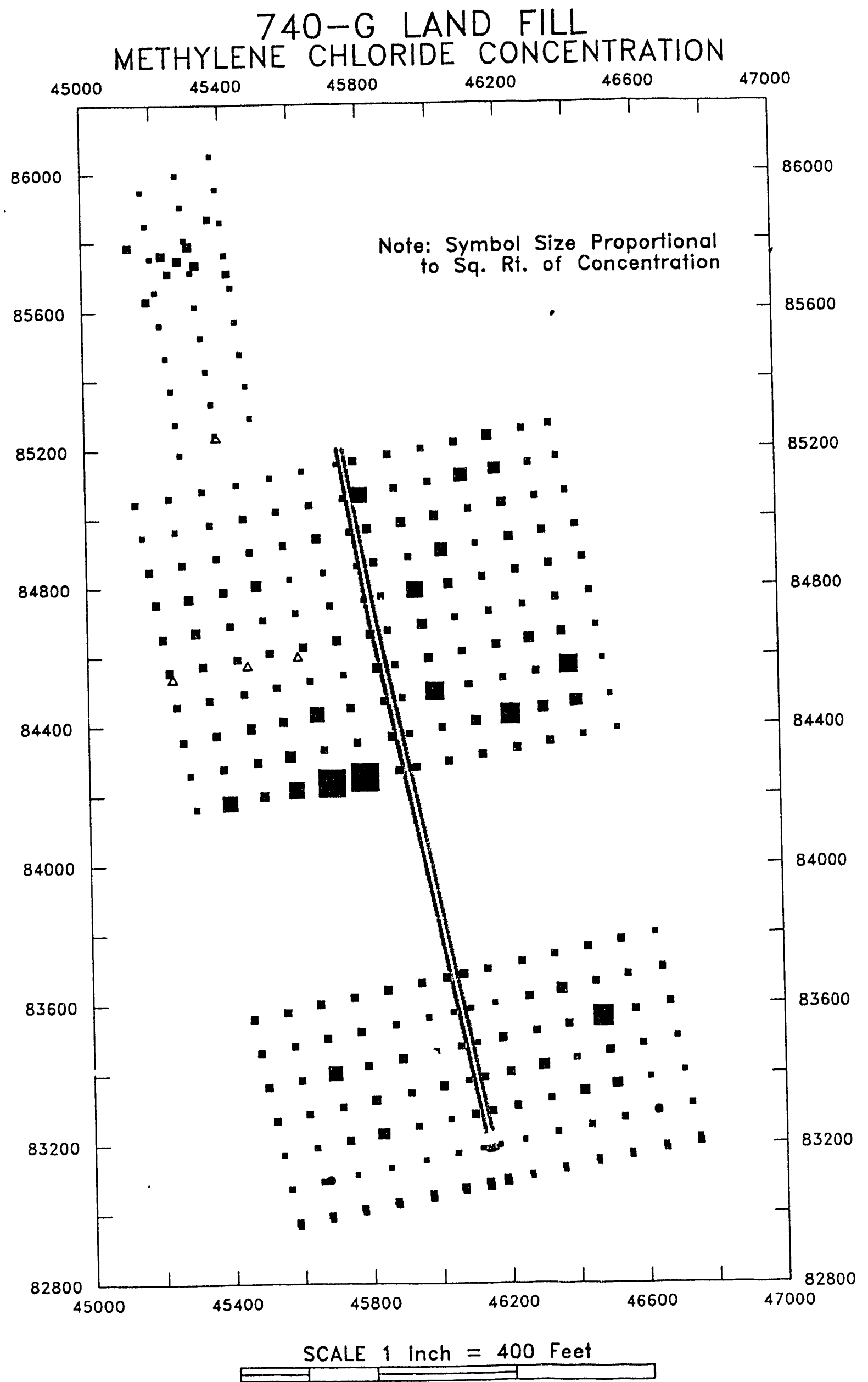

Figure 60. Methylene Chloride Symbol Map 


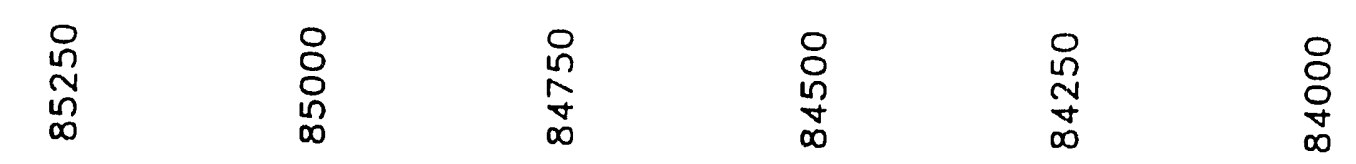

${ }^{2}+1,1+1,+1,+1,1$

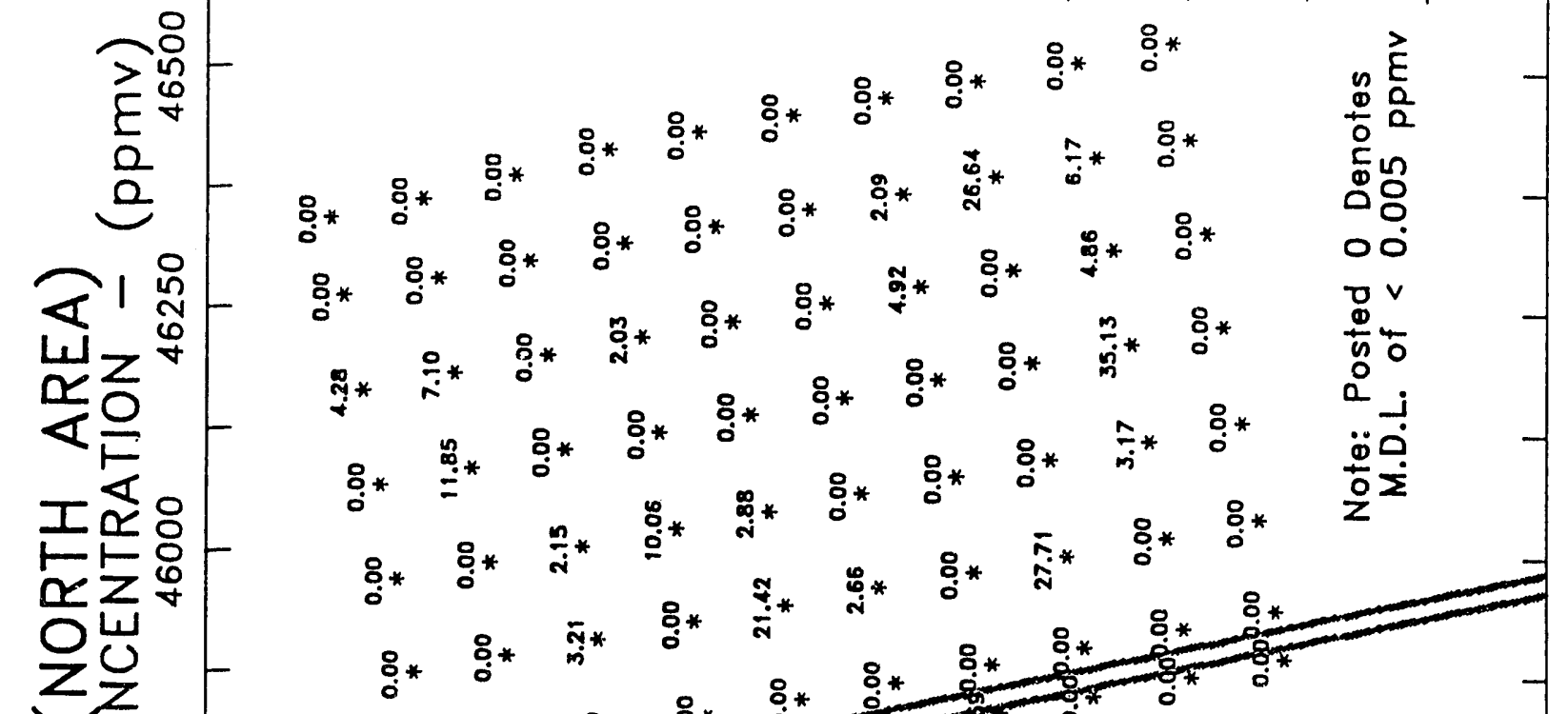

(

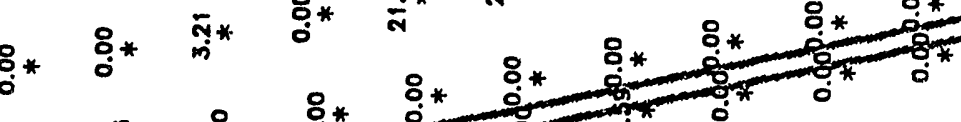

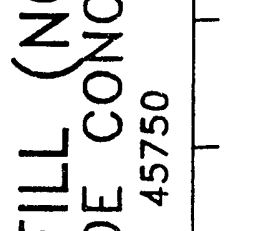

Lis

之o

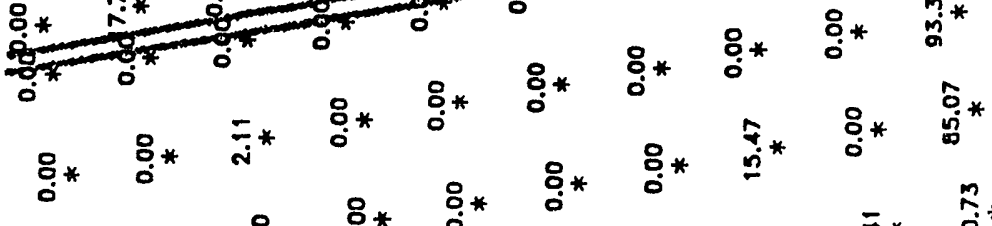

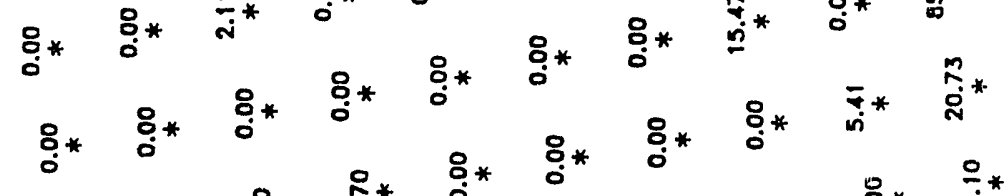

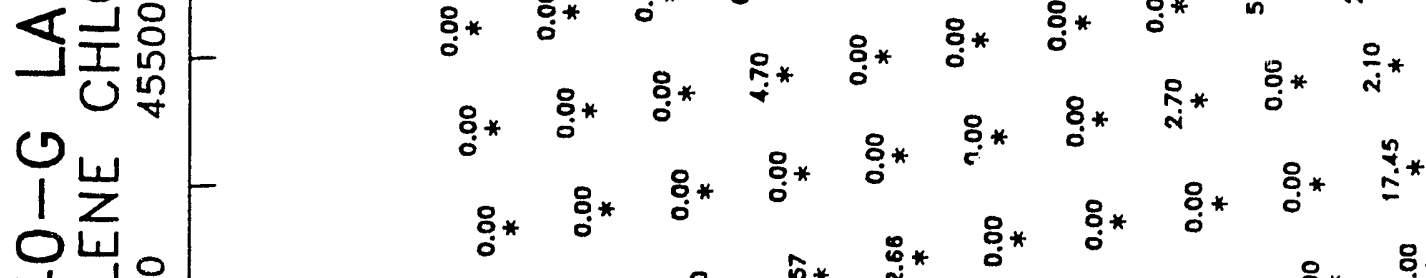

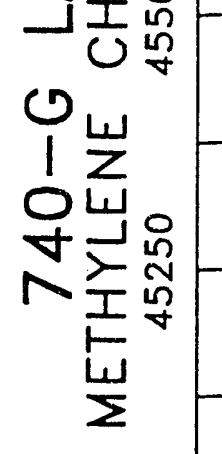

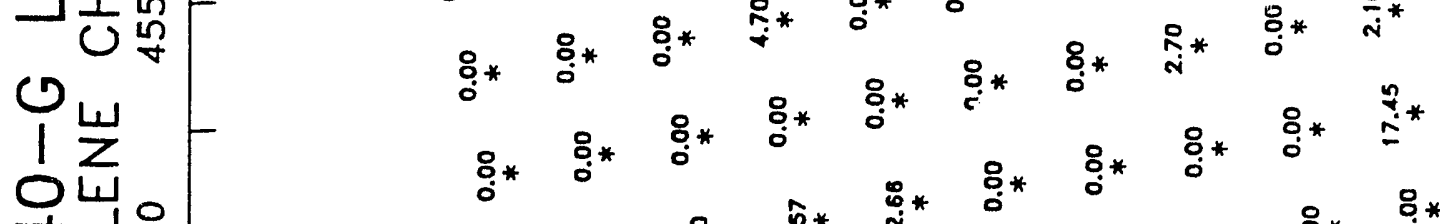

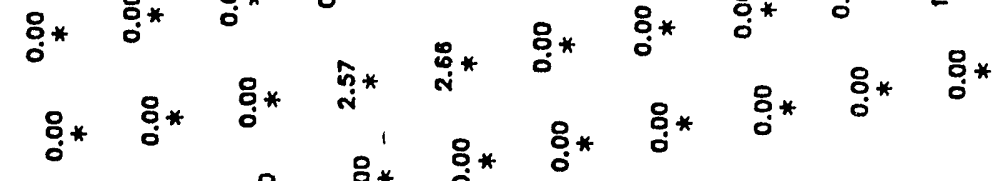

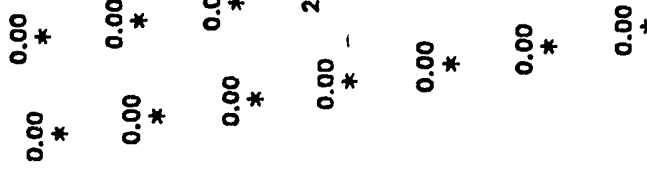

$\Psi$ I

응

ท

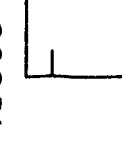




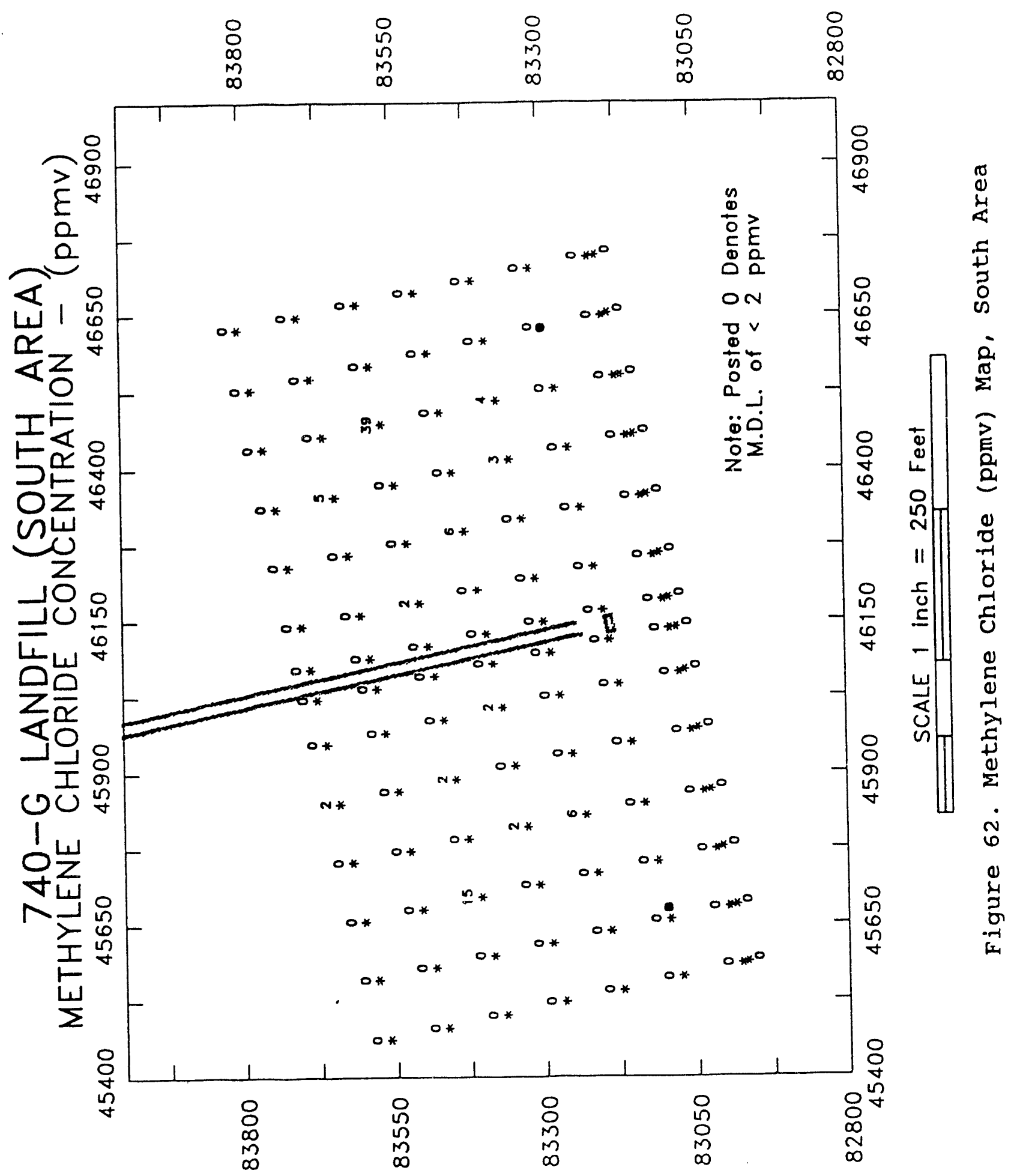




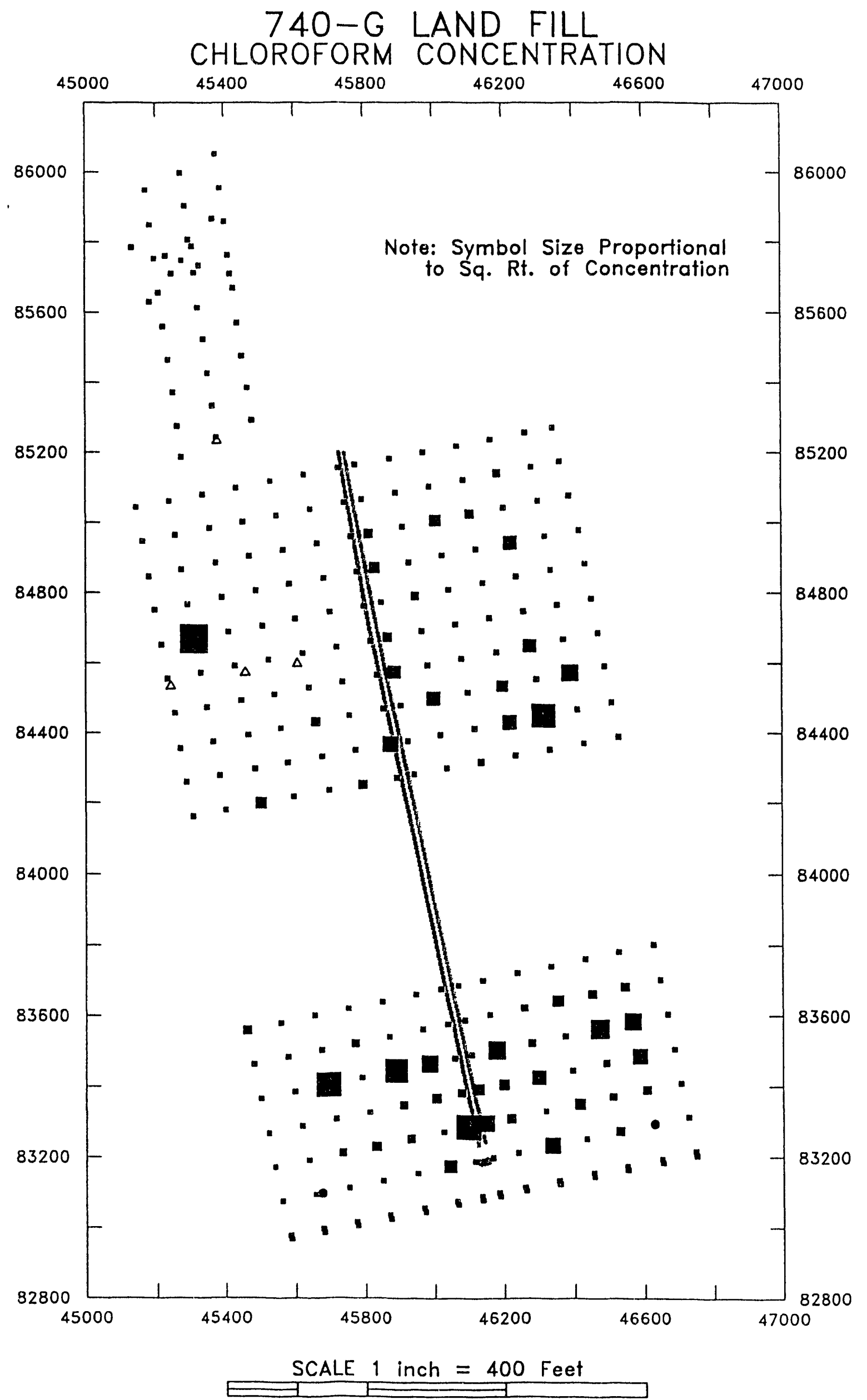

Figure 63. Chloroform Symbol Map 


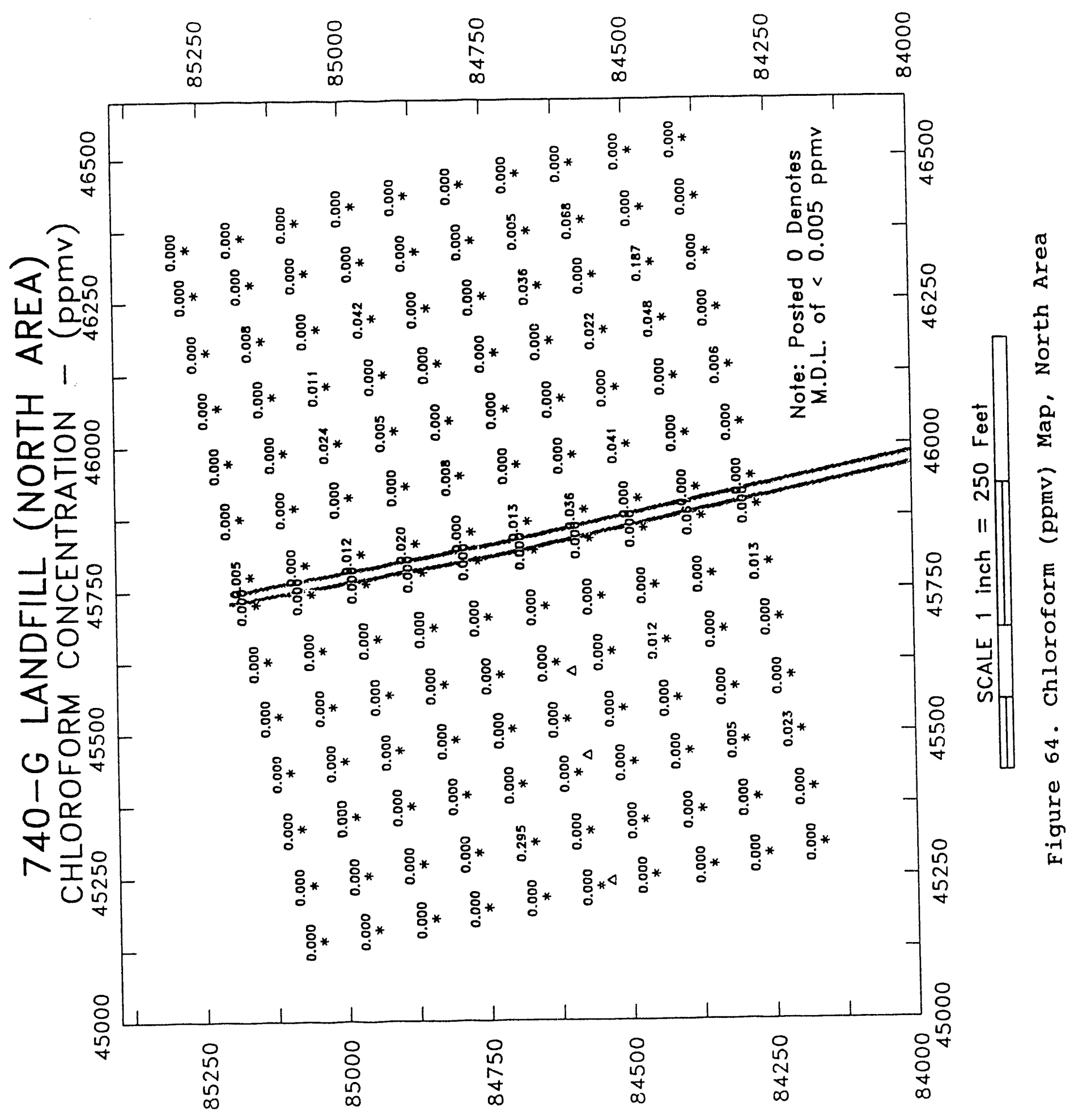




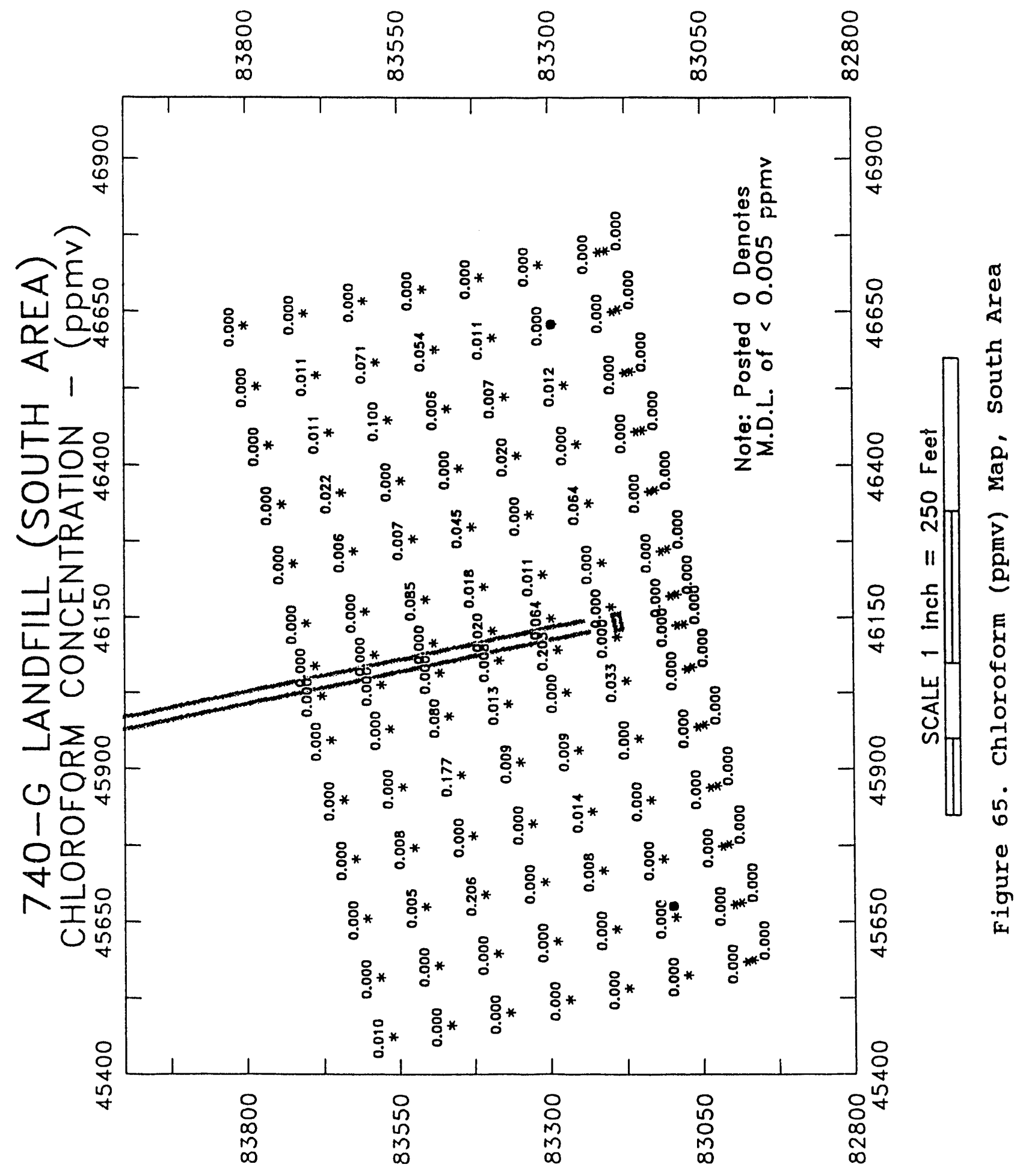




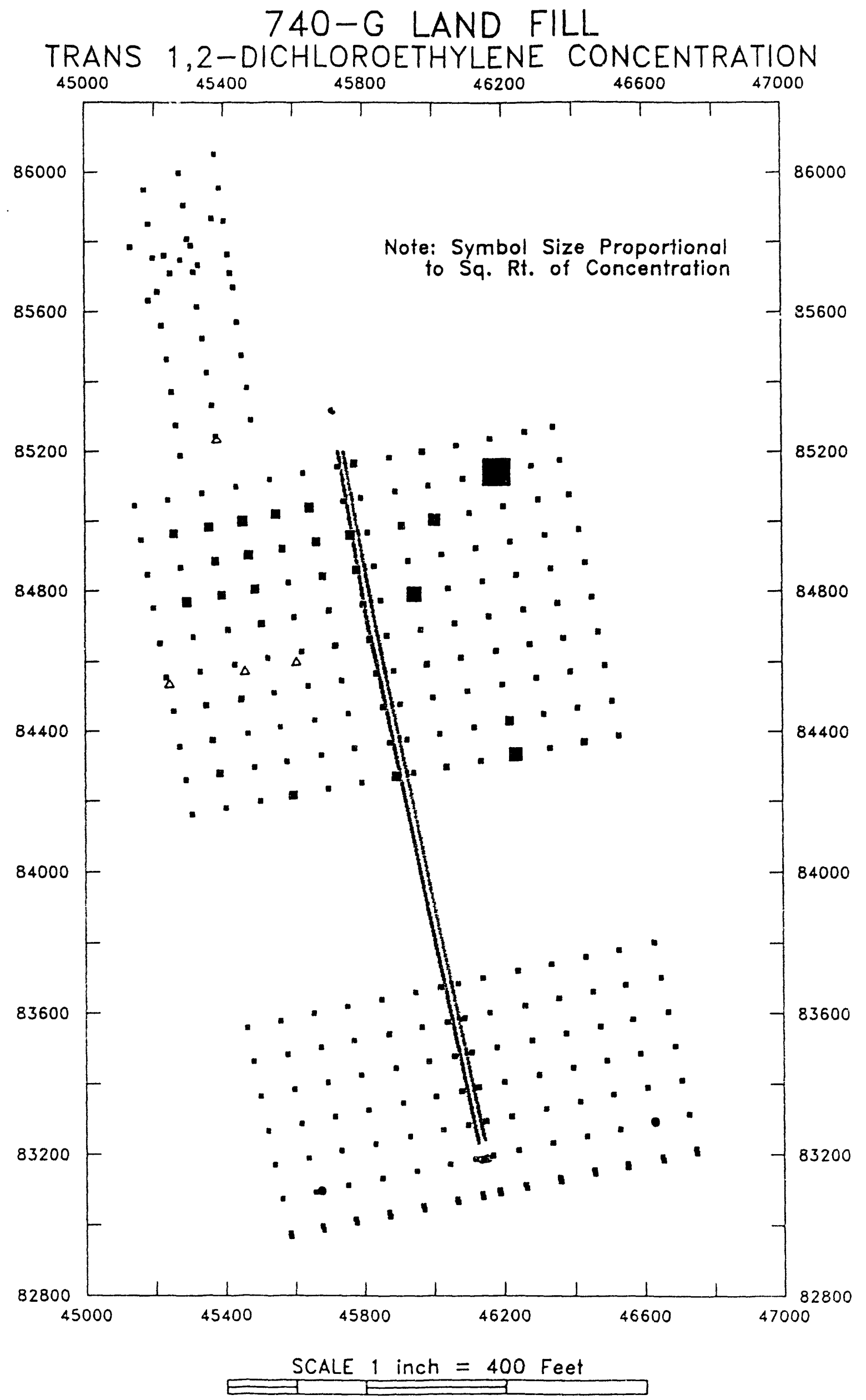

Figure 66. trans 1,2-Dichloroethylene symbol Map 


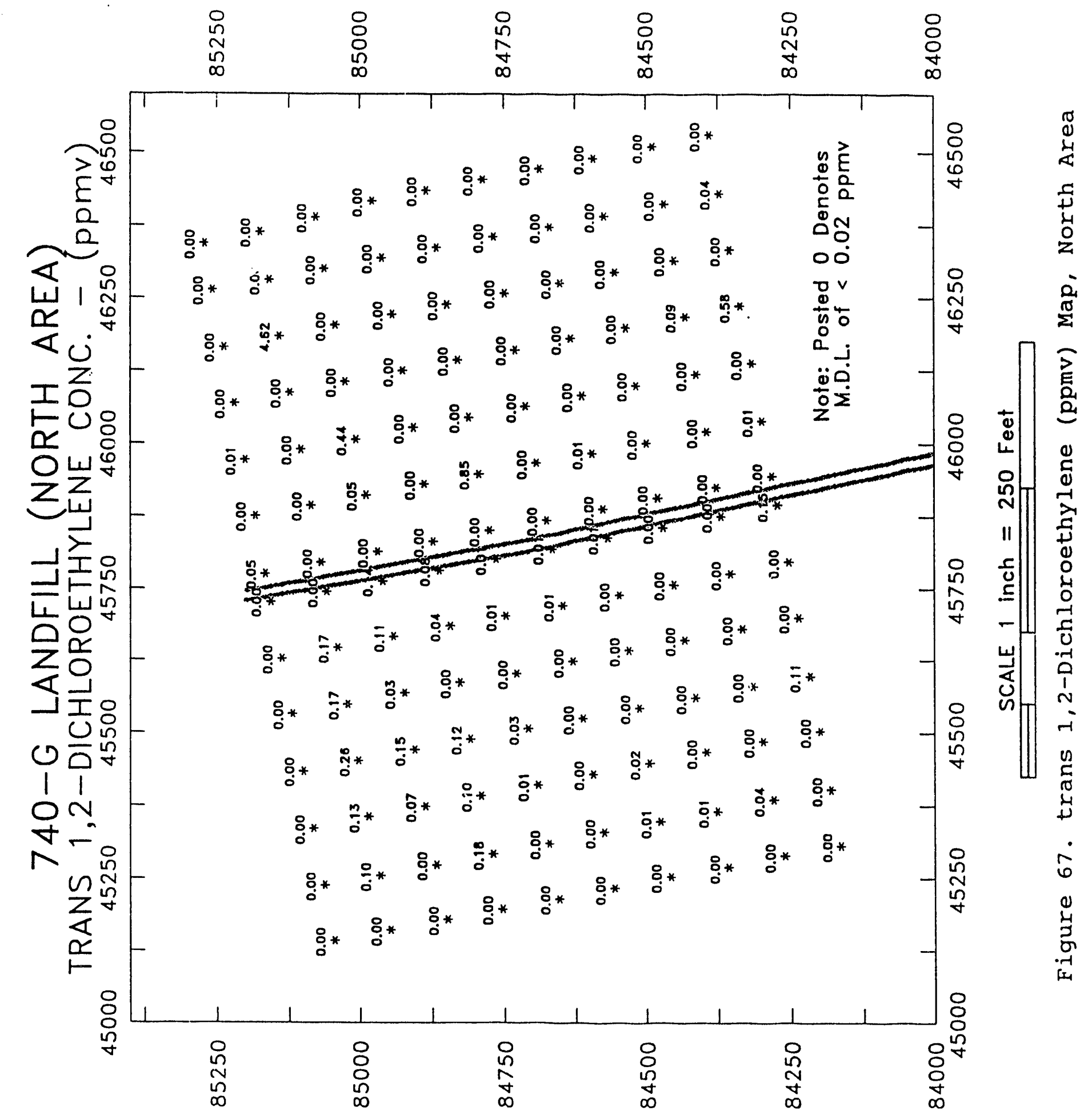




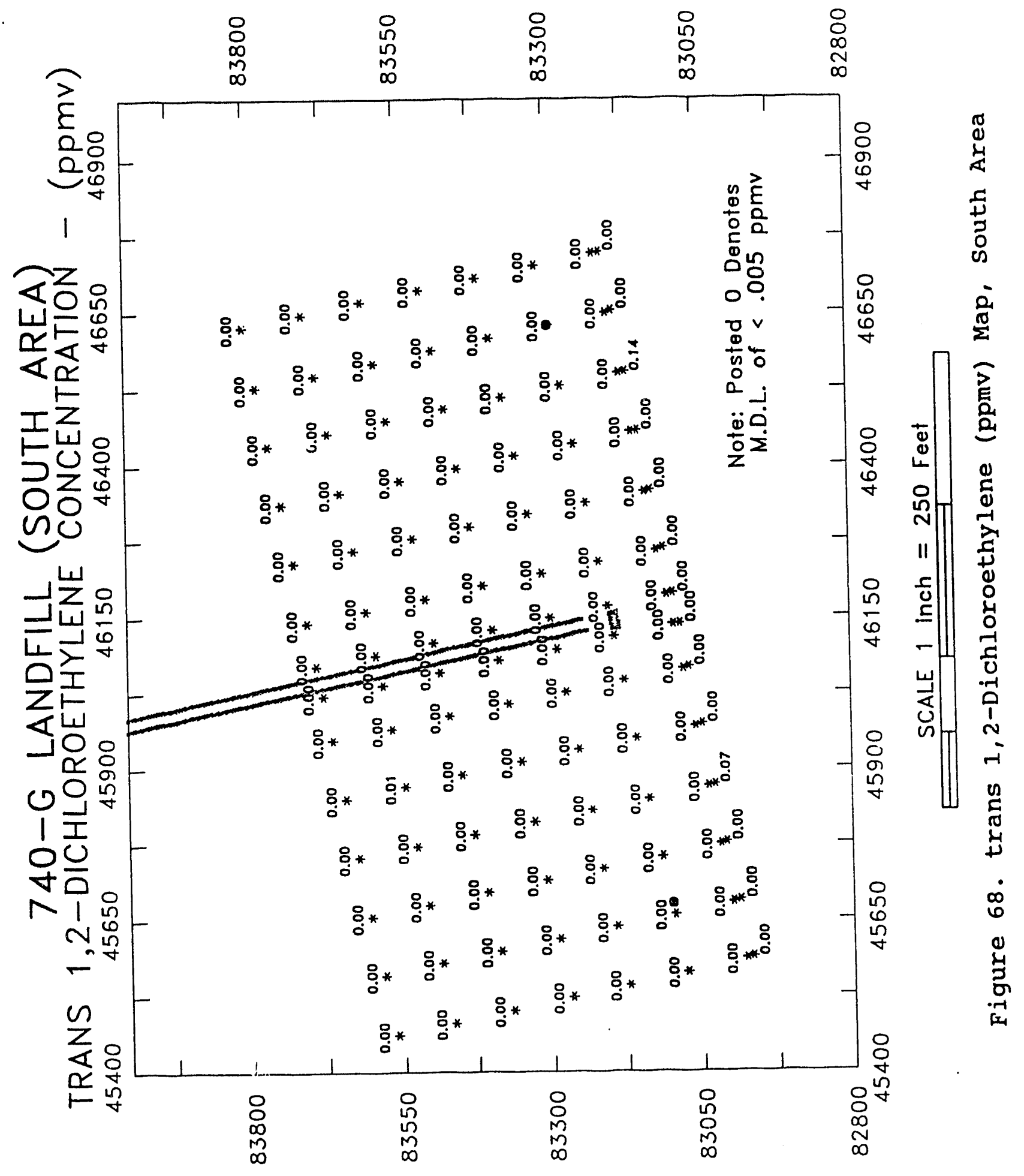




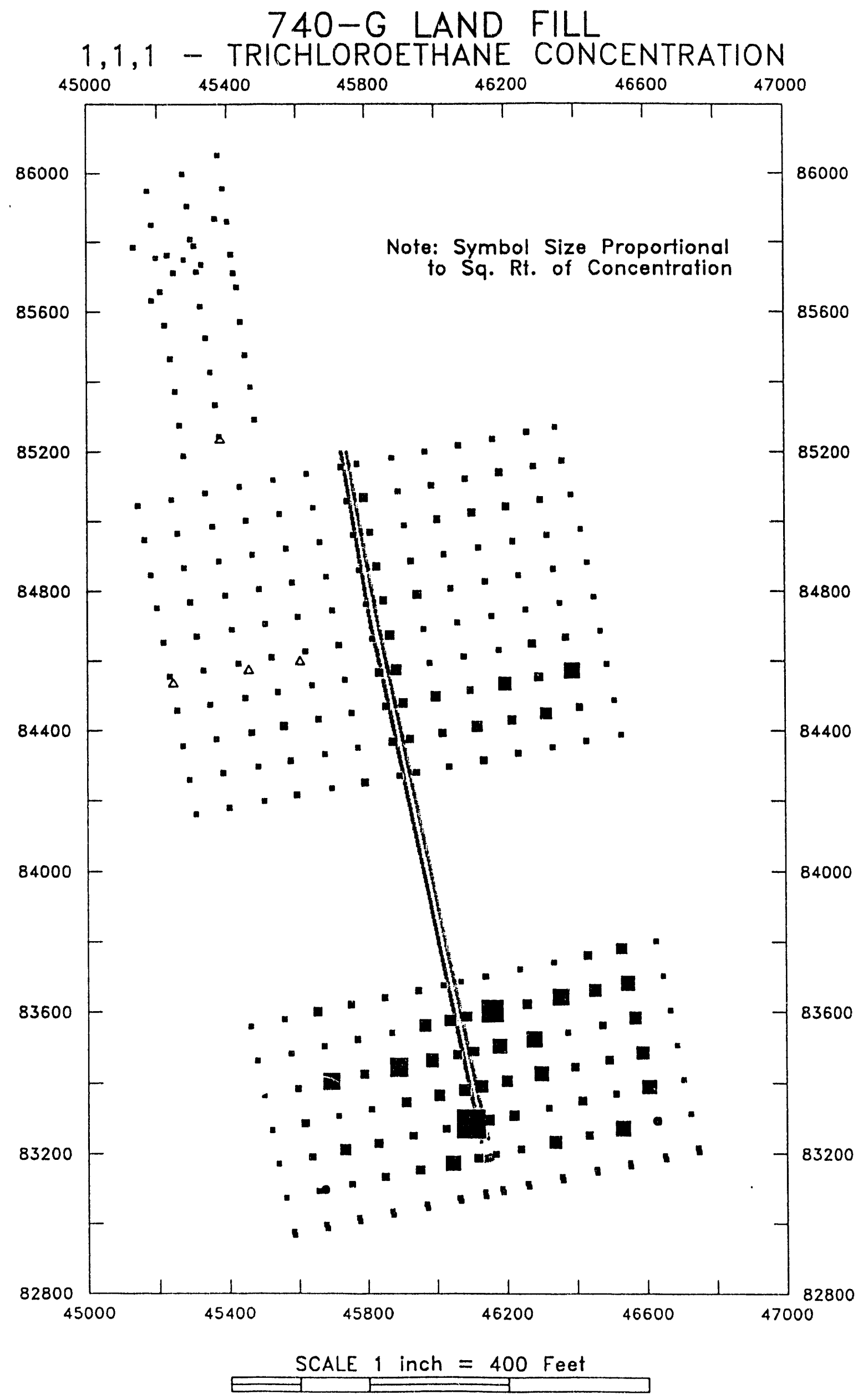

Figure 69. 1,1,1-Trichloroethane symbol Map 


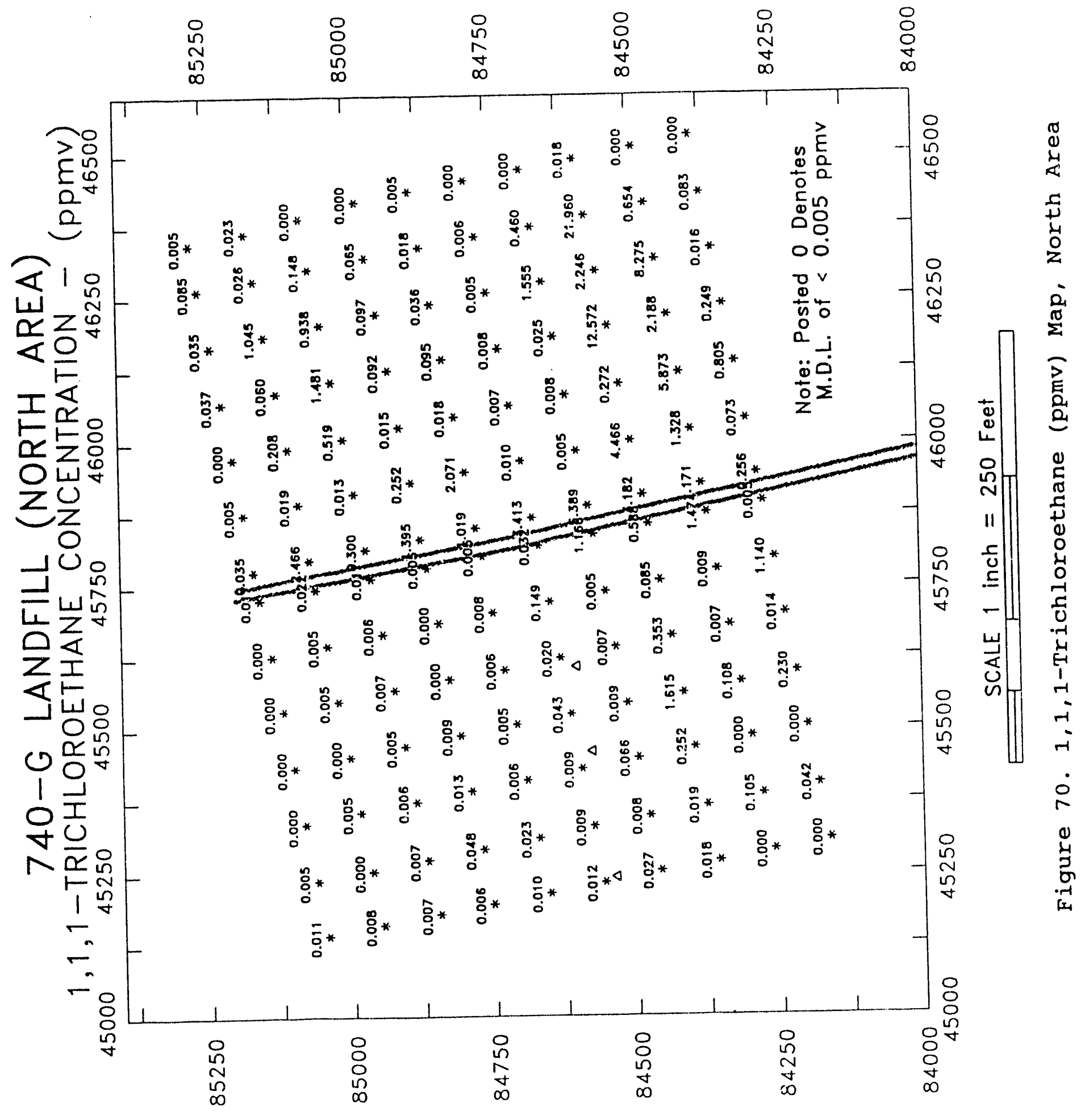




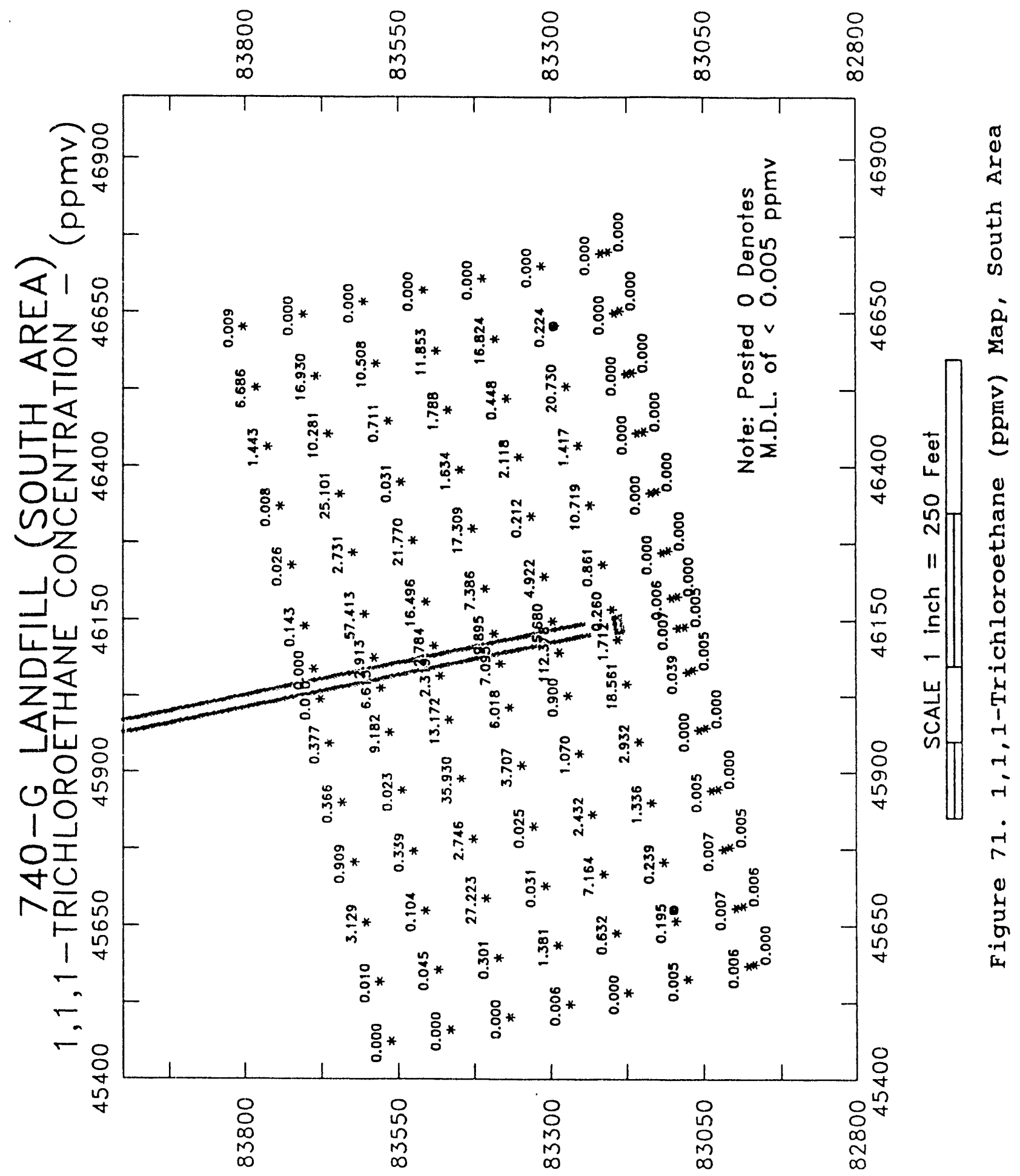




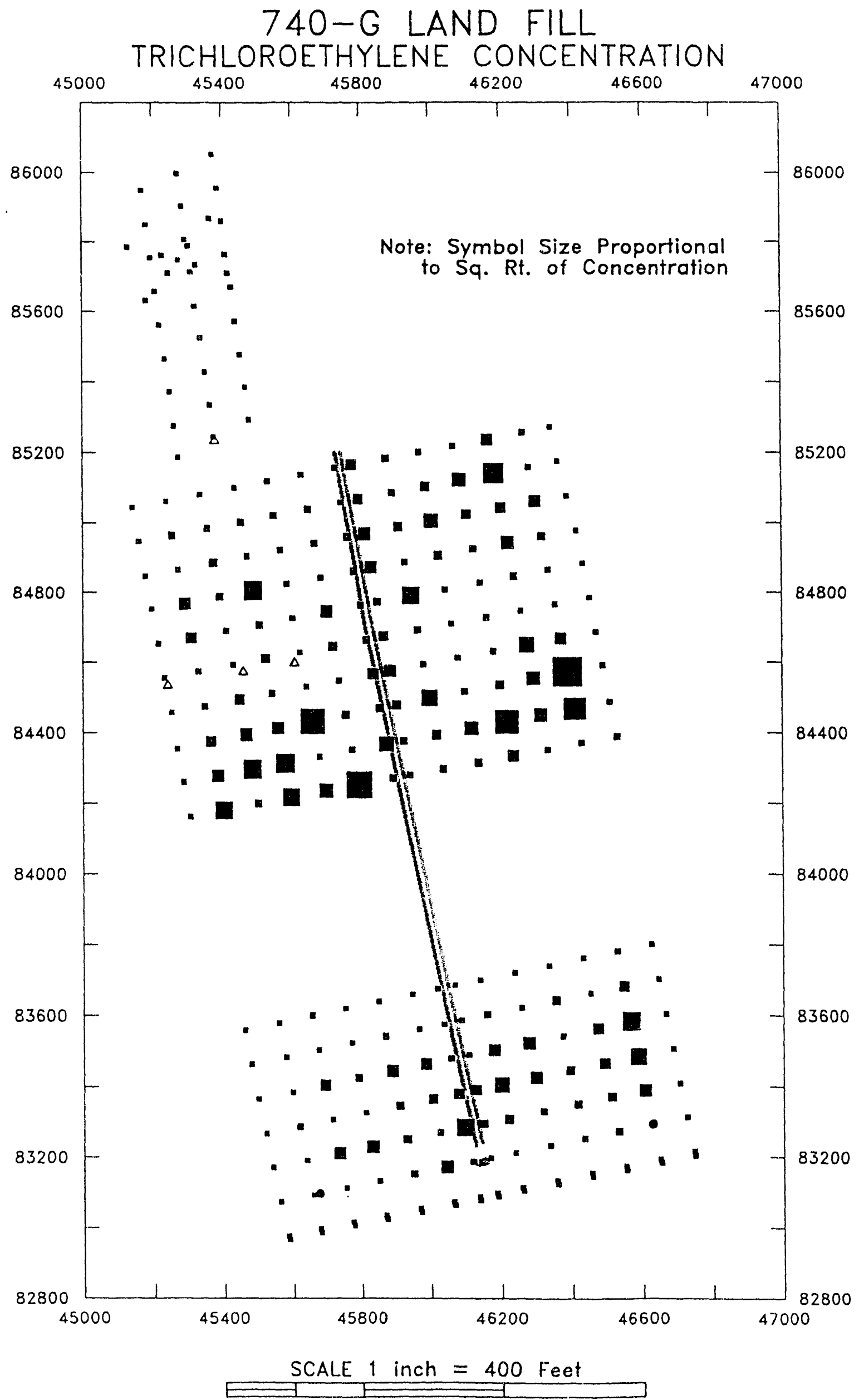

Figure 72. Trichloroethylene Symbol Map 


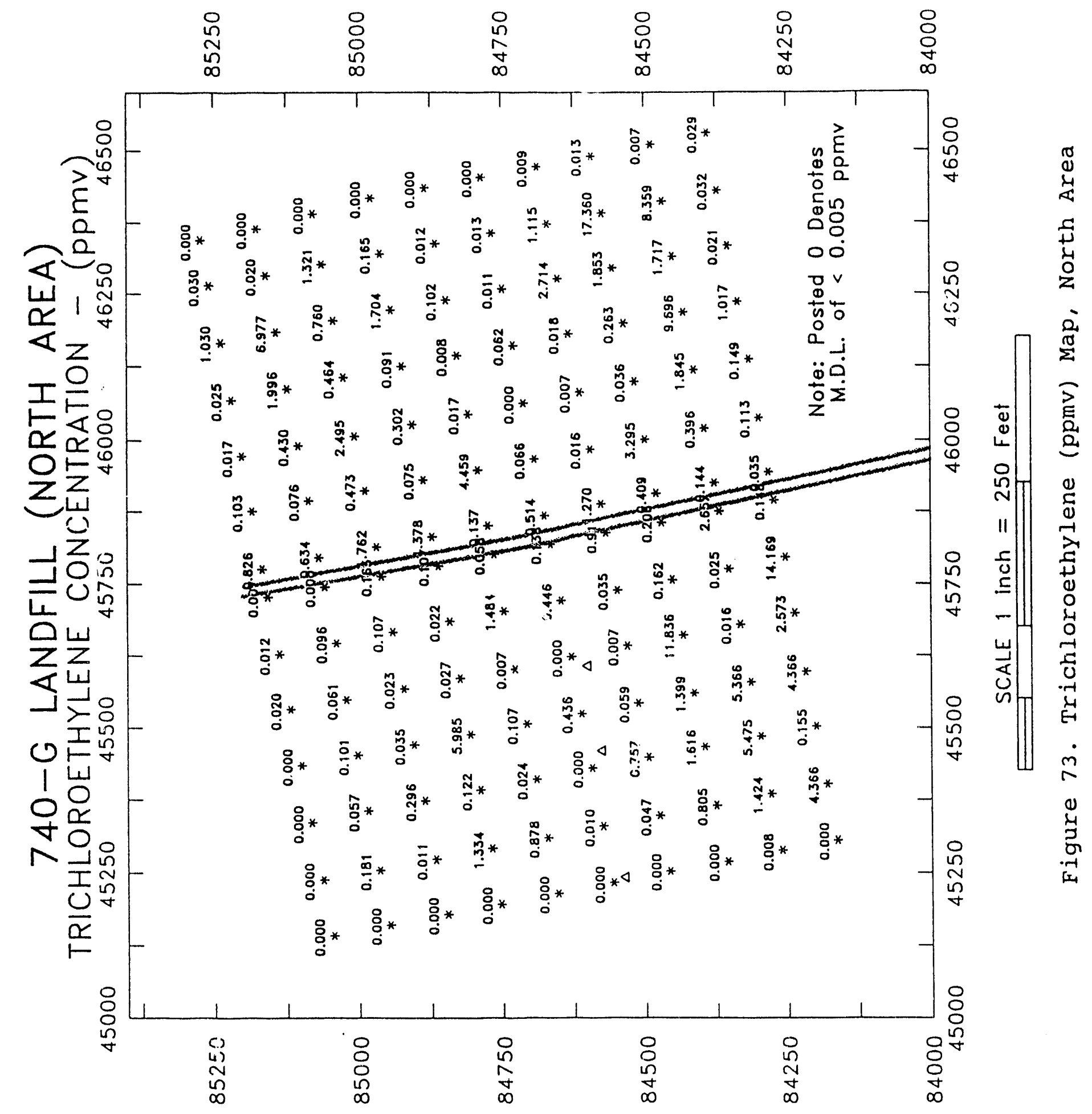




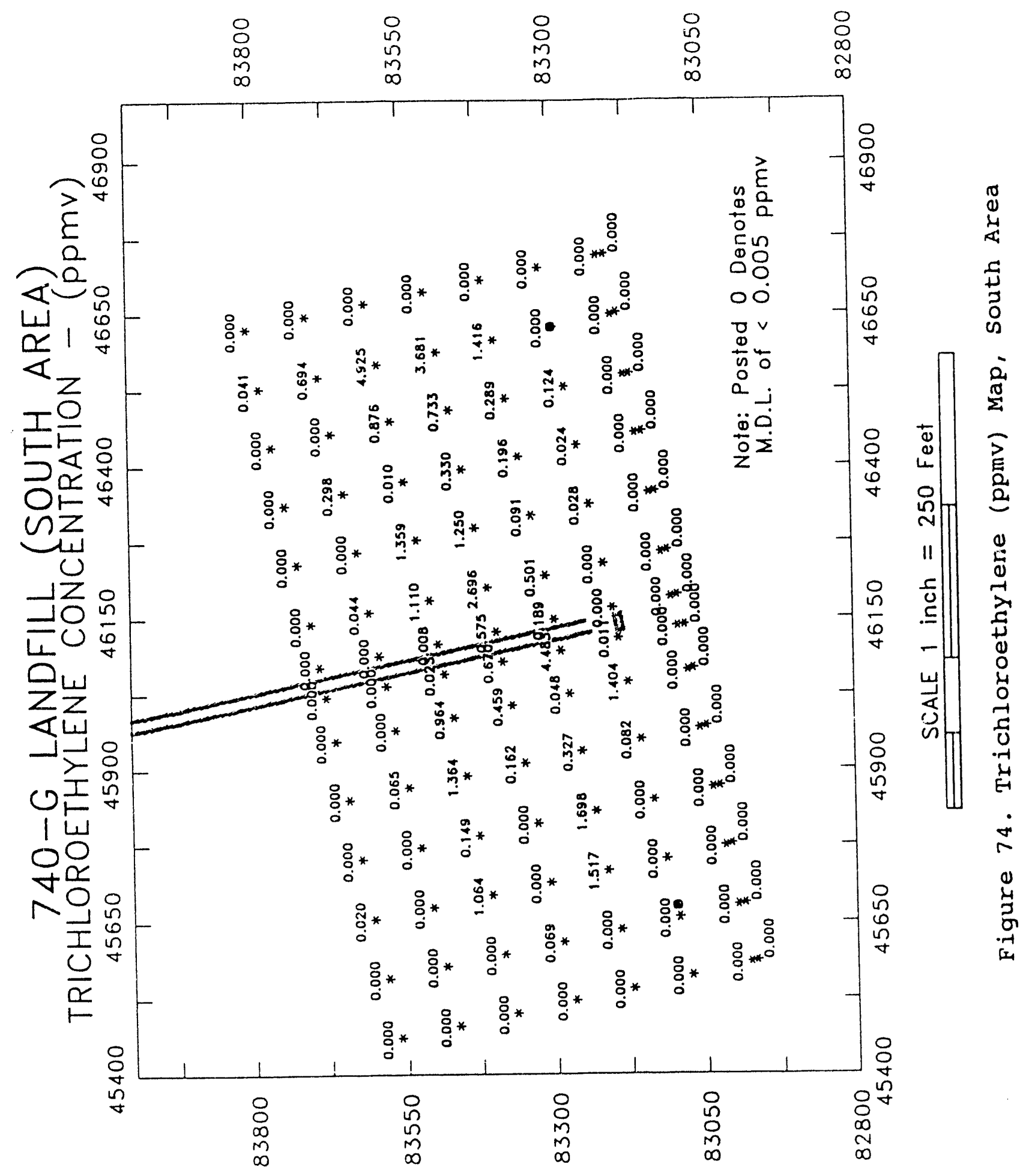




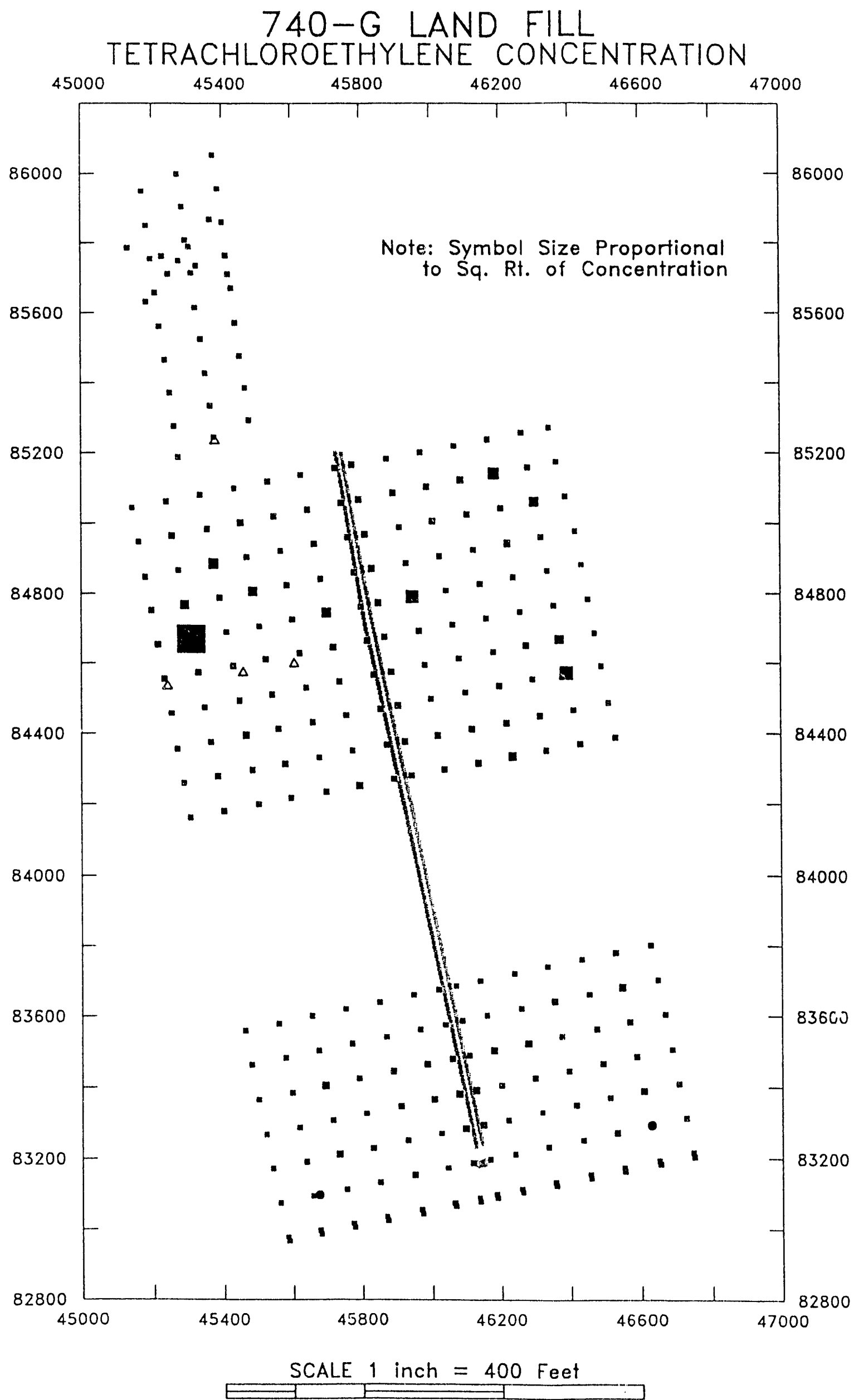

Figure 75. Tetrachloroethylene Symbol Map 


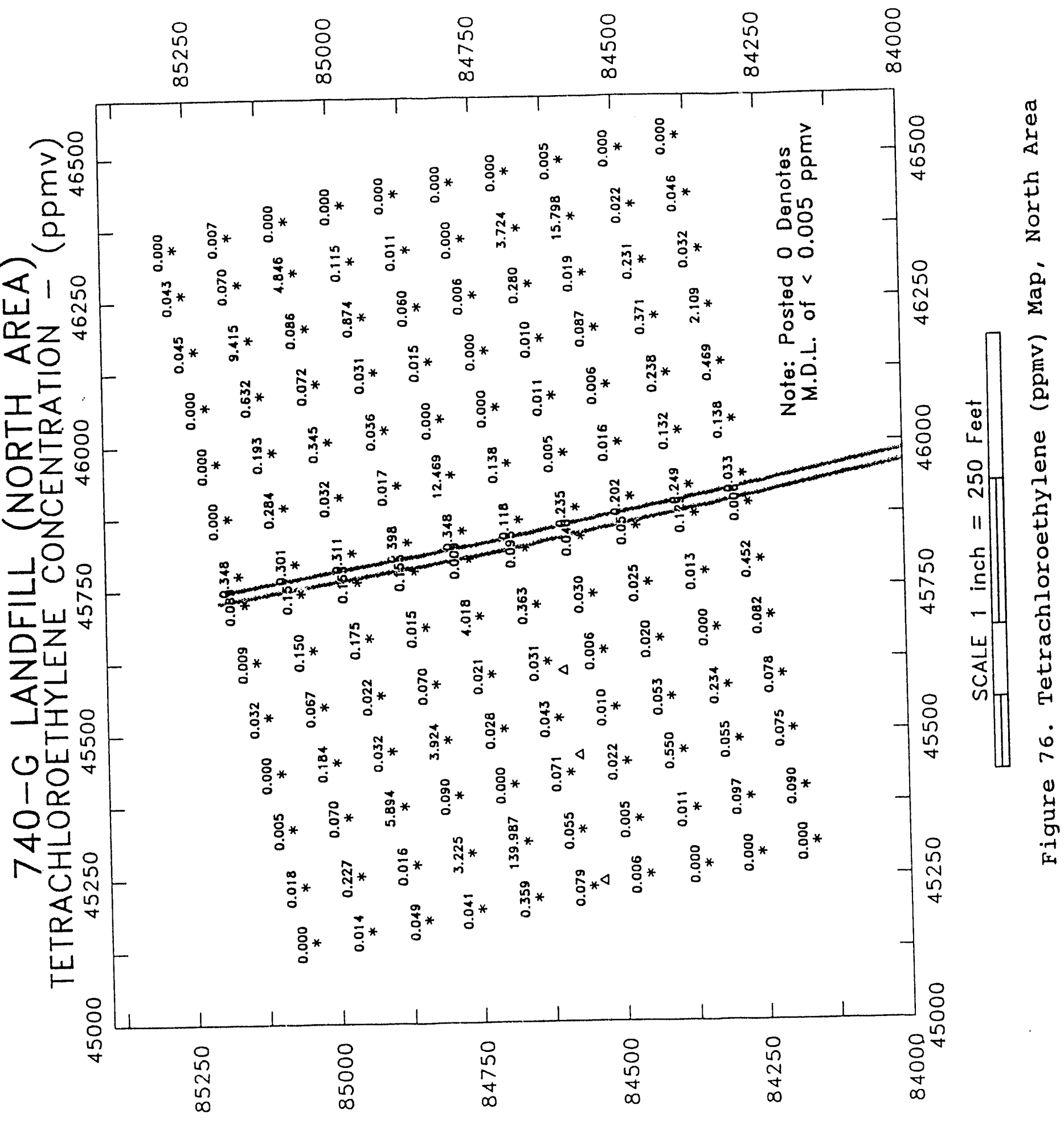




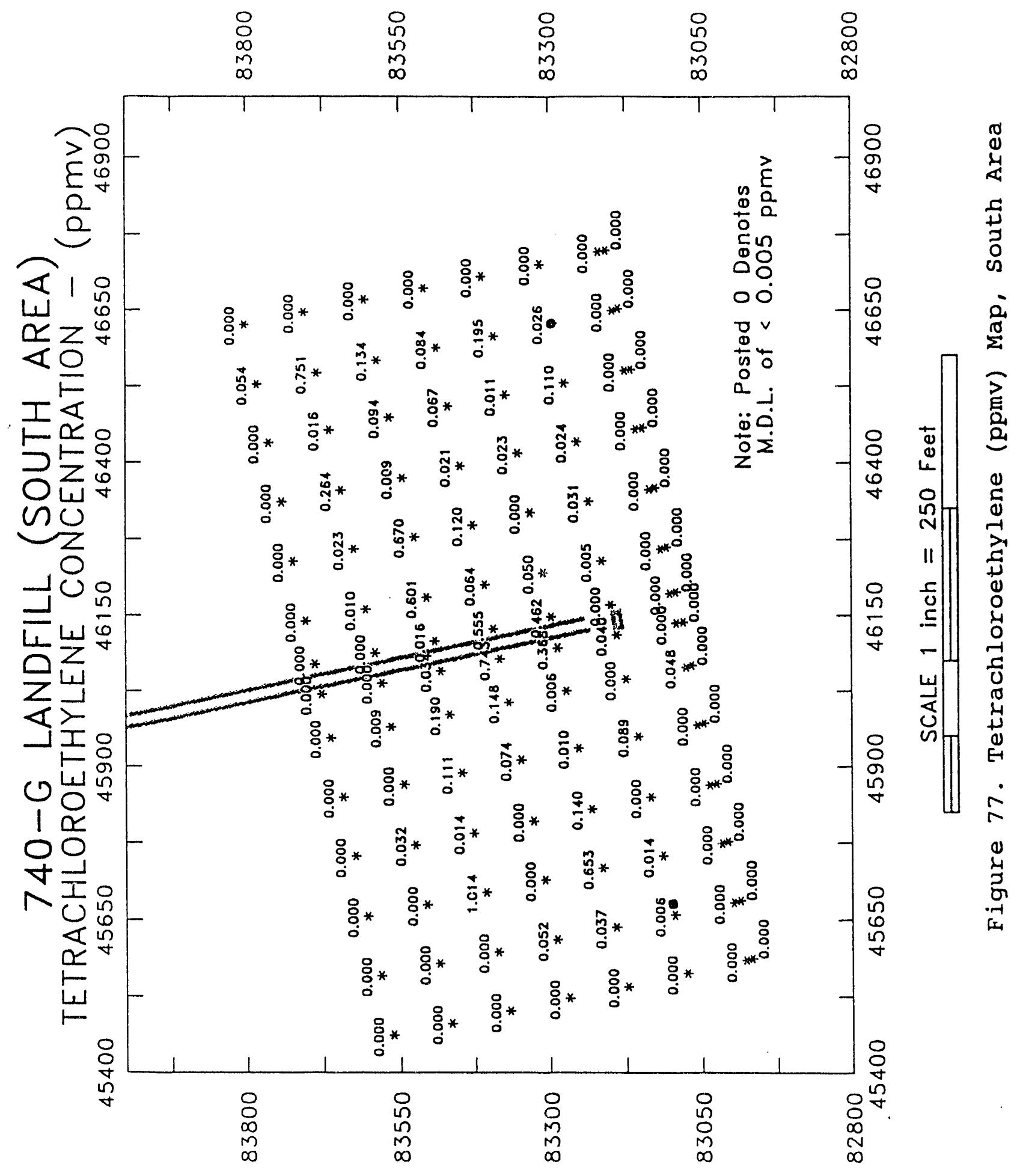




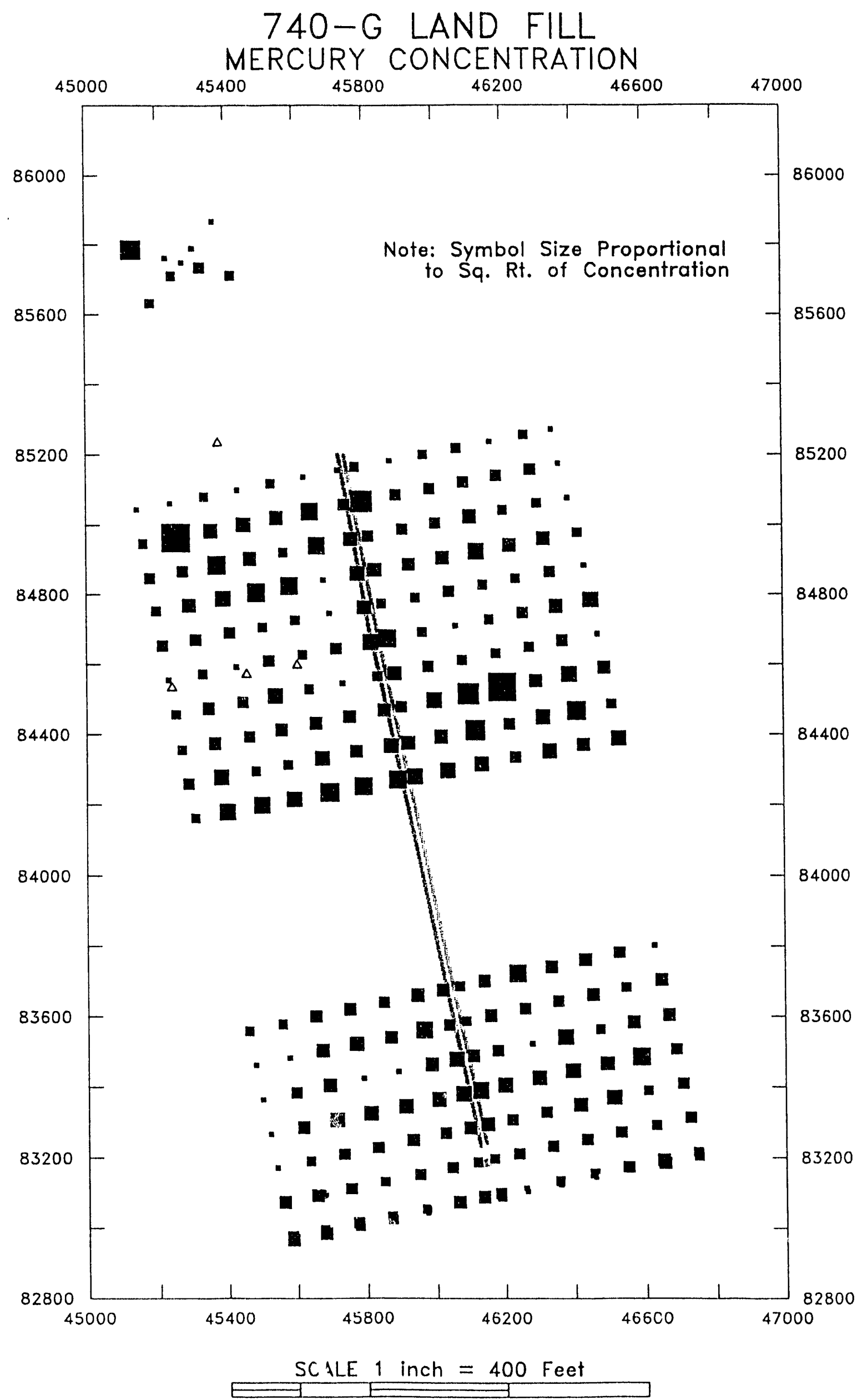

Figure 78. Mercury Symbol Map 


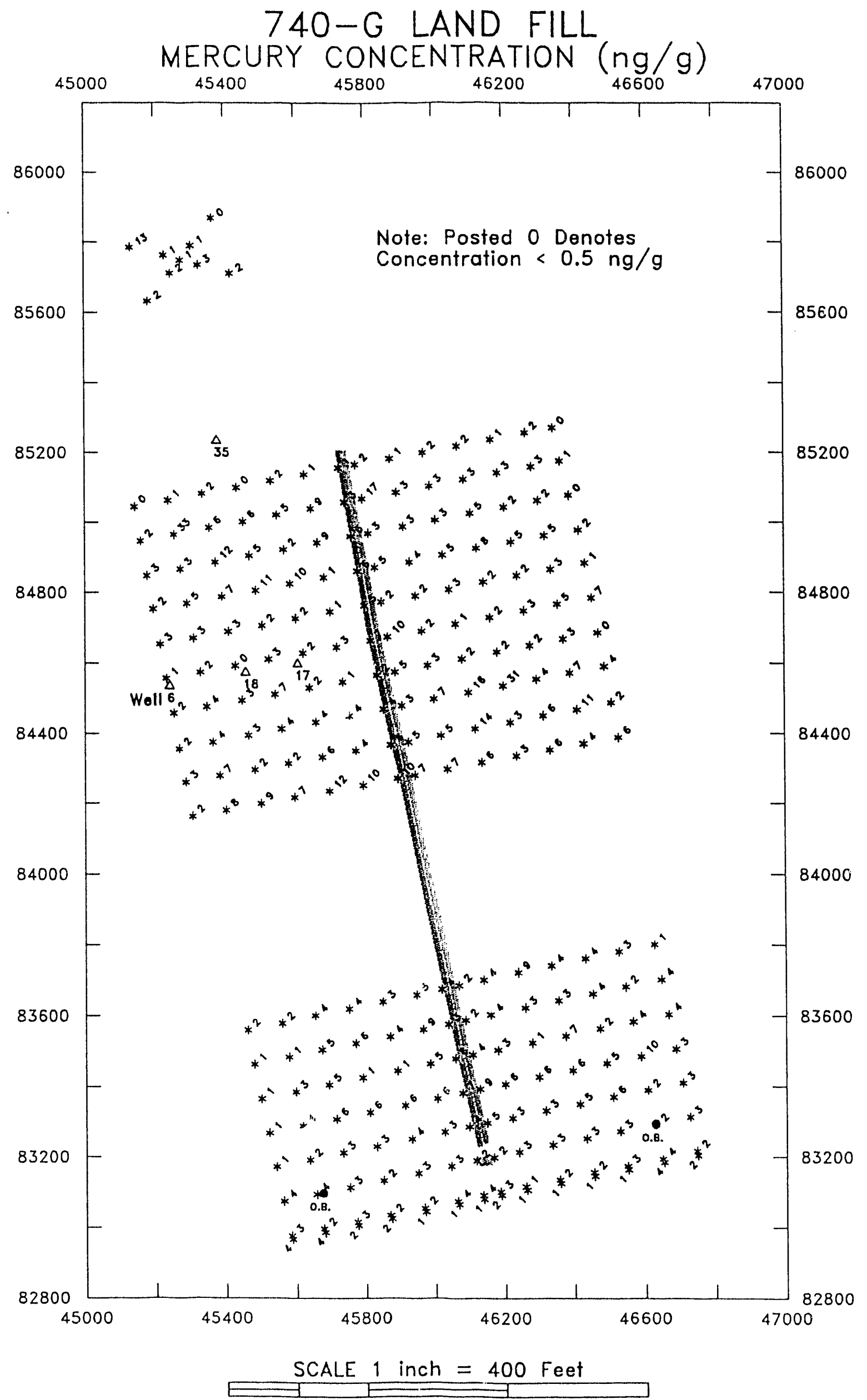

Figure 79. Mercury (ng/g) Map 
This page intentionally left blank. 
SAVANNAH RIVER SITE

740-G LANDFILL AREA .....

-... SOIL GAS CONCENTRATIONS (PPHY) .....

ETHYL M\&P- Osample \# pentane hexane heptane benzene octane toluene nowane benzene xylene Xylene decane

\begin{tabular}{|c|c|c|c|c|c|c|c|c|c|c|c|c|}
\hline $740-6$ & 1 & 5.29 & 0.16 & $<.07$ & $<.07$ & $<.07$ & $<.07$ & $<.07$ & $<.07$ & $<.07$ & $<.07$ & $<.07$ \\
\hline $740-6$ & 2 & 0.55 & 0.47 & 0.36 & $<.07$ & 0.84 & 0.52 & 1.13 & 0.34 & 0.96 & 0.40 & 0.48 \\
\hline $740-G$ & 3 & 0.37 & 0.30 & 0.22 & 0.82 & 0.29 & 0.31 & 1.05 & 0.17 & 0.90 & 0.48 & 0.64 \\
\hline $740-G$ & 4 & $<.07$ & 0.31 & 0.13 & $<.07$ & 0.52 & 0.26 & 1.07 & 0.67 & 1.07 & 0.33 & 0.82 \\
\hline $740-G$ & 5 & 0.20 & 0.73 & 0.31 & $<.07$ & 0.13 & 0.12 & 0.57 & 0.19 & 0.51 & 0.16 & 0.13 \\
\hline $740-G$ & 6 & 0.18 & 0.22 & 0.13 & $<.07$ & $<.07$ & 0.10 & $<.07$ & $<.07$ & $<.07$ & $<.07$ & $<.07$ \\
\hline $740-6$ & 7 & $<.07$ & $<.07$ & $<.07$ & $<.07$ & $<.07$ & $<.07$ & $<.07$ & $<.07$ & $<.07$ & $<.07$ & $<.07$ \\
\hline $740-G$ & 8 & $<.07$ & $<.07$ & $<.07$ & $<.07$ & $<.07$ & $<.07$ & $<.07$ & $<.07$ & $<.07$ & $<.07$ & $<.07$ \\
\hline $740-6$ & 9 & 1.17 & 1.85 & 1.52 & 0.14 & 1.65 & 0.67 & 2.61 & 0.20 & 1.14 & 0.52 & 0.47 \\
\hline $740-G$ & 10 & 0.47 & 1.26 & 1.53 & 0.30 & 4.42 & 1.72 & 9.72 & 0.67 & 3.10 & 1.51 & 2.93 \\
\hline $740-6$ & $10 A$ & 0.40 & 1.06 & $\therefore 42$ & 0.28 & 4.19 & 1.72 & 10.69 & 1.01 & 3.34 & 2.00 & 3.46 \\
\hline $740-G$ & 11 & 1.77 & 4.59 & 4.34 & 0.47 & 6.43 & 0.48 & $8=10$ & 0.34 & 2.89 & 2.88 & 3.50 \\
\hline $740-G$ & 12 & 1.64 & 4.18 & 4.10 & 0.29 & 2.83 & 0.89 & 3.39 & 0.27 & 3.34 & 0.66 & 1.39 \\
\hline $740-G$ & 13 & 1.81 & 3.42 & $3 . \pi 5$ & 0.38 & 5.48 & 2.21 & 2.49 & 0.18 & 1.17 & 0.52 & 0.41 \\
\hline $740-6$ & 14 & $<.07$ & $<.07$ & $<.07$ & $<.07$ & $<.07$ & $<.07$ & $<.07$ & $<.07$ & $<.07$ & $<.07$ & $<.07$ \\
\hline $740-G$ & 15 & 2.96 & 3.90 & 4.13 & 0.34 & 6.56 & 1.97 & 5.04 & 0.67 & 3.42 & 0.73 & 1.67 \\
\hline $740-\mathrm{G}$ & 16 & 0.69 & 2.32 & 3.61 & 0.38 & 4.29 & 22.45 & 4.89 & 4.89 & 12.54 & 4.00 & 2.21 \\
\hline $740-G$ & 17 & 0.29 & 1.15 & 1.73 & 0.17 & 1.59 & 3.52 & 3.74 & 4.24 & 9.04 & 2.94 & 1.93 \\
\hline $740-G$ & 18 & 0.99 & 1.88 & 1.83 & 0.36 & 1.35 & 4.05 & 2.09 & 1.86 & 3.60 & 1.09 & 0.70 \\
\hline $740-6$ & 19 & $<.07$ & 0.37 & 0.72 & 0.29 & 1.45 & 7.38 & 5.24 & 2.15 & 4.28 & 1.34 & 3.97 \\
\hline $740-G$ & 20 & $<.07$ & $<.07$ & $<.07$ & $<.07$ & $<.07$ & 0.08 & 0.11 & 0.08 & 0.15 & $<.07$ & 0.08 \\
\hline $740-6$ & $20 \mathrm{~A}$ & $<.07$ & 0.09 & $<.07$ & $<.07$ & $<.07$ & 0.16 & 0.09 & 0.22 & 0.28 & $<.07$ & $<.57$ \\
\hline $740-G$ & 21 & $<.07$ & $<.07$ & $<.07$ & $<.07$ & $<.07$ & $<.07$ & $<.07$ & $<.07$ & $<.07$ & $<.07$ & $<.07$ \\
\hline $740-6$ & 22 & $<.07$ & $<.07$ & $<.07$ & $<.07$ & $<.07$ & $<.07$ & $<.07$ & $<.07$ & $<.07$ & 0.15 & $<.07$ \\
\hline $740-\mathrm{G}$ & 23 & 2.94 & 6.36 & 5.00 & 0.74 & 6.78 & 16.54 & 6.68 & 11.12 & 26.81 & 6.12 & 2.28 \\
\hline $740-6$ & 24 & 0.38 & 2.17 & 1.46 & 0.24 & 2.77 & 2.23 & 4.43 & 1.90 & 5.12 & 1.75 & 5.97 \\
\hline $740-G$ & 25 & 0.97 & 1.97 & 1.37 & 0.56 & 1.31 & 15.69 & 0.64 & 1.40 & 1.37 & 0.40 & 0.52 \\
\hline $740-G$ & 26 & 0.16 & $<.07$ & $<.07$ & $<.07$ & $<.07$ & 0.10 & 0.11 & $<.07$ & 0.25 & 0.08 & 0.16 \\
\hline $740-G$ & 27 & 0.74 & 0.98 & 0.71 & 0.11 & 0.78 & 0.46 & 0.90 & 0.13 & 0.53 & 0.28 & 0.26 \\
\hline $740-G$ & 28 & 2.25 & 2.83 & 4.95 & 0.22 & 3.89 & 0.69 & 2.11 & 0.12 & 0.98 & 0.29 & 0.51 \\
\hline $740-G$ & 29 & 0.81 & 0.87 & 1.11 & 0.40 & 1.01 & 8.57 & 0.97 & 1.73 & 3.51 & 1.28 & 0.61 \\
\hline $740-G$ & 30 & 10.02 & 11.17 & 2.68 & 0.21 & 1.54 & 0.63 & 1.04 & 0.16 & 0.80 & 0.21 & 0.26 \\
\hline $740-0$ & $30 \mathrm{~A}$ & 21.76 & 25.18 & 6.10 & 0.54 & 3.68 & 4.97 & 2.71 & 1.08 & 2.58 & 0.65 & 0.47 \\
\hline $740-G$ & 31 & 0.68 & 0.18 & 0.09 & $<.07$ & $<.07$ & $<.07$ & $<.07$ & $<.07$ & $<.07$ & $<.07$ & $<.07$ \\
\hline $740-G$ & 32 & 2.07 & 2.18 & 1.33 & 0.23 & 1.30 & 0.58 & 1.87 & 0.19 & 1.43 & 0.55 & 0.42 \\
\hline $740-G$ & 33 & 0.36 & 0.07 & $<.07$ & $<.07$ & $<.07$ & 0.07 & $<.07$ & $<.07$ & $<.07$ & $<.07$ & $<.07$ \\
\hline $740-G$ & 34 & 4.18 & 0.21 & 0.29 & 0.16 & 0.85 & 11.32 & 2.55 & 1.94 & 4.45 & 1.58 & 1.09 \\
\hline $740-G$ & 35 & 0.08 & $<.07$ & $<.07$ & $<.07$ & $<.07$ & $<.07$ & $<.07$ & $<.07$ & 0.09 & $<.07$ & $<.07$ \\
\hline $740-G$ & 36 & $<.07$ & $<.07$ & $<.07$ & $<.07$ & $<.07$ & $<.07$ & $<.07$ & $<.07$ & $<.07$ & $<.07$ & $<.07$ \\
\hline $740-G$ & 37 & 0.44 & 0.38 & 0.18 & $<.07$ & 0.23 & $<.07$ & 1.29 & 0.47 & 2.62 & 0.68 & 0.33 \\
\hline $740-G$ & 38 & 0.08 & $<.07$ & $<.07$ & $<.07$ & $<.07$ & $<.07$ & $<.07$ & $<.07$ & $<.07$ & $<.07$ & $<.07$ \\
\hline $740-G$ & 39 & 1.19 & 2.50 & 0.48 & $<.07$ & 0.52 & 1.68 & 0.20 & 0.83 & 1.48 & 0.50 & $<.07$ \\
\hline $740-G$ & 40 & $<.07$ & 0.48 & $<.07$ & $<.07$ & 0.09 & $<.07$ & $<.07$ & $<.07$ & 0.08 & $<.07$ & $<.07$ \\
\hline $740-G$ & $40 \mathrm{~A}$ & $<.07$ & 0.69 & $<.07$ & $<.07$ & 0.08 & $<.07$ & $<.07$ & $<.07$ & 0.14 & $<.07$ & $<.07$ \\
\hline $740-G$ & 41 & 18.21 & 15.47 & 3.47 & 0.16 & 2.24 & 1.07 & 1.04 & 0.79 & 1.24 & 0.29 & 0.14 \\
\hline $740-G$ & 42 & 2.03 & 1.79 & 0.95 & $<.07$ & 0.68 & 0.44 & 0.93 & 0.45 & 1.14 & 0.23 & 0.26 \\
\hline $740-G$ & 43 & 5.60 & 3.18 & 1.59 & 0.44 & 1.52 & 1.90 & 1.83 & 0.93 & 3.87 & 1.85 & $0.5 \dot{5}$ \\
\hline $740-G$ & 44 & 0.37 & 0.33 & 0.33 & $<.07$ & 0.12 & 0.11 & 0.22 & 0.14 & 0.53 & $<.07$ & 0.25 \\
\hline $740-6$ & 4.5 & 0.89 & 31.08 & 1.34 & 0.26 & $<.07$ & 0.43 & $<.07$ & 0.67 & 1.43 & 0.38 & 0.11 \\
\hline $740-G$ & 46 & 0.37 & 0.92 & 0.13 & 0.12 & 0.17 & 3.02 & 0.90 & 0.99 & 0.62 & 0.31 & 0.31 \\
\hline $740-G$ & 47 & 2.51 & 6.07 & 14.45 & 0.66 & 12.75 & 6.59 & 5.28 & 11.53 & 3.50 & 0.89 & 0.23 \\
\hline $740-G$ & 48 & 1.58 & 0.78 & 0.51 & 0.21 & 0.14 & 0.56 & 0.37 & 1.20 & 0.32 & 0.13 & 0.12 \\
\hline
\end{tabular}

Table 1. Hydrocarbon Soil Gas Concentrations and system Blanks at the 740-G Sanitary Landfill 


\begin{tabular}{|c|c|c|c|c|c|c|c|c|c|c|}
\hline PENTAKE & HEXANE & HEPTANE & BENZENE & OCTANE & TOLUENE & NONANE & $\begin{array}{r}\text { ETHYL } \\
\text { BENZENE }\end{array}$ & $\begin{array}{r}\text { M\&P- } \\
\text { XYLENE }\end{array}$ & $\begin{array}{r}0- \\
\text { XYLENE }\end{array}$ & DECANE \\
\hline$<.07$ & $<.07$ & $<.07$ & $<.07$ & $<.07$ & $<.07$ & $<.07$ & $<.07$ & $<.07$ & $<.07$ & $<.07$ \\
\hline$<.07$ & $<.07$ & $<.07$ & $<.07$ & $<.07$ & $<.07$ & $<.07$ & $<.07$ & $<.07$ & $<.07$ & $<.07$ \\
\hline$<.07$ & $<.07$ & $<.07$ & $<.07$ & 0.08 & $<.07$ & $<.07$ & $<.07$ & $<.07$ & $<.07$ & $<.07$ \\
\hline 5.23 & 8.07 & 0.62 & 0.47 & 1.28 & 2.86 & 4.27 & 5.77 & 16.76 & 6.80 & 1.48 \\
\hline 14.63 & 6.02 & 1.90 & 0.14 & 0.69 & 2.48 & 0.78 & 1.50 & 3.70 & 0.45 & 0.30 \\
\hline 17.06 & 7.38 & 3.15 & 0.16 & 0.45 & 1.80 & 0.20 & 1.51 & 1.98 & 0.47 & 0.20 \\
\hline 51.66 & 9.55 & 1.87 & 1.16 & 1.08 & 5.18 & 0.65 & 1.36 & 0.99 & 0.32 & 0.10 \\
\hline 17.90 & 5.24 & 0.64 & 0.27 & 0.34 & 1.26 & 0.32 & 0.47 & 0.49 & 0.08 & 0.10 \\
\hline 0.91 & 0.15 & $<.07$ & $<.07$ & 0.21 & 0.23 & $<.07$ & $<.07$ & 0.08 & $<.07$ & $<.07$ \\
\hline 107.43 & 16.38 & 3.57 & 0.34 & 2.33 & 1.11 & 2.64 & 0.44 & 1.42 & 0.53 & 0.39 \\
\hline 0.58 & 0.13 & $<.07$ & 0.08 & $<.07$ & $<.07$ & 0.08 & $<.07$ & 0.10 & $<.07$ & 0.09 \\
\hline 0.19 & $<.07$ & $<.07$ & $<.07$ & $<.07$ & $<.07$ & $<.07$ & $<.07$ & $<.07$ & $<.07$ & $<.07$ \\
\hline 121.33 & 11.31 & 4.66 & 0.96 & 0.63 & 3.63 & 0.24 & 1.14 & 0.38 & 0.10 & $<.07$ \\
\hline 105.56 & 9.87 & 4.06 & 0.85 & 0.63 & 3.87 & 0.29 & 1.05 & 0.42 & 0.16 & $<.07$ \\
\hline 59.98 & 9.98 & 3.51 & 1.75 & 2.21 & 22.77 & 0.53 & 4.77 & 20.23 & 0.22 & $n .33$ \\
\hline 95.86 & 14.10 & 7.02 & 1.38 & 7.55 & 8.67 & 4.13 & 1.68 & 3.44 & 0.94 & 0.26 \\
\hline$<.07$ & $<.07$ & $<.07$ & $<.07$ & 0.11 & $<.07$ & $<.07$ & $<.07$ & 0.12 & $<.07$ & $<.07$ \\
\hline$<.07$ & $<.07$ & $<.07$ & $<.07$ & $<.07$ & $<.07$ & $<.07$ & $<.07$ & 0.07 & $<.07$ & $<.07$ \\
\hline 206.32 & 24.54 & 4.12 & 0.62 & 8.18 & 196.87 & 10.78 & 5.62 & 13.26 & 4.57 & 0.71 \\
\hline 46.44 & 28.16 & 3.34 & 2.30 & 4.06 & 120.88 & 13.63 & 7.30 & 20.57 & 5.69 & 2.39 \\
\hline 202.07 & 19.44 & 4.99 & 0.77 & 6.50 & 26.06 & 11.31 & 13.41 & 30.24 & 7.54 & 5.00 \\
\hline 355.61 & 96.98 & 63.50 & 8.63 & 23.40 & 77.46 & 15.53 & 7.18 & 16.64 & 6.17 & 3.57 \\
\hline 774.58 & 993.19 & 366.51 & 154.94 & 112.69 & 400.04 & 23.87 & 33.90 & 93.12 & 26.04 & 0.98 \\
\hline 69.94 & 15.38 & 9.96 & 1.66 & 20.65 & 9.80 & 12.30 & 2.89 & 6.97 & 2.44 & 2.05 \\
\hline 56.72 & 6.76 & 3.55 & 0.53 & 9.26 & 3.66 & 5.69 & 0.90 & 2.49 & 0.91 & 0.55 \\
\hline 0.37 & $<.07$ & $<.07$ & $<.07$ & $<.07$ & $<.07$ & $<.07$ & $<.07$ & 0.12 & $<.07$ & $<.07$ \\
\hline 4.64 & 4.15 & 1.71 & 0.53 & 0.48 & 0.25 & 0.29 & 0.21 & 0.44 & 0.10 & $<.07$ \\
\hline 18.98 & 7.40 & 0.79 & 0.15 & 0.4 .4 & 0.37 & 0.34 & 0.08 & 0.35 & 0.13 & $<.07$ \\
\hline 39.91 & 6.30 & 1.55 & 0.17 & 2.31 & 0.96 & 1.10 & 0.16 & 0.53 & 0.23 & 0.17 \\
\hline 32.43 & 20.88 & 0.89 & 0.10 & 0.91 & 0.97 & 0.24 & 3.95 & 0.24 & 0.08 & 0.36 \\
\hline 40.14 & 122.86 & 1.27 & 0.11 & 0.47 & 0.32 & 0.30 & 0.08 & 0.16 & 0.16 & 0.15 \\
\hline 0.09 & 0.09 & $<.07$ & $<.07$ & $<.07$ & $<.07$ & $<.07$ & $<.07$ & 0.12 & $<.07$ & $<.07$ \\
\hline$<.07$ & $<.07$ & $<.07$ & $<.07$ & $<.07$ & $<.07$ & $<.07$ & $<.07$ & $<.07$ & $<.07$ & $<.07$ \\
\hline 131.58 & 20.74 & 1.10 & 0.42 & 0.31 & 9.71 & 0.15 & 2.89 & 6.90 & 1.39 & $<.07$ \\
\hline 25.88 & 12.37 & 1.11 & 0.19 & 0.99 & 2.64 & 2.58 & 0.84 & 1.86 & 0.90 & 0.44 \\
\hline 19.47 & 6.78 & 0.84 & 0.17 & 0.47 & 0.66 & 2.73 & 0.48 & 1.26 & 0.77 & 0.33 \\
\hline 141.11 & 42.02 & 2.27 & 0.73 & 0.95 & 3.31 & 0.63 & 0.81 & 1.64 & 0.38 & 0.15 \\
\hline 38.41 & 6.32 & 1.47 & 0.14 & 1.32 & 1.00 & 1.95 & 0.40 & 1.49 & 0.69 & 0.48 \\
\hline 24.78 & 5.94 & 1.64 & 26.75 & 3.31 & 1.28 & 1.93 & 0.30 & 1.12 & 0.36 & 0.35 \\
\hline 0.39 & $<.07$ & $<.07$ & $<.07$ & $<.07$ & $<.07$ & $<.07$ & $<.07$ & 0.09 & $<.07$ & $<.07$ \\
\hline 1.24 & $<.07$ & $<.07$ & $<.07$ & $<.07$ & $<.07$ & $<.07$ & $<.07$ & $<.07$ & $<.07$ & $<.07$ \\
\hline 50.39 & 10.21 & 3.07 & 0.22 & 2.05 & 3.04 & 0.73 & 5.99 & 18.67 & 5.35 & $<.07$ \\
\hline 0.92 & $<.07$ & $<.07$ & $<.07$ & $<.07$ & $<.07$ & $<.07$ & $<.07$ & 0.08 & $<.07$ & $<.07$ \\
\hline 9.92 & $<.07$ & $<.07$ & $<.07$ & $<.07$ & $<.07$ & $<.07$ & $<.07$ & 0.09 & $<.07$ & $<.07$ \\
\hline 4.40 & $<.07$ & $<.07$ & $<.07$ & $<.07$ & $<.07$ & $<.07$ & $<.07$ & 0.10 & $<.07$ & $<.07$ \\
\hline 119.15 & 100.44 & 1.84 & 27.14 & 4.80 & 69.23 & 1.81 & 6.14 & 17.56 & 3.71 & 0.12 \\
\hline 154.98 & 124.70 & 2.51 & 21.02 & 7.31 & 68.42 & 3.01 & 8.01 & 23.42 & 5.37 & 0.19 \\
\hline$<.07$ & $<.07$ & $<.07$ & $<.07$ & $<.07$ & 0.09 & $<.07$ & $<.07$ & 0.15 & $<.07$ & $<.07$ \\
\hline$<.07$ & $<.07$ & $<.07$ & $<.07$ & 0.10 & $<.07$ & $<.07$ & $<.07$ & $<.07$ & $<.07$ & $<.07$ \\
\hline 14.18 & 13.98 & 0.90 & 0.08 & 0.32 & 0.22 & 0.44 & 0.17 & 0.51 & 0.15 & $<.07$ \\
\hline 2.70 & $<.07$ & $<.07$ & $<.07$ & $<.07$ & 0.08 & $<.07$ & $<.07$ & 0.07 & $<.07$ & $<.07$ \\
\hline 1.82 & 0.08 & $<.07$ & $<.07$ & $<.07$ & 0.39 & $<.07$ & 0.10 & 0.25 & $<.07$ & $<.07$ \\
\hline
\end{tabular}

1. Hydrocarbon Soil Gas Concentrations and System Blanks at the 740-G Sanitary Landfill 
-... SAVANMAH RIVER SITE ....

740-G LANDFILL AREA

.... SOIL GAS CONCENTRATIONS (PPHV)

\begin{tabular}{|c|c|c|c|c|c|c|c|c|c|c|c|c|}
\hline SAMPLE & 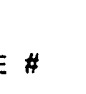 & PENTANE & HEXANE & HEPTANE & BENZENE & OCTANE & TOLUENE & NONANE & $\begin{array}{r}\text { ETHYL } \\
\text { BENZENE }\end{array}$ & $\begin{array}{r}\text { M\&P- } \\
\text { XYLENE }\end{array}$ & $\begin{array}{r}\text { O- } \\
\text { XYLENE }\end{array}$ & DECANE \\
\hline $740-G$ & 96 & 0.79 & $<.07$ & $<.07$ & $<.07$ & $<.07$ & $<.07$ & $<.07$ & $<.07$ & 0.09 & $<.07$ & $<.07$ \\
\hline $740-6$ & 97 & 123.45 & 3.70 & 1.51 & 3.08 & 0.44 & 8.56 & 1.10 & 3.77 & 3.04 & 1.00 & 0.70 \\
\hline $740-G$ & 98 & 3.54 & $<.07$ & $<.07$ & +07 & $<.07$ & $<.07$ & $<.07$ & $<.07$ & 0.09 & $<.07$ & $<.07$ \\
\hline $740-G$ & 99 & 1.80 & 0.07 & $<.07$ & $<.07$ & $<.07$ & $<.07$ & $<.07$ & $<.07$ & $<.07$ & $<.07$ & $<.07$ \\
\hline $740-G$ & 100 & 10.81 & 1.21 & 0.38 & 0.10 & 0.14 & 1.35 & $<.07$ & 0.15 & 0.36 & 0.09 & $<.07$ \\
\hline $740-\mathrm{G}$ & $100 \mathrm{~A}$ & 26.13 & 3.42 & 0.92 & 0.24 & 0.64 & 2.53 & 0.12 & 0.41 & 1.05 & 0.36 & $<.07$ \\
\hline $740-G .1$ & .101 & 0.09 & $<.07$ & $<.07$ & $<.07$ & $<.07$ & 0.21 & $<.07$ & $<.07$ & $<.07$ & $<.07$ & $<.07$ \\
\hline $740-6$ & 102 & 0.09 & $<.07$ & $<.07$ & $<.07$ & $<.07$ & $<.07$ & $<.07$ & $<.07$ & 0.08 & $<.07$ & $<.07$ \\
\hline $740-6$ & 103 & 0.28 & $<.07$ & $<.07$ & $<.07$ & $<.07$ & $<.07$ & $<.07$ & $<.07$ & 0.07 & $<.07$ & $<.07$ \\
\hline $740-G$ & 104 & 1.70 & 2.84 & 0.30 & $<.07$ & 0.12 & 0.08 & $<.07$ & $<.07$ & $<.07$ & $<.07$ & $<.07$ \\
\hline $740-6$ & 105 & $<.07$ & $<.07$ & $<.07$ & $<.07$ & 0.09 & $<.07$ & $<.07$ & $<.07$ & $<.07$ & $<.07$ & $<.07$ \\
\hline $740-G$ & 106 & $<.07$ & $<.07$ & $<.07$ & $<.07$ & $<.07$ & $<.07$ & $<.07$ & $<.07$ & $<.07$ & $<.07$ & $<.07$ \\
\hline $740-6$ & 107 & 12.81 & 2.49 & 0.21 & $<.07$ & 0.23 & 0.17 & 0.22 & $<.07$ & 0.16 & 0.08 & $<.07$ \\
\hline $740-G$ & 108 & 0.27 & $<.07$ & $<.07$ & $<.07$ & $<.07$ & $<.07$ & $<.07$ & $<.07$ & 0.08 & $<.07$ & $<.07$ \\
\hline $740-6$ & 109 & 4.21 & 1.03 & 0.57 & $<.07$ & 0.26 & 0.22 & 0.12 & $<.07$ & 0.10 & $<.07$ & $<.07$ \\
\hline $740-G$ & 110 & 1.25 & 0.31 & 0.13 & 0.12 & 0.52 & 2.25 & 0.11 & 1.03 & 0.28 & 0.07 & $<.07$ \\
\hline $740-6$ & $110 \mathrm{~A}$ & 0.72 & 0.13 & 0.11 & 0.11 & 0.22 & 7.06 & 0.22 & 0.19 & 0.22 & 0.11 & $<.07$ \\
\hline $740-6$ & 111 & 46.00 & 38.74 & 3.94 & 1.04 & 2.58 & 67.99 & 5.04 & 15.69 & 47.17 & 14.91 & 0.67 \\
\hline $740-6$ & 112 & 1.04 & $<.07$ & $<.07$ & $<.07$ & $<.07$ & 0.27 & $<.07$ & 0.13 & 0.48 & 0.16 & 0.07 \\
\hline $740-G$ & 113 & 23.63 & 5.54 & 0.87 & 0.08 & 1.13 & 0.79 & 0.95 & 0.34 & 1.42 & 0.42 & 0.23 \\
\hline $740-6$ & 114 & 0.31 & $<.07$ & $<.07$ & $<.07$ & $<.07$ & 0.35 & $<.07$ & $<.07$ & 0.08 & $<.07$ & $<.07$ \\
\hline $740-6$ & 115 & 188.06 & 37.25 & 2.54 & 0.61 & 0.95 & 6.08 & 0.27 & 0.75 & 1.58 & 0.34 & $<.07$ \\
\hline $740-6$ & 116 & 0.56 & $<.07$ & $<.07$ & $<.07$ & $<.07$ & $<.07$ & $<.07$ & $<.07$ & 0.09 & $<.07$ & $<.07$ \\
\hline $740-6$ & 117 & 115.26 & 16.64 & 4.59 & 2.14 & 1.94 & 6.88 & 1.22 & 5.20 & 9.30 & 1.13 & 0.12 \\
\hline $740-G$ & 118 & 22.79 & 4.21 & 1.34 & 0.08 & 1.84 & 1.43 & 1.65 & 0.20 & 0.85 & 0.58 & 0.37 \\
\hline $740-6$ & 119 & $<.07$ & $<.07$ & $<.07$ & $<.07$ & $<.07$ & $<.07$ & $<.07$ & $<.07$ & $<.07$ & $<.07$ & $<.07$ \\
\hline $740-G$ & 120 & $<.07$ & $<.07$ & $<.07$ & $<.07$ & $<.07$ & $<.07$ & $<.07$ & $<.07$ & $<.07$ & $<.07$ & $<.07$ \\
\hline $740-6$ & 121 & 2.20 & 0.65 & 0.49 & 0.13 & 0.39 & 0.67 & 0.57 & 0.20 & 0.40 & 0.20 & 0.10 \\
\hline $740-6$ & 122 & 1.87 & 3.09 & 0.39 & 0.14 & 0.27 & 1.90 & 0.14 & 0.41 & 0.46 & 0.14 & $<.07$ \\
\hline $740-G$ & 123 & 6.87 & 0.30 & 0.09 & $<.07$ & $<.07$ & 0.08 & $<.07$ & $<.07$ & $<.07$ & $<.07$ & $<.07$ \\
\hline $740-6$ & 124 & 164.65 & 22.58 & 3.69 & 0.13 & 1.54 & 0.48 & 2.59 & 0.27 & 0.70 & 0.43 & 0.16 \\
\hline $740-G$ & 125 & 175.14 & 42.56 & 5.78 & 0.27 & 6.74 & 2.07 & 2.64 & 0.68 & 1.25 & 0.73 & 0.33 \\
\hline $740-G$ & 126 & 32.04 & 14.29 & 2.47 & 0.28 & 2.64 & 0.85 & 2.17 & 0.22 & 0.91 & 0.52 & 0.25 \\
\hline $740-6$ & 127 & 12.92 & 4.97 & 1.18 & 0.22 & 1.71 & 6.91 & 0.95 & 0.82 & 1.50 & 0.52 & $<.07$ \\
\hline $740-6$ & 128 & $<.07$ & $<.07$ & $<.07$ & $<.07$ & $<.07$ & $<.07$ & $<.07$ & $<.07$ & $<.07$ & $<.07$ & $<.07$ \\
\hline $740-6$ & 129 & 49.61 & 38.79 & 2.82 & 0.08 & 8.43 & 2.58 & 1.44 & 0.13 & 0.30 & 0.19 & $<.07$ \\
\hline $740-G$ & $129 \mathrm{~A}$ & 60.17 & 59.28 & 3.30 & 0.10 & 11.22 & 4.43 & 2.15 & 0.50 & 0.62 & 0.31 & $<.07$ \\
\hline $740-G$ & 130 & 12.77 & 11.86 & 5.00 & 1.15 & 6.53 & 48.99 & 6.40 & 6.02 & 12.60 & 2.99 & 1.34 \\
\hline $740-G$ & 131 & 56.90 & 17.06 & 5.08 & 1.25 & 4.51 & 19.47 & 8.85 & 4.90 & 10.07 & 3.58 & 1.22 \\
\hline $740-G$ & $131 \mathrm{~A}$ & 25.69 & 7.53 & 2.42 & 0.86 & 2.16 & 12.15 & 3.99 & 2.71 & 5.25 & 2.01 & 0.85 \\
\hline $740-G$ & 132 & 0.27 & $<.07$ & $<.07$ & 0.14 & $<.07$ & $<.07$ & $<.07$ & $<.07$ & 0.12 & $<.07$ & 0.08 \\
\hline $740-G$ & 133 & $<.07$ & $<.07$ & $<.07$ & $<.07$ & $<.07$ & $<.07$ & $<.07$ & $<.07$ & 0.14 & $<.07$ & $<.07$ \\
\hline $740-G$ & 134 & $<.07$ & $<.07$ & $<.07$ & 0.07 & $<.07$ & $<.07$ & $<.07$ & $<.07$ & 0.07 & $<.07$ & $<.07$ \\
\hline $740-G$ & 135 & 6.63 & 0.10 & $<.07$ & 0.08 & 0.08 & 0.18 & 0.19 & $<.07$ & 0.12 & 0.12 & 0.09 \\
\hline $740-G$ & 136 & 58.87 & 14.76 & 7.36 & 0.60 & 4.06 & 1.43 & 1.39 & 3.07 & 1.55 & 1.25 & 0.09 \\
\hline $740-G$ & 137 & 6.66 & 0.10 & $<.07$ & 0.10 & 0.08 & $<.07$ & $<.07$ & 0.08 & 0.12 & $<.07$ & $<.07$ \\
\hline $740-G$ & 138 & 4.82 & 0.18 & 0.10 & 0.20 & 0.11 & 0.14 & $<.07$ & 0.14 & 0.18 & 0.23 & $<.07$ \\
\hline $740-G$ & 139 & 8.16 & 1.41 & 0.48 & 0.19 & 0.38 & 0.18 & 0.25 & $<.07$ & 0.15 & 0.08 & $<.07$ \\
\hline $740-G$ & 140 & 72.99 & 32.44 & 5.46 & 0.61 & 3.01 & 1.54 & 1.25 & 0.37 & 0.76 & 0.30 & 0.10 \\
\hline $740-6$ & $140 \mathrm{~A}$ & 64.21 & 28.52 & 4.77 & 0.54 & 2.61 & 2.01 & 1.15 & 0.52 & 0.97 & 0.34 & 0.12 \\
\hline $740-G$ & 141 & $<.07$ & $<.07$ & $<.07$ & $<.07$ & $<.07$ & $<.07$ & $<.07$ & $<.07$ & 0.07 & $<.07$ & $<.07$ \\
\hline $740-G$ & 142 & $<.07$ & $<.07$ & $<.07$ & $<.07$ & $<.07$ & $<.07$ & $<.07$ & $<.07$ & $<.07$ & $<.07$ & $<.07$ \\
\hline
\end{tabular}

Tabls 1. Hydrocarbon Soil Gas Concentrations and System Blanks at the 740-G Sanitary Landfill 
SAVANHAH RIVER SITE .....

740-G LANDFILL AREA .....

- SOIL GAS CONCENTRATIONS (PPHV)

\begin{tabular}{|c|c|c|c|c|c|c|c|c|c|c|c|c|}
\hline SAMPLE & $E \#$ & PENTANE & HEXANE & HEPTANE & BENZENE & OCTANE & TOLUENE & NONANE & $\begin{array}{r}\text { ETHYL } \\
\text { BENZENE }\end{array}$ & $\begin{array}{r}\text { M\&P- } \\
\text { XYLENE }\end{array}$ & $\begin{array}{r}0^{-} \\
\text {XYLENE }\end{array}$ & DECANE \\
\hline $740-G$ & 143 & $<.07$ & $<.07$ & $<.07$ & $<.07$ & $<.07$ & $<.07$ & $<.07$ & $<.07$ & $<.07$ & $<.07$ & $<.07$ \\
\hline $740-G$ & 144 & $<.07$ & $<.07$ & $<.07$ & $<.07$ & $<.07$ & $<.07$ & $<.07$ & $<.07$ & $<.07$ & $<.07$ & $<.07$ \\
\hline $740-G$ & 145 & $<.07$ & $<.07$ & $<.07$ & $<.07$ & $<.07$ & $<.07$ & $<.07$ & $<.07$ & $<.07$ & $<.07$ & $<.07$ \\
\hline $740-G$ & 146 & $<.07$ & $<.07$ & $<.07$ & $<.07$ & 0.09 & $<.07$ & $<.07$ & $<.07$ & 0.07 & $<.07$ & $<.07$ \\
\hline $740-G$ & 147 & $<.07$ & $<.07$ & $<.07$ & $<.07$ & 0.09 & $<.07$ & $<.07$ & $<.07$ & 0.07 & $<.07$ & $<.07$ \\
\hline $740-G$ & 148 & $<.07$ & $<.07$ & $<.07$ & $<.07$ & $<.07$ & $<.07$ & $<.07$ & $<.07$ & 0.07 & $<.07$ & $<.07$ \\
\hline $740-6$ & 149 & $<.07$ & $<.07$ & $<.07$ & $<.07$ & $<.07$ & $<.07$ & $<.07$ & $<.07$ & $<.07$ & $<.07$ & $<.07$ \\
\hline $740-G$ & 150 & $<.07$ & $<.07$ & $<.07$ & $<.07$ & 0.13 & $<.07$ & $<.07$ & $<.07$ & $<.07$ & $<.07$ & $<.07$ \\
\hline $740-G$ & 151 & $<.07$ & $<.07$ & $<.07$ & $<.07$ & $<.07$ & $<.07$ & $<.07$ & $<.07$ & $<.07$ & $<.07$ & $<.07$ \\
\hline $740-G$ & 152 & $<.07$ & $<.07$ & $<.07$ & $<.07$ & $<.07$ & $<.07$ & $<.07$ & $<.07$ & $<.07$ & $<.07$ & $<.07$ \\
\hline $740-G$ & 153 & $<.07$ & $<.07$ & $<.07$ & $<.07$ & $<.07$ & $<.07$ & $<.07$ & $<.07$ & $<.07$ & $<.07$ & $<.07$ \\
\hline $740-G$ & 154 & $<.07$ & $<.07$ & $<.07$ & $<.07$ & $<.07$ & $<.07$ & $<.07$ & $<.07$ & $<.07$ & $<.07$ & $<.07$ \\
\hline $740-6$ & 155 & $<.07$ & $<.07$ & $<.07$ & $<.07$ & $<.07$ & $<.07$ & $<.07$ & $<.07$ & $<.07$ & $<.07$ & $<.07$ \\
\hline $740-G$ & $155 \mathrm{~A}$ & $<.07$ & $<.07$ & $<.07$ & $<.07$ & $<.07$ & $<.07$ & $<.07$ & $<.07$ & $<.07$ & $<.07$ & $<.07$ \\
\hline $740-6$ & 156 & 0.21 & $<.07$ & $<.07$ & $<.07$ & 0.11 & $<.07$ & $<.07$ & $<.07$ & 0.12 & $<.07$ & $<.07$ \\
\hline $740-G$ & 157 & 1.97 & $<.07$ & $<.07$ & $<.07$ & $<.07$ & $<.07$ & $<.07$ & $<.07$ & 0.08 & $<.07$ & $<.07$ \\
\hline $740 \cdot G$ & 158 & 2.07 & $<.07$ & $<.07$ & $<.07$ & $<.07$ & $<.07$ & $<.07$ & $<.07$ & $<.07$ & $<.07$ & $<.07$ \\
\hline $7,40-6$ & 159 & 0.87 & $<.07$ & $<.07$ & $<.07$ & $<.07$ & $<.07$ & $<.07$ & $<.07$ & $<.07$ & $<.07$ & $<.07$ \\
\hline $740-6$ & 160 & 1.58 & $<.07$ & $<.07$ & $<.07$ & $<.07$ & $<.07$ & $<.07$ & $<.07$ & $<.07$ & $<.07$ & $<.07$ \\
\hline $740-G$ & $160 \mathrm{~A}$ & 1.80 & $<.07$ & $<.07$ & $<.07$ & $<.07$ & $<.07$ & $<.07$ & $<.07$ & $<.07$ & $<.07$ & $<.07$ \\
\hline $740-G$ & 161 & i.17 & $<.07$ & $<.07$ & $<.07$ & $<.07$ & $<.07$ & $<.07$ & $<.07$ & $<.07$ & $<.07$ & $<.07$ \\
\hline $740-G$ & 162 & 2.15 & $<.07$ & $<.07$ & $<.07$ & $<.07$ & $<.07$ & $<.07$ & $<.07$ & $<.07$ & $<.07$ & $<.07$ \\
\hline $740-6$ & 163 & 6.00 & $<.07$ & $<.07$ & $<.07$ & $<.07$ & $<.07$ & $<.07$ & $<.07$ & $<.07$ & $<.07$ & $<.07$ \\
\hline $740-G$ & 164 & 2.14 & $<.07$ & $<.07$ & $<.07$ & $<.07$ & $<.07$ & $<.07$ & $<.07$ & $<.07$ & $<.07$ & $<.07$ \\
\hline $740-G$ & 165 & 1.32 & $<.07$ & $<.07$ & $<.07$ & $<.07$ & $<.07$ & $<.07$ & $<.07$ & $<.07$ & $<.07$ & $<.07$ \\
\hline $740-6$ & 166 & 1.06 & $<.07$ & $<.07$ & $<.07$ & $<.07$ & $<.07$ & $<.07$ & $<.07$ & $<.07$ & $<.07$ & $<.07$ \\
\hline $740-G$ & 167 & 0.19 & $<.07$ & $<.07$ & $<.07$ & 0.08 & $<.07$ & $<.07$ & $<.07$ & $<.07$ & $<.07$ & $<.07$ \\
\hline $740-G$ & 168 & $<.07$ & $<.07$ & $<.07$ & $<.07$ & $<.07$ & $<.07$ & $<.07$ & $<.07$ & $<.07$ & $<.07$ & $<.07$ \\
\hline $740-6$ & 169 & $<.07$ & $<.07$ & $<.07$ & $<.07$ & $<.07$ & $<.07$ & $<.07$ & $<.07$ & $<.07$ & $<.07$ & $<.07$ \\
\hline $740-G$ & 170 & 0.25 & $<.07$ & $<.07$ & $<.07$ & $<.07$ & $<.07$ & $<.07$ & $<.07$ & $<.07$ & $<.07$ & $<.07$ \\
\hline $740-G$ & $170 \mathrm{~A}$ & 0.24 & $<.07$ & $<.07$ & $<.07$ & $<.07$ & $<.07$ & $<.07$ & $<.07$ & $<.07$ & $<.07$ & $<.07$ \\
\hline $740-6$ & 171 & 172.22 & 84.29 & 4.16 & 0.23 & 1.57 & 2.08 & 0.70 & 0.27 & 1.76 & 0.44 & 0.09 \\
\hline $740-G$ & 172 & 107.88 & 57.17 & 5.32 & 0.26 & 2.11 & 1.85 & 0.79 & 0.70 & 2.30 & 0.56 & $<.07$ \\
\hline $740-G$ & 173 & 6.94 & 0.16 & $<.07$ & $<.07$ & $<.07$ & $<.07$ & $<.07$ & $<.07$ & 0.13 & $<.07$ & $<.07$ \\
\hline $740-G$ & 174 & 4.01 & $<.07$ & $<.07$ & $<.07$ & $<.07$ & $<.07$ & $<.07$ & $<.07$ & 0.07 & $<.07$ & $<.07$ \\
\hline $740-6$ & 175 & 16.52 & 1.25 & 0.80 & 0.25 & 0.43 & 0.88 & 0.39 & 0.13 & 0.30 & 0.09 & $<.07$ \\
\hline $740-6$ & 176 & 3.84 & $<.07$ & $<.07$ & $<.07$ & $<.07$ & $<.07$ & $<.07$ & $<.07$ & 0.08 & $<.07$ & 0.12 \\
\hline $740-6$ & 177 & 9.98 & 1.09 & 0.13 & 0.10 & $<.07$ & 0.12 & $<.07$ & 0.07 & 0.58 & 0.13 & $<.07$ \\
\hline $740-G$ & 178 & 0.76 & $<.07$ & $<.07$ & $<.07$ & $<.07$ & $<.07$ & $<.07$ & $<.07$ & 0.07 & $<.07$ & $<.07$ \\
\hline $740-6$ & 179 & 9.68 & 2.12 & 0.07 & $<.07$ & $<.07$ & 0.21 & $<.07$ & $<.07$ & 0.10 & $<.07$ & 0.09 \\
\hline $740-G$ & 180 & 7.09 & 0.35 & 0.10 & 0.16 & $<.07$ & 0.10 & $<.07$ & $<.07$ & $<.07$ & $<.07$ & 0.11 \\
\hline $740-G$ & $180 \mathrm{~A}$ & 1.17 & 0.07 & $<.07$ & $<.07$ & $<.07$ & 0.14 & $<.07$ & $<.07$ & $<.07$ & $<.07$ & $<.07$ \\
\hline $740-G$ & 181 & 8.64 & $<.07$ & $<.07$ & $<.07$ & $<.07$ & $<.07$ & $<.07$ & $<.07$ & $<.07$ & $<.07$ & $<.07$ \\
\hline $740-G$ & 182 & $<.07$ & $<.07$ & $<.07$ & $<.07$ & $<.07$ & $<.07$ & $<.07$ & $<.07$ & $<.07$ & $<.07$ & $<.07$ \\
\hline $740-G$ & 183 & $<.07$ & $<.07$ & $<.07$ & $<.07$ & $<.07$ & $<.07$ & $<.07$ & $<.07$ & $<.07$ & $<.07$ & $<.07$ \\
\hline $740-G$ & 184 & 20.11 & $<.07$ & $<.07$ & $<.07$ & $<.07$ & $<.07$ & $<.07$ & $<.07$ & $<.07$ & $<.07$ & $<.07$ \\
\hline $740-G$ & 185 & 154.92 & 9.45 & 2.43 & 0.47 & 0.64 & 2.13 & 0.25 & 5.08 & 17.27 & 4.04 & $<.07$ \\
\hline $740-G$ & 186 & 201.48 & 26.02 & $<.07$ & $<.07$ & 1.21 & 0.34 & 0.45 & 0.77 & 0.75 & 0.29 & $<.07$ \\
\hline $740-6$ & 187 & 60.95 & 9.77 & 1.20 & 0.16 & 0.79 & 0.98 & 1.21 & 0.43 & 1.67 & 0.50 & 0.10 \\
\hline $740-G$ & 188 & 2.32 & $<.07$ & $<.07$ & 0.07 & $<.07$ & $<.07$ & $<.07$ & $<.07$ & 0.09 & $<.07$ & 0.07 \\
\hline $740-G$ & 189 & 3.51 & $<.07$ & $<.07$ & $<.07$ & $<.07$ & $<.07$ & $<.07$ & $<.07$ & 0.08 & $<.07$ & $<.07$ \\
\hline $740-G$ & 190 & 2.31 & $<.07$ & $<.07$ & $<.07$ & $<.07$ & $<.07$ & $<.07$ & $<.07$ & $<.07$ & $<.07$ & $<.07$ \\
\hline
\end{tabular}

Table 1. Hydrocarbon Soil Gas Concentrations and System Blanks at the 740-G Sanitary Landfill 
SAVANNAH RIVER SITE

.... 740-G LANDFILL AREA .....

..... SOIL GAS CONCENTRATIONS (PPMV) .....

\begin{tabular}{|c|c|c|c|c|c|c|c|c|c|c|c|}
\hline SAMPLE \# & PENTANE & HEXANE & HEPTANE & BENZENE & octAME & TOLUENE & NONANE & $\begin{array}{r}\text { ETHYL } \\
\text { DENZENE }\end{array}$ & $\begin{array}{r}\text { M\&P. } \\
\text { XYLENE }\end{array}$ & $\begin{array}{r}0- \\
\text { XYLENE }\end{array}$ & DECANE \\
\hline $740-G \quad 190 A$ & 2.26 & $<.07$ & $<.07$ & $<.07$ & $<.07$ & $<.07$ & $<.07$ & $<.07$ & $<.07$ & $<.07$ & $<.07$ \\
\hline 740-G 191 & 89.40 & 4.93 & $<.07$ & 0.18 & 0.60 & 1.15 & 0.99 & 2.74 & 7.83 & 2.63 & 0.11 \\
\hline $740-G \quad 192$ & 2.43 & $<.07$ & $<.07$ & $<.07$ & $<.07$ & $<.07$ & $<.07$ & $<.07$ & 0.24 & 0.07 & $<.07$ \\
\hline $740-G \quad 193$ & 0.14 & $<.07$ & $<.07$ & $<.07$ & $<.07$ & $<.07$ & $<.07$ & $<.07$ & 0.09 & $<.07$ & 0.12 \\
\hline $740-G \quad 194$ & 0.52 & $<.07$ & $<.07$ & $<.07$ & $<.07$ & $<.07$ & $<.07$ & $<.07$ & $<.07$ & $<.07$ & 8.07 \\
\hline $740-G \quad 195$ & 1.53 & $<., 77$ & $<.07$ & $<.07$ & $<.07$ & $<.07$ & $<.07$ & $<.07$ & 0.08 & $<.07$ & 0.10 \\
\hline $740-G \quad 196$ & $<.07$ & $<.07$ & $<.07$ & $<.07$ & $<.07$ & $<.07$ & $<.07$ & $<.07$ & $<.07$ & $<.07$ & 0.10 \\
\hline $740-6 \quad 197$ & $<.07$ & $<j 7$ & $<.07$ & $<.07$ & $<.07$ & $<.07$ & $<.07$ & $<.07$ & 0.10 & $<.07$ & $<.07$ \\
\hline $740-G \quad 198$ & 0.17 & $<.07$ & $<.07$ & $<.07$ & $<.07$ & $<.07$ & $<.07$ & $<.07$ & $<.07$ & $<.07$ & 0.11 \\
\hline $740-6 \quad 199$ & 153.82 & 11.83 & 3.45 & $<.07$ & 0.55 & 0.37 & 0.25 & $<.07$ & $<.07$ & $<.07$ & $<.07$ \\
\hline $740-6200$ & 84.88 & 16.28 & 1.21 & $<.07$ & 0.54 & 0.28 & 0.49 & 0.14 & 0.35 & $<.07$ & 0.15 \\
\hline $740-6200 \mathrm{~A}$ & 68.18 & 15.63 & 1.09 & 0.07 & 0.74 & 0.48 & 0.63 & 0.27 & 0.62 & 0.14 & 0.11 \\
\hline $740-G \quad 201$ & 149.48 & 13.39 & 3.79 & 0.11 & 1.49 & 1.04 & 1.25 & 1.05 & 2.31 & 0.48 & $<.07$ \\
\hline $740-G \quad 202$ & 14.85 & 3.35 & 0.47 & 0.29 & 0.13 & 0.12 & 0.24 & 0.12 & 0.13 & $<.07$ & $<.07$ \\
\hline $740-6 \quad 203$ & 0.55 & $<.07$ & $<.07$ & $<.07$ & $<.07$ & $<.07$ & $<.07$ & $<.07$ & $<.07$ & $<.07$ & $<.07$ \\
\hline $740-6204$ & 3.25 & $<.07$ & $<.07$ & $<.07$ & $<.07$ & $<.07$ & $<.07$ & $<.07$ & $<.07$ & $<.07$ & $<.07$ \\
\hline $7.40-G \quad 205$ & 77.68 & 8.09 & 1.49 & $<.07$ & 1.60 & 1.38 & 0.71 & 0.77 & 2.49 & 0.51 & $<.07$ \\
\hline $740-6206$ & 112.80 & 13.26 & 11.14 & 0.14 & 5.76 & 2.01 & 1.27 & 0.14 & 0.54 & 0.18 & $<.07$ \\
\hline $740-G \quad 207$ & 1.37 & 0.11 & 0.07 & $<.07$ & $<.07$ & $<.07$ & $<.07$ & $<.07$ & $<.07$ & $<.07$ & 0.10 \\
\hline $740-G \quad 208$ & 471.50 & 2.25 & 2.07 & 0.23 & 0.66 & 43.15 & 0.07 & 60.66 & 169.08 & 34.86 & $<.07$ \\
\hline $740-6 \quad 209$ & 9.60 & 0.15 & 0.20 & $<.07$ & 0.30 & 0.15 & $<.07$ & 0.30 & 1.10 & 0.31 & 0.08 \\
\hline $740-G \quad 210$ & $<.07$ & $<.07$ & $<.07$ & $<.07$ & $<.07$ & $<.07$ & $<.07$ & $<.07$ & $<.07$ & $<.07$ & 0.07 \\
\hline $740-G \quad 210 \mathrm{~A}$ & $<.07$ & $<.07$ & $<.07$ & $<.07$ & 0.21 & $<.07$ & $<.07$ & $<.07$ & $<.07$ & $<.07$ & $<.07$ \\
\hline $740-6211$ & $<.07$ & $<.07$ & $<.07$ & $<.07$ & $<.07$ & $<.07$ & $<.07$ & $<.07$ & $<.07$ & $<.07$ & $<.07$ \\
\hline $740-\dot{G} 212$ & 1.70 & $<.07$ & $<.07$ & $<.07$ & $<.07$ & $<.07$ & $<.07$ & $<.07$ & $<.07$ & $<.07$ & $<.07$ \\
\hline $740-6 \quad 213$ & 4.05 & 0.14 & $<.07$ & $<.07$ & $<.07$ & 0.07 & $<.07$ & $<.07$ & $<.07$ & $<.07$ & $<.07$ \\
\hline $740-6214$ & 134.67 & 1.24 & 0.65 & 0.25 & 0.33 & 1.08 & 0.18 & 0.75 & 2.95 & 0.52 & 0.07 \\
\hline $740-G \quad 215$ & 5.82 & 0.22 & $<.07$ & $<.07$ & $<.07$ & $<.07$ & 0.08 & $<.07$ & 0.11 & $<.07$ & 0.10 \\
\hline $740-G 216$ & 1.01 & $<.07$ & $<.07$ & $<.07$ & $<.07$ & $<.07$ & $<.07$ & $<.07$ & $<.07$ & $<.07$ & $<.07$ \\
\hline $740-0 \quad 217$ & 0.27 & $<.07$ & $<.07$ & $<.07$ & $<.07$ & $<.07$ & $<.07$ & $<.07$ & $<.07$ & $<.07$ & $<.07$ \\
\hline $740-G \quad 218$ & 0.15 & $<.07$ & $<.07$ & $<.07$ & $<.07$ & 0.15 & $<.07$ & $<.07$ & $<.07$ & $<.07$ & $<.07$ \\
\hline $740-G 219$ & 0.87 & $<.07$ & $<.07$ & $<.07$ & $<.07$ & $<.07$ & $<.07$ & $<.07$ & $<.07$ & $<.07$ & $<.07$ \\
\hline $740-G 220$ & 44.59 & 0.83 & 0.76 & 0.38 & 0.50 & 99.15 & 0.45 & 6.41 & 13.26 & 2.66 & 0.08 \\
\hline $740-G 220 \mathrm{~A}$ & 26.34 & 0.66 & 0.43 & 0.12 & 0.28 & 5.05 & 0.18 & 6.38 & 14.06 & 2.61 & $<.07$ \\
\hline $740-6221$ & 97.53 & 0.11 & $<.07$ & $<.07$ & 0.07 & 0.15 & $<.07$ & 0.17 & 0.48 & 0.11 & $<.07$ \\
\hline $740-G \quad 222$ & 0.87 & 0.07 & $<.07$ & $<.07$ & $<.07$ & $<.07$ & $<.07$ & $<.07$ & 0.19 & $<.07$ & $<.07$ \\
\hline $740-G 223$ & $<.07$ & $<.07$ & $<.07$ & $<.07$ & 0.09 & $<.07$ & $<.07$ & $<.07$ & 0.07 & $<.07$ & 0.08 \\
\hline $740-G 224$ & $<.07$ & $<.07$ & $<.07$ & $<.07$ & $<.07$ & $<.07$ & $<.07$ & $<.07$ & 0.08 & $<.07$ & $<.07$ \\
\hline $740-G 225$ & $<.07$ & $<.07$ & $<.07$ & $<.07$ & $<.07$ & $<.07$ & $<.07$ & $<.07$ & $<.07$ & $<.07$ & 0.07 \\
\hline $740-G 226$ & 0.09 & $<.07$ & $<.07$ & $<.07$ & $<.07$ & $<.07$ & $<.07$ & $<.07$ & 0.09 & $<.07$ & $<.07$ \\
\hline $740-6 \quad 227$ & 2.38 & $<.07$ & $<.07$ & $<.07$ & $<.07$ & $<.07$ & $<.07$ & $<.07$ & 0.08 & $<.07$ & 0.11 \\
\hline $740-6228$ & 0.76 & $<.07$ & $<.07$ & $<.07$ & $<.07$ & $<.07$ & $<.07$ & $<.07$ & 0.08 & $<.07$ & 0.10 \\
\hline $740-G \quad 229$ & 0.50 & $<.07$ & $<.07$ & $<.07$ & $<.07$ & $<.07$ & $<.07$ & $<.07$ & 0.11 & $<.07$ & 0.08 \\
\hline $740-6230$ & 0.07 & $<.07$ & $<.07$ & $<.07$ & $<.07$ & 0.08 & $<.07$ & $<.07$ & $<.07$ & $<.07$ & 0.09 \\
\hline $740-G 230 \mathrm{~A}$ & 0.09 & $<.07$ & $<.07$ & $<.07$ & $<.07$ & $<.07$ & $<.07$ & $<.07$ & $<.07$ & $<.07$ & 0.08 \\
\hline $740-G 231$ & $<.07$ & $<.07$ & $<.07$ & $<.07$ & $<.07$ & $<.07$ & $<.07$ & $<.07$ & 0.08 & $<.07$ & 0.07 \\
\hline $740-G 232$ & 0.66 & $<.07$ & $<.07$ & $<.07$ & $<.07$ & $<.07$ & $<.07$ & $<.07$ & $<.07$ & $<.07$ & 0.09 \\
\hline $740-6233$ & 1.15 & $<.07$ & $<.07$ & $<.07$ & $<.07$ & $<.07$ & $<.07$ & $<.07$ & $<.07$ & $<.07$ & $<.07$ \\
\hline $740-G 234$ & $<.07$ & $<.07$ & $<.07$ & $<.07$ & $<.07$ & $<.07$ & $<.07$ & $<.07$ & $<.07$ & $<.07$ & $<.07$ \\
\hline $740-G 235$ & 0.07 & $<.07$ & $<.07$ & $<.07$ & $<.07$ & $<.07$ & $<.07$ & $<.07$ & $<.07$ & $<.07$ & $<.07$ \\
\hline $740-G 236$ & 0.42 & $<.07$ & $<.07$ & $<.07$ & $<.07$ & $<.07$ & $<.07$ & $<.07$ & $<.07$ & $<.07$ & $<.07$ \\
\hline $740-G \quad 237$ & 1.00 & $<.07$ & $<.07$ & $<.07$ & $<.07$ & $<.07$ & $<.07$ & $<.07$ & 0.08 & $<.07$ & 0.09 \\
\hline
\end{tabular}

Table 1. Hydrocarbon Soil Gas Concentrations and System Blanks at the 740-G Sanitary Landfill 


\section{-... SAVANNAH RIVER SITE ..... \\ 740-G LANDFILL AREA -...}

-... SOIL GAS CONCENTRATIONS (PPHV)

ETHYL M\&P- $\quad 0$ sample \# pentane hexane heptane benzene octane toluene nonane benzene xylene xylene decane

\begin{tabular}{|c|c|c|c|c|c|c|c|c|c|c|c|}
\hline $740-G \quad 238$ & $<.07$ & $<.07$ & $<.07$ & $<.07$ & 0.17 & $<.07$ & $<.07$ & $<.07$ & $<.07$ & $<.07$ & $<.07$ \\
\hline $740-6239$ & $<.07$ & $<.07$ & $<.07$ & $<.07$ & $<.07$ & $<.07$ & $<.07$ & $<.07$ & $<.07$ & $<.07$ & $<.07$ \\
\hline $740-6240$ & $<.07$ & $<.07$ & $<.07$ & $<.07$ & $<.07$ & $<.07$ & $<.07$ & $<.07$ & $<.07$ & $<.07$ & $<.07$ \\
\hline $740-G 240 \mathrm{~A}$ & $<.07$ & $<.07$ & $<.07$ & $<.07$ & $<.07$ & $<.07$ & $<.07$ & $<.07$ & $<.07$ & $<.07$ & $<.07$ \\
\hline $740-G \quad 241$ & $<.07$ & $<.07$ & $<.07$ & $<.07$ & $<.07$ & $<.07$ & $<.07$ & $<.07$ & $<.07$ & $<.07$ & $<.07$ \\
\hline $740-G .242$ & $<.07$ & $<.07$ & $<.07$ & $<.07$ & $<.07$ & $<.07$ & $<.07$ & $<.07$ & $<.07$ & 0.07 & $<.07$ \\
\hline $740-G \quad 243$ & $<.07$ & $<.07$ & $<.07$ & $<.07$ & 0.09 & $<.07$ & $<.07$ & $<.07$ & $<.07$ & $<.07$ & $<.07$ \\
\hline $740-G 244$ & $<.07$ & 0.29 & $<.07$ & $<.07$ & $<.07$ & $<.07$ & $<.07$ & $<.07$ & $<.07$ & $<.07$ & $<.07$ \\
\hline $740-G \quad 245$ & $<.07$ & $<.07$ & $<.07$ & $<.07$ & $<.07$ & $<.07$ & $<.07$ & $<.07$ & 0.07 & $<.07$ & 0.08 \\
\hline $740-G 246$ & $<.07$ & $<.07$ & $<.07$ & $<.07$ & 0.12 & $<.07$ & $<.07$ & $<.07$ & 0.12 & $<.07$ & $<.07$ \\
\hline $740-G \quad 247$ & $<.07$ & $<.07$ & $<.07$ & $<.07$ & $<.07$ & $<.07$ & $<.07$ & $<.07$ & $<.07$ & $<.07$ & $<.07$ \\
\hline $740-G \quad 248$ & $<.07$ & $<.07$ & $<.07$ & $<.07$ & $<.07$ & $<.07$ & $<.07$ & $<.07$ & $<.07$ & $<.07$ & $<.07$ \\
\hline $74 \Gamma-G 249$ & $<.07$ & $<.07$ & $<.07$ & $<.07$ & 0.09 & $<.07$ & $<.07$ & $<.07$ & $<.07$ & $<.07$ & $<.07$ \\
\hline $740-6250$ & $<.07$ & $<.07$ & $<.07$ & $<.07$ & $<.07$ & $<.07$ & $<.07$ & $<.07$ & $<.07$ & 0.14 & $<.07$ \\
\hline $740-G \quad 250 \mathrm{~A}$ & $<.07$ & $<.07$ & $<.07$ & $<.07$ & $<.07$ & $<.07$ & $<.07$ & $<.07$ & $<.07$ & $<.07$ & $<.07$ \\
\hline $740-6251$ & $<.07$ & $<.07$ & $<.07$ & $<.07$ & $<.07$ & $<.07$ & $<.07$ & $<.07$ & $<.07$ & $<.07$ & $<.07$ \\
\hline $740-6 \quad 252$ & $<.07$ & $<.07$ & $<.07$ & $<.07$ & $<.07$ & $<.07$ & $<.07$ & $<.07$ & $<.07$ & $<.07$ & 0.07 \\
\hline $740-G 253$ & $<.07$ & $<.07$ & $<.07$ & $<.07$ & $<.07$ & $<.07$ & $<.07$ & $<.07$ & $<.07$ & $<.07$ & $<.07$ \\
\hline $740-6254$ & $<.07$ & $<.07$ & $<.07$ & $<.07$ & $<.07$ & $<.07$ & $<.07$ & $<.07$ & $<.07$ & $<.07$ & $<.07$ \\
\hline $740-G \quad 255$ & $<.07$ & $<.07$ & $<.07$ & $<.07$ & $<.07$ & $<.07$ & $<.07$ & $<.07$ & $<.07$ & $<.07$ & $<.07$ \\
\hline $740-6256$ & $<.07$ & $<.07$ & $<.07$ & $<.07$ & $<.07$ & $<.07$ & $<.07$ & $<.07$ & $<.07$ & $<.07$ & $<.07$ \\
\hline $740-6 \quad 257$ & $<.07$ & $<.07$ & $<.07$ & $<.07$ & $<.07$ & $<.07$ & $<.07$ & $<.07$ & $<.07$ & $<.07$ & $<.07$ \\
\hline $740-G \quad 258$ & $<.07$ & $<.07$ & $<.07$ & $<.07$ & $<.07$ & $<.07$ & $<.07$ & $<.07$ & $<.07$ & $<.07$ & $<.07$ \\
\hline $740-G \quad 259$ & $<.07$ & $<.07$ & $<.07$ & $<.07$ & $<.07$ & $<.07$ & $<.07$ & $<.07$ & $<.07$ & 0.10 & $<.07$ \\
\hline $740-G 260$ & $<.07$ & $<.07$ & $<.07$ & $<.07$ & $<.07$ & $<.07$ & $<.07$ & $<.07$ & $<.07$ & $<.07$ & 0.81 \\
\hline $740-G \quad 260 \mathrm{~A}$ & $<.07$ & $<.07$ & $<.07$ & $<.07$ & $<.07$ & $<.07$ & $<.07$ & $<.07$ & $<.07$ & $<.07$ & $<.07$ \\
\hline $740-G \quad 261$ & $<.07$ & $<.07$ & $<.07$ & $<.07$ & $<.07$ & $<.07$ & $<.07$ & $<.07$ & $<.07$ & $<.07$ & $<.07$ \\
\hline $740-G 262$ & $<.07$ & $<.07$ & $<.07$ & $<.07$ & $<.07$ & $<.07$ & $<.07$ & $<.07$ & $<.07$ & $<.07$ & $<.07$ \\
\hline $740-G \quad 263$ & $<.07$ & $<.07$ & $<.07$ & $<.07$ & $<.07$ & $<.07$ & $<.07$ & $<.07$ & $<.07$ & $<.07$ & $<.07$ \\
\hline $740-6264$ & $<.07$ & $<.07$ & $<.07$ & $<.07$ & $<.07$ & $<.07$ & $<.07$ & $<.07$ & $<.07$ & $<.07$ & $<.07$ \\
\hline $740-6265$ & $<.07$ & $<.07$ & $<.07$ & $<.07$ & $<.07$ & $<.07$ & $<.07$ & $<.07$ & $<.07$ & $<.07$ & $<.07$ \\
\hline $740-G 266$ & $<.07$ & $<.07$ & $<.07$ & $<.07$ & $<.07$ & $<.07$ & $<.07$ & $<.07$ & $<.07$ & $<.07$ & $<.07$ \\
\hline $740-6 \quad 267$ & $<.07$ & $<.07$ & $<.07$ & $<.07$ & $<.07$ & $<.07$ & $<.07$ & $<.07$ & $<.07$ & $<.07$ & $<.07$ \\
\hline $740-6 \quad 268$ & $<.07$ & $<.07$ & $<.07$ & $<.07$ & $<.07$ & $<.07$ & $<.07$ & $<.07$ & $<.07$ & $<.07$ & $<.07$ \\
\hline $740-G 269$ & $<.07$ & $<.07$ & $<.07$ & $<.07$ & $<.07$ & $<.07$ & $<.07$ & $<.07$ & $<.07$ & $<.07$ & $<.07$ \\
\hline $740-G 269 A$ & $<.07$ & $<.07$ & $<.07$ & $<.07$ & $<.07$ & $<.07$ & $<.07$ & $<.07$ & $<.07$ & $<.07$ & $<.07$ \\
\hline $740-G 270$ & $<.07$ & $<.07$ & $<.07$ & $<.07$ & $<.07$ & $<.07$ & $<.07$ & $<.07$ & $<.07$ & $<.07$ & $<.07$ \\
\hline $740-G \quad 271$ & $<.07$ & $<.07$ & $<.07$ & $<.07$ & $<.07$ & $<.07$ & $<.07$ & $<.07$ & $<.07$ & $<.07$ & $<.07$ \\
\hline $740-6272$ & $<.07$ & $<.07$ & $<.07$ & $<.0 i$ & $<.07$ & $<.07$ & $<.07$ & $<.07$ & $<.07$ & $<.07$ & $<.07$ \\
\hline $740-6273$ & $<.07$ & $<.07$ & $<.07$ & $<.07$ & $<.07$ & $<.07$ & $<.07$ & $<.07$ & $<.07$ & $<.07$ & $<.07$ \\
\hline $740-G 274$ & $<.07$ & $<.07$ & $<.07$ & $<.07$ & $<.07$ & $<.07$ & $<.07$ & $<.07$ & $<.07$ & $<.07$ & $<.07$ \\
\hline $740-6275$ & $<.07$ & $<.07$ & $<.07$ & $<.07$ & $<.07$ & $<.07$ & $<.07$ & $<.07$ & $<.07$ & $<.07$ & $<.07$ \\
\hline $740-G 276$ & $<.07$ & $<.07$ & $<.07$ & $<.07$ & $<.07$ & $<.07$ & $<.07$ & $<.07$ & $<.07$ & $<.07$ & $<.07$ \\
\hline $740-G \quad 277$ & $<.07$ & $<.07$ & $<.07$ & $<.07$ & $<.07$ & $<.07$ & $<.07$ & $<.07$ & $<.07$ & $<.07$ & $<.07$ \\
\hline $740-G 278$ & $<.07$ & $<.07$ & $<.07$ & $<.07$ & $<.07$ & $<.07$ & $<.07$ & $<.07$ & $<.07$ & $<.07$ & $<.07$ \\
\hline $740-G \quad 279$ & $<: 07$ & $<.07$ & $<.07$ & $<.07$ & $<.07$ & $<.07$ & $<.07$ & $<.07$ & $<.07$ & $<.07$ & $<.07$ \\
\hline $704-G 279 A$ & $<.07$ & $<.07$ & $<.07$ & $<.07$ & $<.07$ & $<.07$ & $<.07$ & $<.07$ & $<.07$ & $<.07$ & $<.07$ \\
\hline $740-G \quad 280$ & $<.07$ & $<.07$ & $<.07$ & $<.07$ & $<.07$ & $<.07$ & $<.07$ & $<.07$ & $<.07$ & $<.07$ & $<.07$ \\
\hline $740-G 281$ & $<.07$ & $<.07$ & $<.07$ & $<.07$ & $<.07$ & $<.07$ & $<.07$ & $<.07$ & $<.07$ & $<.07$ & $<.07$ \\
\hline $740-G \quad 282$ & $<.07$ & $<.07$ & $<.07$ & $<.07$ & $<.07$ & $<.07$ & $<.07$ & $<.07$ & $<.07$ & $<.07$ & $<.07$ \\
\hline $740-G \quad 283$ & $<.07$ & $<.07$ & $<.07$ & $<.07$ & $<.07$ & $<.07$ & $<.07$ & $<.07$ & $<.07$ & $<.07$ & $<.07$ \\
\hline $740-G 284$ & $<.07$ & $<.07$ & $<.07$ & $<.07$ & $<.07$ & $<.07$ & $<.07$ & $<.07$ & $<.07$ & $<.07$ & $<.07$ \\
\hline
\end{tabular}

Table 1. Hydrocarbon Soil Gas Concentrations and system Blanks at the 740-G Sanitary Landfill 


\begin{tabular}{|c|c|c|c|c|c|c|c|c|c|c|c|c|}
\hline SAMPLE & $\#$ & PENTANE & HEXANE & HEPTANE & BENZENE & OCTANE & TOLUENE & NONANE & $\begin{array}{r}\text { ETHYL } \\
\text { BENZENE }\end{array}$ & $\begin{array}{r}\text { M\&P- } \\
\text { XYLENE }\end{array}$ & $\begin{array}{r}0- \\
\text { XYLENE }\end{array}$ & DECANE \\
\hline $740-G$ & 285 & $<.07$ & $<.07$ & $<.07$ & $<.07$ & $<07$ & $<.07$ & $<.07$ & $<.07$ & $<.07$ & $<.07$ & $<.07$ \\
\hline $740-G$ & 286 & $<.07$ & $<.07$ & $<.07$ & $<.07$ & $<.07$ & $<.07$ & $<.07$ & $<.07$ & $<.07$ & $<.07$ & $<.07$ \\
\hline $740-6$ & 287 & $<.07$ & $<.07$ & $<.07$ & $<.07$ & $<.07$ & $<.07$ & $<.07$ & $<.07$ & $<.07$ & $<.07$ & $<.07$ \\
\hline $740-G$ & 288 & $<.07$ & $<.07$ & $<.07$ & $<.07$ & $<.07$ & $<.07$ & $<.07$ & $<.07$ & $<.07$ & $<.07$ & $<.07$ \\
\hline 740-G BLK & 1 & $<.07$ & $<.07$ & $<.07$ & $<.07$ & $<.07$ & $<.07$ & $<.07$ & $<.07$ & $<.07$ & $<.07$ & $<.07$ \\
\hline $740-G$ BLK & 2 & $<.07$ & $<.07$ & $<.07$ & $<.07$ & $<.07$ & $<.07$ & $<.07$ & $<.07$ & $<.07$ & $<.07$ & $<.07$ \\
\hline $740-6$ BLK & 3 & $<.07$ & $<.07$ & $<.07$ & $<.07$ & $<.07$ & $<.07$ & $<.07$ & $<.07$ & $<.07$ & $<.07$ & $<.07$ \\
\hline 740-G BLK & 4 & $<.07$ & $<.07$ & $<.07$ & $<.07$ & $<.07$ & $<.07$ & $<.07$ & $<.07$ & $<.07$ & $<.07$ & $<.07$ \\
\hline 740-G BLK & 5 & $<.07$ & $<.07$ & $<.07$ & $<.07$ & $<.07$ & $<.07$ & $<.07$ & $<.07$ & $<.07$ & $<.07$ & $<.07$ \\
\hline $740-G$ BLK & 6 & $<.07$ & $<.07$ & $<.07$ & $<.07$ & $<.07$ & $<.07$ & $<.07$ & $<.07$ & $<.07$ & $<.07$ & $<.07$ \\
\hline 740-G BLK & 7 & $<.07$ & $<.07$ & $<.07$ & $<.07$ & $<{ }_{4} 07$ & $<.07$ & $<.07$ & $<.07$ & 0.08 & $<.07$ & $<.07$ \\
\hline$\$ 40-G$ BLK & 8 & $<.07$ & 0.08 & $<.07$ & $<.07$ & 0.09 & 0.08 & $<.07$ & $<.07$ & 0.12 & $<.07$ & 0.08 \\
\hline $740-6$ BLK & 9 & $<.07$ & $<.07$ & $<.07$ & $<.07$ & $<.07$ & $<.07$ & $<.07$ & $<.07$ & 0.09 & $<.07$ & $<.07$ \\
\hline $740-G$ BLK & 10 & 0.10 & $<.07$ & $<.07$ & $<.07$ & $<.07$ & 0.07 & $<.07$ & $<.07$ & 0.13 & $<.07$ & $<.07$ \\
\hline $740-G$ BLK & 11 & 0.10 & 0.19 & $<.07$ & $<.0\rangle$ & $<.07$ & 0.31 & $<.07$ & 0.09 & 0.30 & $<.07$ & $<.07$ \\
\hline 740-G BLCK & 12 & $<.07$ & $<.07$ & $<.07$ & $<.07$ & $<.07$ & $<.07$ & $<.07$ & $<.07$ & 0.08 & $<.07$ & $<.07$ \\
\hline 740-G BLK & 13 & $<.07$ & $<.07$ & $<.07$ & $<.07$ & $<.07$ & $<.07$ & $<.07$ & $<.07$ & 0.08 & $<.07$ & $<.07$ \\
\hline $740-G^{\circ} B L K$ & 14 & $<.07$ & $<.07$ & $<.07$ & $<.07$ & $<.07$ & 0.08 & $<.07$ & $<.07$ & 0.13 & $<.07$ & $<.07$ \\
\hline $740-G$ BLK & 15 & $<.07$ & $<.07$ & $<.07$ & $<.07$ & $<.07$ & $<.07$ & $<.07$ & $<.07$ & 0.08 & $<.07$ & $<.07$ \\
\hline $740-G$ BLK & 16 & $<.07$ & $<.07$ & $<.07$ & $<.07$ & $<.07$ & $<.07$ & $<.07$ & $<.07$ & $<.07$ & $<.07$ & $<.07$ \\
\hline $740-G$ BLK & 17 & $<.07$ & $<.07$ & $<.07$ & $<.07$ & $<.07$ & $<.07$ & $<.07$ & $<.07$ & $<.07$ & $<.07$ & $<.07$ \\
\hline 740-G BLK & 18 & $<.07$ & $<.07$ & $<.07$ & $<.07$ & $<.07$ & $<.07$ & $<.07$ & $<.07$ & $<.07$ & $<.07$ & $<.07$ \\
\hline 740-G BLK & 19 & $<.07$ & $<.07$ & $<.07$ & $<.07$ & $<.07$ & $<.07$ & $<.07$ & $<.07$ & $<.07$ & $<.07$ & $<.07$ \\
\hline 740-G BLK & 20 & $<.07$ & $<.07$ & $<.07$ & $<.07$ & $<.07$ & $<.07$ & $<.07$ & $<.07$ & $<.07$ & $<.07$ & $<.07$ \\
\hline $740-G$ BLK & 21 & $<.07$ & $<.07$ & $<.07$ & $<.07$ & $<.07$ & $<.07$ & $<.07$ & $<.07$ & $<.07$ & $<.07$ & $<.07$ \\
\hline $740-G$ BLK & 22 & $<.07$ & $<.07$ & $<.07$ & $<.07$ & $<.07$ & $<.07$ & $<.07$ & $<.07$ & $<.07$ & $<.07$ & $<.07$ \\
\hline $740-G$ BLK & 23 & $<.07$ & $<.07$ & $<.07$ & $<.07$ & $<.07$ & $<.07$ & $<.07$ & $<.07$ & $<.07$ & $<.07$ & $<.07$ \\
\hline $740-G$ BLK & 24 & $<.07$ & $<.07$ & $<.07$ & $<.07$ & $<.07$ & $<.07$ & $<.07$ & $<.07$ & $<.07$ & $<.07$ & $<.07$ \\
\hline $740-G$ BLK & 25 & $<.07$ & $<.07$ & $<.07$ & $<.07$ & $<.07$ & $<.07$ & $<.07$ & $<.07$ & 0.17 & 0.10 & 0.08 \\
\hline $740-G$ BLK & 26 & $<.07$ & $<.07$ & $<.07$ & $<.07$ & $<.07$ & $<.07$ & $<.07$ & $<.07$ & $<.07$ & $<.07$ & $<.07$ \\
\hline $740-G$ BLK & 27 & $<.07$ & $<.07$ & $<.07$ & $<.07$ & $<.07$ & $<.07$ & $<.07$ & $<.07$ & $<.07$ & $<.07$ & $<.07$ \\
\hline $740-G$ BLK & 28 & $<.07$ & $<.07$ & $<.07$ & $<.07$ & $<.07$ & 0.10 & $<.07$ & $<.07$ & $<.07$ & $<.07$ & $<.07$ \\
\hline $740-G$ BLK & 29 & $<.07$ & $<.07$ & $<.07$ & $<.07$ & 0.23 & $<.07$ & $<.07$ & $<.07$ & $<.07$ & $<.07$ & $<.07$ \\
\hline 740-G BLK & 30 & $<.07$ & $<.07$ & $<.07$ & $<.07$ & $<.07$ & $<.07$ & $<.07$ & $<.07$ & $<.07$ & $<.07$ & $<.07$ \\
\hline $740-G$ BLK & 31 & $<.07$ & $<.07$ & $<.07$ & $<.07$ & $<.07$ & $<.07$ & $<.07$ & $<.07$ & $<.07$ & $<.07$ & $<.07$ \\
\hline $740-G$ BLK & 32 & $<.07$ & $<.07$ & $<.07$ & $<.07$ & $<.07$ & $<.07$ & $<.07$ & $<.07$ & $<.07$ & $<.07$ & $<.07$ \\
\hline $740-G$ BLK & 33 & $<.07$ & $<.07$ & $<.07$ & $<.07$ & $<.07$ & $<.07$ & $<.07$ & $<.07$ & $<.07$ & $<.07$ & $<.07$ \\
\hline $740-G$ BLK & 34 & $<.07$ & $<.07$ & $<.07$ & $<.07$ & $<.07$ & $<.07$ & $<.07$ & $<.07$ & $<.07$ & $<.07$ & $<.07$ \\
\hline
\end{tabular}


This page intentionally left blank. 


\begin{tabular}{|c|c|c|c|c|c|c|c|c|c|}
\hline & & & $\begin{array}{r}\ldots \\
\cdots \\
\cdots\end{array}$ & $\begin{array}{l}\text { SAVANNAH R } \\
740-G \text { LAND } \\
\text { GAS CONCE }\end{array}$ & $\begin{array}{l}\text { VER SITE } \\
\text { ILL AREA } \\
\text { TRATIONS }\end{array}$ & (PPMV) & -. & & \\
\hline SAMPLE & $E \# C$ & $\begin{array}{l}\text { VINYL } \\
\text { LORIDE }\end{array}$ & $\begin{array}{l}\text { METHYLENE } \\
\text { CHLORIDE }\end{array}$ & $\begin{array}{l}\text { TRANS } 1,2 \\
\text { DICHLORO } \\
\text { ETHYLENE }\end{array}$ & $\begin{array}{r}\text { CHLORO } \\
\text { FORM }\end{array}$ & $\begin{array}{r}111 \text { TRI } \\
\text { CHLORO } \\
\text { ETHANE }\end{array}$ & $\begin{array}{r}\text { CARBON } \\
\text { TETRA } \\
\text { CHLORIDE }\end{array}$ & $\begin{array}{l}\text { TRICLORO } \\
\text { ETHYLENE }\end{array}$ & $\begin{array}{r}\text { TETRA } \\
\text { CHLORO } \\
\text { ETHYLENE }\end{array}$ \\
\hline $740-G$ & 1 & $<1.0$ & $<1.0$ & $<.02$ & $<.005$ & 0.017 & $<.005$ & 0.007 & 0.089 \\
\hline $740-G$ & 2 & $<1.0$ & $<1.0$ & $<.02$ & $<.005$ & $<.005$ & $<.005$ & 0.012 & 0.009 \\
\hline $740-G$ & 3 & $<1.0$ & $<1.0$ & $<.02$ & $<.005$ & $<.005$ & $<.005$ & 0.020 & 0.032 \\
\hline $740-G$ & 4 & $<1.0$ & $<1.0$ & $<.02$ & $<.005$ & $<.005$ & $<.005$ & $<.005$ & 0.000 \\
\hline $740-6$ & 5 & $<1.0$ & $<1.0$ & $<.02$ & $<.005$ & $<.005$ & $<.005$ & $<.005$ & 0.005 \\
\hline $740-G$ & 6 & $<1.0$ & $<1.0$ & $<.02$ & $<.005$ & 0.005 & $<.005$ & $<.005$ & 0.018 \\
\hline $740-G$ & 7 & $<1.0$ & $<1.0$ & $<.02$ & $<.005$ & 0.011 & $<.005$ & $<.005$ & 0.000 \\
\hline $740-G$ & 8 & $<1.0$ & $<1.0$ & $<.02$ & $<.005$ & 0.008 & $<.005$ & $<.005$ & 0.014 \\
\hline $740-G$ & 9 & $<1.0$ & $<1.0$ & 0.10 & $<.005$ & $<.005$ & $<.005$ & 0.181 & 0.227 \\
\hline $740-G$ & 10 & $<1.0$ & $<1.0$ & 0.13 & $<.005$ & 0.005 & $<.005$ & 0.057 & 0.070 \\
\hline $740-G$ & $10 \mathrm{~A}$ & $<1.0$ & $<1.0$ & 0.12 & $<.005$ & 0.006 & $<.005$ & 0.053 & 0.071 \\
\hline $740-6$ & 11 & $<1.0$ & $<1.0$ & 0.26 & $<.005$ & $<.005$ & $<.005$ & 0.101 & 0.184 \\
\hline $740-G$ & 12 & $<1.0$ & $<1.0$ & 0.17 & $<.005$ & 0.005 & $<.005$ & 0.061 & 0.067 \\
\hline $740-G$ & 13 & $<1.0$ & $<1.0$ & 0.17 & $<.005$ & 0.005 & $<.005$ & 0.096 & 0.150 \\
\hline $740-G$ & 14 & $<1.0$ & $<1.0$ & $<.02$ & $<.005$ & 0.022 & $<.005$ & $<.005$ & 0.157 \\
\hline $740-G$ & 15 & $<1.0$ & $<1.0$ & 0.14 & $<.005$ & 0.017 & $<.005$ & 0.163 & 0.153 \\
\hline $740-G$ & 16 & $<1.0$ & 2.1 & 0.11 & $<.005$ & 0.006 & $<.005$ & 0.107 & 0.175 \\
\hline $740-6$ & 17 & $<1.0$ & $<1.0$ & 0.03 & $<.005$ & 0.0 .7 & $<.005$ & 0.023 & 0.022 \\
\hline $740-G$ & 18 & $<1.0$ & $<1.0$ & 0.15 & $<.005$ & 0.005 & $<.005$ & 0.035 & 0.032 \\
\hline $740-6$ & 19 & $<1.0$ & $<1.0$ & 0.07 & $<.005$ & 0.006 & $<.005$ & 0.296 & 5.894 \\
\hline $740-G$ & 20 & $<1.0$ & $<1.0$ & $<.02$ & $<.005$ & 0.007 & $<.005$ & 0.011 & 0.016 \\
\hline $740-6$ & $20 \mathrm{~A}$ & $<1.0$ & $<1.0$ & $<.02$ & $<.005$ & 0.008 & $<.005$ & 0.013 & 0.010 \\
\hline $740-G$ & 21 & $<1.0$ & $<1.0$ & $<.02$ & $<.005$ & 0.007 & $<.005$ & $<.005$ & 0.049 \\
\hline $740-G$ & 22 & $<1.0$ & $<1.0$ & $<.02$ & $<.005$ & 0.006 & $<.005$ & $<.005$ & 0.041 \\
\hline $740-6$ & 23 & $<1.0$ & 2.6 & 0.18 & $<.005$ & 0.048 & $<.005$ & 1.334 & 3.225 \\
\hline $740-G$ & 24 & $<1.0$ & 1.4 & 0.10 & $<.005$ & 0.013 & $<.005$ & 0.122 & 0.090 \\
\hline $740-6$ & 25 & $<1.0$ & 4.7 & 0.12 & $<.005$ & 0.009 & $<.005$ & 5.985 & 3.924 \\
\hline $740-6$ & 26 & $<1.0$ & $<1.0$ & $<.02$ & $<.005$ & $<.005$ & $<.005$ & 0.027 & 0.070 \\
\hline $740-G$ & 27 & $<1.0$ & $<1.0$ & 0.04 & $<.005$ & $<.005$ & $<.005$ & 0.022 & 0.015 \\
\hline $740-G$ & 28 & $<1.0$ & $<1.0$ & 0.08 & $<.005$ & 0.005 & $<.005$ & 0.107 & 0.155 \\
\hline $740-6$ & 29 & $<1.0$ & $<1.0$ & $<.02$ & $<.005$ & 0.005 & $<.005$ & 0.058 & 0.009 \\
\hline $740-6$ & 30 & $<1.0$ & $<1.0$ & $<.02$ & $<.005$ & 0.008 & $<.005$ & 1.484 & 4.018 \\
\hline $740-G$ & $30 \mathrm{~A}$ & $<1.0$ & 1.4 & 0.06 & $<.005$ & 0.039 & $<.005$ & 3.656 & 10.203 \\
\hline $740-G$ & 39 & $<1.0$ & $<1.0$ & $<.02$ & $<.005$ & 0.006 & $<.005$ & 0.007 & 0.021 \\
\hline $740-G$ & 32 & $<1.0$ & $<1.0$ & 0.03 & $<.005$ & 0.005 & $<.005$ & 0.107 & 0.028 \\
\hline $740-6$ & 33 & $<1.0$ & $<1.0$ & $<.02$ & $<.005$ & 0.006 & $<.005$ & 0.024 & 0.000 \\
\hline $740-G$ & 34 & $<1.0$ & 2.7 & $<.02$ & 0.295 & 0.023 & $<.005$ & 0.878 & 139.987 \\
\hline $740-C$ & 35 & $<1.0$ & 1.0 & $<.02$ & $<.005$ & 0.010 & $<.005$ & $<.005$ & 0.359 \\
\hline $740-G$ & 36 & $<1.0$ & 1.4 & $<.02$ & $<.005$ & 0.012 & $<.005$ & $<.005$ & 0.079 \\
\hline $740-G$ & 37 & $<1.0$ & $<1.0$ & $<.02$ & $<.005$ & 0.009 & $<.005$ & 0.010 & 0.055 \\
\hline $740-G$ & 38 & $<1.0$ & $<1.0$ & $<.02$ & $<.005$ & 0.009 & $<.005$ & $<.005$ & 0.071 \\
\hline $740-6$ & 39 & $<1.0$ & 1.1 & $<.02$ & $<.005$ & 0.043 & $<.005$ & 0.436 & 0.043 \\
\hline $740-G$ & 40 & $<1.0$ & 1.6 & $<.02$ & $<.005$ & 0.020 & $<.005$ & $<.005$ & 0.031 \\
\hline $740-G$ & $40 \mathrm{~A}$ & $<1.0$ & 1.3 & $<.02$ & $<.005$ & 0.015 & $<.005$ & $<.005$ & 0.014 \\
\hline $740 \cdot G$ & 41 & $<1.0$ & 1.9 & $<.02$ & $<.005$ & 0.149 & $<.005$ & 0.446 & 0.363 \\
\hline $740-G$ & 42 & $<1.0$ & 1.7 & $<.02$ & $<.005$ & 0.032 & $<.005$ & 0.138 & 0.095 \\
\hline $740-G$ & 43 & $<1.0$ & 2.6 & $<.02$ & $<.005$ & 1.168 & $<.005$ & 0.911 & 0.040 \\
\hline $740-G$ & 44 & $<1.0$ & $<1.0$ & $<.02$ & $<.005$ & 0.005 & $<.005$ & 0.035 & 0.030 \\
\hline $740-G$ & 45 & $<1.0$ & $<1.0$ & $<.02$ & $<.005$ & 0.007 & $<.005$ & 0.007 & 0.006 \\
\hline $740-G$ & 46 & $<1.0$ & $<1.0$ & $<.02$ & $<.005$ & 0.009 & $<.005$ & 0.059 & 0.010 \\
\hline $740 . G$ & 47 & $<1.0$ & $<1.0$ & 0.02 & $<.005$ & 0.066 & $<.005$ & 0.757 & 0.022 \\
\hline $740-G$ & 48 & $<1.0$ & $<1.0$ & $<.02$ & $<.005$ & 0.008 & $<.005$ & 0.047 & 0.005 \\
\hline
\end{tabular}

* SeE Section III. o.

Table 2. Chlorinated Hydrocarbon Soil Gas Concentrations and System Blanks at the 740-G Sanitary Landfill 


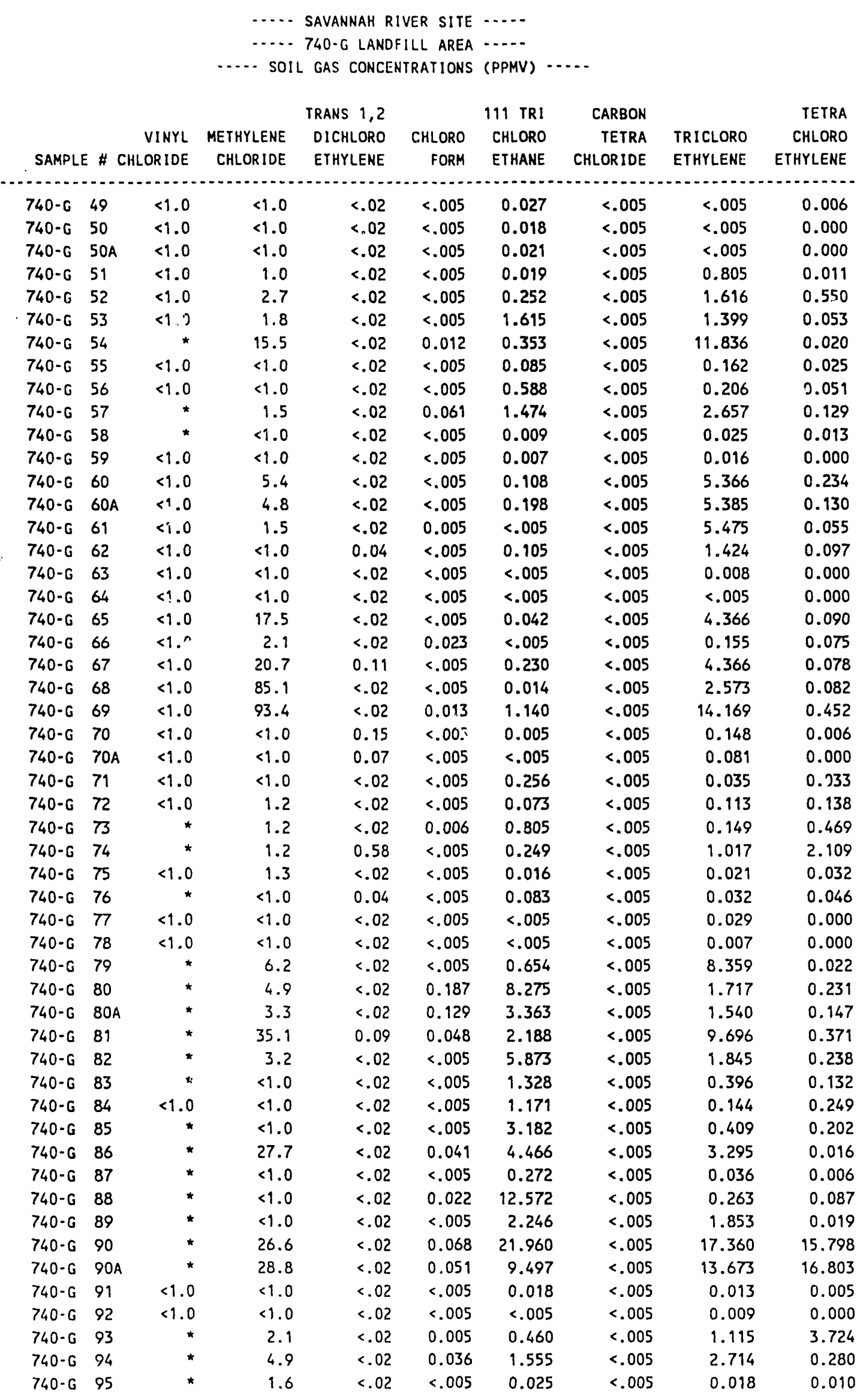

* SEE SECTION 111.0 .

Table 2. Chlorinated Hydrocarbon Soil Gas Concentrations and system Blanks at the 740-G Sanitary Landfill 


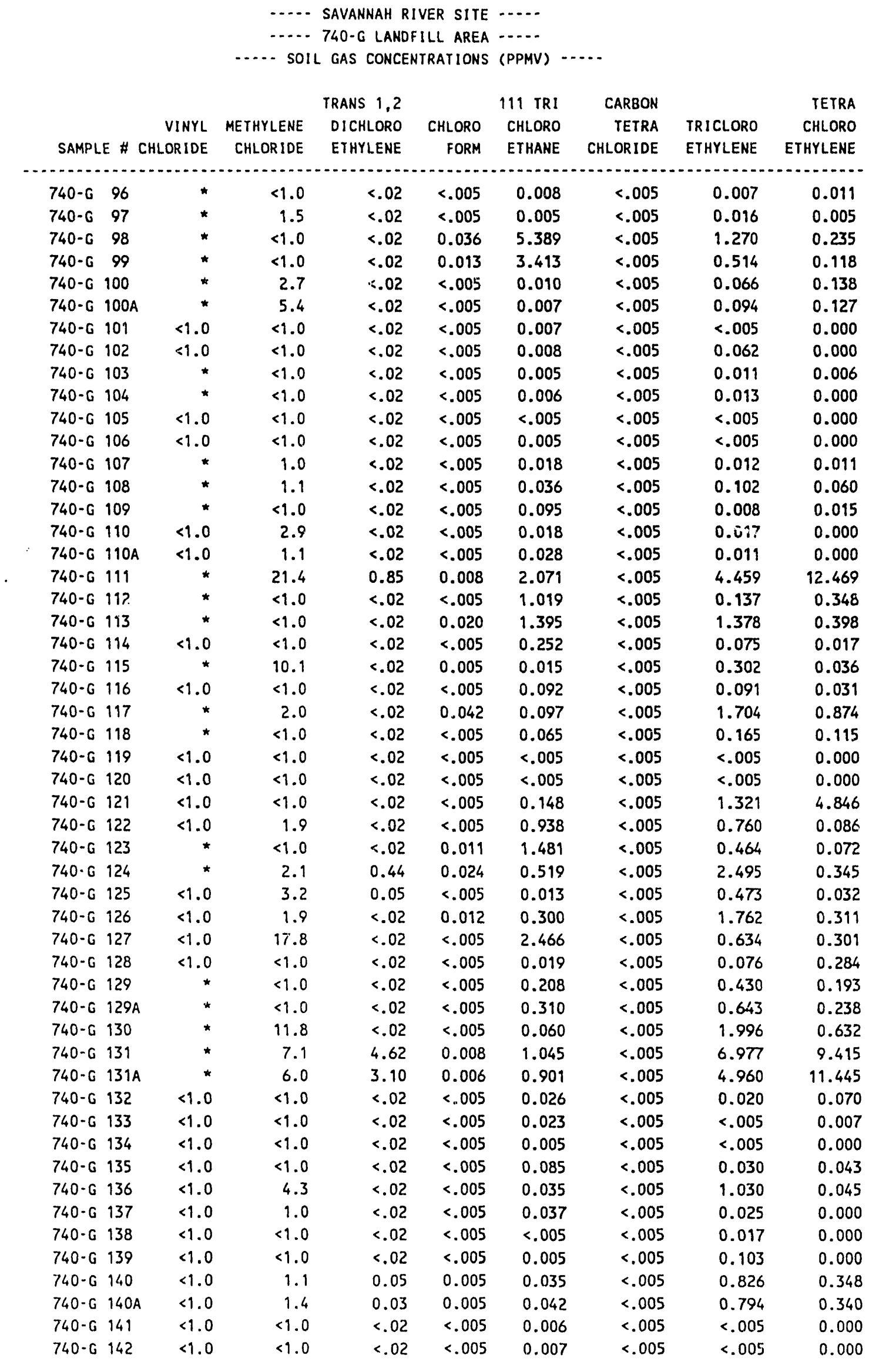

* SEE SECTION III. D.

Table 2. Chlorinated Hydrocarbon Soil Gas Concentrations and System Blanks at the 740-G Sanitary Landfill 
SAVANNAH RIVER SITE

.... 740-G LANOFILL AREA ....

.... SOIL GAS CONCENTRATIOHS (PPMV) .....

\begin{tabular}{|c|c|c|c|c|c|c|c|}
\hline & & TRANS 1,2 & & $111 \mathrm{TRI}$ & CARBON & & TETRA \\
\hline VINYL & METHYLENE & DICHLORO & CHLORO & CHLORO & TETRA & TRICLORO & CHLORO \\
\hline$\because:$ JRIDE & CHLORIDE & ETHYLENE & FORM & ETHANE & CHLORIDE & ETHYLENE & ETHYLENE \\
\hline$\cdots$ & . & . & $\ldots \ldots$ & 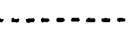 & - - - - - - & - & . \\
\hline$<1.0$ & $<1.0$ & $<.02$ & $<.005$ & 0.007 & $<.005$ & $<.005$ & 0.000 \\
\hline$<1.0$ & $<1.0$ & $<.02$ & $<.005$ & 0.005 & $<.005$ & $<.005$ & 0.000 \\
\hline$<1.0$ & $<1.0$ & $<.02$ & $<.005$ & $<.005$ & $<.005$ & $<.005$ & 0.000 \\
\hline$<1.0$ & $<1.0$ & $<.02$ & $<.005$ & 0.039 & $<.005$ & $<.005$ & 0.048 \\
\hline$<1.0$ & $<1.0$ & $<.02$ & $<.005$ & 0.007 & $<.005$ & $<.005$ & 0.000 \\
\hline$<1.0$ & $<1.0$ & $<.02$ & $<.005$ & 0.006 & $<.005$ & $<.005$ & 0.000 \\
\hline$<1.0$ & $<1.0$ & $<. C ?$ & $<.005$ & $<.005$ & $<.005$ & $<.005$ & 0.000 \\
\hline$<1.0$ & $<1.0$ & $<.02$ & $<.005$ & $<.005$ & $<.005$ & $<.005$ & 0.000 \\
\hline$<1.0$ & $<1.0$ & $<.02$ & $<.005$ & $<.005$ & $<.005$ & $<.005$ & 0.000 \\
\hline$<1.0$ & $<1.0$ & $<.02$ & $<.005$ & $<.005$ & $<.005$ & $<.005$ & 0.000 \\
\hline$<1.0$ & $<1.0$ & $<.02$ & $<.005$ & $<.005$ & $<.005$ & $<.005$ & 0.000 \\
\hline$<1.0$ & $<1.0$ & $<.02$ & $<.005$ & $<.005$ & $<.005$ & $<.005$ & 0.000 \\
\hline$<1.0$ & $<1.0$ & $<.02$ & $<.005$ & $<.005$ & $<.005$ & $<.005$ & 0.009 \\
\hline$<1.0$ & $<1.0$ & $<.02$ & $<.005$ & $<.005$ & $<.005$ & $<.005$ & $0.0 \mathrm{C} 0$ \\
\hline$<1.7$ & $<1.0$ & $<.02$ & $<.005$ & 0.224 & $<.005$ & $<.005$ & $0.02 s$ \\
\hline$\star$ & $<1.0$ & $<.02$ & 0.012 & 20.730 & 0.005 & 0.124 & 0.110 \\
\hline$<1.0$ & $<1.0$ & $<.02$ & $<.005$ & 1.417 & $<.005$ & 0.024 & 0.024 \\
\hline * & $<1.0$ & $<.02$ & 0.064 & 10.719 & 0.225 & 0.028 & 0.031 \\
\hline$<1.0$ & $<1.0$ & $<.02$ & $<.005$ & 0.861 & $<.005$ & $<.005$ & 0.005 \\
\hline$<1.0$ & $<1.0$ & $<.02$ & $<.005$ & 1.375 & $<.005$ & $<.005$ & 0.008 \\
\hline$<1.0$ & $<1.0$ & $=.02$ & $<.005$ & 0.260 & $<.005$ & $<.005$ & 0.000 \\
\hline$<1.0$ & $<1.0$ & $<.02$ & $<.005$ & 1.712 & $<.005$ & 0.011 & 0.040 \\
\hline$\star$ & $<1.0$ & $<.02$ & 0.033 & 18.561 & $<.005$ & 1.404 & 0.000 \\
\hline$\star$ & $<1.0$ & $<.02$ & $<.005$ & 2.932 & $<.005$ & 0.082 & 0.089 \\
\hline$\star$ & $<1.0$ & $<.02$ & $<.005$ & 1.336 & $<.005$ & $<.005$ & 0.000 \\
\hline$\therefore 1.0$ & $<1.0$ & $<.02$ & $<.005$ & 0.239 & $<.005$ & $<.005$ & 0.014 \\
\hline$<1.0$ & $<1.0$ & $<.02$ & $<.005$ & 0.195 & $<.005$ & $<.005$ & 0.006 \\
\hline$<1.0$ & $<1.0$ & $<.02$ & $<.005$ & 0.005 & $<.005$ & $<.005$ & 0.000 \\
\hline$<1.0$ & $<1.0$ & $<.02$ & $<.005$ & $<.005$ & $<.005$ & $<.005$ & 0.000 \\
\hline$<1.0$ & $<1.0$ & $<.02$ & $<.005$ & 0.632 & $<.005$ & $<.005$ & 0.037 \\
\hline$<1.0$ & $<1.0$ & $<.02$ & $<.005$ & 0.718 & $<.005$ & $<.005$ & 0.044 \\
\hline$\star$ & 1.6 & $<.02$ & 0.008 & 7.164 & $<.005$ & 1.517 & 0.653 \\
\hline * & 6.0 & $<.02$ & 0.014 & 2.432 & $<.005$ & 1.698 & 0.140 \\
\hline$\star$ & $<1.0$ & $<.02$ & 0.009 & 1.070 & $<.005$ & 0.327 & 0.010 \\
\hline ^ & $<1.0$ & $<.02$ & $<.005$ & 0.900 & $<.005$ & 0.048 & 0.006 \\
\hline$<1.0$ & 1.6 & $<.02$ & 0.203 & 112.378 & $<.005$ & 4.483 & 0.368 \\
\hline$\star$ & $<1.0$ & $<.02$ & 0.064 & 5.680 & $<.005$ & 0.189 & 0.462 \\
\hline$\star$ & 1.1 & $<.02$ & 0.011 & 4922 & $<.005$ & 0.501 & 0.050 \\
\hline$<9.0$ & $<1.0$ & $<.02$ & $<.005$ & 0.212 & $<.005$ & 0.091 & 0.000 \\
\hline * & 3.2 & $<.02$ & 0.020 & 2.118 & $<.005$ & 0.196 & 0.023 \\
\hline * & 3.6 & $<.02$ & 0.007 & 0.448 & $<.005$ & 0.289 & 0.011 \\
\hline$\star$ & 1.3 & $<.02$ & $<.005$ & $0.6: 0$ & $<.005$ & 0.071 & 0.000 \\
\hline * & $<1.0$ & $<.02$ & 0.011 & $16.82 i$ & $<.005$ & 1.416 & 0.195 \\
\hline$<1.0$ & $<1.0$ & $<.02$ & $<.005$ & $<.005$ & $<.005$ & $<.005$ & 0.000 \\
\hline$<1.0$ & $<1.0$ & $<.02$ & $<.005$ & $<.005$ & $<.005$ & $<.005$ & 0.000 \\
\hline * & $<1.0$ & $<.02$ & 0.054 & 11.853 & $<.005$ & 3.681 & 0.084 \\
\hline$\star$ & 1.3 & $<.02$ & 0.006 & 1.788 & $<.005$ & 0.733 & 0.067 \\
\hline$\star$ & $<1.0$ & $<.02$ & $<.005$ & 1.634 & $<.005$ & 0.330 & 0.021 \\
\hline * & 6.2 & $<.02$ & 0.045 & 17.309 & $<.005$ & 1.250 & 0.120 \\
\hline * & 1.4 & $<.02$ & 0.018 & 7.386 & 5.005 & 2.696 & 0.064 \\
\hline$\star$ & $<1.0$ & $<.02$ & 0.020 & 9.895 & $<.005$ & 0.575 & 0.555 \\
\hline$\star$ & $<1.0$ & $<.02$ & 0.008 & 7.095 & $<.005$ & 0.670 & 0.743 \\
\hline
\end{tabular}




\begin{tabular}{|c|c|c|c|c|c|c|c|c|}
\hline \multicolumn{9}{|c|}{$\begin{array}{l}\ldots . . . \\
\ldots \text { SAVANNAH RIVER SITE } \\
\ldots \text { SOIL GAS CONCENTRATIONS (PPMV) }\end{array}$} \\
\hline SÁMPLE \#C & $\begin{array}{l}\text { VINYL } \\
\text { LORIDE }\end{array}$ & $\begin{array}{l}\text { METHYLENE } \\
\text { CHLORIDE }\end{array}$ & $\begin{array}{l}\text { TRANS } 1,2 \\
\text { DICHLORO } \\
\text { ETHYLENE }\end{array}$ & $\begin{array}{r}\text { CHLORO } \\
\text { FORM }\end{array}$ & $\begin{array}{l}111 \text { TRI } \\
\text { CHLORO } \\
\text { ETHANE }\end{array}$ & $\begin{array}{r}\text { CARBON } \\
\text { TETRA } \\
\text { CHLORIDE }\end{array}$ & $\begin{array}{l}\text { TRICLORO } \\
\text { ETHYLENE }\end{array}$ & $\begin{array}{r}\text { TETRA } \\
\text { CHLORO } \\
\text { ETHYLENE }\end{array}$ \\
\hline $740-\mathrm{G} \quad 190 \mathrm{~A}$ & $\star$ & $<1.0$ & $<.02$ & 0.008 & 6.810 & $<.005$ & 0.759 & 0.888 \\
\hline $740-G \quad 191$ & * & 2.3 & $<.02$ & 0.013 & 6.018 & $<.005$ & 0.459 & 0.148 \\
\hline $740-G \quad 192$ & * & $<1.0$ & $<.02$ & 0.009 & 3.707 & $<.005$ & 0.162 & 0.074 \\
\hline $740-6 \quad 193$ & * & 2.1 & $<.02$ & $<.005$ & 0.025 & $<.005$ & $<.005$ & 0.000 \\
\hline $740-G 194$ & $<1.0$ & $<1.0$ & $<.02$ & $<.005$ & 0.031 & $<.005$ & $<.005$ & 0.000 \\
\hline $740-G \quad 195$ & $\star$ & $<1.0$ & $<.02$ & $<.005$ & 1.381 & $<.005$ & 0.069 & 0.052 \\
\hline $740-G 196$ & * & 1.1 & $<.02$ & $<.005$ & 0.006 & $<.005$ & $<.005$ & 0.000 \\
\hline $740-G \quad 197$ & $<1.0$ & 1.4 & $<.02$ & $<.005$ & $<.005$ & $<.005$ & $<.005$ & 0.000 \\
\hline $740-G \quad 198$ & $<1.0$ & $<1.0$ & $<.02$ & $<.005$ & 0.301 & $<.005$ & $<.005$ & 0.000 \\
\hline $740-G 199$ & * & 14.8 & $<.02$ & 0.206 & 27.223 & $<.005$ & 1.064 & 1.014 \\
\hline $740-G \quad 200$ & * & 1.0 & $<.02$ & $<.005$ & 2.746 & $<.005$ & 0.149 & 0.014 \\
\hline $740-G \quad 200 \mathrm{~A}$ & * & $<1.0$ & $<.02$ & $<.005$ & 2.504 & $<.005$ & 0.243 & 0.036 \\
\hline $740-6 \quad 201$ & $\star$ & 2.2 & $<.02$ & 0.177 & 35.930 & $<.005$ & 1.364 & 0.111 \\
\hline $740-G \quad 202$ & * & $<1.0$ & $<.02$ & 0.080 & 13.172 & $<.005$ & 0.964 & 0.190 \\
\hline $740-G \quad 203$ & $<1.0$ & $<1.0$ & $<.02$ & $<.005$ & 2.319 & $<.005$ & 0.023 & 0.034 \\
\hline $740-6204$ & * & $<1.0$ & $<.02$ & $<.005$ & 2.784 & $<.005$ & 0.008 & 0.016 \\
\hline $740-6205$ & * & 2.0 & $<.02$ & 0.085 & 16.496 & $<.005$ & 1.110 & 0.601 \\
\hline $740-G 206$ & * & $<1.0$ & $<.02$ & 0.007 & 21.770 & $<.005$ & 1.359 & 0.670 \\
\hline $740-6207$ & $<1.0$ & $<1.0$ & $<.02$ & $<.005$ & 0.031 & $<.005$ & 0.010 & 0.009 \\
\hline $740-G \quad 208$ & * & 39.2 & $<.02$ & 0.100 & 0.711 & $<.005$ & 0.876 & 0.094 \\
\hline $740-G \quad 209$ & * & $<1.0$ & $<.02$ & 0.071 & 10.508 & $<.005$ & 4.925 & 0.134 \\
\hline $740-G \quad 210$ & $<1.0$ & $<1.0$ & $<.02$ & $<.005$ & $<.005$ & $<.005$ & $<.005$ & 0.000 \\
\hline $740-G \quad 210 \mathrm{~A}$ & $<1.0$ & $<1.0$ & $<.02$ & $<.005$ & $<.005$ & $<.005$ & $<.005$ & 0.000 \\
\hline $740-0211$ & $<1.0$ & $<1.0$ & $<.02$ & $<.005$ & $<.005$ & $<.005$ & $<.005$ & 0.000 \\
\hline $740-G \quad 212$ & $<1.0$ & $<1.0$ & $<.02$ & 0.011 & 16.930 & $<.005$ & 0.694 & 0.751 \\
\hline $740-G \quad 213$ & $<1.0$ & $<1.0$ & $<.02$ & 0.011 & 10.281 & $<.005$ & $<.005$ & 0.016 \\
\hline $740-G \quad 214$ & * & 4.9 & $<.02$ & 0.022 & 25.101 & $<.005$ & 0.298 & 0.264 \\
\hline $740-G 215$ & * & 1.5 & $<.02$ & 0.006 & 2.731 & $<.005$ & $<.005$ & 0.023 \\
\hline $740-6216$ & * & $<1.0$ & $<.02$ & $<.005$ & 57.413 & $<.005$ & 0.044 & 0.010 \\
\hline $740-6 \quad 217$ & $<1.0$ & $<1.0$ & $<.02$ & $<.005$ & 2.913 & $<.005$ & $<.005$ & 0.000 \\
\hline $740-G 218$ & $<1.0$ & $<1.0$ & $<.02$ & $<.005$ & 6.615 & $<.005$ & $<.005$ & 0.000 \\
\hline $740-G \quad 219$ & * & $<1.0$ & $<.02$ & $<.005$ & 9.182 & $<.005$ & $<.005$ & 0.009 \\
\hline $740-6220$ & * & $<1.0$ & $<.02$ & $<.005$ & 0.023 & $<.005$ & 0.065 & 0.000 \\
\hline $740-G 220 A$ & $\star$ & $<1.0$ & $<.02$ & $<.005$ & 0.024 & $<.005$ & 0.006 & 0.000 \\
\hline $740-G 221$ & $\star$ & 1.3 & $<.02$ & 0.008 & 0.339 & $<.005$ & $<.005$ & 0.032 \\
\hline $740-6222$ & * & $<1.0$ & $<.02$ & 0.005 & 0.104 & $<.005$ & $<.005$ & 0.000 \\
\hline $740-G 223$ & $<1.0$ & $<1.0$ & $<.02$ & $<.005$ & 0.045 & $<.005$ & $<.005$ & 0.000 \\
\hline $740-G 224$ & $<1.0$ & $<1.0$ & $<.02$ & $<.005$ & $<.005$ & $<.005$ & $<.005$ & 0.000 \\
\hline $740-6225$ & $<1.0$ & $<1.0$ & $<.02$ & 0.010 & $<.005$ & $<.005$ & $<.005$ & 0.000 \\
\hline $740-6226$ & $<1.0$ & 1.3 & $<.02$ & $<.005$ & 0.010 & $<.005$ & $<.005$ & 0.000 \\
\hline $740-6227$ & $<1.0$ & 1.3 & $<.02$ & $<.005$ & 3.129 & $<.005$ & 0.020 & 0.000 \\
\hline $740-G 228$ & * & 1.3 & $<.02$ & $<.005$ & 0.909 & $<.005$ & $<.005$ & 0.000 \\
\hline $740-G 229$ & $<1.0$ & 2.0 & $<.02$ & $<.005$ & 0.366 & $<.005$ & $<.005$ & 0.000 \\
\hline $740-G 230$ & $<1.0$ & 1.1 & $<.02$ & $<.005$ & 0.377 & 0.006 & $<.005$ & 0.000 \\
\hline $740-G 230 \mathrm{~A}$ & $<1.0$ & 1.4 & $<.02$ & $<.005$ & 0.540 & 0.007 & $<.005$ & 0.000 \\
\hline $740-G 231$ & $<1.0$ & 1.0 & $<.02$ & $<.005$ & 0.010 & $<.005$ & $<.005$ & 0.000 \\
\hline $740-G 232$ & * & 2.0 & $<.02$ & $<.005$ & $<.005$ & $<.005$ & $<.005$ & 0.000 \\
\hline $740-G 233$ & $<1.0$ & $<1.0$ & $<.02$ & $<.005$ & 0.143 & $<.005$ & $<.005$ & 0.000 \\
\hline $740-6234$ & $<1.0$ & $<1.0$ & $<.02$ & $<.005$ & 0.026 & $<.005$ & $<.005$ & 0.000 \\
\hline $740-G 235$ & $<1.0$ & $<1.0$ & $<.02$ & $<.005$ & 0.008 & $<.005$ & $<.005$ & 0.000 \\
\hline $740-G 236$ & $<1.0$ & $<1.0$ & $<.02$ & $<.005$ & 1.443 & $<.005$ & $<.005$ & 0.000 \\
\hline $740-6 \quad 237$ & * & $<1.0$ & $<.02$ & $<.005$ & 6.686 & $<.005$ & 0.041 & 0.054 \\
\hline
\end{tabular}

* SEE SECTION 111.0 .

Table 2. Chlorinated Hydrocarbon Soil Gas Concentrations and System Blanks at the 740-G Sanitary Landfill 


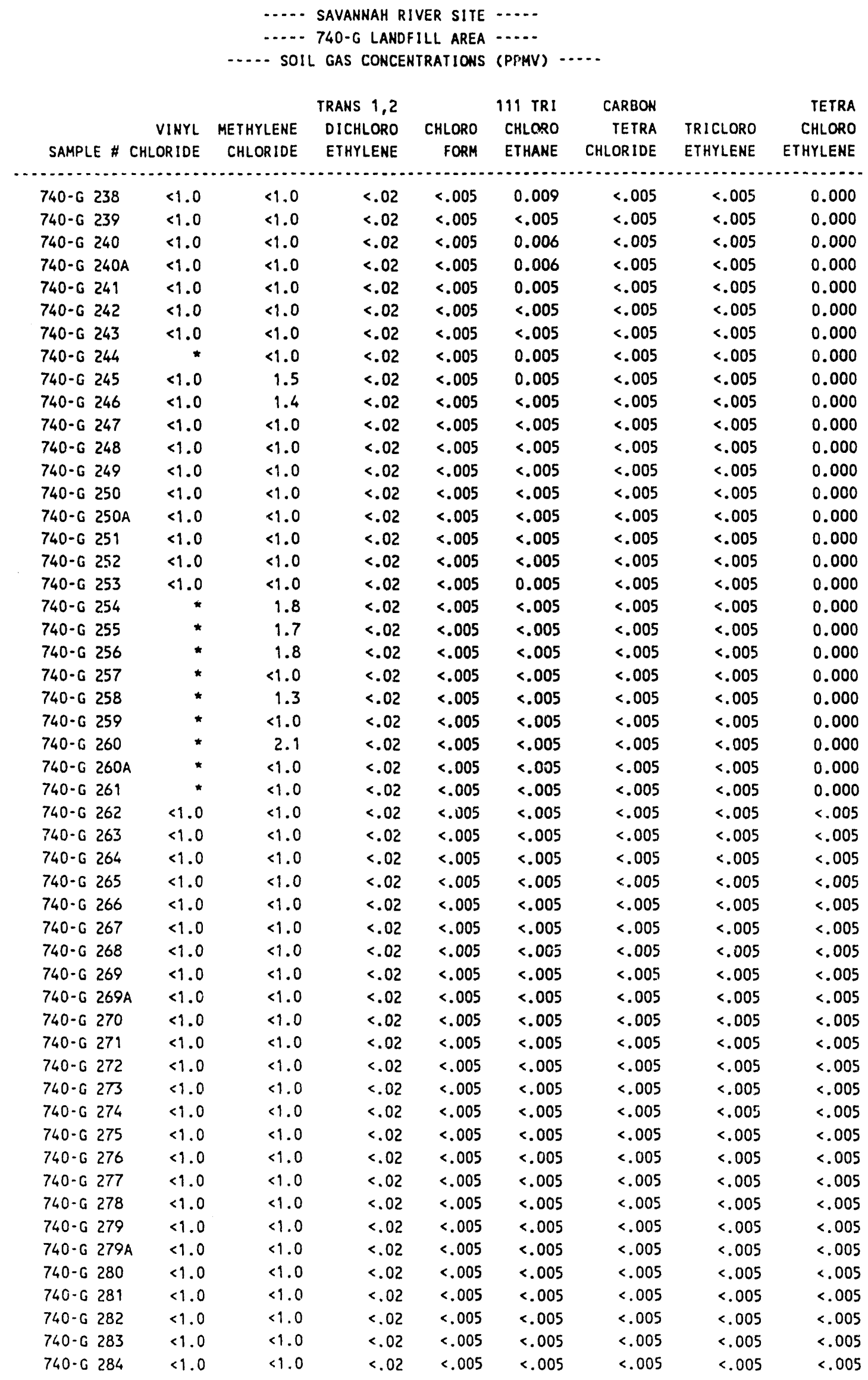

* SEe SECTION 111.0.

Table 2. Chlorinated Hydrocarbon Soil Gas Concentrations and System Blanks at the 740-G Sanitary Landfill 
-... SAVANNAH RIVER SITE ....

.... 740-G LANDFILL AREA .....

SOIL GAS CONCENTRATIONS (PPMV) .....

\begin{tabular}{|c|c|c|c|c|c|c|c|c|}
\hline SAMPLE \# & $\begin{array}{r}\text { VINYL } \\
\text { CHLORIDE }\end{array}$ & $\begin{array}{r}\text { METHYLENE } \\
\text { CHLORIDE }\end{array}$ & $\begin{array}{l}\text { TRANS } 1,2 \\
\text { DICHLORO } \\
\text { ETHYLENE }\end{array}$ & $\begin{array}{r}\text { CHLORO } \\
\text { FORM }\end{array}$ & $\begin{array}{l}111 \text { TRI } \\
\text { CHLORO } \\
\text { ETHANE }\end{array}$ & $\begin{array}{r}\text { CARBON } \\
\text { TETRA } \\
\text { CHLORIDE }\end{array}$ & $\begin{array}{l}\text { TRICLORO } \\
\text { ETHYLENE }\end{array}$ & $\begin{array}{r}\text { TETRA } \\
\text { CHLORO } \\
\text { ETHYLENE }\end{array}$ \\
\hline 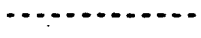 & & & & & & & & \\
\hline $740-6285$ & $<1.0$ & $<1.0$ & $<.02$ & $<.005$ & .007 & $<.005$ & $<.005$ & $<.005$ \\
\hline $740-G 286$ & $<9.0$ & $<1.0$ & $<.02$ & $<.005$ & $<.005$ & $<.005$ & $<.005$ & $<.005$ \\
\hline $740-G \quad 287$ & $<1.0$ & $<1.0$ & $<.02$ & $<.005$ & $<.005$ & $<.005$ & $<.005$ & $<.005$ \\
\hline $740-G 288$ & $<i .0$ & $<1.0$ & $<.02$ & $<.005$ & $<.005$ & $<.005$ & $<.005$ & $<.005$ \\
\hline 740-G BLK 1 & $<1.0$ & $<1.0$ & $<.02$ & $<.005$ & $<.005$ & $<.005$ & $<.005$ & $<.005$ \\
\hline $740-G$ BLK 2 & $<1.0$ & $<1.0$ & $<.02$ & $<.005$ & $<.005$ & $<.005$ & $<.005$ & $<.005$ \\
\hline $740-G$ BLK & $<1.0$ & $<1.0$ & $<.02$ & $<.005$ & 0.007 & $<.005$ & $<.005$ & $<.005$ \\
\hline 740-G BLK $l$, & $<1.0$ & $<1.0$ & $<.02$ & $<.005$ & $<.005$ & $<.005$ & 0.010 & 0.013 \\
\hline $740-G$ BLK & $<1.0$ & $<1.0$ & $<.02$ & $<.005$ & 0.009 & $<.005$ & $<.005$ & $<.005$ \\
\hline 740-G BLK 6 & $<1.0$ & $<9.0$ & $<.02$ & $<.005$ & $<.005$ & $<.005$ & $<.005$ & $<.005$ \\
\hline 740-G BLK 7 & $<1.0$ & $<1.0$ & $<.02$ & $<.005$ & 0.005 & $<.005$ & 0.019 & $<.005$ \\
\hline 740-G BLK 8 & $<1.0$ & $<1.0$ & $<.02$ & $<.005$ & $<.005$ & $<.005$ & $<.005$ & $<.005$ \\
\hline 740-G BLK 9 & $<1.0$ & $<1.0$ & $<.02$ & $<.005$ & 0.005 & $<.005$ & $<.005$ & $<.005$ \\
\hline $740-G$ BLK. 10 & $<1.0$ & $<1.0$ & $<.02$ & $<.005$ & $<.005$ & $<.005$ & 0.039 & $<.005$ \\
\hline $740-G$ BLK 11 & $<1.0$ & $<1.0$ & $<.02$ & $<.005$ & 0.011 & $<.005$ & 0.044 & 0.016 \\
\hline 740-G BLK 12 & $<1.0$ & $<1.0$ & $<.02$ & $<.005$ & $<.005$ & $<.005$ & $<.005$ & $<.005$ \\
\hline $740-6$ BLK 13 & $<1.0$ & $<1.0$ & $<.02$ & $<.005$ & $<.005$ & $<.005$ & $<.005$ & $<.005$ \\
\hline $740 . G$ BLK 14 & $<1.0$ & $<1.0$ & $<.02$ & $<.005$ & 0.006 & $<.005$ & 0.022 & 0.053 \\
\hline $740-G$ BLK 15 & $<1.0$ & $<1.0$ & $<.02$ & $<.005$ & 0.005 & $<.005$ & $<.005$ & 0.053 \\
\hline $740-C$ BLK 16 & $<1.0$ & $<1.0$ & $<.02$ & $<.005$ & $<.005$ & $<.005$ & $<.005$ & $<.005$ \\
\hline 740-G BLK 17 & $<1.0$ & $<1.0$ & $<.02$ & $<.005$ & 0.006 & $<.005$ & $<.005$ & $<.005$ \\
\hline $740-G$ BLK 18 & $<1.0$ & $<1.0$ & $<.02$ & $<.005$ & $<.005$ & $<.005$ & $<.005$ & $<.005$ \\
\hline 740-C BLK 19 & $<1.0$ & $<1.0$ & $<.02$ & $<.005$ & $<.005$ & $<.005$ & $<.005$ & $<.005$ \\
\hline $740-G$ BLK 20 & $<9.0$ & $<1.0$ & $<.02$ & $<.005$ & 0.023 & $<.005$ & $<.005$ & $<.005$ \\
\hline $740-G$ BLK 21 & $<1.0$ & $<1.0$ & $<.02$ & $<.005$ & $<.005$ & $<.005$ & $<.005$ & $<.005$ \\
\hline $740-G$ BLK 22 & $<1.0$ & $<1.0$ & $<.02$ & $<.005$ & 0.010 & $<.005$ & $<.005$ & 0.006 \\
\hline $740-G$ BLK 23 & $<1.0$ & $<1.0$ & $<.02$ & $<.005$ & 0.005 & $<.005$ & $<.005$ & $<.005$ \\
\hline $740-G$ BLK 24 & $<1.0$ & $<1.0$ & $<.02$ & $<.005$ & $<.005$ & $<.005$ & $<.005$ & $<.005$ \\
\hline $740-G$ BLK 25 & $<1.0$ & $<1.0$ & $<.02$ & $<.005$ & $<.005$ & $<.005$ & $<.005$ & $<.005$ \\
\hline $740-G$ BLK 26 & $<1.0$ & $<1.0$ & $<.02$ & $<.005$ & $<.005$ & $<.005$ & $<.005$ & $<.005$ \\
\hline $740-G$ BLK 27 & $<1.0$ & $<1.0$ & $<.02$ & $<.005$ & 0.005 & $<.005$ & $<.005$ & $<.005$ \\
\hline $740-G$ BLK 28 & $<1.0$ & $<1.0$ & $<.02$ & $<.005$ & $<.005$ & $<.005$ & $<.005$ & $<.005$ \\
\hline $740-G$ BLK 29 & * & $<1.0$ & $<.02$ & $<.005$ & $<.005$ & $<.005$ & $<.005$ & $<.005$ \\
\hline $740-G$ BLK 30 & * & $<1.0$ & $<.02$ & $<.005$ & $<.005$ & $<.005$ & $<.005$ & $<.005$ \\
\hline $740-G$ BLK 31 & $<1.0$ & $<1.0$ & $<.02$ & $<.005$ & $<.005$ & $<.005$ & $<.005$ & $<.005$ \\
\hline $740-G$ BLK 32 & $<1.0$ & $<1.0$ & 0.03 & $<.005$ & $<.005$ & $<.005$ & $<.005$ & $<.005$ \\
\hline $740-6$ BLK 33 & $<1.0$ & $<1.0$ & 0.03 & $<.005$ & $<.005$ & $<.005$ & $<.005$ & $<.005$ \\
\hline $740-G$ BLK 34 & $<1.0$ & $<1.0$ & $<.02$ & $<.005$ & $<.005$ & $<.005$ & $<.005$ & $<.005$ \\
\hline
\end{tabular}

* SEE SECTIOH: III. D.

Table 2. Chlorinated Hydrocarbon Soil Gas Concentrations and System Blanks at the 740-G Sanitary Landfill 


\section{This page intentionally left blank.}


... 740-G LANDFILL -...

...... SOIL GAS AND MERCURY CONCENTRATIONS a 3 FT-.....

\begin{tabular}{|c|c|c|c|c|c|c|c|c|c|c|c|c|}
\hline $\begin{array}{c}\text { SAMPLE } \\
\#\end{array}$ & & $\begin{array}{r}\text { METHANE } \\
\%\end{array}$ & $\begin{array}{r}\text { METHANE } \\
\text { PPB }\end{array}$ & $\begin{array}{r}\text { ETHANE } \\
\text { PPB }\end{array}$ & $\begin{array}{r}\text { PROPANE } \\
\text { PPB }\end{array}$ & $\begin{array}{r}\text { I-BUTANE } \\
\text { PPB }\end{array}$ & $\begin{array}{c}\text { N-BUTANE } \\
\text { PPB }\end{array}$ & $\begin{array}{c}\text { ETHYLENE } \\
\text { PPB }\end{array}$ & $\begin{array}{c}\text { PROPYLENE } \\
\text { PPB }\end{array}$ & $\begin{array}{r}\text { MERCURY } \\
\mathrm{ng} / \mathrm{g}\end{array}$ & $\begin{array}{c}\text { SAMPLE } \\
\#\end{array}$ & \\
\hline $740-G$ & 1 & 16.4 & 163880000 & $<5$ & 8696 & 10532 & 1992 & 1083 & 1687 & 1 & $740-G$ & 1 \\
\hline $740-6$ & 2 & 24.0 & 240120000 & $<5$ & 8634 & 13757 & $<5$ & 376 & 246 & $<1$ & $740-G$ & 2 \\
\hline $740-6$ & 3 & 25.6 & 256050000 & $<5$ & 2860 & 5170 & $<5$ & 1500 & 917 & 2 & $740-G$ & 3 \\
\hline $740-6$ & 4 & 48.8 & 487760000 & $<5$ & 797 & 295 & $<5$ & $<5$ & 317 & $<1$ & $740-G$ & 4 \\
\hline $740-6$ & 5 & 40.0 & 399640000 & $<5$ & 56 & $<5$ & $<5$ & $<5$ & $<5$ & 2 & $740-G$ & 5 \\
\hline $740-G$ & 6 & 9.2 & 92353000 & $<5$ & 1029 & 603 & 118 & 541 & 299 & $<1$ & $740-G$ & 6 \\
\hline $740-G$ & 7 & 0.0 & 4069 & 47 & 15 & $<5$ & $<5$ & 25 & 60 & $<1$ & $740-G$ & 7 \\
\hline $740-G$ & 8 & 0.0 & 2902 & 25 & 9 & $<5$ & $<5$ & 15 & 112 & 2 & $740-6$ & 8 \\
\hline $740-6$ & 9 & 46.2 & 461820000 & $<5$ & 8518 & 12590 & $<5$ & $<5$ & 938 & 33 & $740-6$ & 9 \\
\hline $740-G$ & 10 & 52.9 & 528570000 & $<5$ & 2682 & 2528 & 216 & 3139 & 1814 & 6 & $740-G$ & 10 \\
\hline $740-6$ & $10 \mathrm{~A}$ & 53.2 & 532080000 & $<5$ & 2173 & 919 & $<5$ & 3091 & 808 & 5 & $740-6$ & $10 A$ \\
\hline $740-6$ & 11 & 61.0 & 610080000 & $<5$ & 12461 & 19042 & 260 & 2088 & 2927 & 6 & $740-G$ & 11 \\
\hline $740-G$ & 12 & 58.1 & 581080000 & $<5$ & 17860 & 22009 & $<5$ & 1864 & 3220 & 5 & $740-G$ & 12 \\
\hline $740-G$ & 13 & 50.3 & 503050000 & $<5$ & 20996 & 26054 & $<5$ & 1526 & 9241 & 9 & $740-6$ & 13 \\
\hline $740-6$ & 14 & 0.0 & 394117 & 88 & 218 & 535 & $<5$ & 42 & 758 & 3 & $740-G$ & 14 \\
\hline $740-6$ & 15 & 40.6 & 405560000 & $<5$ & 9852 & 10889 & 352 & 2406 & 4159 & 5 & $740-6$ & 15 \\
\hline $740-G$ & 16 & 57.4 & 573780000 & 1858 & 2924 & $<5$ & $<5$ & 7250 & 7852 & 9 & $740-G$ & 16 \\
\hline $740-G$ & 17 & 58.1 & 581080000 & $<5$ & 12808 & 12891 & $<5$ & $<5$ & 1479 & 2 & $740-G$ & 17 \\
\hline $740-G$ & 18 & 58.1 & 580820000 & $<5$ & 9269 & 14401 & $<5$ & 2061 & 2494 & 5 & $740-G$ & 18 \\
\hline $740-6$ & 19 & 55.7 & 557210000 & $<5$ & 959 & 151 & $<5$ & 936 & 1801 & 12 & $740-6$ & 19 \\
\hline $740-G$ & 20 & 44.7 & 446560000 & $<5$ & 875 & $<5$ & $<5$ & $<5$ & 398 & 3 & $740-G$ & 20 \\
\hline $740-G$ & $20 \mathrm{~A}$ & 54.6 & 546460000 & $<5$ & 3035 & 459 & 187 & $<5$ & 163 & 5 & $740-G$ & $20 \mathrm{~A}$ \\
\hline $740-G$ & 21 & 0.0 & 5586 & 38 & 50 & 13 & $<5$ & 24 & 195 & 3 & $740-G$ & 21 \\
\hline $740-G$ & 22 & 0.0 & 11893 & 95 & 66 & 464 & 6 & 53 & 231 & 2 & $740-G$ & 22 \\
\hline $740-G$ & 23 & 62.7 & 627470000 & $<5$ & 30152 & 28918 & $<5$ & 3360 & 5918 & 5 & $740-G$ & 23 \\
\hline $740-G$ & 24 & 60.9 & 608600000 & $<5$ & 7272 & 4919 & $<5$ & 1446 & 2070 & 7 & $740-G$ & 24 \\
\hline $740-G$ & 25 & 49.4 & 493720000 & $<5$ & 6547 & 5037 & $<5$ & 4082 & 3367 & 11 & $740-6$ & 25 \\
\hline $740-G$ & 26 & 8.8 & 87769800 & $<5$ & 671 & $<5$ & $<5$ & 739 & 378 & 10 & $740-6$ & 26 \\
\hline $740-G$ & 27 & 46.3 & 462890000 & $<5$ & 3881 & 4452 & $<5$ & $<5$ & 1191 & 1 & $740-6$ & 27 \\
\hline $740-G$ & 28 & 32.5 & 325210000 & $<5$ & 6297 & 6486 & $<5$ & 2004 & 3649 & 6 & $740-6$ & 28 \\
\hline $740-G$ & 29 & 52.1 & 520610000 & $<5$ & 2390 & 2320 & $<5$ & 2135 & 918 & 5 & $740-6$ & 29 \\
\hline $740-6$ & 30 & 50.8 & 508010000 & $<5$ & 33031 & 52445 & 1331 & 7102 & 27073 & $<1$ & $740-G$ & 30 \\
\hline $740-6$ & $30 \mathrm{~A}$ & 53.1 & 530910000 & $<5$ & 39640 & 61157 & 1417 & 7923 & 34926 & 2 & $740-6$ & $30 \mathrm{~A}$ \\
\hline $740-G$ & 31 & 1.6 & 15827338 & $<5$ & 1528 & 230 & $<5$ & 917 & $<5$ & 2 & $740-6$ & 31 \\
\hline $740-G$ & 32 & 49.4 & 493690000 & $<5$ & 4964 & 7435 & $<5$ & 895 & 1620 & 2 & $740-G$ & 32 \\
\hline $740-G$ & 33 & 11.6 & 115827000 & $<5$ & 1091 & 434 & $<5$ & 485 & 274 & 3 & $740-G$ & 33 \\
\hline $740-G$ & 34 & 41.4 & 414010000 & $<5$ & 253 & 334 & 267 & 3176 & 33961 & 3 & $740-6$ & 34 \\
\hline $740-G$ & 35 & 0.0 & 6736 & 24 & 10 & $<5$ & $<5$ & 18 & 1376 & 3 & $740-G$ & 35 \\
\hline $740-0$ & 36 & 0.0 & 3054 & 23 & 10 & $<5$ & $<5$ & 14 & 51 & 1 & $740-G$ & 36 \\
\hline $740-6$ & 37 & 25.6 & 256140000 & $<5$ & 2515 & 445 & $<5$ & $<5$ & 671 & 2 & $740-G$ & 37 \\
\hline $740-G$ & 30 & 4.2 & 41726600 & $<5$ & $<5$ & $<5$ & $<5$ & $<5$ & $<5$ & $<1$ & $740-6$ & 38 \\
\hline $740-6$ & 39 & 7.4 & 74244600 & $<5$ & 5870 & 6767 & 299 & $<5$ & $<5$ & 3 & $740-6$ & 39 \\
\hline $740-G$ & 40 & 19.1 & 191130000 & $<5$ & 1644 & 3915 & $<5$ & $<5$ & $<5$ & 2 & $740-G$ & 40 \\
\hline $740-G$ & $40 A$ & 16.1 & 160630000 & $<5$ & 2074 & 3882 & $<5$ & 493 & 604 & 3 & $740-6$ & $40 \mathrm{~A}$ \\
\hline $740-G$ & 41 & 52.3 & 523400000 & $<5$ & 65786 & 85546 & 2070 & 4445 & 11025 & 3 & $740-6$ & 41 \\
\hline $740-G$ & 42 & 35.7 & 357060000 & $<5$ & 26959 & 33780 & $<5$ & 1464 & 785 & 7 & $740-G$ & 42 \\
\hline $740-G$ & 43 & 33.0 & 329940000 & 7348 & 71537 & 45651 & 33045 & 7144 & 3617 & 2 & $740-6$ & 43 \\
\hline $740-G$ & 44 & 23.4 & 233660000 & $<5$ & 293 & $<5$ & $<5$ & $<5$ & $<5$ & $<1$ & $740-G$ & 44 \\
\hline $740-G$ & 45 & 32.5 & 324770000 & $<5$ & 161 & $<5$ & 75 & 774 & 485 & 2 & $740-G$ & 45 \\
\hline $740-G$ & 46 & 49.7 & 497180000 & $<5$ & 780 & 621 & $<5$ & 1249 & 1119 & 7 & $740-G$ & 46 \\
\hline $740-G$ & 47 & 46.0 & 459540000 & $<5$ & 6319 & 18454 & 4678 & 2057 & 1057 & 3 & $740-G$ & 47 \\
\hline $740-G$ & 48 & 49.5 & 495120000 & $<5$ & 517 & 1181 & $<5$ & $<5$ & 994 & 4 & $740-G$ & 48 \\
\hline $740-G$ & 49 & 0.0 & 11499 & 14 & 179 & 558 & $<5$ & 8 & 28 & 2 & $740-G$ & 49 \\
\hline
\end{tabular}

Table 3. Light Hydrocarbon Soil Gas, Soil Mercury Concentrations and System Blanks at the 740-G Sanitary Landfill 


\begin{tabular}{|c|c|c|c|c|c|c|c|c|c|c|c|c|}
\hline $\begin{array}{c}\text { SAMPLE } \\
\#\end{array}$ & & $\begin{array}{r}\text { METHANE } \\
\%\end{array}$ & $\begin{array}{r}\text { METHANE } \\
\text { PPB }\end{array}$ & $\begin{array}{r}\text { ETHANE } \\
\text { PPB }\end{array}$ & $\begin{array}{c}\text { PROPANE } \\
\text { PPB }\end{array}$ & $\begin{array}{c}\text { 1-BUTANE } \\
\text { PPB }\end{array}$ & $\begin{array}{c}\text { N-BUTANE E } \\
\text { PPB }\end{array}$ & $\begin{array}{c}\text { ETHYLENE } \\
\text { PPB }\end{array}$ & $\begin{array}{c}\text { PROPYLENE } \\
\text { PPB }\end{array}$ & $\begin{array}{r}\text { MERCURY } \\
\mathrm{ng} / \mathrm{s}\end{array}$ & $\begin{array}{c}A M L \\
7\end{array}$ & \\
\hline $740-G$ & 50 & 0.0 & 7248 & 12 & $<5$ & $<5$ & $<5$ & 6 & $\varepsilon$ & $?$ & $, \ldots, \quad \vdots$ & $\therefore$ \\
\hline $740-G$ & $50 \mathrm{~A}$ & 0.0 & 4717 & 11 & $<5$ & $<5$ & $<5$ & 6 & $i 2$ & $\vdots$ & $\ldots,-1, \quad:$ & $:$ \\
\hline $740-G$ & 51 & 50.5 & 505000000 & $<5$ & 744 & 49 & $<5$ & 1128 & 1657 & $t$ & $\therefore \cdots$ & $\because \cdot$ \\
\hline $740-G$ & 52 & 38.3 & 382810000 & $<5$ & 25926 & 34516 & 7929 & 2403 & $340:$ & $\because$ & $\therefore \therefore \cdot i$ & $\because$ \\
\hline $740-G$ & 53 & 27.7 & 277180000 & $<5$ & 19614 & 27170 & 3390 & 2191 & $56: 3$ & ‘ & $\therefore \cdots$ & \\
\hline $740-G$ & 54 & 19.5 & 195270000 & 7072 & 88749 & 180900 & 81352 & 5021 & $351 \%$ & 4 & $\therefore \quad \cdots$ & $\vdots$ \\
\hline $740-G$ & 55 & 15.5 & 154900000 & 5496 & 38646 & 68307 & 32640 & 1975 & 2486 & 4 & $\because \cdots$ & $\therefore$ \\
\hline $740-G$ & 56 & 0.0 & 4474 & 140 & 2056 & 7593 & 977 & 82 & $142 ?$ & 4 & , & 5 \\
\hline $740-G$ & 57 & 4.0 & 40287800 & 15719 & 75882 & 111690 & 49576 & 4195 & 13673 & $d$ & $7 \ldots$ & $:$ \\
\hline $740-G$ & 58 & 0.5 & 5366764 & 324 & 321 & 317 & 112 & 204 & $2 \leq 8$ & 4 & $\because \cdots \cdot$ & $\vdots$ \\
\hline $740-6$ & 59 & 0.0 & 7135 & 292 & 91 & 9 & 14 & 122 & $1 E T$ & $\vdots$ & $: \quad \quad$ & $!$ \\
\hline $740-G$ & 60 & 45.1 & 451280000 & 9864 & 108420 & 118110 & 45512 & 7000 & $91: 5$ & i & i & : \\
\hline $740-G$ & $60 \mathrm{~A}$ & 41.6 & 415990000 & 8725 & 92550 & 104680 & 39860 & 6258 & 975i & 3 & $\because \cdots$ & $!$ \\
\hline $740-G$ & 61 & 62.4 & 624150000 & 16095 & 58669 & 67522 & 32344 & 10206 & 9755 & $\because$ & $\vdots$ & \\
\hline $740-G$ & 62 & 38.4 & 383680000 & 9530 & 154460 & 154810 & 66065 & 5802 & 19716 & $?$ & & \\
\hline $740-G$ & 63 & 0.0 & 29418 & 7 & 15 & 10 & $<5$ & 6 & 5 & $\vdots$ & $;$ & \\
\hline $740-G$ & 64 & 0.0 & 4070 & 10 & 6 & $<5$ & $<5$ & 6 & $\varepsilon$ & $?$ & $\therefore \quad \ddots$ & \\
\hline $740-G$ & 65 & 43.6 & 435590000 & 24923 & 90543 & 113240 & 58836 & 14580 & $9527 i$ & $:$ & ; & \\
\hline $740-G$ & 66 & 62.4 & 624010000 & $<5$ & 8398 & 10338 & 8959 & 5422 & 504 & & & \\
\hline $740-6$ & 67 & 39.6 & 395930000 & 33989 & 84643 & 82375 & 43661 & 11458 & 12577 & 7 & $\because \cdots \cdot i$ & $\because \%$ \\
\hline $740-G$ & 68 & 37.9 & 378580000 & 42049 & 98591 & 122150 & 60789 & 20559 & $20850^{\circ}$ & 12. & $\dot{i} \quad-i$ & \\
\hline $740-G$ & 69 & 37.0 & 369620000 & 67735 & 86727 & 115140 & 84590 & 23396 & $218: 9$ & 10 & $i \cdot i_{i}$ & $\therefore$ \\
\hline $740-6$ & 70 & 34.6 & 346480000 & 13189 & 21580 & 20711 & 12412 & 7978 & 5524 & 10 & $\ldots \cdots$ & $\therefore$ \\
\hline $740-G$ & $70 A$ & 36.4 & 364070000 & 9817 & 14437 & 12836 & 7523 & 7038 & 4589 & $?$ & $\because \quad \cdots$ & 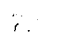 \\
\hline $740-G$ & 71 & 0.0 & 24547 & 62 & 49 & 74 & 9 & 38 & $1: 52$ & $\because$ & $\therefore \quad:$ & $; ;$ \\
\hline $740-G$ & $72 A$ & 13.8 & 138129490 & 20363 & 44207 & 18201 & 17242 & 3400 & $6 \div 02$ & 7 & $i \quad \cdots$ & \\
\hline $740-6$ & 73 & 13.9 & 138705000 & 52888 & 71803 & 37527 & $317 r 7$ & 7530 & 12559 & $t$ & $; \cdots$ & $\because$ \\
\hline $740-G$ & 74 & 16.3 & 163453200 & 77508 & 80894 & 65684 & 45918 & 19387 & 19110 & 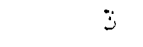 & $i \quad \cdots$ & $\because$ \\
\hline $740-G$ & 75 & 31.2 & 312260000 & 45465 & 110730 & 89467 & 65164 & 7622 & 5559 & $t$ & & $\therefore$ \\
\hline $740-G$ & 76 & 15.3 & 153230000 & 27359 & 97662 & 68734 & 55139 & 4026 & $587 i$ & $\angle$ & & $\because$ \\
\hline $740-G$ & $\pi$ & 0.0 & 13426 & 22 & 21 & 17 & $<5$ & 11 & is & 6 & $i \quad 6$ & 7 \\
\hline $740-G$ & 78 & 0.0 & 5655 & 15 & 12 & 7 & $<5$ & 9 & 17 & $\because$ & $i \cdots$ & $i$ \\
\hline $740-G$ & 79 & 24.4 & 244210000 & 101190 & 201140 & 273240 & 166320 & 19586 & 21157 & $i$ & & $\because:$ \\
\hline $740-G$ & 80 & 2.4 & 24172600 & 52391 & 148190 & 121090 & 45121 & 5218 & $119=0$ & $t$ & $\therefore \quad$ & $\therefore$ \\
\hline $740-\mathrm{G}$ & $80 \mathrm{~A}$ & 2.9 & 28777000 & 47873 & 131990 & 118840 & 38642 & 4963 & ç314 & ¿ & 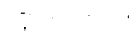 & $\cdots$ \\
\hline $740-G$ & 81 & 5.3 & 53237410 & 194550 & 207990 & 220350 & 104300 & 21770 & $29 \times 9 ?$ & $\therefore$ & $\because \quad:$ & $\theta^{\circ}$ \\
\hline $740-G$ & 82 & 3.3 & 33380000 & $<5$ & 98459 & 83013 & 42011 & 4629 & $145: 3$ & 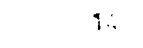 & & 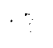 \\
\hline $740-G$ & 83 & 6.0 & 59712200 & 40504 & 58535 & 54697 & 22147 & 3572 & $905 j$ & 5 & & $\because \vdots$ \\
\hline $740-G$ & 84 & 0.0 & 29877 & 689 & 200 & 801 & $<5$ & 14 & 1905 & 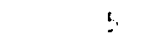 & & $\therefore$. \\
\hline $740-G$ & 85 & 0.0 & 190360 & 13280 & 2639 & 14599 & $<5$ & 1754 & 7473 & 3 & $\because \quad \vdots$ & bi \\
\hline $740-6$ & 86 & 1.1 & 10962000 & 115700 & 98743 & 84212 & 61176 & 15267 & 23349 & $\ddot{i}$ & $\therefore \ldots$ & $\varepsilon$ \\
\hline $740-6$ & 87 & 0.0 & 33395 & 2481 & 108 & 1784 & $<5$ & 56 & 1333 & 16 & $\because \quad \cdots$ & $\because \cdots$ \\
\hline $740-G$ & 88 & 0.0 & 497520 & 41540 & 35930 & 45052 & $<5$ & 2778 & 1658 & 3 & & $8 \vdots$ \\
\hline $740-G$ & 89 & 0.0 & 116730 & 7870 & 1879 & 4070 & 21 & 60 & $10 \leq 0 ?$ & $\therefore$ & $\because \because ?$ & $\varepsilon$ \\
\hline $740-G$ & 90 & 2.8 & 27625800 & 60874 & 93870 & 74844 & 83491 & 10085 & 22857 & $\ddot{i}$ & $\because \cdots$ & $6:$ \\
\hline $740-6$ & $90 \mathrm{~A}$ & 4.9 & 48920800 & 100950 & 155480 & 124870 & $1268<?$ & 16814 & $40: 24$ & 3 & $\because$. & ; \\
\hline $740-G$ & 91 & 0.0 & 3605 & 51 & 25 & $<5$ & $<5$ & 28 & $1: 0$ & 2 & $\therefore$ & c: \\
\hline $740-6$ & 92 & 0.0 & 325 & 20 & 8 & $<5$ & $<5$ & 16 & 8 & $\therefore$ & $i \ldots \ldots$ & 2 \\
\hline $740-6$ & 93 & 3.8 & 37714200 & 50471 & 116240 & 127150 & 58198 & 9884 & 24520 & & $\because \cdot \quad c$ & 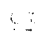 \\
\hline $740-G$ & 94 & 1.4 & 14140000 & 161320 & 72070 & 100440 & 3529 & 12012 & $276 \cdots$ & $:$ & 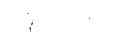 & $\therefore$ \\
\hline $740-G$ & 95 & 0.2 & 2306500 & 70855 & 39601 & 26836 & 18665 & 10465 & $10 \ldots$. & ; & ; & \\
\hline $740-G$ & 96 & 0.2 & 2472300 & 578 & 496 & 180 & 166 & 279 & $\therefore ?$ & - & & \\
\hline $740-G$ & 97 & 56.4 & 563750000 & 176420 & 297700 & 141170 & 202710 & 46632 & $72 . \because$ & . & & \\
\hline
\end{tabular}

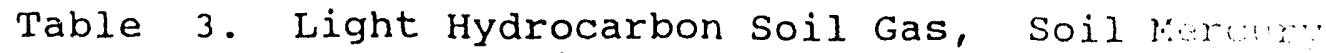




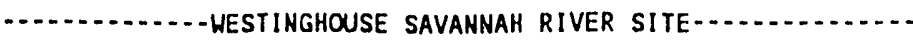

.... 740-G LANDFILL ....

SOIL GAS AND MERCURY CONCENTRATIONS a 3 FT.......

\begin{tabular}{|c|c|c|c|c|c|c|c|c|c|c|c|c|}
\hline $\begin{array}{c}\text { SAMPLE } \\
\#\end{array}$ & & $\begin{array}{r}\text { METHANE } \\
\mathbf{x}\end{array}$ & $\begin{array}{r}\text { METHANE } \\
\text { PPB }\end{array}$ & $\begin{array}{r}\text { ETHANE } \\
\text { PPB }\end{array}$ & $\begin{array}{r}\text { PROPANE } \\
\text { PPB }\end{array}$ & $\begin{array}{r}\text { I-BUTANE } \\
\text { PPB }\end{array}$ & $\begin{array}{c}N \text {-BUTANE } \\
\text { PPB }\end{array}$ & $\begin{array}{c}\text { ETHYLENE } \\
\text { PPE }\end{array}$ & $\begin{array}{c}\text { PROPYLENE } \\
\text { PPB }\end{array}$ & $\begin{array}{r}\text { MERCURY } \\
\mathrm{ng} / \mathrm{g}\end{array}$ & $\begin{array}{c}\text { SAMPLE } \\
\#\end{array}$ & \\
\hline $740-G$ & 98 & 0.0 & 353571 & 37545 & 17402 & 26639 & $<5$ & 463 & 10303 & 5 & $740-G$ & 98 \\
\hline $740-G$ & 99 & 0.0 & 225714 & 20972 & 2495 & 2576 & 62 & 400 & 11913 & 10 & $740-G$ & 99 \\
\hline $740-G$ & 100 & 25.1 & 251430000 & 156140 & 809680 & 682380 & 346990 & 77328 & 58729 & 2 & 740.6 & 100 \\
\hline $740-\mathrm{C}$ & $100 \mathrm{~A}$ & 18.7 & 187470000 & 183770 & 970070 & 966750 & 549270 & 86642 & 74174 & 3 & $740-6$ & $100 \mathrm{~A}$ \\
\hline $740-G$ & 101 & 0.0 & 13865 & 84 & 36 & 16 & 10 & 36 & 196 & 1 & $740-G$ & 101 \\
\hline $740-G$ & 102 & 0.0 & 44289 & 135 & 42 & $<5$ & $<5$ & 47 & 284 & 2 & $740-6$ & 102 \\
\hline $740-6$ & $103 \mathrm{~A}$ & 0.0 & 288571 & 687 & 497 & 624 & 206 & 329 & 1095 & 3 & $740-G$ & $103 \mathrm{~A}$ \\
\hline $740-G$ & 104 & 2.0 & 19710144 & 36490 & 39605 & 40463 & 14538 & 4513 & 14175 & 5 & $740-G$ & 104 \\
\hline $740-G$ & 105 & 0.0 & 767 & 6 & $<5$ & $<5$ & $<5$ & $<5$ & 5 & 7 & $740-6$ & 105 \\
\hline $740-G$ & 106 & 0.0 & 763 & 11 & $<5$ & $<5$ & $<5$ & 9 & $<5$ & $<1$ & $740-G$ & 106 \\
\hline $740-G$ & 107 & 8.6 & 85507200 & 26331 & 74221 & 59946 & 29525 & 4371 & 11260 & 3 & $740-6$ & 107 \\
\hline $740-G$ & 108 & 0.1 & 746376 & 7334 & 2169 & 2291 & $<5$ & 156 & 1546 & 2 & $740-G$ & 108 \\
\hline $740-G$ & 109 & 3.0 & 30434800 & 11512 & 27332 & 33645 & 4164 & 2062 & 9081 & 2 & $740-6$ & 109 \\
\hline $740-G$ & 110 & 58.7 & 586860000 & $<5$ & 10571 & 3478 & 3966 & 2569 & 3617 & 3 & $740-6$ & 110 \\
\hline $740-G$ & $110 \mathrm{~A}$ & 55.0 & 550320000 & $<5$ & 1591 & 366 & 775 & $<5$ & 1653 & 2 & $740-G$ & $110 \mathrm{~A}$ \\
\hline $740-G$ & 111 & 7.7 & 76812000 & 135500 & 181410 & 166540 & 123830 & 30271 & 45345 & 2 & $740-G$ & 111 \\
\hline $740-G$ & 112 & 0.0 & 61055 & 1035 & 1215 & 3770 & $<5$ & 37 & 4787 & 2 & $740-G$ & 112 \\
\hline $740-G$ & 113 & 0.7 & 6734200 & 4139 & 42667 & 51030 & 23935 & 1141 & 12373 & 5 & $740-G$ & 113 \\
\hline $740-6$ & 114 & 0.0 & 1355 & 94 & 77 & 233 & $<5$ & 30 & 1682 & 4 & $740-G$ & 114 \\
\hline $740-G$ & 115 & 17.3 & 172840000 & 93174 & 239320 & 202280 & 132150 & 24594 & 61746 & 5 & $740-G$ & 115 \\
\hline $740-G$ & 116 & 0.0 & 1424 & 157 & 45 & 10 & $<5$ & 83 & 3609 & 8 & $740-G$ & 116 \\
\hline $740-6$ & 117 & 9.5 & 95362318 & 32520 & 103790 & 80771 & 44763 & 10232 & 84962 & 5 & $740-G$ & 117 \\
\hline $740-G$ & 118 & 9.7 & 97101400 & 20407 & 59624 & 50552 & 25750 & 5985 & 32976 & 5 & $740-G$ & 118 \\
\hline $740-6$ & 119 & 0.0 & 727 & 10 & 6 & $<5$ & $<5$ & 7 & 11 & 2 & $740-G$ & 119 \\
\hline $740-6$ & 120 & 0.0 & 335 & 10 & $<5$ & $<5$ & $<5$ & 9 & 20 & $<1$ & $740-6$ & 120 \\
\hline $740-G$ & 121 & 2.6 & 26086956 & 1808 & 6557 & 21827 & 2434 & 1716 & 6762 & 2 & $740-G$ & 121 \\
\hline $740-6$ & 122 & 4.6 & 46376800 & 2180 & 10906 & 6433 & 2601 & 1530 & 3081 & 2 & $740-G$ & 122 \\
\hline $740-G$ & 123 & 0.6 & 5860000 & 4912 & 22739 & 20486 & 9394 & 1887 & 6678 & 5 & $740-G$ & 123 \\
\hline $740-G$ & 124 & 6.8 & 67647000 & 27850 & 96698 & 97239 & 57317 & 11910 & 22537 & 3 & $740-G$ & 124 \\
\hline $740-G$ & 125 & 48.6 & 485760000 & 25171 & 47308 & 49691 & 37218 & 17102 & 12987 & 3 & $740-6$ & 125 \\
\hline $740-6$ & 126 & 16.7 & 167058000 & 6816 & 45158 & 37824 & 21815 & 4239 & 15565 & 3 & $740-G$ & 126 \\
\hline $740-G$ & 127 & 23.3 & 232500000 & 4549 & 14514 & 14483 & 4418 & 1883 & 2753 & 17 & $740-G$ & 127 \\
\hline $740-G$ & 128 & 0.0 & 32600 & 106 & 34 & 10 & $<5$ & 64 & 2070 & 3 & $740-G$ & 128 \\
\hline $740-G$ & 129 & 6.6 & 66470600 & 6469 & 33187 & 37605 & 24009 & 2604 & 5684 & 3 & $740-6$ & 129 \\
\hline $740-6$ & $129 \mathrm{~A}$ & 14.5 & 145000000 & 12602 & 66672 & 74813 & 49644 & 4679 & 11224 & 3 & $740-6$ & $129 \mathrm{~A}$ \\
\hline $740-G$ & 130 & 13.5 & 135000000 & 6984 & 93306 & 55802 & 31442 & 4945 & 16373 & 3 & $740-G$ & 130 \\
\hline $740-G$ & 131 & 14.9 & 149490000 & 32132 & 81264 & 66297 & 41674 & 34087 & 20281 & 3 & $740-G$ & 131 \\
\hline $740-6$ & $131 \mathrm{~A}$ & 16.0 & 159705000 & 26534 & 65334 & 52308 & 32802 & 27847 & 18089 & 4 & $740-6$ & $131 \mathrm{~A}$ \\
\hline $740-G$ & 132 & 0.0 & 2959 & 103 & 38 & 22 & $<5$ & 131 & 2069 & 3 & $740-G$ & 132 \\
\hline $740-G$ & 133 & 0.0 & 2086 & 20 & 28 & 238 & $<5$ & 15 & 279 & $<1$ & $740-G$ & 133 \\
\hline $740-G$ & 134 & 0.0 & 5005 & 9 & 5 & $<5$ & $<5$ & 10 & 28 & $<1$ & $740-G$ & 134 \\
\hline $740-G$ & 135 & 0.9 & 8764400 & 269 & 611 & 409 & 386 & 155 & 412 & 2 & $740-6$ & 135 \\
\hline $740-G$ & 136 & 36.9 & 369130000 & $<5$ & 58803 & 36829 & 14631 & 3991 & 3694 & 1 & $740-G$ & 136 \\
\hline $740-G$ & 137 & 0.5 & 5231100 & 1978 & 4467 & 71519 & 3128 & 876 & 805 & 2 & $740-6$ & 137 \\
\hline $740-G$ & 138 & 34.3 & 343010000 & $<5$ & 3381 & 3149 & 434 & $<5$ & 680 & 2 & $740-6$ & 138 \\
\hline $740-G$ & 139 & 0.6 & 6354400 & 240 & 482 & 588 & 470 & 162 & 218 & 1 & $740-G$ & 139 \\
\hline $740-G$ & 140 & 27.8 & 277580000 & 10407 & 42803 & 49767 & 34329 & 5995 & 10232 & 2 & $740-G$ & 140 \\
\hline $740-G$ & $140 \mathrm{~A}$ & 24.3 & 243250000 & 9410 & 38056 & 44129 & 30515 & 5327 & 9164 & 1 & $740-G$ & $140 \mathrm{~A}$ \\
\hline $740-G$ & 141 & 0.0 & 332 & 21 & 7 & $<5$ & $<5$ & 10 & 11 & 3 & $740-G$ & 141 \\
\hline $740-G$ & 142 & 0.0 & 300 & 18 & 6 & $<5$ & $<5$ & 15 & 14 & 2 & $740-6$ & 142 \\
\hline $740-G$ & 143 & 0.0 & 202 & 11 & $<5$ & $<5$ & $<5$ & 10 & 22 & 3 & $740-G$ & 143 \\
\hline $740-G$ & 144 & 0.0 & 203 & 16 & 6 & $<5$ & $<5$ & 12 & $<5$ & 2 & $740-6$ & 144 \\
\hline $740-\mathrm{G}$ & 145 & 0.0 & 407 & 10 & $<5$ & $<5$ & $<5$ & 5 & $<5$ & 2 & $740-6$ & 145 \\
\hline
\end{tabular}

Table 3. Light Hydrocarbon Soil Gas, Soil Mercury Concentrations and System Blanks at the 740-G Sanitary Landfill 
WESTINGHOUSE SAVANNAH RIVER S!TE-..........................

740-G LANDFILL -...

SOIL GAS AND MERCURY CONCENTRATIONS a 3 FT......

SAMPLE METHANE METHANE ETHANE PROPANE I-BUTANE N-BUtANE ETHYLENE PROPYLENE mERCURY SAMPLE

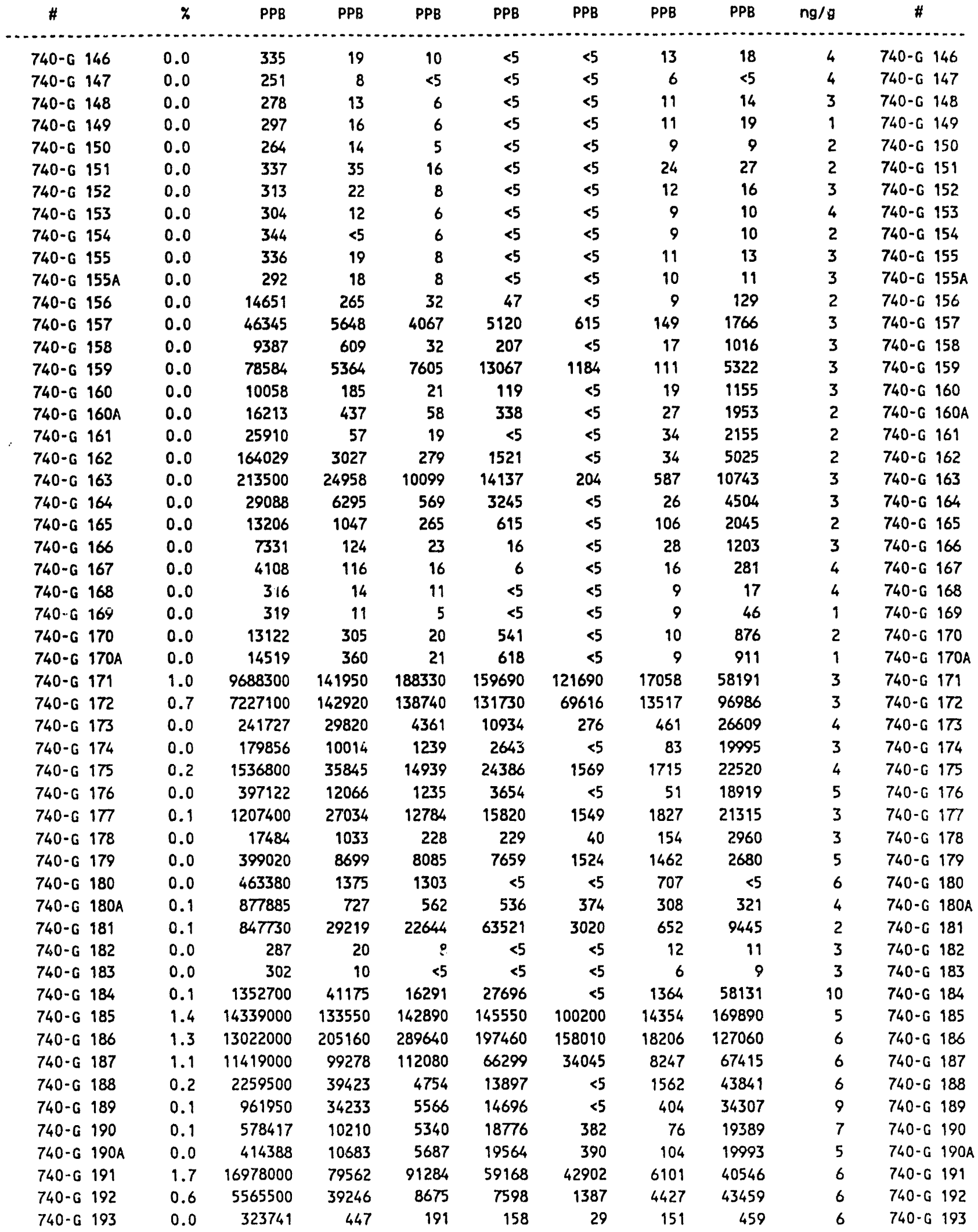

Table 3. Light Hydrocarbon Soil Gas, Soil Mercury Concentrations and System Blanks at the 740-G Sanitary Landfill 
-WESTINGHOUSE SAVANNAK RIVER SITE-...............

-... 740-G LANDFILL -...

SOIL GAS AND MERCURY CONCENTRATIONS \& $3 \mathrm{FT} \ldots . . .$.

\begin{tabular}{|c|c|c|c|c|c|c|c|c|c|c|}
\hline $\begin{array}{c}\text { SAMPLE } \\
\#\end{array}$ & $\begin{array}{r}\text { METHANE } \\
\mathbf{x}\end{array}$ & $\begin{array}{r}\text { METHANE } \\
\text { PPB }\end{array}$ & $\begin{array}{r}\text { ETHANE } \\
\text { PPB }\end{array}$ & $\begin{array}{r}\text { PROPANE } \\
\text { PPB }\end{array}$ & $\begin{array}{r}\text { I-BUTANE } \\
\text { PPB }\end{array}$ & $\begin{array}{c}\text { N-BUTANE E } \\
\text { PPB }\end{array}$ & $\begin{array}{r}\text { ETHYLENE } \\
\text { PPB }\end{array}$ & $\begin{array}{c}\text { PROPYLENE } \\
\text { PPB }\end{array}$ & $\begin{array}{r}\text { MERCURY } \\
n g / g\end{array}$ & $\begin{array}{c}\text { SAMPLE } \\
\#\end{array}$ \\
\hline $740-G \quad 194$ & 0.4 & 3712200 & 1202 & 1308 & 249 & 192 & 1195 & 295 & 6 & $740-\mathrm{G} 194$ \\
\hline $740-G \quad 195$ & 0.0 & 79137 & 3388 & 557 & 2421 & 15 & 23 & 5278 & 4 & $740-G 195$ \\
\hline $740-G 196$ & 0.0 & 597 & 19 & 8 & $<5$ & $<5$ & 13 & 75 & 1 & $740-G 196$ \\
\hline $740-G \quad 197$ & 0.0 & 520 & 14 & 6 & $<5$ & $<5$ & 10 & 9 & 1 & $740-G \quad 197$ \\
\hline $740-G 198$ & 0.0 & 3341 & 113 & 43 & 234 & $<5$ & 10 & 1189 & 3 & $740-G \quad 198$ \\
\hline $740-G 199$ & 1.9 & 19393939 & 216040 & 208440 & 188770 & 147110 & 16762 & 50934 & 5 & $740-G \quad 199$ \\
\hline $740-G 200$ & 5.5 & 55142800 & 71036 & 206030 & 106920 & 135120 & 10134 & 18245 & $<1$ & $740-G 200$ \\
\hline $740-\mathrm{G} 200 \mathrm{~A}$ & 5.2 & 52000000 & 70891 & 208910 & 114790 & 144900 & 9699 & 19661 & 3 & $740-G 200 A$ \\
\hline $740-G 201$ & 3.7 & 36571400 & 100780 & 178480 & 134080 & 177910 & 8250 & 105210 & 1 & $740-6201$ \\
\hline $740-G \quad 202$ & 0.7 & 6666666 & 44861 & 49565 & 19973 & 19011 & 3523 & 19447 & 5 & $740-G \quad 202$ \\
\hline $740-G \quad 203$ & 0.0 & 378571 & 432 & 69 & 145 & $<5$ & 29 & 5139 & 6 & $740-G 203$ \\
\hline $740-G \quad 204$ & 0.0 & 421290 & 2267 & 798 & 162 & $<5$ & 633 & 5849 & 4 & $740-6204$ \\
\hline $740-G \quad 205$ & 1.6 & 16000000 & 44681 & 76233 & 54322 & 43472 & 4648 & 14101 & 3 & $740-G 205$ \\
\hline $740-G 206$ & 6.0 & 59714200 & 63209 & 178960 & 103170 & 101750 & 8575 & 20556 & $<1$ & $740-6206$ \\
\hline $740-G \quad 207$ & 1.4 & 14251000 & 2688 & 4649 & 277 & 423 & 1735 & 1374 & 7 & $740-G \quad 207$ \\
\hline $740-G \quad 208$ & 13.6 & 136285600 & 85386 & 157250 & 91151 & 102740 & 17019 & 25266 & 2 & $740-6208$ \\
\hline $740-6209$ & 0.4 & 3883500 & 41311 & 20427 & 28126 & 3300 & 1510 & 27169 & 4 & $740-6209$ \\
\hline $740-G 210$ & 0.0 & 238 & 14 & $<5$ & $<5$ & $<5$ & 11 & 6 & 4 & $740-G 210$ \\
\hline $740-G \quad 210 A$ & 0.0 & 264 & 7 & $<5$ & $<5$ & $<5$ & 6 & $<5$ & 6 & $740-G \quad 210 \mathrm{~A}$ \\
\hline $740-G 211$ & 0.0 & 397 & 15 & 7 & $<5$ & $<5$ & 10 & 11 & 4 & $740-G 211$ \\
\hline $740-G \quad 212$ & 0.0 & 37584 & 4257 & 4510 & 8620 & 524 & 224 & 13017 & 2 & $740-6212$ \\
\hline $740-G \quad 213$ & 0.0 & 38225 & 1285 & 1234 & 947 & 1038 & 468 & 708 & 4 & $740-G \quad 213$ \\
\hline $740-G \quad 214$ & 0.6 & 6273100 & 169040 & 133510 & 158430 & 143590 & 12881 & 142760 & 3 & $740-G 214$ \\
\hline $740-G \quad 215$ & 0.2 & 2242600 & 25713 & 7814 & 8421 & 7493 & 2341 & 18177 & 3 & $740-6215$ \\
\hline $740-G 216$ & 0.0 & 75382 & 13325 & 14188 & 18803 & $<5$ & $<5$ & 16991 & 4 & $740-G 216$ \\
\hline $740-G \quad 217$ & 0.0 & 18782 & 37 & 313 & 114 & $<5$ & 397 & 415 & 2 & $740-G \quad 217$ \\
\hline $740-G 218$ & 0.0 & 82857 & 117 & 35 & 559 & $<5$ & 36 & 2440 & 3 & $740-G \quad 218$ \\
\hline $740-G 219$ & 0.0 & 33063 & 449 & 93 & 324 & $<5$ & 46 & 3004 & 9 & $740-6219$ \\
\hline $740-G 220$ & 26.1 & 260900000 & 220490 & 683020 & 130170 & 169070 & 9002 & 6595 & 4 & $740-6220$ \\
\hline $740-G 220 A$ & 20.8 & 207670000 & 125040 & 284660 & 52648 & 64044 & 7037 & 4935 & 4 & $740-G 220 A$ \\
\hline $740-G \quad 221$ & 5.1 & 50571400 & 53345 & 104820 & 45813 & 46493 & 7261 & 27387 & 6 & $740-G 221$ \\
\hline $740-G 222$ & 1.2 & 11589000 & 10787 & 9186 & 1644 & 1564 & 7942 & 12090 & 5 & $740-G 222$ \\
\hline $740-G 223$ & 0.0 & 774 & 24 & 12 & $<5$ & $<5$ & 18 & 137 & $<1$ & $740-G 223$ \\
\hline $740-G 224$ & 0.0 & 357 & 19 & 11 & $<5$ & $<5$ & 15 & 22 & $<1$ & $740-G 224$ \\
\hline $740-G 225$ & 0.0 & 367 & 13 & 6 & $<5$ & $<5$ & 13 & 16 & 2 & $740-G 225$ \\
\hline $740-G 226$ & 0.0 & 1170 & 22 & 10 & $<5$ & $<5$ & 20 & 285 & 2 & $740-6226$ \\
\hline $740-G \quad 227$ & 0.0 & 93563 & 2272 & 967 & 1800 & 132 & 185 & 5535 & 4 & $740-G \quad 227$ \\
\hline $740-G 228$ & 0.0 & 13792 & 867 & 127 & 286 & 76 & 69 & 1490 & 4 & $740-G \quad 228$ \\
\hline $740-G 229$ & 0.0 & 36569 & 1012 & 1792 & 944 & 2038 & 526 & 600 & 3 & $740-0229$ \\
\hline $740-G 230$ & 0.0 & 7705 & 1833 & 134 & 55 & 60 & 43 & 825 & 5 & $740-6 \quad 230$ \\
\hline $740-G \quad 230 A$ & 0.0 & 8067 & 2015 & 148 & 61 & 31 & 28 & 1365 & 5 & $740-G 230 \mathrm{~A}$ \\
\hline $740-G 231$ & 0.0 & 6579 & 31 & 13 & $<5$ & 27 & 21 & 24 & 4 & $740-6231$ \\
\hline $740-G 232$ & 0.0 & 322222 & 4664 & 5614 & 3347 & 2200 & 128 & 367 & 2 & $740-G 232$ \\
\hline $740-G \quad 233$ & 0.0 & 45043 & 792 & 218 & 222 & 67 & 75 & 467 & 4 & $740-G 233$ \\
\hline $740-G 234$ & 0.0 & 6756 & 127 & 48 & 6 & 74 & 68 & 76 & 9 & $740-G 234$ \\
\hline $740-G 235$ & 0.0 & 1194 & 60 & 20 & $<5$ & 26 & 45 & 62 & 4 & $740-G 235$ \\
\hline $740-G 236$ & 0.3 & 3189000 & 955 & 2348 & 388 & 541 & 405 & 475 & 4 & $740-G 236$ \\
\hline $740-G \quad 237$ & 0.0 & 41635 & 2227 & 1503 & 2586 & 240 & 12 & 1134 & 3 & $740-G \quad 237$ \\
\hline $740-G 238$ & 0.0 & 362 & 13 & 5 & $<5$ & $<5$ & 12 & 8 & 1 & $740-G \quad 238$ \\
\hline $740-G 239$ & 0.0 & 413 & 23 & 9 & $<5$ & 15 & 18 & 19 & 4 & $740-G \quad 239$ \\
\hline $740-G 240$ & 0.0 & 560 & 35 & 11 & $<5$ & 16 & 23 & 18 & 4 & $740-G 240$ \\
\hline $740-\mathrm{G} 240 \mathrm{~A}$ & 0.0 & 608 & 32 & 12 & $<5$ & 13 & 21 & 19 & 2 & $740-G 240 \mathrm{~A}$ \\
\hline $740-G 241$ & 0.0 & 406 & 24 & 9 & $<5$ & 13 & 16 & 26 & 2 & $740-G 241$ \\
\hline
\end{tabular}

Table 3. Light Hydrocarbon Soil Gas, Soil Mercury Concentrations and System Blanks at the 740-G Sanitary Landfill 


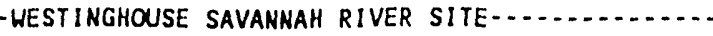

.... 740-G LANDFILL -...

SOIL GAS AND MERCURY CONCENTRATIONS D 3 FT......

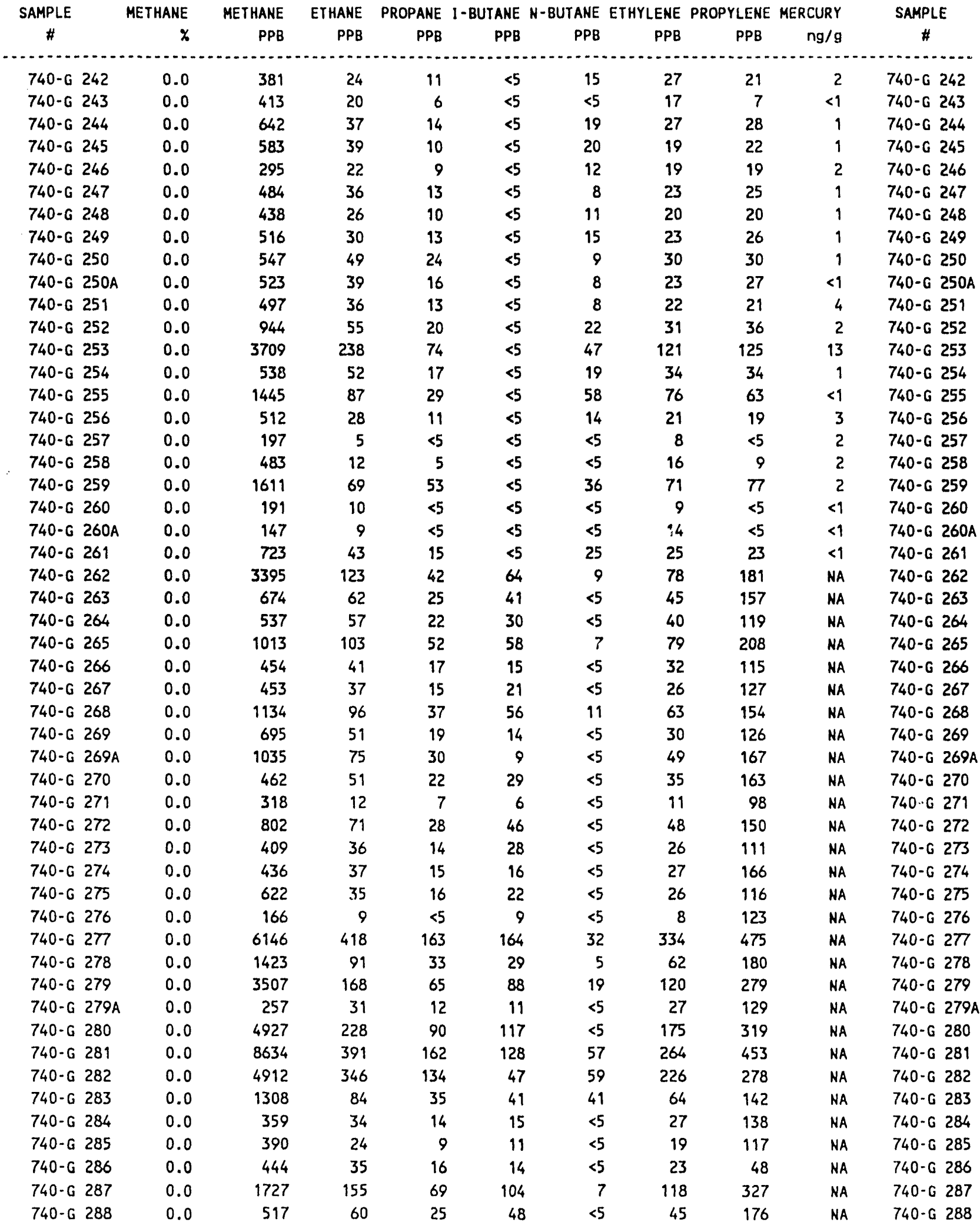

Table 3. Light Hydrocarbon Soil Gas, Soil Mercury Concentrations and system Blanks at the 740-G Sanitary Landfill 


\begin{tabular}{|c|c|c|c|c|c|c|c|c|c|c|}
\hline $\begin{array}{c}\text { SAMPLE } \\
\#\end{array}$ & $\begin{array}{r}\text { METHANE } \\
\%\end{array}$ & $\begin{array}{r}\text { METHANE } \\
\text { PPB }\end{array}$ & $\begin{array}{r}\text { ETHANE } \\
\text { PPB }\end{array}$ & $\begin{array}{c}\text { PROPANE } \\
\text { PPB }\end{array}$ & $\begin{array}{r}\text { I-BUTANE } \\
\text { PPB }\end{array}$ & $\begin{array}{r}\text { N-BUTANE } \\
\text { PPB }\end{array}$ & $\begin{array}{r}\text { ETHYLENE } \\
\text { PPB }\end{array}$ & $\begin{array}{c}\text { PROPYLENE } \\
\text { PPB }\end{array}$ & $\begin{array}{r}\text { MERCURY } \\
\mathrm{ng} / \mathrm{g}\end{array}$ & $\begin{array}{c}\text { SAMPLE } \\
\#\end{array}$ \\
\hline $740-G$ SB 1 & 0.0 & 2584 & 7 & $<5$ & $<5$ & $<5$ & $<5$ & $<5$ & NA & $740-G$ SB1 \\
\hline $740-G$ SB2 & 0.0 & 8935 & $<5$ & $<5$ & $<5$ & $<5$ & $<5$ & $<5$ & NA & $740-G$ SB2 \\
\hline $740-G$ SB3 & 0.0 & 14145 & $<5$ & $<5$ & $<5$ & $<5$ & $<5$ & $<5$ & NA & $740-G S B 3$ \\
\hline $740-6$ SB4 & 0.0 & 6397 & 5 & $<5$ & $<5$ & $<5$ & $<5$ & $<5$ & NA & $740-G$ SB4 \\
\hline $740-6$ SB5 & 0.0 & 6198 & $<5$ & $<5$ & $<5$ & $<5$ & $<5$ & $<5$ & NA & $740-G$ SB5 \\
\hline $740-6$ SB6 & 0.0 & 3292 & $<5$ & $<5$ & $<5$ & $<5$ & $<5$ & $<5$ & NA & $740-6$ SB 6 \\
\hline $740-6$ SB7 & 0.0 & 5589 & 8 & $<5$ & $<5$ & $<5$ & $<5$ & $<5$ & NA & $740-6587$ \\
\hline $740-G$ SB8 & 0.0 & 4687 & 6 & $<5$ & $<5$ & $<5$ & $<5$ & $<5$ & NA & $740-6$ SB8 \\
\hline $740-G$ SB9 & 0.0 & 2552 & 7 & $<5$ & $<5$ & $<5$ & $<5$ & $<5$ & NA & $740-6$ SB9 \\
\hline $740-G$ SB 10 & 0.0 & 9820 & 8 & 13 & 8 & 5 & $<5$ & $<5$ & NA & $740-G$ SB10 \\
\hline $740-G 5811$ & 0.0 & 2871 & 7 & 6 & $<5$ & $<5$ & $<5$ & $<5$ & NA & $740-G$ SB 11 \\
\hline $740-G$ SB12 & 0.0 & 3486 & 8 & 13 & $<5$ & $<5$ & 5 & $<5$ & NA & $740-G$ SB12 \\
\hline $740-\mathrm{G} S \mathrm{SB} 13$ & 0.0 & 5528 & 6 & $<5$ & $<5$ & $<5$ & $<5$ & $<5$ & NA & $740-G$ SB13 \\
\hline $740-G$ SB14 & 0.0 & 2848 & $<5$ & $<5$ & $<5$ & $<5$ & $<5$ & $<5$ & NA & $740-6$ SB14 \\
\hline $740-G$ SB 15 & 0.0 & 2527 & 6 & 5 & $<5$ & $<5$ & $<5$ & $<5$ & HA & $740-G$ SB15 \\
\hline $740-G$ SB16 & 0.0 & 4921 & 5 & 7 & $<5$ & $<5$ & 7 & $<5$ & NA & $740-G$ SB 16 \\
\hline $740-\mathrm{G}$ SB 17 & 0.0 & 2562 & 6 & $<5$ & $<5$ & $<5$ & 7 & $<5$ & NA & $740-6$ SB17 \\
\hline $740-G$ SB 18 & 0.0 & 2586 & 6 & $<5$ & $<5$ & $<5$ & $<5$ & $<5$ & NA & $740-G$ SB 18 \\
\hline $740-G$ SB19 & 0.0 & 2611 & 5 & $<5$ & $<5$ & $<5$ & $<5$ & $<5$ & NA & $740-G$ SB 19 \\
\hline $740-G$ SB2O & 0.0 & 2546 & 5 & $<5$ & $<5$ & $<5$ & 7 & $<5$ & NA & $740-G$ SB20 \\
\hline $740-G$ SB21 & 0.0 & 2619 & 5 & $<5$ & $<5$ & $<5$ & $<5$ & $<5$ & NA & $740-6$ SB21 \\
\hline $740-G$ SB22 & 0.0 & 2541 & $<5$ & $<5$ & $<5$ & $<5$ & $<5$ & $<5$ & NA & $740-G$ SB22 \\
\hline $740-G$ SB23 & 0.0 & 3010 & $<5$ & 5 & $<5$ & $<5$ & $<5$ & $<5$ & NA & $740-G$ SB23 \\
\hline $740-6$ SB24 & 0.0 & 2489 & 9 & $<5$ & $<5$ & $<5$ & $<5$ & $<5$ & NA & $740-G$ SB24 \\
\hline $740-G$ SB25 & 0.0 & 3789 & $<5$ & 6 & $<5$ & $<5$ & $<5$ & $<5$ & NA & $740-G$ SB25 \\
\hline $740-G \quad S B 26$ & 0.0 & 1542 & 5 & $<5$ & $<5$ & $<5$ & 10 & 8 & NA & $740-G$ SB26 \\
\hline $740-G \quad S B 27$ & 0.0 & 1554 & $<5$ & $<5$ & $<5$ & $<5$ & 6 & $<5$ & NA & $740-G \quad S B 27$ \\
\hline $740-G$ SB28 & 0.0 & 1549 & $<5$ & $<5$ & $<5$ & $<5$ & 6 & $<5$ & NA & $740-G$ SB28 \\
\hline $740-G$ SB29 & 0.0 & 1545 & $<5$ & $<5$ & $<5$ & $<5$ & 8 & $<5$ & NA & $740-G$ SB29 \\
\hline $740-G \quad S B 30$ & 0.0 & 1578 & $<5$ & $<5$ & $<5$ & $<5$ & 7 & $<5$ & NA & $740-G$ SB30 \\
\hline $740-G$ SB31 & 0.0 & 1572 & $<5$ & $<5$ & $<5$ & $<5$ & 9 & $<5$ & NA & $740-G \quad S B 31$ \\
\hline $740-G \quad S B 32$ & 0.0 & 1661 & $<5$ & 89 & $<5$ & $<5$ & $<5$ & $<5$ & NA & $740-G_{1} S B 32$ \\
\hline $740-G S 833$ & 0.0 & 1680 & $<5$ & $<5$ & $<5$ & $<5$ & $<5$ & 105 & NA & $740-6 \quad S B 33$ \\
\hline $740-G \quad S B 34$ & 0.0 & 1735 & 5 & $<5$ & $<5$ & $<5$ & $<5$ & 101 & NA & $740-G$ SB34 \\
\hline
\end{tabular}

Table 3. Light Hydrocarbon Soil Gas, Soil Mercury Concentrations and System Blanks at the 740-G Sanitary Landfill 
This page intentionally left blank. 


\begin{tabular}{rrrrrrr} 
COMPOUND & RANGE & RANGE & RANGE & RANGE & RANGE & RANGE \\
& 1 & 2 & 3 & 4 & 5 & 6 \\
\hline PENTANE & 942.19 & 91.52 & 9.13 & 0.91 & 0.091 & 0.0091 \\
11 DICHLOROETHYLENE & 1358.80 & 131.99 & 13.16 & 1.32 & 0.132 & 0.0132 \\
METHYLENE CHLORIDE & 1694.16 & 164.57 & 16.41 & 1.64 & 0.164 & 0.0164 \\
TRANS 1,2 DICRLORO & 1408.09 & 136.78 & 13.64 & 1.36 & 0.136 & 0.0136 \\
11 DICHOROETHANE & 1291.56 & 125.46 & 12.51 & 1.25 & 0.125 & 0.0125 \\
CHIOROFORM & 1356.81 & 131.80 & 13.14 & 1.31 & 0.131 & 0.0131 \\
111 TRICHLOROETHANE & 1089.10 & 105.79 & 10.55 & 1.05 & 0.105 & 0.0105 \\
CARBON TETRACHLORIDE & 1125.32 & 109.31 & 10.90 & 1.09 & 0.109 & 0.0109 \\
HEPTANE & 741.22 & 72.00 & 7.18 & 0.72 & 0.072 & 0.0072 \\
BENZENE & 1214.92 & 118.02 & 11.77 & 1.18 & 0.118 & 0.0118 \\
TRICHLOROETHYLENE & 1208.33 & 117.38 & 11.70 & 1.17 & 0.117 & 0.0117 \\
OCIANE & 668.30 & 64.92 & 6.47 & 0.65 & 0.065 & 0.0065 \\
TOLUENE & 1021.92 & 99.27 & 9.90 & 0.99 & 0.099 & 0.0099 \\
TETRACHLOROETHYLENE & 1062.81 & 103.24 & 10.29 & 1.03 & 0.103 & 0.0103 \\
NONANE & 607.90 & 59.05 & 5.89 & 0.59 & 0.059 & 0.0059 \\
ETHYL BENZENE & 886.78 & 86.14 & 8.59 & 0.86 & 0.086 & 0.0086 \\
M-XYLENE & 883.71 & 85.84 & 8.56 & 0.86 & 0.086 & 0.0086 \\
P-XYLENE & 880.64 & 85.54 & 8.53 & 0.85 & 0.085 & 0.0085 \\
O-XYLENE & 888.83 & 86.34 & 8.61 & 0.86 & 0.086 & 0.0086 \\
DECANE & 557.12 & 54.12 & 5.40 & 0.54 & 0.054 & 0.0054
\end{tabular}

Table 4. VOC Prepared Standard Concentrations 
This page intentionally left blank. 
STAMDARD GC $\quad$ ETHYL M\&Pname area cycle pentane hexane heptane benzene octane toluene honane benzene Xylene xylenf. decane

\begin{tabular}{|c|c|c|c|c|c|c|c|c|c|c|c|c|c|}
\hline STO I L6 & $740-G$ & W2 4 & NA & NA & NA & NA & NA & NA & $\mathrm{NA}$ & $N A$ & NA & NA & NA \\
\hline STD 1 L6 & $740-G$ & H2 5 & NA & NA & NA & NA & NA & NA & NA & NA & $N /$ & NA & NA \\
\hline STO $\perp 15$ & $740-G$ & W2 6 & NA & NA & NA & NA & NA & NA & NA & NA & NA & NA & NA \\
\hline STO $\mid$ L5 & $740-G$ & H2 7 & NA & NA & NA & NA & NA & NA & NA & NA & NA & HA & NA \\
\hline $\begin{array}{l}\text { STO I L5 } \\
\text { MEAN }\end{array}$ & $740-6$ & W2 263 & MA & HA & NA & MA & NA & NA & NA & NA & NA & NA & NA \\
\hline
\end{tabular}

\begin{tabular}{|c|c|c|c|c|c|c|c|c|c|c|c|c|c|}
\hline STD \& L4 & $740-G$ & W2 8 & NA & NA & 0.73 & 1.19 & 0.67 & 1.04 & 0.62 & 0.89 & 1.78 & 0.87 & 0.55 \\
\hline STD $1<4$ & $740-G$ & W2 9 & NA & NA & 0.72 & 1.18 & 0.64 & 1.00 & 0.61 & 0.88 & 1.75 & 0.86 & 0.55 \\
\hline STO I L4 & $740-G$ & 4225 & HA & NA & 0.72 & 1.18 & 0.67 & 0.93 & 0.60 & 0.87 & 1.68 & 0.82 & 0.55 \\
\hline STO \& L4 & $740-G$ & W2 37 & MA & NA & 0.71 & 1.15 & 0.63 & 0.95 & 0.58 & 0.84 & 1.66 & 0.84 & 0.53 \\
\hline STO \& L4 & $740-6$ & H2 49 & HA & NA & 0.74 & 1.23 & 0.66 & 0.91 & 0.62 & 0.90 & 1.77 & 0.97 & 0.58 \\
\hline STD I L4 & $740-G$ & W2 61 & MA & NA & 0.72 & 1.18 & 0.68 & 0.84 & 0.61 & 0.87 & 1.71 & 0.87 & 0.55 \\
\hline STO I L4 & $740-G$ & W2 73 & MA & NA & 0.73 & 1.19 & 0.64 & 1.04 & 0.58 & 0.86 & 1.70 & 0.87 & 0.54 \\
\hline STO 1 L4 & $740-G$ & W2 86 & NA & NA & 0.69 & 1.13 & 0.64 & 0.99 & 0.55 & 0.82 & 1.60 & 0.86 & 0.53 \\
\hline STO I L4 & $740-G$ & W2 97 & NA & NA & 0.68 & 1.16 & 0.65 & 0.93 & 0.58 & 0.85 & 1.70 & 0.85 & 0.53 \\
\hline STD $\&$ L4 & $740-G$ & W2 110 & NA & NA & 0.68 & 1.13 & 0.61 & 0.93 & 0.55 & 0.81 & 1.60 & 0.81 & 0.52 \\
\hline STO I L4 & $740-G$ & W2 121 & NA & HA & 0.73 & 1.20 & 0.65 & 0.94 & 0.60 & 0.89 & 1.80 & 0.88 & 0.55 \\
\hline STD \& L4 & $740-G$ & W2 133 & NA & NA & 0.71 & 1.17 & 0.65 & 0.98 & 0.58 & 0.87 & 1.73 & 0.86 & 0.53 \\
\hline STD \& L4 & $740-G$ & W2 146 & NA & NA & 0.71 & 1.18 & 0.67 & 1.02 & 0.60 & 0.88 & 1.74 & 0.88 & 0.52 \\
\hline STD I L4 & $740-G$ & W2 158 & NA & NA & 0.71 & 1.16 & 0.64 & 0.93 & 0.57 & 0.86 & 1.72 & 0.89 & 0.52 \\
\hline STD I 14 & $740-G$ & W2 171 & NA & NA & 0.72 & 1.19 & 0.66 & 1.01 & 0.59 & 0.88 & 1.74 & 0.89 & 0.54 \\
\hline STD $1 \mathrm{~L} 4$ & $740-G$ & W2 189 & NA & NA & 0.73 & 1.19 & 0.68 & 0.97 & 0.57 & 0.87 & 1.74 & 0.89 & 0.54 \\
\hline STO I L4 & $740-6$ & W2 201 & NA & NA & 0.73 & 1.20 & 0.69 & 1.07 & 0.63 & 0.88 & 1.74 & 0.89 & 0.79 \\
\hline STD $1 \mathrm{~L} 4$ & $740-G$ & W2 213 & NA & NA & 0.67 & 1.06 & 0.64 & 0.97 & 0.56 & 0.86 & 1.74 & 0.82 & 0.50 \\
\hline STD \& L4 & $740-G$ & W2 237 & NA & NA & 0.72 & 1.19 & 0.65 & 0.99 & 0.58 & 0.87 & 1.74 & 0.86 & 0.52 \\
\hline STD I L4 & $740-G$ & WR 286 & MA & NA & 0.71 & 1.17 & 0.65 & 0.97 & 0.57 & 0.86 & 1.72 & 0.88 & 0.53 \\
\hline STO I L4 & $740-G$ & W2 298 & NA & NA & 0.70 & 1.15 & 0.63 & 0.94 & 0.56 & 0.83 & 1.65 & 0.88 & 0.52 \\
\hline MEAN & & & & & 0.71 & 1.17 & 0.65 & 0.97 & 0.58 & 0.86 & 1.71 & 0.87 & 0.55 \\
\hline STD DEV & & & & & 0.02 & 0.03 & 0.02 & 0.05 & 0.02 & 0.02 & 0.05 & 0.03 & 0.06 \\
\hline \% STD DEV & & & & & 2.45 & 2.89 & 2.96 & 5.37 & 3.89 & 2.61 & 3.09 & 3.65 & 10.45 \\
\hline STD I L3 & $740-G$ & H2 10 & 8.06 & NA & 6.89 & 11.34 & 6.25 & 9.51 & 5.69 & 8.30 & 16.55 & 8.38 & 5.25 \\
\hline STD $\mid$ L3 & $740-G$ & W2 11 & 8.16 & NA & 6.90 & 11.33 & 6.25 & 9.50 & 5.71 & 8.30 & 16.51 & 8.29 & 5.23 \\
\hline STD I L3 & $740-G$ & W2 177 & 8.15 & NA & 6.91 & 11.37 & 6.30 & 9.54 & 5.71 & 8.35 & 16.56 & 8.39 & 5.19 \\
\hline STD 1 L3 & $740-G$ & H2 225 & 8.11 & NA & 6.90 & 11.36 & 6.31 & 9.66 & 5.72 & 8.38 & 16.69 & 8.44 & 5.21 \\
\hline STD $1 \mathrm{L3}$ & $740-G$ & W2 249 & 7.95 & NA & 6.82 & 11.22 & 6.22 & 9.51 & 5.65 & 8.27 & 16.47 & 8.35 & 5.18 \\
\hline STD $\perp$ L3 & $740-G$ & W2 310 & $7 . \pi$ & NA & 6.71 & 11.05 & 6.09 & 9.33 & 5.54 & 8.06 & 16.06 & 8.12 & 5.01 \\
\hline MEAN & & & 8.03 & & 6.86 & 11.28 & 6.24 & 9.51 & 5.67 & 8.28 & 16.47 & 8.33 & 5.18 \\
\hline STD DEV & & & 0.14 & & 0.07 & 0.11 & 0.07 & 0.90 & 0.06 & 0.10 & 0.20 & 0.10 & 0.08 \\
\hline * STO DEV & & & 1.74 & & 1.06 & 1.01 & 1.15 & 1.02 & 1.13 & 1.24 & 1.19 & 1.24 & 1.55 \\
\hline STO 1 L2 & $740-G$ & W2 12 & 107.70 & NA & 74.95 & 122.90 & 68.03 & 103.92 & 62.09 & 90.48 & 180.30 & 90.84 & 57.08 \\
\hline STD 1 L2 & $740-G$ & W2 13 & 108.72 & NA & 75.82 & 124.42 & 68.65 & 104.95 & 62.52 & 91.24 & 181.52 & 91.53 & 57.30 \\
\hline STO 224 & $740-6$ & W2 15 & 1003.62 & 1035.00 & NA & NA & NA & NA & NA & NA & NA & NA & NA \\
\hline STD 224 & $740-G$ & W2 74 & 1023.28 & 1061.15 & NA & NA & NA & NA & NA & NA & NA & NA & NA \\
\hline STO 224 & $740-G$ & W2 176 & 1022.20 & 1058.75 & NA & NA & NA & NA & NA & NA & NA & NA & NA \\
\hline STD VC 1000 & $740-6$ & W2 16 & NA & NA & NA & NA & NA & NA & NA & NA & NA & NA & NA \\
\hline STD VC 1000 & $740-G$ & W2 175 & NA & NA & NA & NA & NA & NA & NA & NA & NA & NA & NA \\
\hline
\end{tabular}




\begin{tabular}{|c|c|c|c|c|c|c|c|c|c|c|}
\hline \multirow[b]{2}{*}{ STANDARD } & \multirow[b]{3}{*}{ AREA } & \multirow[b]{2}{*}{ GC } & \multirow{3}{*}{$\begin{array}{r}\text { VINYL } \\
\text { CHLORIDE }\end{array}$} & \multirow{3}{*}{$\begin{array}{r}\text { METHYLLENE } \\
\text { CHLORIDE }\end{array}$} & \multicolumn{2}{|l|}{ TRANS 1,2} & \multirow{2}{*}{$\begin{array}{l}111 \text { TRI } \\
\text { CHLORO }\end{array}$} & \multirow{2}{*}{$\begin{array}{r}\text { CARBOH } \\
\text { TETRA }\end{array}$} & \multirow{3}{*}{$\begin{array}{l}\text { TRICLORO } \\
\text { ETHYLENE }\end{array}$} & \multirow{3}{*}{$\begin{array}{r}\text { TETRA } \\
\text { CHLCRO } \\
\text { ETHYLENE }\end{array}$} \\
\hline & & & & & DICHLORO & CHLORO & & & & \\
\hline NAME & & CYCLE & & & ETHYLENE & FORM & ETHANE & CHLORIDE & & \\
\hline STD I L6 & $740-6$ & W2 4 & NA & NA & NA & 0.013 & 0.010 & 0.011 & 0.012 & 0.010 \\
\hline STD I L6 & $740-6$ & W2 5 & HA & NA & HA & 0.013 & 0.011 & 0.011 & 0.012 & $0 .(16$ \\
\hline STD I LS & $740-G$ & W2 6 & HA & NA & 0.15 & 0.131 & 0.104 & 0.109 & 0.119 & c.10: \\
\hline STD $1 \angle 5$ & $740-G$ & W2 7 & NA & NA & 0.15 & 0.130 & 0.104 & 0.108 & 0.117 & 0.10 \\
\hline STD I LS & $740-G$ & W2 263 & NA & HA & 0.16 & 0.188 & 0.148 & 0.112 & 0.162 & 0.103 \\
\hline MEAN & & & & & 0.15 & 0.150 & 0.119 & 0.110 & 0.133 & $0.10 j$ \\
\hline STD DEV & & & & & 0.00 & 0.023 & 0.018 & 0.001 & 0.018 & 0.000 \\
\hline \% STD DEV & & & & & 1.78 & 15.686 & 15.137 & 1.342 & 13.550 & 0.375 \\
\hline STD \ L4 & $740-6$ & W2 8 & NA & 1.65 & 1.28 & 1.352 & 1.059 & 1.117 & 1.203 & 1.17 \\
\hline STD 1 L4 & $740-G$ & W2 9 & NA & 1.64 & 1.27 & 1.340 & 1.054 & 1.066 & 1.193 & $1.0 \therefore$ \\
\hline STD \& L4 & $740-G$ & H2 25 & NA & 1.71 & 1.31 & 1.392 & 1.060 & 1.068 & 1.212 & $1.0 \%$ \\
\hline STO I L4 & $740 \cdot G$ & W2 37 & MA & 1.62 & 1.25 & 1.291 & 1.033 & 1.045 & 1.125 & $0.9,3$ \\
\hline STD I 1.4 & $740-G$ & H2 49 & NA & 1.70 & 1.35 & 1.555 & 1.160 & 1.074 & 1.328 & $1.1 \%$ \\
\hline STD I L4 & $740-G$ & W2 61 & NA & 1.67 & 1.27 & 1.344 & 1.065 & 1.112 & 1.159 & 1.62 \\
\hline STD \& L4 & $740-6$ & 1273 & NA & 1.66 & 1.34 & 1.496 & 1.147 & 1.216 & 1.281 & $1.12 \%$ \\
\hline STO : L4 & $740-G$ & W2 86 & NA & 1.82 & 1.27 & 1.355 & 1.104 & 1.075 & 1.173 & 1.027 \\
\hline STD $1 \angle 4$ & $740-G$ & W2 97 & NA & 1.64 & 1.37 & 1.554 & 1.180 & 1.194 & 1.326 & 1.156 \\
\hline STD I L4 & $740-6$ & H2 110 & NA & 1.50 & 1.31 & 1.440 & 1.120 & 1.086 & 1.215 & 1.044 \\
\hline STD $1 \mathrm{L4}$ & $740-G$ & W2 121 & NA & 1.54 & 1.40 & 1.191 & 1.275 & 1.140 & 1.410 & 1.202 \\
\hline STD $\&$ L4 & $740-G$ & W2 133 & NA & 1.60 & 1.32 & 1.599 & 1.185 & 1.139 & 1.246 & 1.076 \\
\hline STD I L4 & $740-G$ & W2 146 & MA & 1.60 & 1.33 & 1.637 & 1.202 & 1.209 & 1.263 & $1.14 i$ \\
\hline STD I L4 & $740-G$ & H2 158 & NA & 1.66 & 1.32 & 1.582 & 1.186 & 1.146 & 1.245 & 1.055 \\
\hline STD 1 L4 & $740-6$ & W2 171 & NA & 1.59 & 1.35 & 1.271 & 1.231 & 1.203 & 1.291 & 1.105 \\
\hline STD $\ \angle 4$ & $740-G$ & W2 189 & NA & 1.60 & 1.32 & 1.690 & 1.224 & 1.169 & 1.255 & $1 . C=$ \\
\hline STD I L4 & $740-G$ & W2 201 & NA & 1.80 & 1.32 & 1.271 & 1.220 & 1.122 & 1.287 & $1.1 \mathrm{in}$ \\
\hline STD I L4 & $740-6$ & H2 213 & NA & 2.57 & 1.22 & 1.361 & 1.080 & 1.111 & 1.096 & $0.52 i$ \\
\hline STD I L4 & $740-G$ & H2 237 & NA & 1.62 & 1.35 & 1.356 & 1.076 & 1.131 & 1.183 & 1.106 \\
\hline STD \& L4 & $740-G$ & W2 286 & NA & 1.61 & 1.36 & 1.372 & 1.082 & 1.144 & 1.191 & 1.110 \\
\hline STO 114 & $740-G$ & H2 298 & NA & 1.78 & 1.30 & 1.351 & 1.074 & 1.039 & 1.154 & $1.03 \%$ \\
\hline MEAN & & & & 1.69 & 1.31 & 1.419 & 1.134 & 1.124 & 1.230 & $1.6 \%$ \\
\hline STD DEV & & & & 0.21 & 0.04 & 0.133 & 0.070 & 0.052 & 0.073 & $0.0 \ldots$ \\
\hline * STD DEV & & & & 12.47 & 3.19 & 9.36 & 6.20 & 4.63 & 5.95 & C.E \\
\hline STD $1 \angle 3$ & $740-6$ & W2 10 & HA & 15.70 & 11.20 & 12.621 & 10.131 & 10.891 & 11.178 & $9.45:$ \\
\hline STD $1 \angle 3$ & $740-G$ & W2 11 & NA & 15.75 & 11.08 & 12.645 & 10.139 & 10.907 & 11.206 & 8.991 \\
\hline STD 1 L3 & $740-G$ & W2 177 & NA & 15.57 & 11.99 & 12.377 & 10.164 & 11.431 & 11.354 & $9.55 i$ \\
\hline STO 1 L3 & $740-G$ & W2 225 & NA & 15.41 & 11.88 & 12.301 & 10.126 & 11.604 & 11.199 & $9.05 \%$ \\
\hline STD 1 L3 & $740-G$ & W2 249 & NA & 15.26 & 11.67 & 12.761 & 10.288 & 11.458 & 11.430 & 9.065 \\
\hline STD I 13 & $740-6$ & W2 310 & NA & 14.91 & 11.69 & 12.687 & 10.166 & 10.687 & 11.260 & 9.123 \\
\hline MEAN & & & & 15.43 & 11.58 & 12.565 & 10.169 & 11.163 & 11.271 & 9.35 .5 \\
\hline STD DEV & & & & 0.28 & 0.34 & 0.167 & 0.055 & 0.346 & 0.092 & $0.33:$ \\
\hline * STD DEV & & & & 1.85 & 2.92 & 1.33 & 0.54 & 3.10 & 0.81 & 3.5 \\
\hline STO \& L2 & $740-G$ & H2 12 & NA & 172.47 & NA & 137.286 & 110.334 & 109.159 & 121.876 & 107.016 \\
\hline STO \& L2 & $740-G$ & H2 13 & NA & 173.86 & NA & 138.376 & 111.426 & 109.431 & 122.949 & 107.65: \\
\hline STD 224 & $740-G$ & H2 15 & NA & NA & NA & NA & NA & NA & ith & bi: \\
\hline STD 224 & $740-G$ & W2 74 & $N A$ & NA & NA & NA & NA & HA & $\mathrm{Nh}$ & 4 \\
\hline STO 224 & $740-6$ & W2 176 & NA & NA & NA & NA & NA & $N A$ & $K A$ & ' \\
\hline STO VC 1000 & $740-G$ & H2 16 & 1000 & NA & NA & NA & NA & HA & NA & \\
\hline STD VC 1000 & $740-G$ & H2 175 & 959 & NA & NA & NA & NA & $\mathrm{HA}$ & $\mathrm{NA}$ & \\
\hline
\end{tabular}

Table 5. Analyses of "I" Standards: b) Chlorinated Hydrocarbons 
WESTINGHOUSE SAVANNAH RIVER SITE .....

DECEMBER, 1990 (W3) STAMDARDS .....

..... GAS CONCENTRATIONS (PPMV) .....

STANDARD

GC

ETHYL M\&P-

0 -

name area cycle pentane hexane heptahe benzene octane toluene nonake benzene Xylene Xylene decane

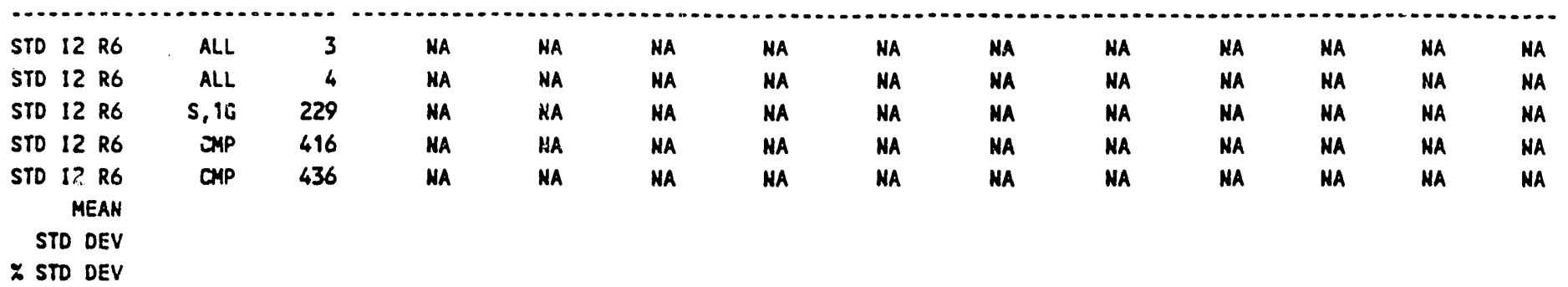

\begin{tabular}{|c|c|c|c|c|c|c|c|c|c|c|c|c|c|c|}
\hline STO & $12 R 5$ & ALL & 5 & NA & NA & NA & MA & NA & NA & NA & NA & NA & NA & NA \\
\hline STO & 12 RS & ALL & 6 & HA & NA & MA & NA & NA & NA & NA & NA & NA & NA & NA \\
\hline STD & I2 RS & $s$ & 200 & NA & NA & NÁ & NA & NA & MA & NA & NA & NA & NA & NA \\
\hline STO & $12 R 5$ & 5,10 & 228 & MA & NA & NA & NA & NA & NA & NA & NA & NA & NA & MA \\
\hline STO & $12 R 5$ & $1 \mathrm{GL}$ & 270 & NA & NA & NA & NA & NA & HA & NA & NA & NA & NA & NA \\
\hline STO & I2 RS & CMP & 301 & NA & NA & NA & NA & NA & MA & NA & NA & NA & NA & NA \\
\hline STO & $12 R 5$ & CMP & 415 & HA & NA & NA & NA & NA & NA & HA & MA & MA & NA & NA \\
\hline STO & $12 R 5$ & CMP & 435 & MA & NA & NA & NA & NA & NA & NA & NA & NA & NA & NA \\
\hline STO & $12 \mathrm{RS}$ & CMP & 449 & NA & NA & NA & NA & MA & NA & NA & MA & NA & NA & NA \\
\hline
\end{tabular}

STO DEV

* STO DEV

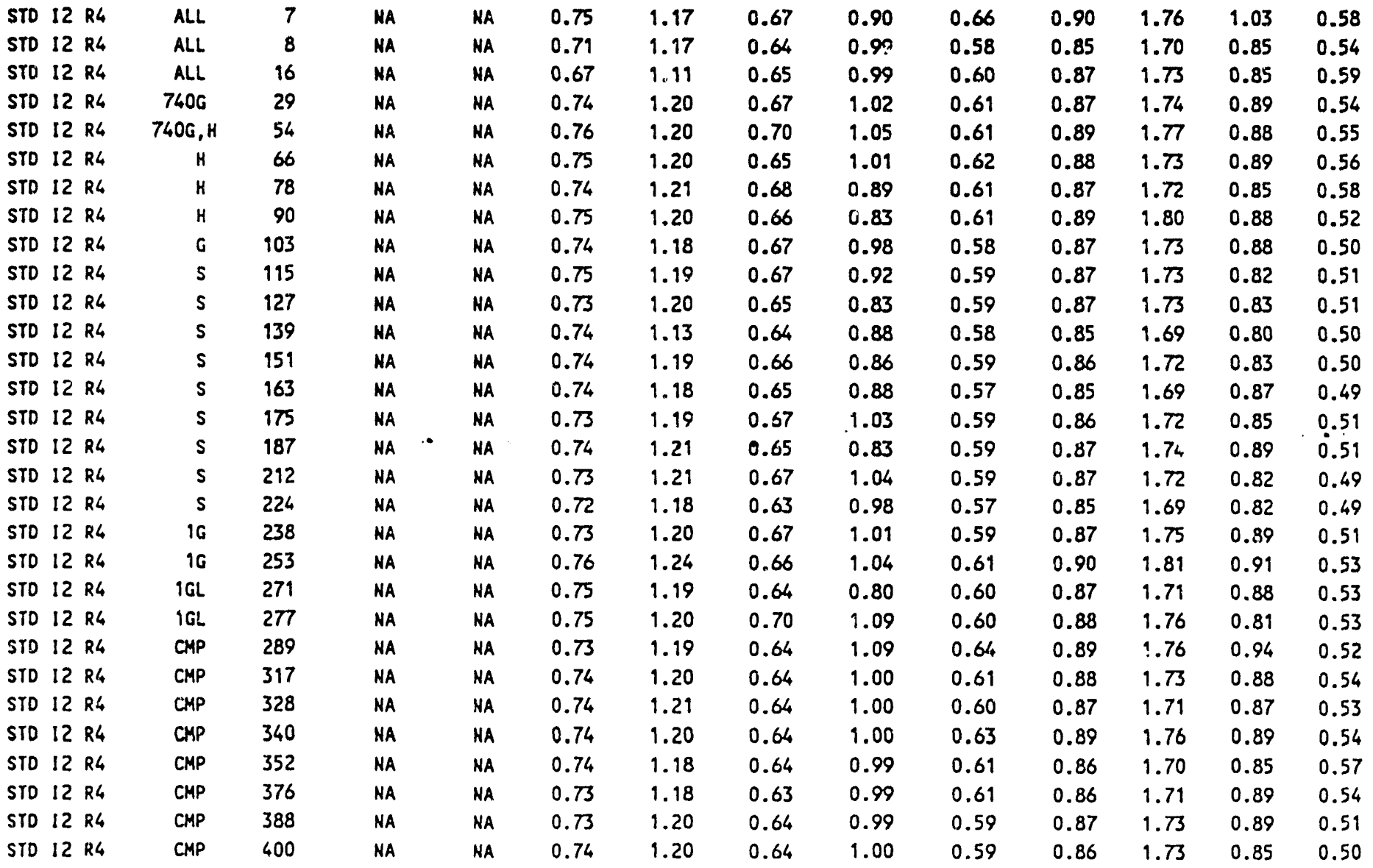




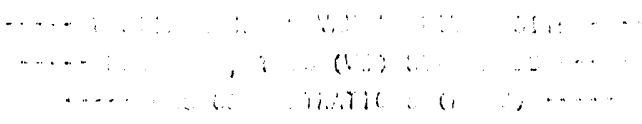

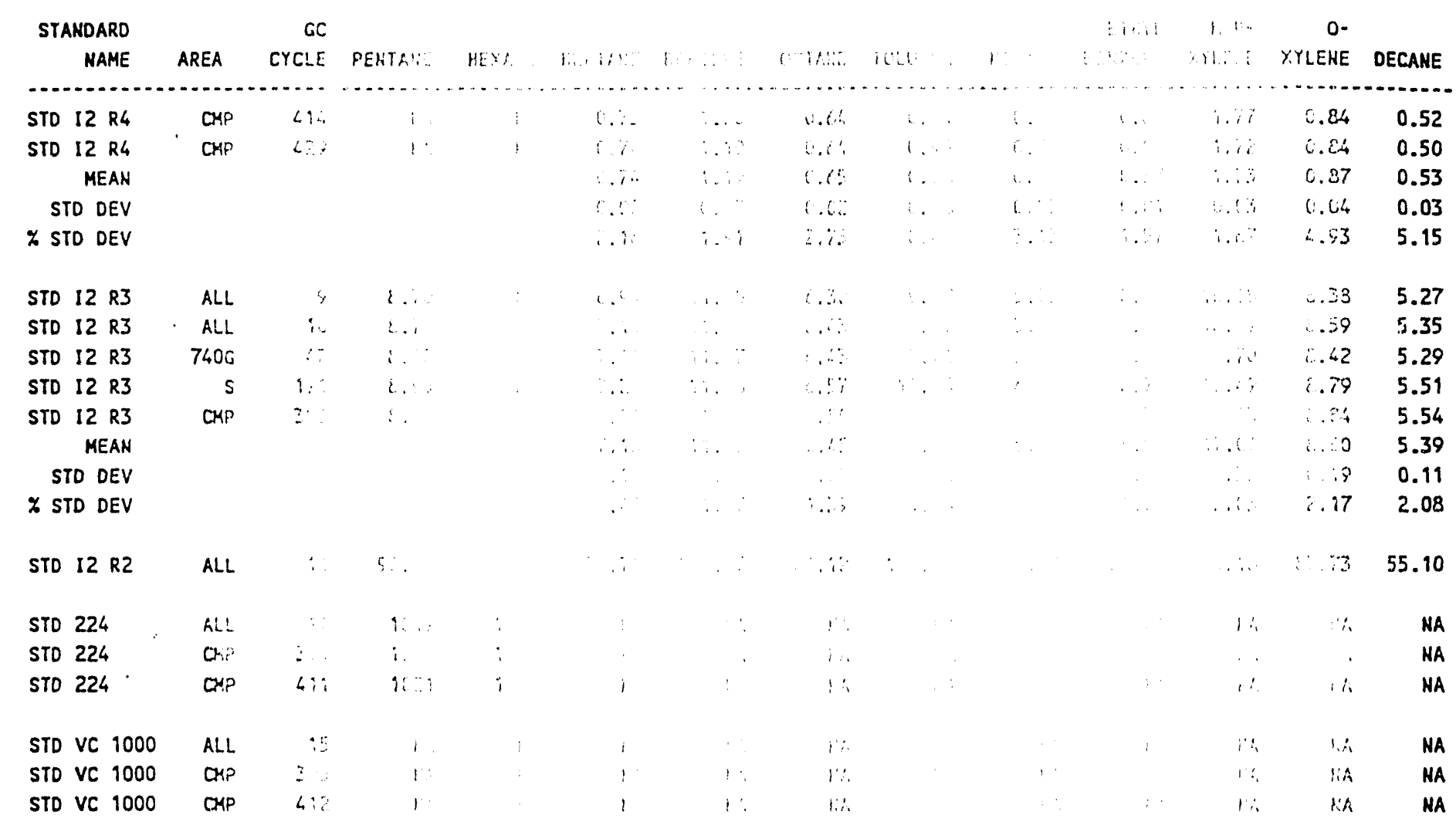


$\ldots . .$.
WESTINGHOUSE SAVAHNAH RIVER SITE
$\ldots . .$.

\begin{tabular}{|c|c|c|c|c|c|c|c|c|c|c|}
\hline $\begin{array}{r}\text { STANDARD } \\
\text { NAME }\end{array}$ & AREA & $\begin{array}{r}G C \\
\text { CYCLE }\end{array}$ & $\begin{array}{r}\text { VINYL } \\
\text { CHLORIDE }\end{array}$ & $\begin{array}{r}\text { METHYLENE } \\
\text { CHLORIDE }\end{array}$ & $\begin{array}{l}\text { TRANS } 1,2 \\
\text { DICHLC } 20 \\
\text { ETHYLENE }\end{array}$ & $\begin{array}{r}\text { CHLORO } \\
\text { FORM }\end{array}$ & $\begin{array}{l}111 \text { TRI } \\
\text { CHLORO } \\
\text { ETHANE }\end{array}$ & $\begin{array}{r}\text { CARBON } \\
\text { TETRA } \\
\text { CHLORIDE }\end{array}$ & $\begin{array}{l}\text { TRICLORO } \\
\text { ETHYLENE }\end{array}$ & $\begin{array}{r}\text { TETRA } \\
\text { CHLORO } \\
\text { ETHYLENE }\end{array}$ \\
\hline STO 12 R6 & ALL & 3 & NA & NA & NA & 0.010 & 0.008 & 0.011 & 0.009 & 0.010 \\
\hline STD 12 R6 & ALL & 4 & NA & NA & NA & 0.011 & 0.009 & 0.011 & 0.010 & 0.010 \\
\hline STD I2 R6 & $S, 1 G$ & 229 & NA & MA & NA & 0.015 & 0.012 & 0.012 & 0.011 & 0.011 \\
\hline STD 12 R6 & CMP & 416 & NA & MA & NA & 0.015 & 0.013 & 0.013 & 0.011 & 0.011 \\
\hline STD I2 R6 & CMP & 436 & NA & NA & NA & 0.017 & 0.014 & 0.013 & 0.011 & 0.011 \\
\hline MEAN & & & & & & 0.014 & 0.011 & 0.012 & 0.010 & 0.011 \\
\hline STO DEV & & & & & & 0.003 & 0.002 & 0.001 & 0.001 & 0.000 \\
\hline \% STD DEV & & & & & & 19.510 & 20.671 & 7.454 & 7.692 & 4.622 \\
\hline STO 12 R5 & ALL & 5 & NA & NA & 0.16 & 0.185 & 0.144 & 0.109 & 0.165 & 0.103 \\
\hline STD I2 R5 & ALL & 6 & NA & NA & 0.17 & 0.187 & 0.146 & 0.109 & 0.165 & 0.103 \\
\hline STD 12 R5 & $s$ & 200 & NA & NA & 0.17 & 0.182 & 0.142 & 0.130 & 0.156 & 0.108 \\
\hline STO I2 R5 & $S, 1 G$ & 228 & NA & NA & 0.17 & 0.185 & 0.138 & 0.132 & 0.157 & 0.109 \\
\hline STO 12 RS & IGL & 270 & NA & NA & 0.17 & 0.188 & 0.146 & 0.135 & 0.159 & 0.111 \\
\hline STO I2 R5 & CMP & 301 & NA & NA & 0.17 & 0.188 & 0.151 & 0.135 & 0.159 & 0.108 \\
\hline STD 12 R5 & CMP & 415 & HA & NA & 0.18 & 0.190 & 0.153 & 0.137 & 0.158 & 0.109 \\
\hline STO I2 R5 & CMP & 435 & NA & NA & 0.19 & 0.197 & 0.157 & 0.141 & 0.163 & 0.112 \\
\hline STO I2 R5 & CMP & 449 & NA & NA & 0.19 & 0.201 & 0.162 & 0.145 & 0.169 & 0.194 \\
\hline MEAN & & & & & 0.17 & 0.189 & 0.149 & 0.130 & 0.161 & 0.109 \\
\hline STD DEV & & & & & 0.01 & 0.006 & 0.007 & 0.012 & 0.004 & 0.003 \\
\hline \% STO DEV & & & & & 4.51 & 3.028 & 4.834 & 9.327 & 2.595 & 3.224 \\
\hline STD $12 R 4$ & ALL & 7 & NA & 1.63 & 1.36 & 1.278 & 1.026 & 1.094 & 1.142 & 1.037 \\
\hline STO I2 R4 & ALL & 8 & NA & 1.61 & 1.35 & 1.273 & 1.022 & 1.086 & 1.135 & 1.025 \\
\hline STO I2 R4 & $A L L$ & 16 & NA & 2.61 & 1.40 & 1.383 & 1.099 & 1.211 & 1.170 & 1.069 \\
\hline STD 12 R4 & $740 \mathrm{G}$ & 29 & NA & 1.56 & 1.49 & 1.408 & 1.121 & 1.315 & 1.211 & 1.243 \\
\hline STO $12 R 4$ & $740 G, H$ & 54 & NA & 1.60 & 1.53 & 1.467 & 1.187 & 0.992 & 1.238 & 1.000 \\
\hline STO $12 R 4$ & H & 66 & NA & 2.21 & 1.48 & 1.436 & 1.175 & 1.497 & 1.206 & 1.217 \\
\hline STO 12 R4 & H & 78 & NA & 3.29 & 1.46 & 1.429 & 1.179 & 1.501 & 1.176 & 1.119 \\
\hline STD $12 R 4$ & $H$ & 90 & NA & 2.13 & 1.60 & 1.552 & 1.273 & 1.348 & 1.294 & 1.148 \\
\hline STD 12 R4 & G & 103 & NA & 5.49 & 1.57 & 1.551 & 1.289 & 1.087 & 1.277 & 1.033 \\
\hline STO I2 R4 & $\mathbf{s}$ & 115 & NA & 2.01 & 1.61 & 1.563 & 1.287 & 1.358 & 1.289 & 1.140 \\
\hline STD $12 R 4$ & s & 127 & NA & 4.71 & 1.58 & 1.553 & 1.292 & 1.092 & 1.270 & 1.027 \\
\hline STD $12 R 4$ & s & 139 & NA & 9.47 & 1.55 & 1.549 & 1.299 & 1.089 & 1.251 & 0.987 \\
\hline STO $12 R 4$ & s & 151 & NA & 4.49 & 1.61 & 1.595 & 1.331 & 1.123 & 1.302 & 1.060 \\
\hline STD 12 R4 & $s$ & 163 & NA & 5.96 & 1.59 & 1.588 & 1.333 & 1.119 & 1.287 & 1.033 \\
\hline STD 12 RG & $s$ & 175 & NA & 3.96 & 1.63 & 1.599 & 1.342 & 1.131 & 1.300 & 1.065 \\
\hline STO I2 R4 & s & 187 & NA & 4.54 & 1.63 & 1.612 & 1.360 & 1.148 & 1.301 & 1.068 \\
\hline STD $12 R 4$ & $s$ & 212 & NA & 4.78 & 1.36 & 1.307 & 1.062 & 1.114 & 1.143 & 1.023 \\
\hline STO 12 R4 & $\mathbf{s}$ & 224 & NA & 4.22 & 1.36 & 1.289 & 1.041 & 1.093 & 1.133 & 1.008 \\
\hline STO 12 R4 & 10 & 238 & NA & 5.36 & 1.36 & 1.308 & 1.054 & 1.115 & 1.143 & 1.025 \\
\hline STO 12 R4 & $1 G$ & 253 & NA & 4.13 & 1.42 & 1.355 & 1.094 & 1.160 & 1.191 & 1.104 \\
\hline STD 12 R4 & $1 G L$ & 271 & $N A$ & 2.07 & 1.36 & 1.295 & 1.057 & 1.095 & 1.123 & 1.019 \\
\hline STD I 2 R4 & IGL & 277 & NA & 2.08 & 1.38 & 1.328 & 1.088 & 1.143 & 1.165 & 1.068 \\
\hline STO $12 R 4$ & CMP & 289 & NA & 2.40 & 1.39 & 1.323 & 1.086 & 1.136 & 1.156 & 1.049 \\
\hline STD $12 R 4$ & CMP & 317 & $N A$ & 2.59 & 1.40 & 1.345 & 1.109 & 1.157 & 1.165 & 1.061 \\
\hline STD $12 R 4$ & CMP & 328 & $N A$ & 2.20 & 1.42 & 1.357 & 1.119 & 1.166 & 1.179 & 1.070 \\
\hline STO $12 R 4$ & CMP & 340 & NA & 2.57 & 1.41 & 1.351 & 1.118 & 1.161 & 1.165 & 1.060 \\
\hline STO 12 R4 & CMP & 352 & NA & 3.91 & 1.37 & 1.342 & 1.116 & 1.154 & 1.148 & 1.028 \\
\hline STD $12 R 4$ & CMP & 376 & NA & 2.91 & 1.39 & 1.345 & 1.119 & 1.160 & 1.155 & 1.046 \\
\hline STO $12 R 4$ & CMP & 388 & NA & 3.17 & 1.42 & 1.363 & 1.133 & 1.174 & 1.169 & 1.070 \\
\hline STD $12 R 4$ & CMP & 400 & NA & 4.97 & 1.41 & 1.369 & 1.145 & 1.181 & 1.172 & 1.067 \\
\hline
\end{tabular}

Table 6. Analyses of "I2" Standards: b) Chlorinated Hydrocarbons 


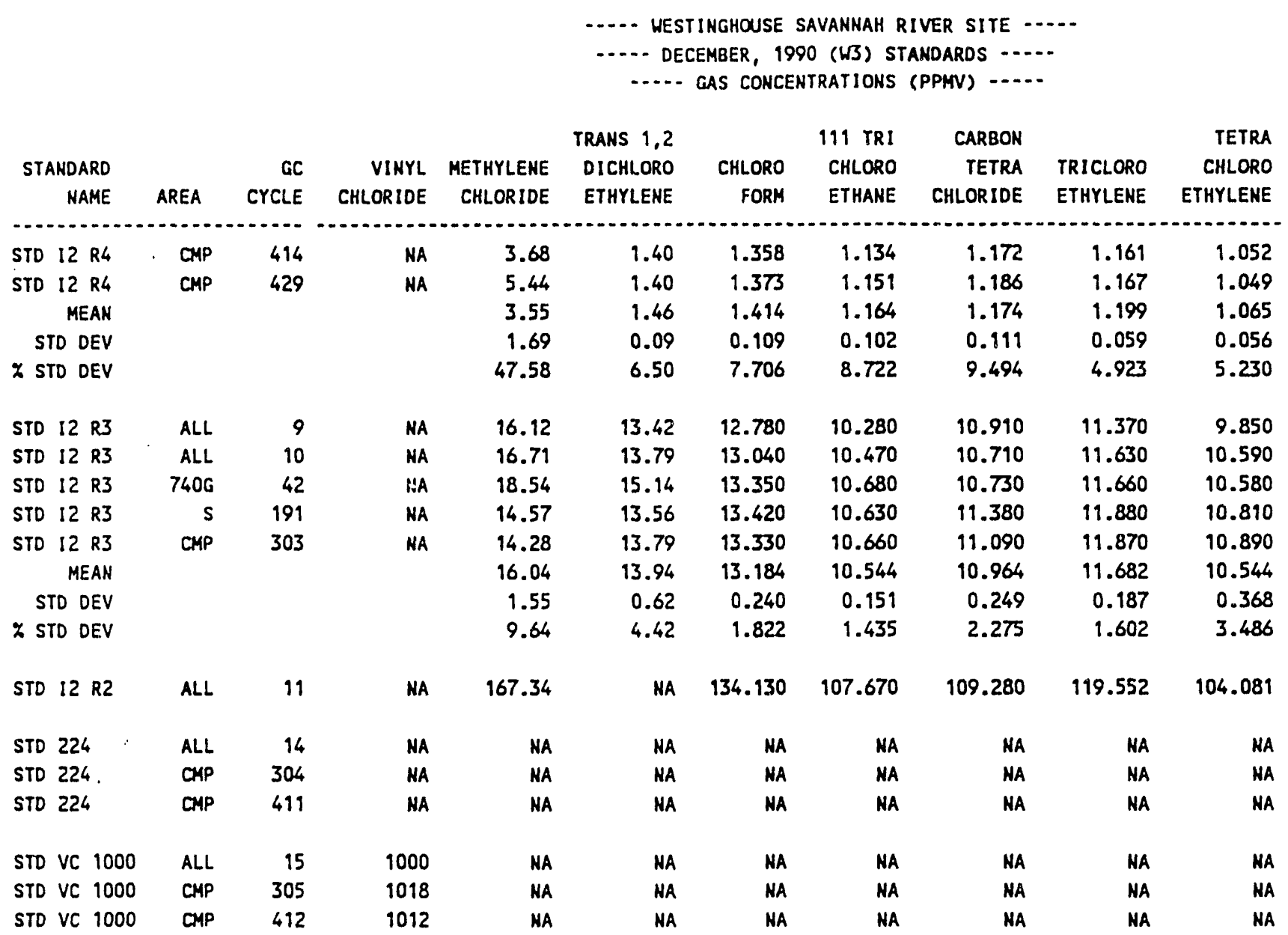

Table 6. Analyses of "I2" Standards: b) Chlorinated Hydrocarbons 
.... JUNE, 1999 (W9) STANDARDS ....

-... GAS CONCENTRATIONS (PPHV) .....

STANDARD

GC

ETHYL M\&P- ONAME cycle pentane hexame heptane benzene octane toluene nonane benzene xylene xyleme decane

\begin{tabular}{|c|c|c|c|c|c|c|c|c|c|c|c|c|c|}
\hline STO J2 R6 & ALL & wo 8 & NA & NA & NA & NA & MA & NA & HA & NA & MA & NA & NA \\
\hline STD J2 R6 & - ALL & H9 17 & NA & NA & NA & NA & NA & MA & NA & NA & NA & NA & NA \\
\hline STD J2 R6 & $080-30 G$ & W9 61 & MA & NA & NA & NA & HA & MA & MA & MA & NA & NA & NA \\
\hline STD J2 R6 & $080-01 R$ & W9 133 & NA & NA & NA & NA & NA & HA & NA & NA & NA & MA & NA \\
\hline $\begin{array}{r}\text { STO J2 R6 } \\
\text { MEAN } \\
\text { STD DEV } \\
\text { \% STD DEV }\end{array}$ & $904-83 G$ & W9 182 & NA & NA & HA & NA & NA & NA & MA & NA & NA & MA & MA \\
\hline STD J2 R5 & ALL & w9 9 & NA & NA & 0.07 & 0.12 & 0.07 & 0.10 & 0.06 & 0.09 & 0.13 & 0.08 & 0.05 \\
\hline STD J2 R5 & $A L L$ & W9 18 & MA & NA & 0.06 & 0.11 & 0.06 & 0.09 & 0.07 & 0.09 & 0.17 & 0.08 & 0.06 \\
\hline STD J2 R5 & $131-4 L$ & Wo 37 & NA & NA & 0.07 & 0.11 & 0.06 & 0.09 & 0.06 & 0.08 & 0.17 & 0.09 & 0.05 \\
\hline STD J2 R5 & $080-29 G$ & 1986 & NA & NA & 0.07 & 0.11 & 0.06 & 0.09 & 0.06 & 0.08 & 0.16 & 0.08 & 0.05 \\
\hline STD J2 R5 & ALL & w9 113 & NA & NA & 0.07 & 0.11 & 0.07 & 0.09 & 0.06 & 0.07 & 0.13 & 0.09 & 0.05 \\
\hline STO J2 R5 & $904-83 G$ & W9 214 & NA & NA & 0.07 & 0.11 & 0.06 & 0.09 & 0.06 & 0.08 & 0.13 & 0.07 & 0.05 \\
\hline STD J2 R5 & $904-83 G$ & W9 286 & NA & NA & 0.08 & 0.11 & 0.06 & 0.09 & 0.06 & 0.08 & 0.13 & 0.08 & 0.06 \\
\hline STO J2 R5 & $904-83 G$ & w9 360 & NA & NA & 0.07 & 0.11 & 0.06 & 0.09 & 0.06 & 0.08 & 0.18 & 0.10 & 0.05 \\
\hline STO J2 RS & $904-83 G$ & W9 384 & MA & NA & 0.07 & 0.10 & 0.06 & 0.09 & 0.05 & 0.08 & 0.14 & 0.09 & 0.06 \\
\hline STD J2 R5 & $108-4 R$ & 19432 & $\mathrm{NA}$ & NA & 0.06 & 0.10 & 0.06 & 0.09 & 0.05 & 0.08 & 0.15 & 0.07 & 0.04 \\
\hline MEAN & & & & & 0.07 & 0.11 & 0.06 & 0.09 & 0.06 & 0.08 & 0.15 & 0.08 & 0.05 \\
\hline STD DEV & & & & & 0.01 & 0.01 & 0.00 & 0.00 & 0.00 & 0.00 & 0.02 & 0.01 & 0.00 \\
\hline X STD DEV & & & & & 8.20 & 4.85 & 2.89 & 3.66 & 7.62 & 6.00 & 12.28 & 11.77 & 9.27 \\
\hline STO J2 R4 & $A L L$ & W9 10 & NA & MA & 0.72 & 1.19 & 0.66 & 1.00 & 0.61 & 0.88 & 1.72 & 0.87 & 0.55 \\
\hline STD J2 R4 & ALL & w9 19 & NA & HA & 0.72 & 1.19 & 0.63 & 0.97 & 0.57 & 0.83 & 1.64 & 0.83 & 0.50 \\
\hline STO J2 R4 & $131-4 L$ & wo 25 & NA & NA & 0.72 & 1.17 & 0.64 & 0.98 & 0.57 & 0.85 & 1.70 & 0.85 & 0.53 \\
\hline STO J2 R4 & $080-30 \mathrm{G}$ & wo 49 & NA & MA & 0.71 & 1.17 & 0.63 & 0.94 & 0.54 & 0.81 & 1.65 & 0.84 & 0.47 \\
\hline STD J2 R4 & $080-29 G$ & w9 74 & NA & NA & 0.73 & 1.20 & 0.65 & 0.98 & 0.58 & 0.86 & 1.74 & 0.87 & 0.55 \\
\hline STO J2 R4 & $080-29 G$ & w9 98 & HA & MA & 0.72 & 1.17 & 0.62 & 0.95 & 0.56 & 0.82 & 1.61 & 0.79 & 0.52 \\
\hline STD J2 R4 & $A L L$ & W9 111 & NA & NA & 0.70 & 1.14 & 0.62 & 0.95 & 0.54 & 0.80 & 1.57 & 0.82 & 0.46 \\
\hline STO $J 2 R 4$ & $080-01 R$ & W9 121 & MA & NA & 0.72 & 1.17 & 0.62 & 0.96 & 0.54 & 0.81 & 1.61 & 0.77 & 0.45 \\
\hline STD J2 R4 & $740-G$ & w9 145 & NA & NA & 0.72 & 1.18 & 0.63 & 0.94 & 0.55 & 0.81 & 1.58 & 0.78 & 0.46 \\
\hline STD J2 R4 & $740-G$ & W9 170 & NA & NA & 0.73 & 1.20 & 0.65 & 0.98 & 0.59 & 0.85 & 1.66 & 0.84 & 0.55 \\
\hline STD J2 R4 & $904-83 G$ & W9 194 & NA & NA & 0.70 & 1.16 & 0.62 & 0.94 & 0.56 & 0.81 & 1.60 & 0.79 & 0.48 \\
\hline STD J2 R4 & $904-83 G$ & w9 226 & HA & NA & 0.73 & 1.19 & 0.64 & 0.97 & 0.56 & 0.84 & 1.64 & 0.86 & 0.51 \\
\hline STD J2 R4 & $904-83 G$ & w9 250 & NA & NA & 0.72 & 1.18 & 0.63 & 0.97 & 0.58 & 0.84 & 1.68 & 0.79 & 0.51 \\
\hline STD $12 R 4$ & $904-83 G$ & W9 274 & NA & NA & 0.71 & 1.16 & 0.64 & 0.96 & 0.56 & 0.82 & 1.62 & 0.82 & 0.52 \\
\hline STD J2 R4 & $904-830$ & w9 300 & MA & NA & 0.71 & 1.17 & 0.63 & 0.96 & 0.55 & 0.82 & 1.63 & 0.82 & 0.49 \\
\hline STO J2 R4 & $904-83 G$ & w9 324 & NA & NA & 0.73 & 1.17 & 0.64 & 0.97 & 0.57 & 0.84 & 1.71 & 0.86 & 0.54 \\
\hline STD J2 R4 & $904-83 G$ & w9 348 & HA & NA & 0.71 & 1.16 & 0.63 & 0.95 & 0.56 & 0.82 & 1.61 & 0.87 & 0.51 \\
\hline STO J2 R4 & $904-836$ & w9 372 & HA & NA & 0.73 & 1.20 & 0.64 & 1.10 & 0.55 & 0.83 & 1.61 & 0.86 & 0.50 \\
\hline STO J2 R4 & $904-83 G$ & w9 393 & NA & NA & 0.65 & 1.05 & 0.56 & 1.14 & 0.47 & 0.70 & 1.38 & 0.63 & 0.42 \\
\hline STD J2 R4 & $904-496$ & W9 420 & NA & NA & 0.69 & 1.13 & 0.61 & 0.92 & 0.53 & 0.78 & 1.53 & 0.76 & 0.44 \\
\hline MEAN & & & & & 0.71 & 1.17 & 0.63 & 0.98 & 0.56 & 0.82 & 1.62 & 0.82 & 0.50 \\
\hline STO DEV & & & & & 0.02 & 0.03 & 0.02 & 0.05 & 0.03 & 0.04 & 0.08 & 0.06 & 0.04 \\
\hline * STD DEV & & & & & 2.53 & 2.80 & 3.24 & 5.32 & 4.76 & 4.34 & 4.68 & 6.76 & 7.58 \\
\hline STD J2 R3 & $A L L$ & Ho 11 & 9.13 & NA & 7.18 & 11.77 & 6.47 & 9.90 & 5.89 & 8.59 & 17.09 & 8.61 & 5.40 \\
\hline STO J2 R3 & $A L L$ & wo 110 & 8.98 & NA & 6.95 & 11.46 & 6.16 & 9.44 & 5.40 & 8.02 & 15.73 & 8.02 & 4.63 \\
\hline STO J2 R3 & $740-G$ & K9 158 & 8.93 & NA & 6.99 & 11.50 & 6.27 & 9.58 & 5.66 & 8.28 & 16.41 & 8.29 & 5.10 \\
\hline STO J2 R3 & $904-49 G$ & W9 238 & 8.85 & NA & 6.95 & 11.45 & 6.21 & 9.50 & 5.57 & 8.19 & 16.19 & 8.23 & 4.96 \\
\hline STO J2 R3 & $904-49 G$ & W9 312 & 8.91 & NA & 6.92 & 11.40 & 6.09 & 9.32 & 5.34 & 7.93 & 15.51 & 7.90 & 4.55 \\
\hline STO J2 R3 & $904-496$ & W9 408 & 8.57 & NA & 6.72 & 11.07 & 5.92 & 9.05 & 5.17 & 7.68 & 15.02 & 7.60 & 4.44 \\
\hline
\end{tabular}

Table 7. Analyses of "J2" Standards: a) Hydrocarbons 


\begin{tabular}{|c|c|c|c|c|c|c|c|c|c|c|c|c|c|}
\hline $\begin{array}{r}\text { STANDARD } \\
\text { NAME }\end{array}$ & AREA & $\begin{array}{r}\text { GC } \\
\text { CYCLE }\end{array}$ & PENTANE & HEXANE & HEPTANE & BENZENE & OCTANE & TOLUENE & NONANE & $\begin{array}{r}\text { ETHYL } \\
\text { BENZENE }\end{array}$ & $\begin{array}{r}\text { M\&P- } \\
\text { XYLENE }\end{array}$ & $\begin{array}{r}0- \\
\text { XYLENE }\end{array}$ & DECANE \\
\hline STD J2 R3 & $108-4 R$ & W9 443 & 8.64 & NA & 6.82 & 11.25 & 6.13 & 9.37 & 5.57 & 8.14 & 16.20 & 8.18 & 5.10 \\
\hline MEAN & . & & & & 6.93 & 11.41 & 6.18 & 9.45 & 5.51 & 8.12 & 16.02 & 8.12 & 4.88 \\
\hline STD DEV & & & & & 0.13 & 0.20 & 0.16 & 0.24 & 0.22 & 0.26 & 0.62 & 0.30 & 0.32 \\
\hline ₹ STO DEV & & & & & 1.92 & 1.78 & 2.53 & 2.55 & 3.94 & 3.26 & 3.88 & 3.64 & 6.65 \\
\hline STO J2 R2 & ALL & W9 12 & 91.52 & NA & 72.00 & 118.02 & 64.92 & 99.27 & 59.05 & 86.14 & 171.38 & 86.34 & 52.98 \\
\hline STD J2 R2 & ALL & W9 21 & 89.37 & NA & 69.86 & 114.72 & 62.42 & 95.48 & 55.66 & 81.94 & 161.38 & 82.03 & 48.22 \\
\hline STD 220 & ALL & W9 7 & 98.85 & 100.18 & MA & HA & NA & MA & NA & HA & NA & NA & HA \\
\hline STO 220 & ALL & W9 16 & 98.76 & 99.81 & $M A$ & $M A$ & NA & NA & NA & NA & NA & HA & NA \\
\hline sto 220 & ALL & Wo 109 & 97.52 & 97.85 & MA & NA & HA & NA & HA & NA & MA & NA & KA \\
\hline STO 220 & $904-83 G$ & W9 208 & 96.85 & 97.38 & MA & NA & MA & NA & NA & NA & HA & NA & NA \\
\hline STD 220 & $108-4 R$ & 11944 & 96.43 & 96.97 & NA & NA & NA & HA & NA & HA & NA & NA & NA \\
\hline STO VC -10.3 & $A L L$ & W9 5 & HA & NA & NA & NA & NA & NA & MA & NA & NA & $N A$ & NA \\
\hline STD VC- 10.3 & ALL & W9 14 & HA & NA & HA & HA & HA & HA & MA & HA & MA & MA & NA \\
\hline STD VC -10.3 & ALL & W9 107 & NA & NA & NA & NA & NA & NA & NA & HA & HA & MA & MA \\
\hline STO VC -10.3 & $904-83 G$ & Wo 207 & HA & NA & NA & MA & NA & NA & NA & NA & NA & NA & HA \\
\hline STD VC- 10.3 & $904-49 G$ & W9 396 & NA & HA & NA & HA & NA & HA & NA & MA & NA & NA & MA \\
\hline STD VC- 1027 & ALL & Wo 6 & NA & NA & NA & NA & NA & $\mathrm{NA}$ & NA & NA & NA & NA & NA \\
\hline STD VC- 1027 & ALL & WO 15 & NA & NA & NA & NA & NA & MA & MA & HA & MA & NA & MA \\
\hline SYD VC- 1027 & $A L L$ & W9 108 & NA & NA & NA & HA & NA & NA & NA & NA & NA & NA & MA \\
\hline
\end{tabular}


- JUNE, 1991 (W9) STANDARDS -...

-... GAS CONCENTRATIONS (PPMV) ....

\begin{tabular}{|c|c|c|c|c|c|c|c|c|c|c|}
\hline $\begin{array}{r}\text { STANDARD } \\
\text { NAME }\end{array}$ & AREA & $\begin{array}{r}\text { GC } \\
\text { CYCLE }\end{array}$ & $\begin{array}{r}\text { VINYL } \\
\text { CHLORIDE }\end{array}$ & $\begin{array}{l}\text { METHYLENE } \\
\text { CHLORIDEE }\end{array}$ & $\begin{array}{l}\text { TRANS } 1.2 \\
\text { DICHLORO } \\
\text { ETHYLENE }\end{array}$ & $\begin{array}{r}\text { CHLORO } \\
\text { FORM }\end{array}$ & $\begin{array}{l}111 \text { TRI } \\
\text { CHLORO } \\
\text { ETHANE }\end{array}$ & $\begin{array}{r}\text { CARBON } \\
\text { TETRA } \\
\text { CHLORIDE }\end{array}$ & $\begin{array}{l}\text { TRICLORO } \\
\text { ETHYLENE }\end{array}$ & $\begin{array}{r}\text { TETRA } \\
\text { CHLORO } \\
\text { ETHYLENE }\end{array}$ \\
\hline & & & & & & & & & 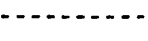 & - \\
\hline STD J2 R6 & ALL & W9 8 & NA & NA & HA & 0.013 & 0.011 & 0.019 & 0.012 & 0.010 \\
\hline STO J2 R6 & ALL & Wg 17 & NA & NA & NA & 0.013 & 0.009 & 0.011 & 0.012 & 0.010 \\
\hline STD J2 R6 & $080-30 \mathrm{G}$ & W9 61 & NA & NA & NA & 0.013 & 0.009 & 0.019 & 0.012 & 0.010 \\
\hline STO J2 R6 & $080-01 R$ & w9 133 & NA & NA & NA & 0.012 & 0.009 & 0.091 & 0.011 & 0.009 \\
\hline STD J2 R6 & $904-83 G$ & wo 182 & NA & NA & HA & 0.012 & 0.009 & 0.011 & 0.011 & 0.010 \\
\hline MEAN & & & & & & 0.013 & 0.009 & 0.011 & 0.012 & 0.010 \\
\hline STD DEV & & & & & & 0.000 & 0.001 & 0.000 & 0.000 & 0.000 \\
\hline \% STD DEV & & & & & & 3.888 & 8.511 & 0.000 & 4.223 & 4.082 \\
\hline STD J2 R5 & ALL & W9 9 & NA & NA & 0.14 & 0.131 & 0.105 & 0.109 & 0.117 & 0.103 \\
\hline STO J2 R5 & ALL & Wo 18 & NA & NA & 0.14 & 0.131 & 0.104 & 0.110 & 0.118 & 0.101 \\
\hline STD J2 R5 & $131-4 L$ & Wo 37 & NA & NA & 0.15 & 0.134 & 0.108 & 0.112 & 0.119 & 0.101 \\
\hline STD J2 R5 & $080-29 G$ & W9 86 & NA & NA & 0.14 & 0.131 & 0.105 & 0.112 & 0.118 & 0.100 \\
\hline STD J2 R5 & ALL & W9 113 & NA & NA & 0.14 & 0.124 & 0.101 & 0.107 & 0.112 & 0.094 \\
\hline STO J2 R5 & $904-83 G$ & W9 214 & NA & NA & 0.15 & 0.125 & 0.116 & 0.109 & 0.114 & 0.096 \\
\hline STO J2 R5 & $904-83 G$ & W9 286 & NA & NA & 0.15 & 0.126 & 0.109 & 0.111 & 0.116 & 0.098 \\
\hline STO J2 R5 & $904-83 G$ & w9 360 & NA & NA & 0.15 & 0.125 & 0.109 & 0.110 & 0.115 & 0.097 \\
\hline STO J2 R5 & $904-83 G$ & W9 384 & NA & NA & 0.15 & 0.124 & 0.103 & 0.109 & 0.116 & 0.099 \\
\hline STO J2 R5 & $108-4 R$ & W9 432 & NA & NA & 0.14 & 0.116 & 0.098 & 0.102 & 0.109 & 0.092 \\
\hline MEAN & & & & & 0.14 & 0.127 & 0.106 & 0.109 & 0.115 & 0.098 \\
\hline STD DEV & & & & & 0.00 & 0.005 & 0.005 & 0.003 & 0.003 & 0.003 \\
\hline \% STD DEV & & & & & 2.65 & 3.718 & 4.280 & 2.423 & 2.400 & 3.148 \\
\hline STD J2 R4 & ALL & W9 10 & NA & 1.62 & 1.34 & 1.308 & 1.046 & 1.086 & 1.174 & 1.041 \\
\hline STD J2 R4 & ALL & W9 19 & NA & 1.60 & 1.36 & 1.294 & 1.040 & 1.078 & 1.152 & 1.007 \\
\hline STD J2 R4 & $131-4 L$ & W9 25 & NA & 1.65 & 1.39 & 1.313 & 1.055 & 1.094 & 1.167 & 1.019 \\
\hline STD J2 R4 & $080-30 G$ & W9 49 & NA & 1.67 & 1.37 & 1.297 & 1.061 & 1.096 & 1.139 & 0.981 \\
\hline STD J2 R4 & $080-29 G$ & W9 74 & NA & 1.57 & 1.40 & 1.306 & 1.071 & 1.107 & 1.169 & 1.025 \\
\hline STD J2 R4 & $080-296$ & W9 98 & NA & 1.64 & 1.38 & 1.276 & 1.050 & 1.087 & 1.138 & 0.991 \\
\hline STO J2 R4 & ALL & W9 111 & NA & 1.60 & 1.34 & 1.233 & 1.022 & 1.059 & 1.122 & 0.963 \\
\hline STO J2 R4 & $080-01 R$ & W9 121 & $N A$ & 1.64 & 1.37 & 1.257 & 1.045 & 1.085 & 1.133 & 0.969 \\
\hline STO J2 R4 & $740-6$ & W9 145 & NA & 1.67 & 1.45 & 1.296 & 1.098 & 1.120 & 1.166 & 0.995 \\
\hline STD $12 R 4$ & $740-6$ & w9 170 & NA & 1.67 & 1.48 & 1.297 & 1.093 & 1.123 & 1.192 & 1.036 \\
\hline STD J2 R4 & $904-83 G$ & Wy 194 & NA & 1.67 & 1.41 & 1.255 & 1.051 & 1.092 & 1.146 & 0.990 \\
\hline STD J2 R4 & $904-836$ & W9 226 & NA & 1.67 & 1.48 & 1.286 & 1.092 & 1.123 & 1.183 & 1.019 \\
\hline STD J2 R4 & $904-83 G$ & W9 250 & NA & 1.65 & 1.49 & 1.277 & 1.082 & 1.115 & 1.189 & 1.026 \\
\hline STO J2 R4 & $904-83 G$ & W9 274 & NA & 1.69 & 1.45 & 1.259 & 1.067 & 1.104 & 1.165 & 1.007 \\
\hline STD J2 R4 & $904-830$ & w9 300 & NA & 1.63 & 1.45 & 1.258 & 1.066 & 1.103 & 1.165 & 1.005 \\
\hline STO J2 R4 & $904-83 G$ & W9 324 & NA & 1.70 & 1.47 & 1.261 & 1.069 & 1.105 & 1.186 & 1.031 \\
\hline STO J2 R4 & $904-83 G$ & H9 348 & NA & 1.63 & 1.45 & 1.250 & 1.058 & 1.100 & 1.165 & 1.003 \\
\hline STD J2 R4 & $904-83 G$ & W9 372 & NA & 1.71 & 1.46 & 1.255 & 1.070 & 1.110 & 1.168 & 0.999 \\
\hline STD J2 R4 & $904-83 G$ & W9 393 & NA & 1.58 & 1.29 & 1.141 & 1.000 & 1.018 & 1.027 & 0.865 \\
\hline STD J2 R4 & $904-49 G$ & W9 420 & NA & 1.63 & 1.39 & 1.190 & 1.014 & 1.059 & 1.131 & 0.961 \\
\hline MEAN & & & & 1.64 & 1.41 & 1.265 & 1.058 & 1.093 & 1.154 & 0.997 \\
\hline STD DEV & & & & 0.04 & 0.05 & 0.041 & 0.025 & 0.025 & 0.035 & 0.038 \\
\hline$\%$ STD DEV & & & & 2.24 & 3.85 & 3.21 & 2.36 & 2.26 & 3.05 & 3.79 \\
\hline STD JZ R3 & ALL & W9 11 & NA & 16.41 & 13.64 & 13.100 & 10.500 & 10.901 & 11.700 & 10.291 \\
\hline STD J2 R3 & ALL & W9 110 & NA & 16.19 & 13.66 & 12.464 & 10.312 & 10.741 & 11.316 & 9.599 \\
\hline STD J2 R3 & $740-G$ & W9 158 & $N A$ & 16.11 & 13.91 & 12.611 & 10.439 & 10.680 & 11.607 & 10.019 \\
\hline STD J2 R3 & $904-49 G$ & W9 238 & $N A$ & 16.19 & 13.91 & 12.451 & 10.372 & 10.491 & 11.587 & 9.998 \\
\hline STD J2 R3 & $904-49 \mathrm{G}$ & W9 312 & NA & 16.14 & 13.89 & 12.421 & 10.399 & 10.489 & 11.426 & 9.442 \\
\hline STD J2 R3 & $904-49 G$ & W9 408 & NA & 15.85 & 13.62 & 11.663 & 9.815 & 9.912 & 11.194 & 9.440 \\
\hline
\end{tabular}

Table 7. Analyses of "J2" Standards: b) Chlorinated Hydrocarbons 
JUNE, 1991 (W9) STANDARDS -....

.... GAS CONCENTRATIONS (PPMV) ....

\begin{tabular}{|c|c|c|c|c|c|c|c|c|c|c|}
\hline $\begin{array}{r}\text { STANDARD } \\
\text { NAME }\end{array}$ & AREA & $\begin{array}{r}\text { GC } \\
\text { CYCLE }\end{array}$ & $\begin{array}{r}\text { VINYL } \\
\text { CHLORIDE }\end{array}$ & $\begin{array}{r}\text { METHYLENE } \\
\text { CHLORIDE }\end{array}$ & $\begin{array}{l}\text { TRANS } 1,2 \\
\text { DICHLORO } \\
\text { ETHYLENE }\end{array}$ & $\begin{array}{r}\text { CHLORO } \\
\text { FORM }\end{array}$ & $\begin{array}{r}111 \text { TRI } \\
\text { CHLORO } \\
\text { ETHANE }\end{array}$ & $\begin{array}{r}\text { CARBON } \\
\text { TETRA } \\
\text { CHLORIDE }\end{array}$ & $\begin{array}{l}\text { TRICLORO } \\
\text { ETHYLENE }\end{array}$ & $\begin{array}{r}\text { TETRA } \\
\text { CHLORO } \\
\text { ETHYLENE }\end{array}$ \\
\hline & & & & & & & & & & - -2 \\
\hline STD J2 R3 & $108-4 R$ & W9 443 & NA & 15.96 & 13.94 & 11.882 & 9.960 & 10.064 & 11.543 & 9.571 \\
\hline MEAN & & & & 16.12 & 13.80 & 12.370 & 10.257 & 10.468 & 11.482 & 9.766 \\
\hline STO DEV & & & & 0.17 & 0.14 & 0.439 & 0.243 & 0.334 & 0.165 & 0.310 \\
\hline \% STD DEV & & & & 1.03 & 0.99 & 3.55 & 2.37 & 3.19 & 1.44 & 3.17 \\
\hline STD J2 R2 & $A L L$ & W9 12 & NA & 164.57 & 99.31 & 131.799 & 105.789 & 109.312 & 117.380 & 103.240 \\
\hline STD J2 R2 & ALL & W9 21 & NA & 161.31 & 97.51 & 129.530 & 103.775 & 104.813 & 113.081 & 98.709 \\
\hline STD 220 & ALL & W9 7 & HA & NA & NA & HA & NA & NA & NA & NA \\
\hline STD 220 & ALL & W9 16 & NA & NA & NA & NA & NA & NA & NA & NA \\
\hline STD 220 & ALL & W9 109 & NA & NA & NA & NA & NA & NA & NA & NA \\
\hline STO 220 & $904-83 G$ & W9 208 & NA & NA & NA & NA & NA & NA & NA & NA \\
\hline STD 220 & $108-4 R$ & W9 444 & NA & NA & NA & NA & NA & NA & NA & NA \\
\hline STD $V C-10.3$ & ALL & Wo 5 & 10.1 & MA & NA & NA & NA & NA & NA & NA \\
\hline STD VC- 10.3 & ALL & W9 14 & 10.5 & NA & NA & NA & NA & NA & NA & NA \\
\hline STD VC- 10.3 & ALL & W9 107 & 10.0 & NA & NA & NA & NA & NA & NA & HA \\
\hline STO VC-10.3 & $904-83 G$ & W9 207 & 9.9 & HA & NA & HA & $\mathrm{NA}$ & $N A$ & NA & MA \\
\hline STO VC- 10.3 & $904-49 G$ & W9 396 & 10.1 & NA & NA & NA & NA & NA & NA & NA \\
\hline
\end{tabular}


LIGHT HYROCARBOA STANDARD ANALYSIS FOR 740-G LAND

.... LIGHT HYROCARBON STANDARD ANALYSIS FOR 740-G LANDFILL ....

\begin{tabular}{|c|c|c|c|c|c|c|c|c|c|}
\hline \multicolumn{2}{|c|}{ STANDARD } & $\begin{array}{r}\text { DATE } \\
\text { ANALYZED }\end{array}$ & $\begin{array}{r}\text { METHANE } \\
\text { PPB }\end{array}$ & $\begin{array}{r}\text { ETHANE } \\
\text { PPB }\end{array}$ & $\begin{array}{r}\text { PROPANE } \\
\text { PPB }\end{array}$ & $\begin{array}{r}\text { 1-BUTANE } \\
\text { PPB }\end{array}$ & $\begin{array}{r}\text { N-BUTANE } \\
\text { PPB }\end{array}$ & $\begin{array}{r}\text { ETHYLENE } \\
\text { PPB }\end{array}$ & $\begin{array}{c}\text { PROPYLENE } \\
\text { PPB }\end{array}$ \\
\hline \multicolumn{10}{|c|}{. $\therefore . . .}$. \\
\hline STO & & $11-11-90$ & 10060 & 1006 & 1003 & 988 & 937 & 1011 & 1012 \\
\hline STD & M & $11-11-90$ & 10088 & 1013 & 1007 & 1000 & 966 & 1017 & 1018 \\
\hline STD & $M$ & $11-11-90$ & 10229 & 1010 & 1016 & 977 & 922 & 1016 & 1016 \\
\hline STD & M & $11-11-90$ & 10049 & 1009 & 1004 & 987 & 954 & 1010 & 1008 \\
\hline STD & $M$ & $11-12-90$ & 10056 & 1007 & 1003 & 1001 & 979 & 1008 & 1004 \\
\hline STD & M & $11-12-90$ & 10014 & 1005 & 1002 & 996 & 973 & 1006 & 1010 \\
\hline STD & M & $11-13-90$ & 10311 & 1027 & 1032 & 1018 & 998 & 1031 & 1032 \\
\hline STD & M & $11-13-90$ & 10342 & 1041 & 1043 & 1047 & 1040 & 1049 & 1035 \\
\hline STD & M & $11-13-90$ & 10323 & 1035 & 1034 & 1029 & 995 & 1041 & 1042 \\
\hline STD & $M$ & $11-13-90$ & 10421 & 1049 & 1046 & 1053 & 1030 & 1055 & 1049 \\
\hline STD & M & $11-13-90$ & 10446 & 1047 & 1050 & 1041 & 1019 & 1055 & 1046 \\
\hline STD & $M$ & $11-15-90$ & 10000 & 1000 & 1000 & 1000 & 1000 & 1000 & 1000 \\
\hline STD & $M$ & $11-15-90$ & 9916 & 1005 & 993 & 994 & 976 & 1006 & 1003 \\
\hline STO & $M$ & $11-15-90$ & 9898 & 1000 & 994 & 993 & 977 & 995 & 1001 \\
\hline STD & M & $11-16-90$ & 9926 & 994 & 990 & 989 & 977 & 993 & 992 \\
\hline STO & M & $11-16-90$ & 9859 & 994 & 988 & 992 & 980 & 986 & 992 \\
\hline STD & $M$ & $11-16-90$ & 9809 & 991 & 985 & 1005 & 1025 & 986 & 995 \\
\hline STD & M & $11-16-90$ & 9937 & 998 & 993 & 994 & 996 & 997 & 993 \\
\hline STD & M & $11-17-90$ & 9927 & 994 & 991 & 986 & 975 & 992 & 992 \\
\hline STD & M & $11-17-90$ & 9837 & 991 & 985 & 1012 & 1037 & 987 & 990 \\
\hline STD & M & $11-17-90$ & 9884 & 989 & 983 & 985 & 972 & 988 & 987 \\
\hline STO & $M$ & $11-17-90$ & 9858 & 987 & 984 & 976 & 952 & 983 & 990 \\
\hline STD & M & $11-17-90$ & 9943 & 994 & 995 & 986 & 991 & 1003 & 999 \\
\hline STO & M & $11-18-90$ & 9982 & 993 & 992 & 976 & 954 & 991 & 998 \\
\hline STD & $M$ & $11-18-90$ & 9826 & 989 & 983 & 983 & 977 & 982 & 984 \\
\hline STD & M & $11-18-90$ & 10685 & 1095 & 1174 & 1058 & 983 & 1911 & 1186 \\
\hline STD & $M$ & $12-10-90$ & 9562 & 958 & 958 & 908 & 841 & 995 & 1008 \\
\hline STD & M & $12-10-90$ & 9563 & 958 & 958 & 912 & 844 & 996 & 1011 \\
\hline STD & M & $12-10-90$ & 10000 & 1000 & 1000 & 1000 & 1000 & 1000 & 1000 \\
\hline STD & $M$ & $12-10-90$ & 10147 & 1018 & 1016 & 1031 & 1061 & 1019 & 1014 \\
\hline STD & $M$ & $12-10-90$ & 10110 & 1018 & 1017 & 1003 & 988 & 1016 & 1015 \\
\hline STD & $M$ & $12-10-90$ & 10112 & 1016 & 1017 & 1052 & 1095 & 1014 & 1022 \\
\hline \multirow[t]{4}{*}{ STO } & M & $12-10-90$ & 10078 & 1010 & 1011 & 1010 & 1000 & 1012 & 1010 \\
\hline & & AVERAGE & 10036 & 1007 & 1007 & 999 & 982 & 980 & 983 \\
\hline & & STD DEV & 234 & 25 & 36 & 32 & 49 & 175 & 177 \\
\hline & & \% STD DEV & 2.33 & 2.52 & 3.61 & 3.24 & 5.02 & 17.88 & 18.03 \\
\hline STD & 224 & $11-11-90$ & 1045500 & 1058200 & 1027900 & NA & 1004800 & NA & NA \\
\hline STD & 224 & $11-12-90$ & 1036100 & 1045900 & 1015400 & NA & 1021800 & HA & NA \\
\hline STO & 224 & $11-12-90$ & 1028500 & 1037100 & 1007100 & MA & 1066700 & HA & NA \\
\hline STD & 224 & $11-13-90$ & 1040600 & 1050300 & 1020700 & NA & 1030900 & NA & NA \\
\hline STD & 224 & $11-13-90$ & 1064600 & 1074400 & 1043500 & NA & 1054500 & NA & NA \\
\hline STD & 224 & $11-13-90$ & 998520 & 1003100 & 971300 & NA & 1004500 & NA & NA \\
\hline STD & 224 & $11-17-90$ & 981370 & 982290 & 949890 & NA & 987410 & NA & NA \\
\hline \multirow[t]{4}{*}{ STD } & 224 & $11-18-90$ & 1018300 & 1027900 & 997360 & NA & 974470 & NA & NA \\
\hline & & AVERAGE & 1026686 & 1034899 & 1004144 & & 1018135 & & \\
\hline & & STD DEV & 24985 & 28049 & 28703 & & 29732 & & \\
\hline & & \% STD DEV & 2.43 & 2.71 & 2.86 & & 2.92 & & \\
\hline
\end{tabular}

Table 8. Light Hydrocarbon Analyses of All Standards "M" and " 224 " 
This page intentionally left blank. 
WESTINGHOUSE SAVANNAH RIVER SITE

MINIMUM DETECTION LEVELS (W2,W3, W9 DATA SETS) --

- AREA REJECT $=40$, CONCENTRATIONS IN PPMV

\begin{tabular}{rrr}
$\begin{array}{r}\text { COMPOUND } \\
\text { NAME }\end{array}$ & $\begin{array}{r}\text { IDEAL } \\
\text { M.D.L. }\end{array}$ & $\begin{array}{r}\text { ACTUAL } \\
\text { M.D.L. }\end{array}$ \\
\hline VINYL CHLORIDE & 0.10 & 1.0 \\
PENTANE & 0.040 & 0.070 \\
HEXANE & 0.033 & 0.070 \\
METHYLENE CHLORIDE & 0.03 & 1.0 \\
TRANS 1,2 DICHLORO & 0.011 & 0.020 \\
CHLOROFORM & 0.00019 & 0.005 \\
III TRICHLOROETHANE & 0.00007 & 0.005 \\
CARBON TETRACHLORIDE & 0.00004 & 0.005 \\
HEPTANE & 0.026 & 0.070 \\
BENZENE & 0.032 & 0.070 \\
TRICHLOROETHYLENE & 0.00015 & 0.005 \\
OCIANE & 0.023 & 0.070 \\
TOLUENE & 0.028 & 0.070 \\
TETRACHLOROETHYLENE & 0.00005 & 0.005 \\
NONANE & 0.021 & 0.070 \\
ETHYI BENZENE & 0.024 & 0.070 \\
MEP-XYLENE & 0.025 & 0.070 \\
O-XYLENE & 0.024 & 0.070 \\
DECANE & 0.020 & 0.070
\end{tabular}

Table 9. Minimum Detection Levels 
This page intentionally left blank. 
SAVAHHAH RIVER SITE -...-

SOIL GAS SYSTEM BLANKS - - LANDFILL DATA DIRECTORIES -...

..... GAS CONCENTRATIONS (PPHV) .....

SAMPLE

NAME

740-0................

740-G SYS BLK 1

740-G SYS BLK 2

740-G SYS BLK 3

740-G SYS BLK 4

740-G SYS BLK 5

$740-G$ SYS BLK 6

740-G SYS BLK 7

740-G SYS BLK 8

740-G SYS BLK 9

740-G SYS BLK 10

740-G SYS BLK 11

740-G SYS BLK 12

740-G SYS BLK 13

$740-G$ SYS BLK 14

740-G SYS BLK 15

740-G SYS BLK 16

$740-G$ SYS BLK 17

$740-G$ SYS BLK 18

740-G SYS BLK 19

740-G SYS BLK 20

740-G SYS BLK 21

740-G SYS BLK 22

740-G SYS BLK 23

740-G SYS BLK 24

740-G SYS BLK 25

740-G SYS BLK 26

$740-G$ SYS BLK 27

740-G SYS BLK 28

740-G SYS BLK 29

740-G SYS BLK 30

740-G SYS BLK 31

740-G SYS BLK 32

740-G SYS BLK 33

740-G SYS BLK 34

AVERAGE
STD DEV
AVG+2STD DEV

ETHYL M\&P- Opentane hexane heptane benzene octane toluene honame benzene Xylene Xylene decane

\begin{tabular}{|c|c|c|c|c|c|c|c|c|c|c|}
\hline ND & ND & ND & NO & ND & ND & ND & ND & ND & ND & NO \\
\hline ND & ND & ND & ND & ND & ND & 0.035 & ND & ND & NO & 0.023 \\
\hline NO & ND & ND & ND & ND & 0.053 & ND & ND & ND & ND & NO \\
\hline ND & ND & ND & ND & ND & 0.027 & 0.034 & ND & 0.027 & ND & 0.024 \\
\hline ND & ND & HD & ND & ND & ND & ND & ND & ND & ND & ND \\
\hline NO & ND & ND & ND & ND & ND & ND & ND & ND & ND & ND \\
\hline 0.054 & ND & ND & ND & ND & 0.046 & NO & 0.029 & 0.075 & ND & 0.027 \\
\hline 0.055 & 0.083 & 0.059 & ND & 0.085 & 0.084 & 0.063 & 0.030 & 0.118 & 0.026 & 0.080 \\
\hline ND & NO & ND & ND & ND & 0.032 & ND & 0.028 & 0.088 & ND & 0.019 \\
\hline 0.100 & 0.059 & NO & ND & NO & 0.074 & NO & 0.047 & 0.130 & 0.039 & 0.041 \\
\hline 0.098 & 0.194 & ND & 0.051 & 0.049 & 0.308 & 0.024 & 0.086 & 0.300 & 0.067 & ND \\
\hline NO & NO & ND & ND & ND & ND & ND & 0.028 & 0.076 & NO & ND \\
\hline ND & ND & NO & ND & ND & 0.047 & ND & 0.032 & 0.079 & ND & ND \\
\hline ND & 0.043 & ND & ND & NO & 0.084 & 0.066 & 0.064 & 0.134 & NO & 0.058 \\
\hline ND & ND & ND & ND & ND & 0.041 & ND & 0.028 & 0.082 & 0.033 & 0.025 \\
\hline 0.056 & 0.038 & ND & ND & ND & 0.024 & NO & NO & 0.058 & ND & ND \\
\hline ND & ND & ND & ND & ND & ND & ND & ND & 0.056 & NO & ND \\
\hline ND & ND & ND & ND & NO & ND & ND & ND & 0.037 & NO & ND \\
\hline ND & ND & ND & ND & ND & ND & ND & ND & 0.059 & ND & ND \\
\hline NO & NO & ND & ND & ND & ND & ND & ND & 0.042 & HD & NO \\
\hline ND & ND & NO & ND & ND & NO & ND & ND & 0.052 & No & ND \\
\hline NO & ND & NO & ND & ND & ND & ND & NO & 0.042 & ND & ND \\
\hline ND & ND & ND & ND & ND & ND & 0.029 & ND & 0.026 & ND & 0.054 \\
\hline ND & NO & ND & ND & NO & ND & ND & ND & 0.029 & ND & 0.051 \\
\hline ND & ND & ND & ND & NO & NO & ND & ND & 0.169 & 0.100 & 0.079 \\
\hline ND & ND & ND & ND & ND & ND & NO & ND & 0.034 & ND & 0.031 \\
\hline NO & NO & NO & ND & ND & ND & ND & ND & 0.032 & ND & ND \\
\hline ND & ND & NO & NO & ND & 0.100 & ND & NO & ND & ND & 0.043 \\
\hline NO & NO & NO & NO & 0.226 & NO & ND & NO & 0.036 & ND & 0.034 \\
\hline NO & ND & ND & ND & ND & ND & ND & NO & 0.025 & ND & KO \\
\hline ND & NO & ND & ND & ND & ND & NO & MD & ND & ND & ND \\
\hline NO & ND & ND & ND & ND & ND & ND & ND & NO & ND & ND \\
\hline ND & ND & ND & ND & NO & ND & ND & ND & ND & ND & NO \\
\hline NO & ND & ND & ND & ND & ND & ND & ND & ND & ND & ND \\
\hline 0.045 & 0.040 & 0.027 & 0.033 & 0.032 & 0.045 & 0.025 & 0.029 & 0.060 & 0.028 & 0.029 \\
\hline 0.014 & 0.028 & 0.006 & 0.003 & 0.036 & 0.050 & 0.011 & 0.013 & 0.056 & 0.015 & 0.016 \\
\hline 0.073 & 0.097 & 0.038 & 0.039 & 0.103 & 0.144 & 0.046 & 0.054 & 0.172 & 0.057 & 0.062 \\
\hline
\end{tabular}


-...- SAVANHAH RIVER SITE .....

SOIL GAS SYSTEM BLANKS -- LANDFILL DATA DIRECTORIES -..-

....- GAS CONCENTRATIONS (PPMV) .....

SAMPLE

MAME

(1...................

740-G SYS BLK

740-G SYS BLK 2

740-G SYS BLK 3

$740-G$ SYS BLK 4

740-G SYS BLK 5

740-G SYS BLK 6

740-G SYS BLK 7

$740-G$ SYS BLK 8

$740-G$ SYS BLK 9

$740-G$ SYS BLK 10

740-G SYS BLK 11

740-G SYS BLK 12

740-G SYS BLK 13

$740-G$ SYS BLK 14

740-G SYS BLK 15

740-G SYS BLK 16

740-G SYS BLK 17

740-G SYS BLK 18

740-G SYS BLK 19

$740-0$ SYS BLK 20

740-G SYS BLK 21

740-G SYS BLK 22

740-G SYS BLK 23

740-G SYS BLK 24

740-G SYS BLK 25

$740-G$ SYS BLK 26

740-G SYS BLK 27

740-G SYS BLK 28

740-G SYS BLK 29

740-G SYS BLK 70

740-G SYS BLK 31

740-G SYS BLK 32

740-G SYS BLK 33

740-G SYS BL.K 34

\section{AVERAGE \\ STD DEV}

AVG+2STD DEV
TRANS 1,2
VINYL METHYLENE DICHLORO

CHLORIDE CHLORIDE ETHYLENE

NO NO

ND

ND

ND

ND

0.121

0.102

0.191

0.248

0.451

0.169

0.185

NO

0.120

0.156

0.147

0.147

NO

ND

ND

0.123

ND

0.414

0.250

0.727

0.309

ND

0.246

2.172

2.591

ND

0.614

0.890

0.854

0.366

0.551

1.467

0.272
0.218
0.707

0.272
0.218

0.378

ND

0.495

ND

0.354

ND

0.489

ND

0.798

0.350

0.332

0.567

0.620

0.370

0.309

ND

0.264

ND

0.307

0.011

0.462

NO
0.567

ND

ND

0.239

0.250

0.655

0.279

0.231

0.269

0.330

\begin{tabular}{|c|c|}
\hline ND & ND \\
\hline ND & ND \\
\hline ND & ND \\
\hline ND & ND \\
\hline ND & ND \\
\hline ND & ND \\
\hline NO & ND \\
\hline NO & NO \\
\hline KD & ND \\
\hline ND & ND \\
\hline ND & ND \\
\hline ND & ND \\
\hline NO & ND \\
\hline ND & NO \\
\hline NO & 0.00100 \\
\hline ND & ND \\
\hline ND & ND \\
\hline ND & ND \\
\hline NO & ND \\
\hline NO & ND \\
\hline ND & No \\
\hline NO & ND \\
\hline ND & ND \\
\hline ND & ND \\
\hline NO & NO \\
\hline NO & NO \\
\hline ND & NO \\
\hline ND & ND \\
\hline ND & ND \\
\hline ND & ND \\
\hline ND & NO \\
\hline 0.031 & NO \\
\hline 0.033 & ND \\
\hline 0.099 & ND \\
\hline 0.012 & 0.00021 \\
\hline 0.005 & 0.00014 \\
\hline 0.023 & 0.00049 \\
\hline
\end{tabular}

ND

111 TRI

CARBON CHLORID

ETHANE

0.00200

0.00200

0.00700

0.00300

0.00900

0.00400

0.00500

0.00100

0.00500

0.00300

0.01100

ก. 00400

0.00400

0.00600

0.00500

0.00300

0.00600

0.00400

0.00300

0.02300

0.00400

0.01000

0.00500

0.00200

0.00400

0.00200

0.00500

0.00200

0.00300

0.00300

0.00400

0.00100

0.00100

0.00200

0.00465

0.00397

0.01259

0.00004
0.00000
0.00004
0.00460

0.01047

0.02553

0.00502

0.01247

0.02996

CHLORO

ETHYLENE FYLE

CYCLE

ND ND ND WR 17

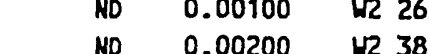

ND $0.01000 \quad 0.01300$ W2 50

ND 0.00400 N2 62

$\begin{array}{lll}0.03900 & 0.00200 \quad \text { UR } 122\end{array}$

ND $0.04400 \quad 0.01600$ W2 134

0.05300 W2 188

$0.00200 \quad 0.05300$ W2 200

$0.00300 \quad 0.00300 \quad$ W2 212

0.00100 HD H2 224

$0.00100 \quad 0.00100$ W2 236

HD H2 248

0.00100 W2 297

ND W2 309

ND W2 320

$$
0.00100 \text { W3 } 17
$$

$00100 \quad 4349$

ND W3 53

.00200 W3 102

NO 149

ND 156

w9 169

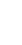

Table 10. System Blanks for All Sanitary Landfill Data Directories: b) Chlorinated Hydrocarbons 


\section{Appendix I}

Sampling Method SM1

Sampling Method SM4

Analytical Method AMI

Analytical Method AM4

Analytical Method AM8

Standard Preparation Method SP3 
This page intentionally left blank. 
SAMPLING METHOD SM1

SOIL GAS PROBE SAMPLING

\subsection{Scope and Application}

1.1 Soil gas probe samples are collected with Microseeps unique, portable soil gas sampling probe which permits access to any area. In addition, the probe allows unlimited purging between sample sites and incorporates the capability to discard the volume of air in the sample probe before the soil gas sample at each site is taken.

\subsection{Apparatus}

2.1 Soil Gas Probe: The soil gas probe consists of a $1 / 2$ inch diameter steel tube equipped with a rubber packer and a 1/8" $0 . d$. ss sample tube inserted concentrically to the bottom of the $1 / 2$ " diameter tube to minimize dead space in the sample volume. The $1 / 8$ " $0 . d$. sample tube is connected through a three way valve to a $250 \mathrm{cc}$ gas tight syringe mounted as an integral part of the sample probe. The third port of the three-way valve is terminated with a syringe needle and serves two functions: first a small amount of gas equal to or larger than the dead space volume of the $1 / 8$ " $0 . d$. sample tube is discarded through this port before each soil gas sample is taken; second, the syringe needle serves to facilitate transfer of the soil gas sample through a rubber septum into a previously evacuated sample vial, minimizing the possibility of contamination of any sample.

2.2 Slide Hammer Plunger Bar: The slide hammer plunger bar contains a $5.5 \mathrm{ft}$. $x .5$ in rod with a $9 / 16$ inch ball. Fitted over one end of the rod is a weighted slide hammer. (Heath Consultants cat\# 478).

2.3 Sample vials: The sample vials used are determined by the analytical method that is required for analysis. All vials should be free of interferences and meet the specifications described in the analytical method to be used.

\subsection{Procedure}

3.1 Prior to any sampling, the soil gas sampling probe should be purged clean of interferences, the syringe needle should be removed, and the three way valve should be in the stop flow position. 
3.2 A 9/16 inch diameter hole is created to the desired depth using the slide hammer plunger bar.

3.3 The plunger bar is removed and the sample probe is inserted into the hole. A seal should be obtained to prevent ambient air from entering the hole when the soil gas sample is removed. position.

3.4 The three way valve is turned to the probe/syringe

3.5 A $10 \mathrm{cc}$ volume of gas is drawn into the syringe.

3.6 The three way valve is turned to the syringe/needle position (needle removed as described in section 3.1 ) and the $10 \mathrm{cc}$ sample is discarded.

3.7 The three way valve is turned to the probe/syringe position and the appropriate amount of soil gas is drawn into the syringe.

3.8 The three way valve is turned to the stop flow position

3.9 The needle is attached to the probe needle fitting.

3.10 An evacuated sample vial is attached by inserting the sample vial septum onto the needle.

3.11 The three way valve is turned to the syringe/needle position. The volume of the evacuated vial will be drawn into the sample vial. Additional volume (positive pressure) can then be added by depressing the plunger and displacing the remaining volume in the syringe.

3.12 The sample vial should be quickly removed once desired volume is displaced.

3.13 A volume of approximately $50 \mathrm{cc}$ of ambient air should be drawn back through the needle.

3.14 Remove the needle.

3.15 Renove the sample probe from the hole.

3.16 Purge the sample probe clean of interferences by filling and exhausting the syringe five times before taking the next sample. 


\subsection{Sample Documentation}

4. I All samples should be labeled immediately after collection with the following information:

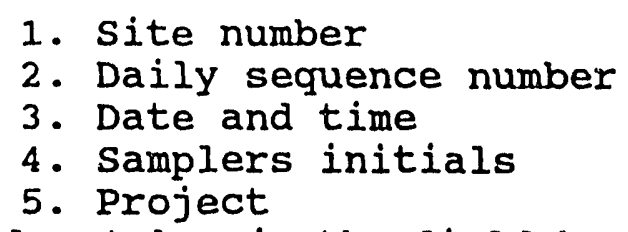

4.2 All samples taken in the field by Microseeps, Ltd. should be entered onto a Field Log Sheet. For each sample, the following entries will be made:

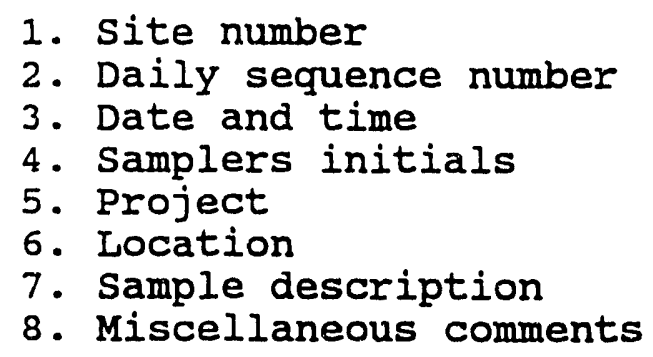

4.3 When appropriate, samples will be security sealed and chain of custody records will be maintained.

\subsection{Safety Precautions}

5.1 Do not use a mechanical device to depress the sampling syringe plunger.

5.2 Do not attempt to collect soil gas probe samples without prior knowledge of the location of underground utilities and other possible environmental hazards.

5.3 Always cap or remove the soil gas probe syringe needle when transporting or handling the soil gas probe.

\subsection{Preventive Maintenance}

6.0 The soil gas sample probe should be cleaned when necessary or at the end of each day with soap and organic free water. It is especially important to keep the gas sampling syringe clean from fine soil particles as the particles will quickly deteriorate the teflon/glass plunger seal.

6.1 The glass wool filter plug (located in the probe tip) should be changed often and the probe tip should be kept clear of soil. 
This page intentionally left blank. 


\section{GOIL PI,UG SAMPLING FOR MERCURY}

Soil plugs of 6 inches in length are collected between the depths of 12 and 18 inches. A 1.25 inch stainless steel pipe is driven 12 inches, removed from the hole and the 12 inch core discarded. The pipe is re-inserted into the hole and the 6 inch soil plug is obtained between 12 and 18 inches.

A portion of this six inch soil plug is then placed directly into a plastic sample bag and labeled. A duplicate sample is collected for every 10 sites.

A field collection $\mathrm{log}$ is maintained which lists the sample name, date and time of collection, collection sequence number, sample depth, sample type, and comments. 
This page intentionally left blank. 
ANALYTICAL METHOD AM1

\section{ANALYSIS OF $C_{1}-C_{4}$ HYDROCARBONS, HYDROGEN, HELIUM, IN SOIL GAB}

\subsection{Scope and Application}

1.1 Method AMI is used to determine the concentration of $C_{1}-C_{4}$ hydrocarbons, helium and hydrogen in soil gas samples. Specifically, Method AMI may be used to detect the following substances:

methane

ethane

ethylene

propane

propylene

i-butane

n-butane

helium

hydrogen

1.2 This method is recommended for use by, or under the supervision of, analysts experienced in the operation of gas chromatographs and in the interpretation of chromatograms.

\subsection{Summary of Method}

2.1 Analysis of the above constituents of free soil gas is accomplished with a custom chromatograph, built by Microseeps. The custom, dual detector gas chromatograph provides a sequential output from two detectors. First, the $C_{1}-C_{4}$ hydrocarbons are detected with a flame ionization detector (FID), next helium and hydrogen are detected with a thermal conductivity detector (TCD). The sample (and standard calibration gas) is flowed through the gas chromatograph and introduced into the columns by the mechanical injection of two sample loops.

\subsection{Interferences}

3.1 Contamination by carryover can occur whenever high-level and low-level samples are sequentially analyzed. A non-restricted flow of pure nitrogen from a 10 psig source should be purged for 30 seconds through the sample loops prior to all analyses.

3.2 The analyst should demonstrate the absence of contamination by carryover by analysis of the contents of the sample loops when purged with nitrogen. This demonstration should be performed prior to the 
analysis of a sample set and when carryover contamination is suspected (after high samples). In the event that 'ghost peaks' (peaks similar to previous sample) appear when a pure nitrogen sample is analyzed, measures should be taken to eliminate the carryover contamination.

3.3 Extra peaks in a chromatogram can be actual peaks from a previous run. Contamination from late eluting peaks can occur when injection to injection time is too short. The dual gas chromatograph is set up in the backflush pre-column valve arrangement to minimize this interference.

3.4 The analyst should be certain that all peaks have eluted from the previous analysis prior to analyzing any sample or standard. If samples or standard chromatograms contain suspected 'extra peaks' the sample should again be analyzed after a clean baseline is established.

\subsection{Apparatus and Materials}

4.1 Sample vials: $125 \mathrm{ml}$ glass vials (Wheaton \#223748 or equivalent). Vials should be free of hydrocarbons, helium, and hydrogen prior to use. This can be accomplished by heating to 100 degrees $C$ followed by purging with pure nitrogen.

4.2 Septums: Butyl rubber septums (Wheaton \#224154 or equivalent) may be used provided vials are capped within two weeks prior to use. other septums may be used, provided they are gas tight and do not produce interferences.

4.3 Gas Chromatograph: The chromatograph is equipped with two sets of the following: column oven, pre-column, analytical column, detector, injection port, sample valve and sample loop. The column and detector for determination of $C_{1}-C_{4}$ hydrocarbons are a granular $3 \mathrm{ft} . x 3 / 16$ in. alumina analytical column and a flame ionization detector. The alumina column is protected against contamination by heavy organics by a 3 in. $x 3 / 16$ in. pre-column which is back-flushed after butanes have entered the analytical column. This arrangement allows rapid turn-around for consecutive analyses and a clean baseline for accurate, reproducible results. The flame ionization detector is of a special design which allows considerably more sensitivity than commercially available models. In a laboratory setting, noise levels are commonly at the 500 ppt level. Although, these sensitivities are rarely realized in the field, 1 to 2 $\mathrm{ppb}$ is achievable and $5 \mathrm{ppb}$ is routine. Hydrogen and helium, are simultaneously determined using a 3 ft. $x 3 / 16$ in. molecular sieve $5 \mathrm{~A}$ $(80 / 100$ mesh) pre-column and 10 ft. $x 3 / 16$ in. analytical column connected to a thermal conductivity detector. Injection times and the signal output are sequenced so that the output of the two detectors is displayed in a single continuous chromatogram.

4.4 Data Collection: The output of the chromatograph is directed to a chart recorder and a Hewlett Packard (HP-3392A) Networking Integrator 


\subsection{Sample Preparation and Analysis}

5.1 Sample vial preparation: All sample vials should meet specifications as noted in sections 4.1 and 4.2 above. vials should be tightly capped and evacuated to a pressure of less than 100 millitorr. The vial septum should be punctured with needles of 22 gauge or smaller.

5.2 The evacuated sample vials should be filled with sample gas to a pressure of 9 psig. A positive pressure capable of delivering at least $25 \mathrm{cc}$ of sample must be available per each injection.

5.3 The pressurized sample vial is connected to the sample loops through a needle fitting and flow control valve. The flow is monitored by a flow meter connected to the out port on the gas chromatograph.

5.4 After the appropriate volume of sample (minimum $25 \mathrm{cc}$ ) has been flushed through the sample loops of the gas chromatograph, the injection valves may be activated. This is accomplished by switching on the cycle timer.

\subsection{Calibration}

6.1 The standard calibration gas should be introduced in the same manner as described in section 5.4 above. Measured peak areas are converted to concentrations in parts per million by volume using certified commercial gas standards traceable to NBS standards. (Matheson Gas Products Inc., or Scott Specialty Gases). The sample concentrations are calculated from calibration points near the concentration level of the sampie.

6.2 At the beginning of a project or sample set, standards of appropriate calibration ranges will be run at least three times or until the results agree with a percent standard deviation no greater than $10 \%$.

6.3 The instrument response (for any one subsequent standard in section 6.5 above) must not vary by more than 20\%; or any two consecutive standards must not vary by more than $15 \%$ from the mean of the three previous standards. 


\subsection{Quality control}

7.1 If the parameters set forth in section 6.3 are not met, the analytical program will be terminated until the cause is determined and a solution is effected. The cause and the solution will be recorded in the Laboratory Notebook and signed by the operater, before the analytical program is resumed.

7.2 Before and during sample analysis, instrument blanks (sample loops filled with flush nitrogen) should be analyzed to assure the absence of interferences as described in section 3.0 above.

7.3 Standards analyzed during the course of analyzing samples are averaged into the calibration table as well as being used for peak identification. All chromatograms should be examined by an experienced analyst.

7.4 Throughout analysis the soil gas samples are injected mechanically to achieve a uniform sample size from a flow directly from the soil gas sample vial which has been pressured at the time of sampling. This pressuring preserves sample integrity since any leakage is out of the vial and does not result in contamination or sample dilution.

7.5 Calibration records are generated and stored. All such records will be maintained in the laboratory during the course of the project.

\subsection{Instrument Conditions}

8. 1 Gas Chromatograph:

Injection Temp. ambient

Flame Ionization Detector Temp. ambient

Thermal Conductivity Detector Temp. 45 deg. C.

C1-C4 Oven Temp. 100 deg. C. isothermal

Helium/Hydrogen oven Temp. $45 \mathrm{deg}$. C. isothermal

Initial T.C.D. Signal Range 10

Initial F.I.D. Signal Range 10E9

Carrier Gas Regulator Pressures:

T.C.D. 40 psig.

F.I.D. 17 psig.

Hydrogen Pressure 22 psig.

Flame Air Pressure $30 \mathrm{psig.} \mathrm{(1.0} \mathrm{scfh)}$ 
ANALYTICAL METHOD AM4

ANALYSIS OF VOLATILE ORGANIC COMPOUNDS IN BOIL GAS

1.0 Scope and Application

1.1 Method AM4 is used to determine the concentration of volatile organic compounds in soil gas samples. Specifically, Method AM4 may be used to detect the following compounds:

$\begin{array}{ll}\text { pentane } & \text { benzene } \\ \text { hexane } & \text { toluene } \\ \text { heptane } & \text { m \& p-xylene } \\ \text { octane } & \text { o-xylene } \\ \text { nonane } & \text { ethyl benzene } \\ \text { methane } & \\ \text { vinyl chloride } \\ \text { l,1-dichloroethylene } \\ \text { methylene chloride } \\ \text { 1,1-dichloroethane } \\ 1,2-d i c h l o r o e t h a n e \\ \text { trans 1,2-dichloroethylene } \\ \text { chloroform } \\ \text { 1,1,1-trichloroethane } \\ \text { carbon tetrachloride } \\ \text { trichloroethylene } \\ \text { tetrachloroethylene }\end{array}$

1.2 This method is recommended for use by, or under the supervision of, analysts experienced in the operation of a gas chromatograph and in the interpretation of a chromatogram.

\subsection{Summary of Method}

The volatile organic compounds are analyzed using a Hewlett Packard Model 5890A Gas Chromatograph in conjunction with a Hewlett Packard Model 19395A Automated Headspace Sampler and an H.P. 3396A Networking Integrator. A Supelco, $60 \mathrm{M} \times 0.75 \mathrm{~mm}$ i.d. Vocol, wide bore capillary column is used in conjunction with an output splitter connected to an electron capture detector and a flame ionization detector. The integrator is interfaced to an IBM microcomputer for data storage and processing. Data transfer and analyses are facilitated using a chromatography data system (Chrom Perfect, Justice Innovations). 


\subsection{Interferences}

3.1 Contamination by carryover can occur whenever high-level and low-level samples are sequentially analyzed. While in the standby mode, the HP 19395A provides continuous flushing of the sample loop and sample valve between sample analyses. This flush flow should be maintained and the sample valve and loop should be kept heated.

3.2 The analyst should demonstrate the absence of carryover contamination by analysis of the contents of the sample loop when purged with pure nitrogen. This demonstration should be performed prior to the analysis of a sample set and when carryover contamination is suspected (after high samples). In the event that 'ghost peaks' (peaks similar to previous sample) appear when a pure nitrogen sample is analyzed measures should be taken to eliminate the carryover contamination.

3.3 Extra peaks in a chromatogram can be actual peaks from a previous run. Contamination from compounds eluting late in the chromatogram can occur when injection to injection time is too short. The HP 5890A is equipped with a temperature programmable oven which can be utilized to minimize this interference.

3.4 The analyst should be certain that all peaks have eluted from the previous analysis prior to analyzing any sample or standard. This can be accomplished by elevating the oven temperature after an analysis until such time that a clean stable baseline is obtained. If samples or standard chromatograms contain suspected 'extra peaks' the sample should be reanalyzed after a clean baseline is established.

\subsection{Materials and Equipment}

4.1 Sample vials: $22 \mathrm{ml}$ glass vials (Hewlett Packard \#93010716 or equivalent). Vials should be free of chlorinated hydrocarbons prior to use. This can be accomplished by washing and rinsing with hydrocarbon free water then heating to 100 degrees $C$ for 1 hour followed by purging with pure nitrogen.

4.2 Septums: Teflon Iined septums (Wheaton \#224168 or equivalent) may be used provided vials are capped within two weeks prior to use. Other septums may be used provided they are gas tight and do not produce interferences.

4.3 Gas Chromatograph: The Hewlett Packard 5890A Gas Chromatograph is equipped with a Supelco, $60 \mathrm{M} \times 0.75 \mathrm{~mm}$ i.d. Vocol, wide bore capillary column connected to an electron capture detector and flame ionization detector. 
4.4 Headspace Sampler: A Hewlett Packard Model 19395A Automated Headspace Sampler is used which contains a heated bath with 24 slots for headspace vials, a heated sample valve, a heated sample loop and a heated transfer line to facilitate transfer of the sample onto the column in the gas chromatograph.

4.5 Data collection: The output of the chromatograph is directed to a chart recorder and a Hewlett Packard (HP-3396A) Networking Integrator which passes the processed data to a microcomputer for data storage and/or further processing.

\subsection{Sample Preparation and Analysis}

5.1 Sample vial preparation: All sample vials should meet specifications as noted in sections 4.1 and 4.2 above. Vials should be tightly capped and evacuated to a pressure of less than 100 millitorr. The vial septum should be punctured only with needles of 22 gauge or smaller.

5.2 The evacuated sample vials should be filled with sample or standard gas to a positive gauge pressure. Sample vials should be used (filled with sample) within two weeks of preparation.

5.3 The 22 cc sample vials are placed directly into the heated headspace sampling unit for a minimum of 90 minutes prior to injection.

5.4 The headspace sampling unit is programmed to mechanically puncture the septum, transfer the sample to the heated sample loop, and mechanically inject the sample into the column flow stream via the heated transfer line.

\subsection{Standards and Calibrations}

6.1 Gas standards or liquid standards may by used to achieve calibrations. In some situations it may be necessary to use both types of standards. Certified commercial gas standards are most desirable, but may not always be available for all the compounds or for the concentration levels of the compounds of interest.

6.2 Commercial gas standards are introduced by filling a $22 \mathrm{ml}$ headspace vial with standard gas. The gas standards are placed in the vials and analyzed in the same manner as samples (as described in section 5.0). The concentrations are those certified by the manufacturer.

6.3 Liquid standard solutions are injected directly into a capped vial and allowed to vaporize. These standards are produced from high purity compounds as described in standard preparation Method SPI. The liquid standard solutions are placed in vials that meet specifications described in sections 4.1 and 4.2 . The vials 
used must be capped and be at atmospheric pxessure when the Iiquid standard is injected. The vial is then analyzed in the same manner as a sample as described in section 5.3 .

6.4 At the beginning of a project or sample set, standards of appropriate calibration ranges will be run at least three times or until the results agree with a percent standard deviation no greater than $10 \%$.

6.5 Thereafter, at least one standard will be run for every 10 samples.

6.6 The instrument response (for any one subsequent standard in section 6.5 above) must not vary by more than $20 \%$; or any two consecutive standards must not vary by more than $15 \%$ from the mean of the three previous standards.

\subsection{Quality Control}

7.1 If the parameters set forth in section 6.6 are not met the analytical program will be terminated until the cause is determined and a solution is effected. The cause and the solution will be recorded in the Laboratory Notebook and signed by the operator, before the analytical program is resumed.

7.2 Before and during sample analysis, instrument blanks (sample loops filled with flush nitrogen) should be analyzed to assure the absence of interferences as described in section 3.0 above.

7.3 Prior to the analysis of a sample set, multiple standards, at different concentration levels, should be analyzed to establish an initial calibration table. During sample analysis, standards should be run at a rate of 1 for each 10 samples.

7.4 Standards analyzed during the course of analyzing samples are compared to the calibration table as well as being used for peak identification. All chromatograms should be examined by an experienced analyst.

7.5 The soil gas sample vial is pressurized at the time of sampling. This pressure preserves sample integrity since any leakage is out of the vial and does not result in contamination or sample dilution.

7.6 The headspace sampling unit contains a heated bath as well as a heated sampling loop and transfer line. The latter two zones are continually flushed with nitrogen between sample analyses to minimize the chance of instrumental carry over. This nitrogen in the sample loop is injected periodically to check for instrument contamination. 
ANALYTICAL METHOD AM8

ANALYSIS OF MOBILE MERCURY IN SOILS

\subsection{Scope and Application}

1.1 Method AM8 is used to determine the concentration of mobile (low temperature, adsorbed) mercury in soil samples.

1.2 This method is recommended for use by, or under the. supervision of, analysts experienced in the operation of mercury analyzers and in the interpretation of mercury concentration data.

\subsection{Summary of Method}

2.1 Mobile (low temperature, adsorbed) mercury is analyzed using a Jerome Model 411 Gold Film Mercury Analyzer. A weighed soil sample (dried and sieved < 35 mesh) is placed in a silica test tube $(6 \mathrm{~mm}$ id $\times 65 \mathrm{~mm}$ ) which has the bottom inside surface coated with a thin gold film. The soil in the tube is heated at 175 degrees centigrade for 1 hour. The soil is then discarded and each tube is heated individually to 600 degrees centigrade in a resistance heater. At 600 degrees $C$, vapors in the tube are caused to flow into the mercury analyzer where mercury is adsorbed onto the calibrated gold film. This gold film changes resistance proportional to the amount of mercury adsorbed. The resistance change is converted to weight of mercury adsorbed.

\subsection{Interferences}

3.1 The analyst should demonstrate that the gold coated test tubes are mercury free before soil is added. This may be accomplished by heating each tube to 600 degrees centigrade and flowing the heated vapors in the tube into the mercury analyzer. Temperature of the tube should be kept at 600 degrees centigrade until no mercury is detected on successive analyses.

3.2 In order to prevent interferences from sulfur gases, other compounds generated when soil is heated, and dust, the analyst should ensure that Malcosorb and dust filters are connected in series with the analyzer as shown in the instrument operation manual.

3.3 The analyst should demonstrate that the system is mercury free at the beginning of the analysis of a sample set. This may be accomplished by heating an uncoated test tube to 600 degrees 
centigrade and flowing the heated vapors into the analyzer for measurement. The temperature should be maintained and the flow should be repeated until no mercury is detected on successive runs.

3.4 The analyst should regularly check the status of the gold film detector and burn of the accuinulated mercury when appropriate.

\subsection{Materials and Equipment}

4.1 Sample test tubes: fused silica (6 mm id $\times 65 \mathrm{~mm}$ ) with bottom half of inner surface coated with a thin gold film.

4.2 Heating block: aluminum block ( $12 \times 12 \times 2.5$ inches) with sixty four $5 / 16 \times 2$ inch holes for holding gold coated silica tubes.

4.3 Hot plate: Thermodyne Sybron, Model 2200

4.4 Resistance heater: Nichrome wire coil to hold one silica test tube and heat to 600 degrees centigrade.

4.5 Mercury Analyzer: Jerome Model 411 Gold Film Mercury Analyzer including Malcosorb and dust filters.

4.6 Balance: Fisher Model 50 with $+/-0.005$ gram accuracy.

\subsection{Sample Preparation and Analysis}

5.1 Sample test tube preparation: All sample test tubes should meet specifications as noted in sections 3.1 and 4.1 above.

5.2 The sample should be air dried overnight(8-10 hours), then sieved using a 35 mesh sieve. The < 35 mesh fraction should be collected, placed in a clean sample envelope and labeled.

5.3 A weighed, $0.25-0.50$ gram, portion of the dried, sieved soil sample is placed into a gold coated silica test tube and the test tube number is recorded with the sample number.

5.4 The gold coated silica test tubes containing the weighed, sieved soil samples are placed in the heating block (4.2) and heated to 175 degrees centigrade for 1 hour.

5.5 After removal from the heating block, the soil is discarded from the gold coated silica test tubes. Excess dust may be removed from the vial with compressed air at room temperature.

5.6 Each empty gold coated silica tube is heated to 600 
degrees centigrade in the resistance heater and the mercury vapor is caused to flow into the analyzer where it is absorbed on the gold film.

5.7 The change in film resistance is recorded as the number of counts which may be related to the amount of mercury absorbed.

\subsection{Standards and Calibrations}

6.1 Calibration of the mercury analyzer is achieved by injecting a known amount of mercury vapor directly into a stream of air flowing into the analyzer.

6.2 Mercury metal is maintained at known temperature inside each of six $125 \mathrm{ml}$ sample bottles which are capped with septa. is maintained.

6.3 A chart of mercury vapor pressure in air vs temperature

6.4 A $1 \mathrm{ml}$ volume of air saturated with mercury vapor at a known temperature from one or more of the $125 \mathrm{ml}$ bottles $(6.2)$ is injected into the input air flow to the analyzer and a reading is taken.

6.5 After three or more such procedures, the analyzer calibration is derived as average counts/ng mercury. Typical range is 5 - 8 counts per ng. mercury.

6.6 The analyst should ensure that each bottle of mercury is left at atmospheric pressure after calibration so that temperature and vapor pressure equilibration can occur before the next use. This is achieved by puncturing the septum with a syringe needle after several calibration volumes are removed from a bottle.

\subsection{Quality Control}

7.1 The analyzer should be calibrated before and after each set of 50 samples is analyzed.

7.2 The instrument response should not vary by more that $20 \%$ between any two successive calibrations.

7.3 One blank gold coated vial should be analyzed with every ten samples. The blank vial should be representative of background contamination that could be obtained in the sample vials.

7.4 The instrument detector status should be monitored and recorded during sample analysis. 
This page intentionally left blank. 
STANDARD PREPARATION METHOD SP3

GAS STANDARD PREPARATION FOR VOC'B

\subsection{Scope and Application}

1.1 Gas standards may be prepared from pure liquid compounds when commercial gas standards are not available. Known weights of pure compounds are placed in a $22 \mathrm{ml}$ vial under known conditions. The ideal gas law is used to calculate gas volumes for each compound. Concentrations may be calculated in parts per million volume (ppmv) or micrograms per liter air (ug/l air).

\subsection{Summary}

2.1 Equal volumes of pure liquid compounds are combined. A uL amount of the pure blend is injected into a $22 \mathrm{ml}$ headspace vial (at atmos. pressure) to produce a known concentration gas (level 1 standard). The pure compound blend is diluted in solvent so that when ul amounts of the diluted blend are injected into a $22 \mathrm{ml}$ Headspace vial (at atmos. pressure) a known concentration of gas (level 2) is produced. The pure blend is again diluted in solvent to produce other gas standards (level 3 and 4 ). Level 5 and level 6 gas standard may be produced by further diluting level 2 and level 3 blends 1000:1. For each level number increase, the weight amount of compound (ug/ul blend) decreases by a factor of 10 .

\subsection{Apparatus and Materials}

3.1 Solvent: The solvent normally used is high purity $(99+\%)$ chromatographic grade methyl alcohol. It must be determined if the solvent will interfere with or mask compounds of interest.

3.2 Pure compounds of interest: All compounds used must meet required specifications and be approved on the supplies and Materials List.

3.3 Storage of Prepared Standards: Vials (Alltech \#72716 or equivalent), Caps (Alltech \#95246, mininert valves), septa (Alltech $\# 9548$ ).

equivalent.

1 ml disposable pipettes: Corning \#7078-1CN or

$3.522 \mathrm{ml}$ Headspace Vial: Hewlett Packard \#9301-0716.

3.610 ul liquid syringe: Hamilton \#701 or equivalent. 
3.7 Pipette Dispenser, Micro-Pipex: Fisher Scientific.

\subsection{Procedure}

4.1 Add $1 \mathrm{ml}$ of each compound to a pre-cleaned $22 \mathrm{ml}$ vial using a new disposable $1 \mathrm{ml}$ pipette for each compound. The total volume of the pure blend should be greater than $9 \mathrm{ml}$. If less than 9 compounds are used then $2 \mathrm{ml}$ of each compound should be combined.

4.2 Using a disposable $1.0 \mathrm{ml}$ glass pipette, fill at least two $4.5 \mathrm{ml}$ vials with the pure blend. Immediately cap the vials with mininert valve caps. Label the vials "Pure Blend standard [I.D.] Level 1" along with the date and the preparer's initials.

4.3 Using a disposable $1.0 \mathrm{ml}$ glass pipete, fill each of three new pre-labeled $4.5 \mathrm{ml}$ vials with the following ml amounts of solvent; 3.960 (level 2), 4.355 (level 3), and 4.395 (level 4). The labels should reflect the appropriate standard I.D. and level. Cap all vials with mininert valve caps.

4.4 Using a clean $10 u l$ syringe add 4.4 ul of pure blend to the vial containing $4.395 \mathrm{ml}$ of solvent and labeled level 4 .

4.5 Using the same 10 ul syringe as in step 4.4 add 44 ul of pure blend to the vial containing 4.355 and labeled level 3 .

4.6 Using a clean $1 \mathrm{ml}$ pipette add $.44 \mathrm{ml}$ of pure blend to the vial containing $3.960 \mathrm{ml}$ of solvent and labeled level 2 .

4.7 To produce level 5 and level 6 dilute blends add $4.395 \mathrm{ml}$ of solvent to two clean prelabeled $4.5 \mathrm{ml}$ vials. For level 5 add $4.4 \mathrm{ul}$ of level 2 blend and for level 6 add 4.4 ul of level 3 blend.

4.8 Gas standards are produced by injecting ul amounts of blend (levels 1-6) into $22 \mathrm{ml}$ headspace vials containing clean air and capped with TFE lined septums. Refer to the appropriate Standard Calculations Table for actual amounts to inject into the headspace vials and resulting concentrations of each compound.

4.9 The standard calculations table is produced in spreadsheet format and contains at least the following information; solvent used, compound density and molecular weight, ul compound/lul blend, compound weight/volume of blend, gas volume of compound, weight of compound per liter of air, and the parts per million of compound by volume for each concentration level of standard. 


\subsection{Calculations}

5.1 The following formulas are used to calculate the gas concentration (vol/vol) originating from liquid (weight).

CONCENTRATION in PPMV $=$ (COMPOUND VOL/TOTAL VOL $) * 1000000$

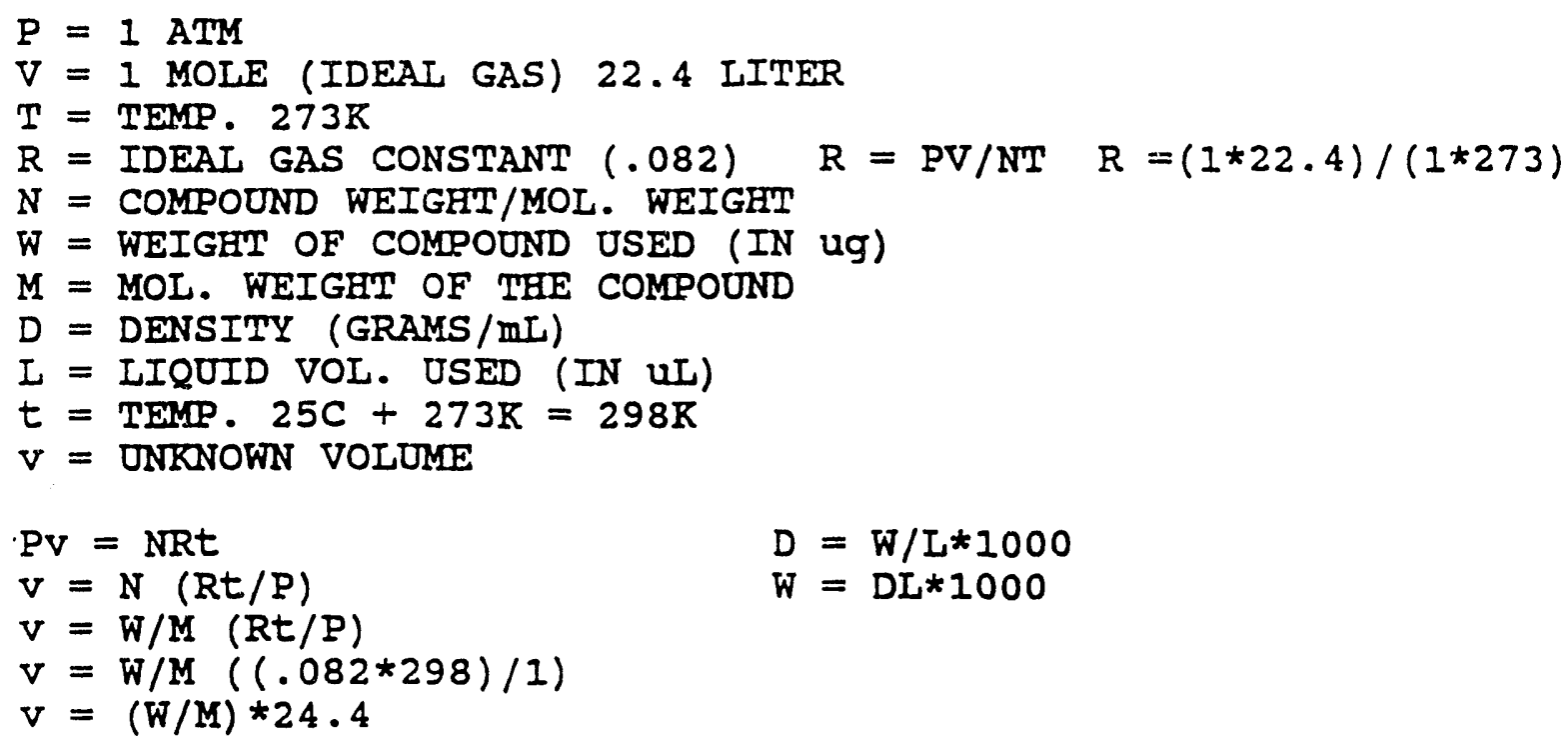

5.2 To calculate the standard gas concentrations in ug/l air the above ideal gas law formula must be used to calculate the volume of gas of the compound when added to the headspace vial. This volume is then added to the volume of the headspace vial to determine the total volume. The following formula can then be used:

CONCENTRATION (ug/I air) =

(1 Liter air/total vol) (compound weight/total volume)

\subsection{Safety Precautions}

6.1 General laboratory safety precautions should be followed when preparing standards. The safety precautions should include, but not be limited to, the following safeguards; Eye and face protection, proper clothing (gloves, shoes, etc.), proper ventilation, spill protection, and an emergency response plan.

6.2 All safety precautions as stated on the material data sheets should be followed when handling hazardous materials. 
This page intentionally left blank. 


\section{Appendix II}

Soil Gas Collection Logs Mercury Collection Logs

Waste Management Unit Worksheet 


\section{This page intentionally left blank.}


MICROSEEPS LTO.

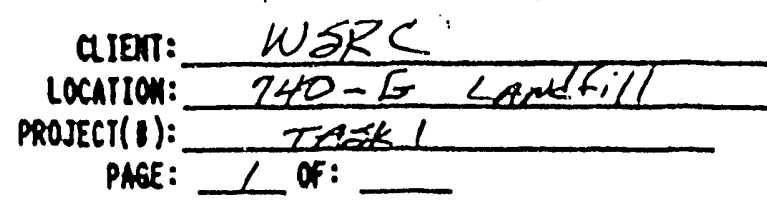

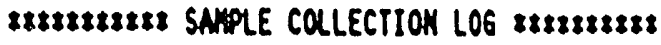

samprer make(s): R. Pirkle/A.Cayion

\begin{tabular}{|c|c|c|c|c|c|c|c|c|c|}
\hline SAMPLE IOI & OATE & IIME & SEO.1 & SAMPLE & & LE & & SAMPLE & COMMENTS \\
\hline - & $\cdots$ & & & DEPTH & 6 & 5 & 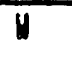 & SIZE & \\
\hline 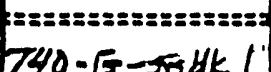 & $\begin{array}{l}:=2 x z=0 \\
1 / / 4\end{array}$ & $=:=2=3:=$ & & & $=2 x$ & & :=:=z & $x=2 \geq=2: 2$ & 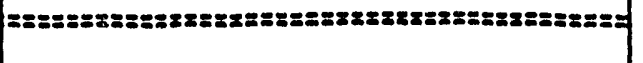 \\
\hline $740-5-504 k 1$ & 11) 14 & $\frac{1420}{1421}$ & 1 & & 2 & & & 22 & \\
\hline . $\quad-3 y \leq 2 k \leq 1$ & & 1421 & 2 & 0 & & & & 125 & \\
\hline-0001 & & 1435 & 3 & 3.0 & 1 & & & 22 & f \\
\hline-0001 & & 1436 & 4 & 3.2 & $I$ & & & 125 & \\
\hline .0002 & & 1444 & $\Sigma$ & $\therefore$ & 7 & & & 22 & 1 \\
\hline .0002 & & 1448 & 6 & & I & & & 125 & ixesik thron \\
\hline-0003 & & 1456 & 7 & & I & & & 22 & $0, z^{\prime}$ \\
\hline-0003 & & 1457 & 8 & 1 & 1 & & & $125^{\circ}$ & I \\
\hline-6004 & & 1504 & 9 & $i$ & $i$ & & & 22 & I \\
\hline-0004 & & 1505 & 0 & $i$ & $i$ & & & 125 & I \\
\hline-0005 & & 1508 & II & I & $T$ & & & 32 & $i$ \\
\hline .0005 & & 1509 & 12 & 1 & I & & & $126^{5}$ & I \\
\hline$-0,006$ & & 1514 & 13 & 1 & 1 & & & $z=$ & $T$ \\
\hline-0006 & & 1515 & 14 & I & $I$ & & & 125 & $\downarrow$ \\
\hline-0007 & & 1520 & 15 & & $T$ & & & 23 & \\
\hline$. \quad-0007$ & & 1521 & 16 & & i & & & 125 & \\
\hline-0008 & & 1528 & 12 & & 1 & & & 22 & \\
\hline-0008 & & 1,25 & E & 1 & $!$ & & & 125 & \\
\hline-0009 & & 1533 & 19 & 1 & t & & & 22 & 巫 \\
\hline-6009 & & $153 \mathrm{~N}$ & 20 & I & t & & & 125 & MAvíz \\
\hline-0010 & & $1 \sqrt{29}$ & 21 & $T$ & & & & 22 & 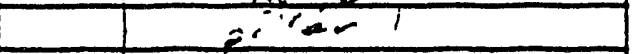 \\
\hline$-\infty 810$ & & 15240 & 22 & 1 & 1 & & & 125 & $+12 h \div A i !=U A$ \\
\hline-00104 & & 1544 & 23 & $i$ & & & & 22 & 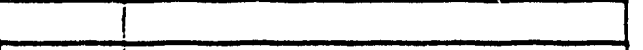 \\
\hline-100104 & & 1545 & 24 & $!$ & 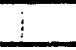 & & & 125 & \\
\hline$-\tilde{x} \dot{x} \dot{\rho} \mid k L$ & & 1847 & 25 & I & $!$ & & & 22 & I \\
\hline$-3 y+41 k 2$ & & 1548 & 26 & $I$ & 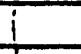 & & & 125 & \\
\hline-5011 & & 1552 & 27 & $I$ & 5 & & & 22 & $T$ \\
\hline$-00: 1$ & & 1583 & 23 & $i$ & $=$ & & & 125 & T \\
\hline-0012 & & 1559 & 24 & 1 & 6 & & & 22 & 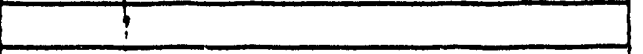 \\
\hline-0012 & & 1600 & 30 & 1 & 1 & & & 125 & I \\
\hline$-00 / 3$ & & 1603 & $3 i$ & $!$ & 1 & & & 22 & I \\
\hline-0013 & & 1604 & 32 & $i$ & 1 & & & 125 & \\
\hline-0014 & & 1609 & 33 & 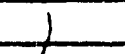 & $i$ & & & 22 & \\
\hline-0014 & & 1610 & 34 & 1 & $I$ & & & 125 & t \\
\hline$-00 / 5$ & $11 / 5$ & 0929 & 35 & 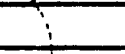 & & & & 22 & \\
\hline-0015 & $11 / 5$ & 2740 & Is & $T$ & $!$ & & & 125 & \\
\hline-2016 & & 0743 & 37 & $i$ & & & & 22 & \\
\hline$-001 \%$ & 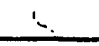 & 1744 & 38 & 1 & 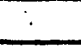 & & & 125 & \\
\hline$-100,7$ & 1 & 0749 & 39 & $I$ & & & & 22 & \\
\hline$-\infty 017$ & $i$ & 0750 & 40 & $I$ & $i$ & & & 125 & \\
\hline-0018 & $I$ & Dnन्य & 41 & $I$ & $I$ & & & 22 & \\
\hline$\rightarrow 0,9$ & 1 & 0755 & 42 & & $T$ & & & $12:$ & \\
\hline-0019 & & 0759 & 43 & & & & & & \\
\hline
\end{tabular}


NICROSEEPS LTO.

aIEn: WSRc

Location: $240-[$

PROJECT(1): TASL!

PAGE: $z$ OF:

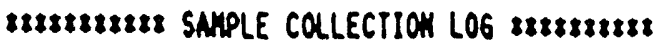

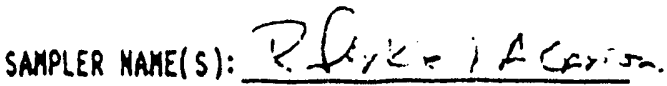

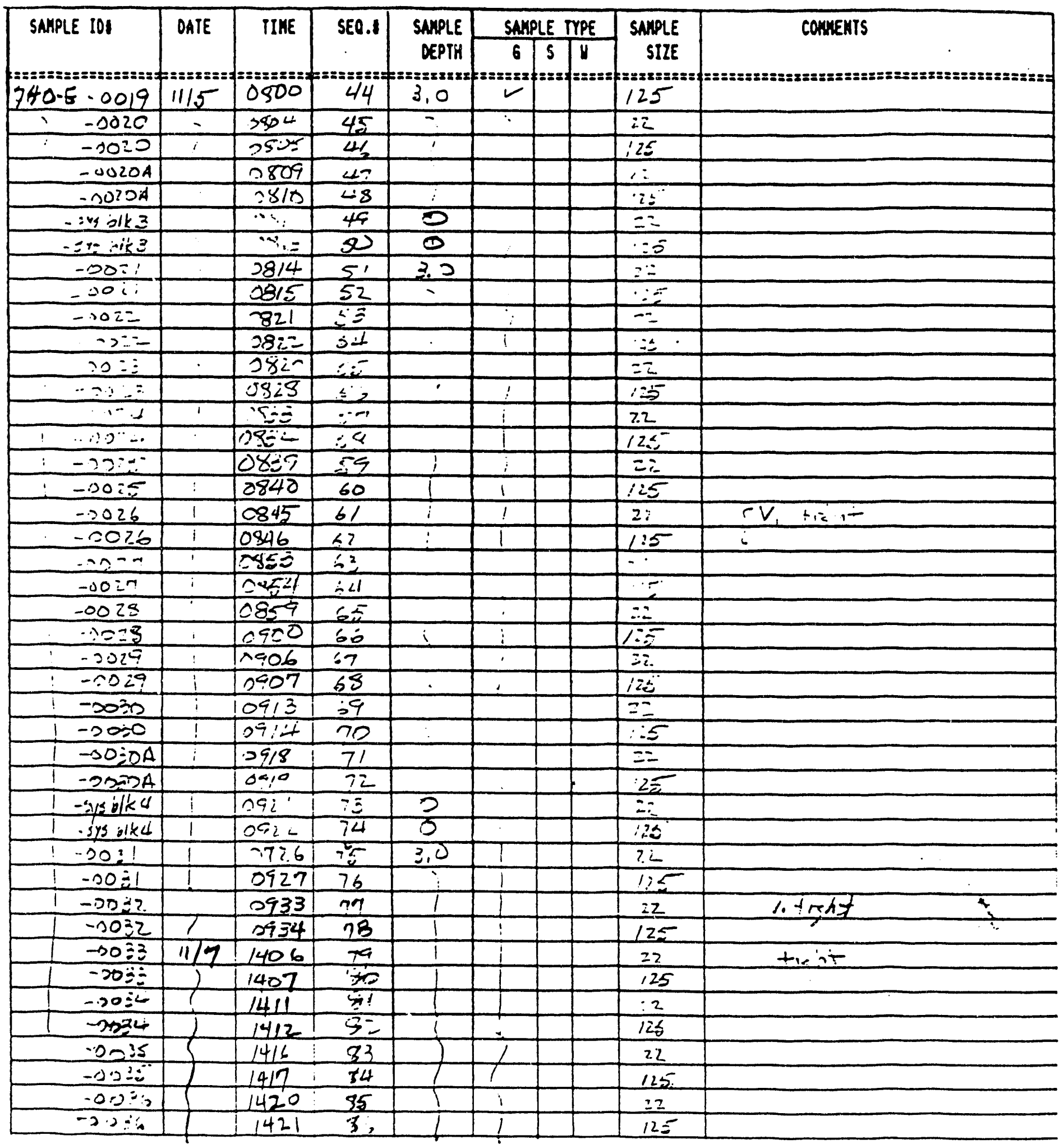


MICROSEEPS LTO.

aIm: WJRC

Locilion: $740-E$

PROSECI(1): TASEI

PAGE: 3 Of:

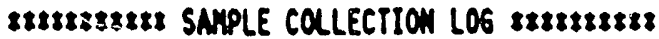

SAMPLER MAME(S):

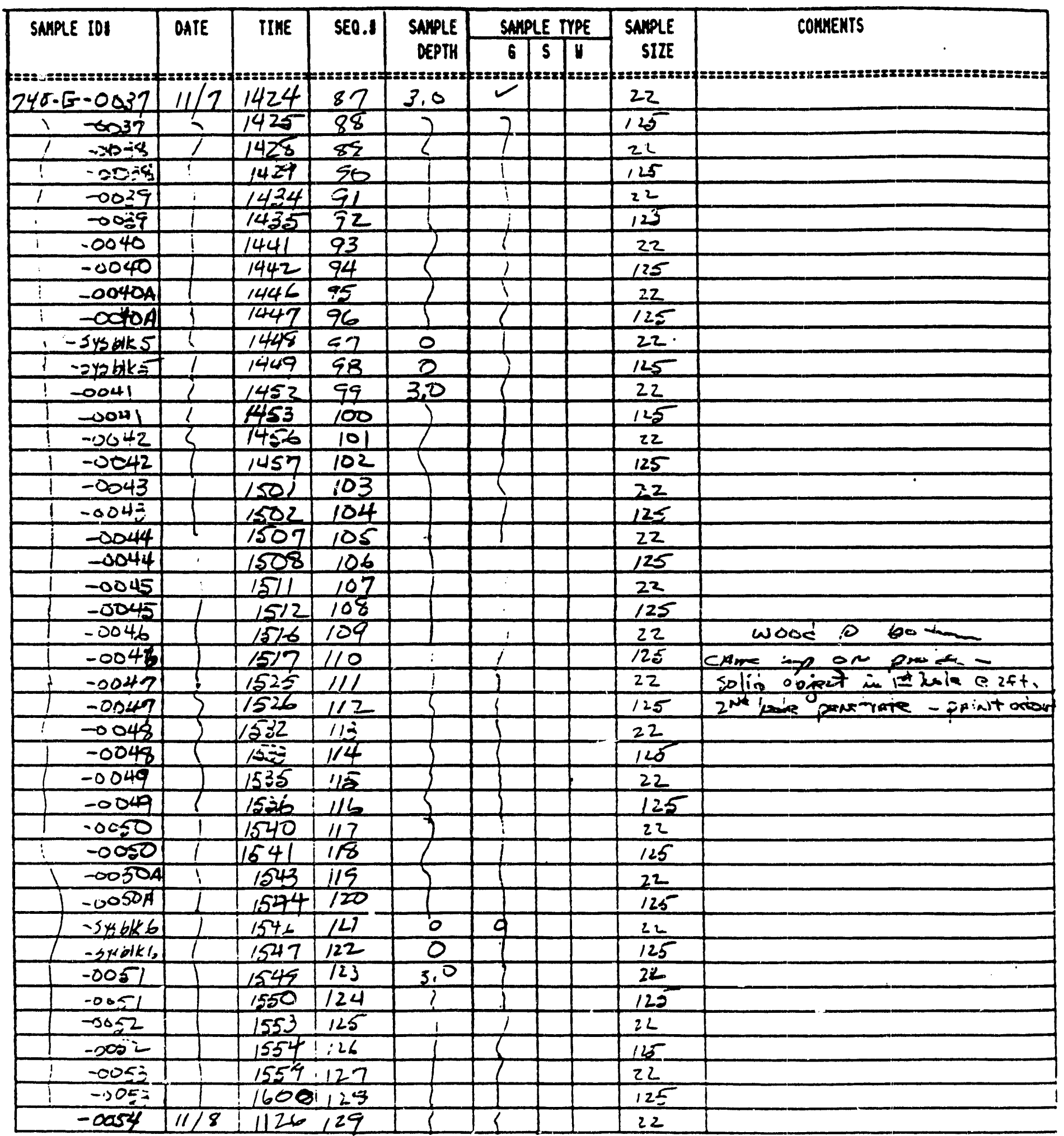


MICROSEEPS LTD.

aIET: $\quad$ WSRC

Lochirom: $\quad 740-E$

PROJECT(1): TRKKI

PHeE: 4 of:

82888882888 SAPLE COLLCTION LOS 8288825828

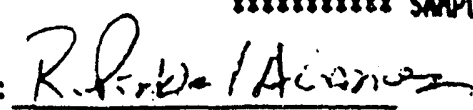

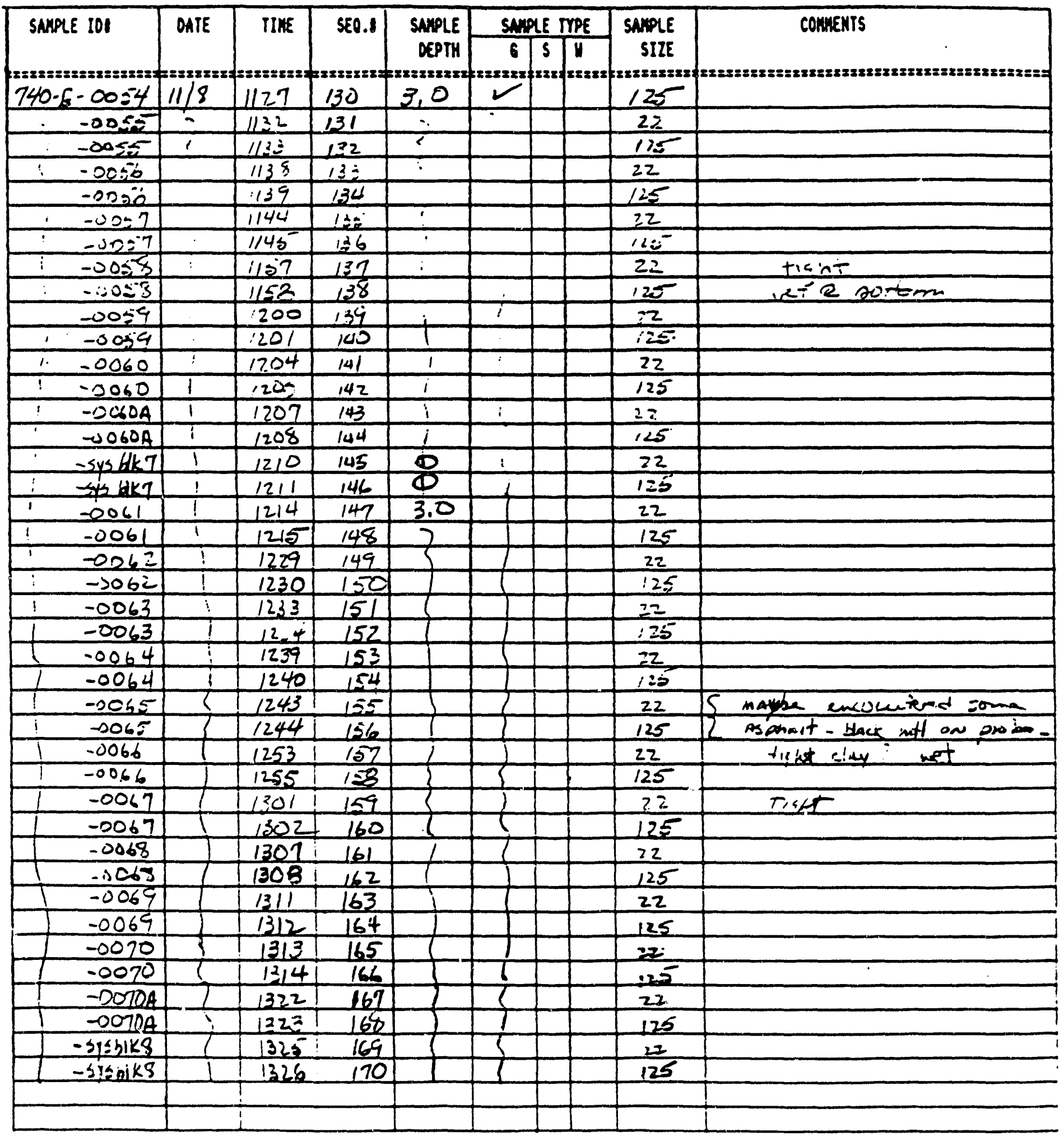


MICROSEEPS LID.

QIEn: $\quad-W S R C$

LochilON: $G$ LANDFILL

PROJECT(E): TAYR 1A

PACE: $\perp$ of:

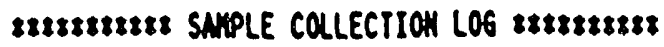

SAMPLER MaME(S):RJP DUM AGC

\begin{tabular}{|c|c|c|c|c|c|c|c|c|c|}
\hline SAMPLE IOI & DATE & TIME & SEO.1 & SAMPLE & & PLE & TYPE & SAMPLE & COAnENTS \\
\hline 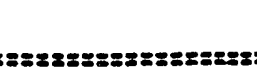 & s:z=xz== & & $23232=28$ & $\begin{aligned} \text { DEPTH } \\
z x z z \leq z 2 x z\end{aligned}$ & 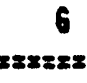 & 5 & $\prod_{2 x=2 x}^{1}$ & $\begin{array}{c}\text { SIZE } \\
: x=2 x z 2 z x=\end{array}$ & 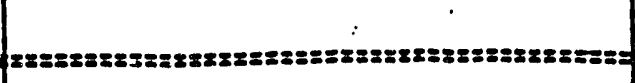 \\
\hline Sys BlK 9 & $11-9$ & & 1 & $3.0^{\circ}$ & 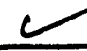 & & & 22 & \\
\hline Sus BIK 9 & & & 2 & $r$ & & & & 125 & \\
\hline$G-2$ & 7 & & 3 & & 7 & & & 32 & \\
\hline $6-31$ & $T$ & & 4 & & 1 & & & 125 & \\
\hline$a-22$ & $T$ & & 5 & & $i$ & & & 22 & \\
\hline$G-32$ & $T$ & & 6 & & & & & 125 & NO IIRESS. \\
\hline 6.23 & 1 & & 2 & & $i$ & & & 22 & \\
\hline$c-23$ & $i$ & & 8 & & 1 & & & 125 & No dices wijo- \\
\hline$G-32 A$ & $\vdots$ & $11: 30$ & 9 & & 1 & & & 125 & Leramp!e's cute \\
\hline$G-22 A$ & $\vdots$ & $11: 35$ & 10 & & $i$ & & & 125 & Ressmples :i-e \\
\hline $6-74$ & 1 & $11: 37$ & 11 & & $T$ & & & 22 & \\
\hline$G-24$ & $i$ & $11: 38$ & 12 & & 1 & & & $1=5$ & \\
\hline 6.75 & $\zeta$ & $11: 41$ & 13 & & 1 & & & 22 & \\
\hline$G-25$ & & $11: 42$ & 14 & & $!$ & & & 125 & \\
\hline $6-76$ & & 11.47 & $15^{\circ}$ & $\vdots$ & $\frac{1}{1}$ & & & 32 & \\
\hline $6-.76$ & & $11: 45$ & 16 & $i$ & ! & & & 125 & \\
\hline$G-79$ & & $11: 50$ & 17 & $T$ & $i$ & & & 22 & \\
\hline$G-19$ & & $11: 51$ & 18 & $\dot{\Sigma}$ & $i$ & & & 125 & \\
\hline Sys BIK 10 & & $11: 55$ & 19 & & $i$ & & & 72 & \\
\hline $\operatorname{sus} \beta / h 10$ & $i$ & $11: 56$ & 20 & & $i$ & & & 125 & \\
\hline $3-12$ & & $12: 01$ & 21 & & $i$ & & & .2 & \\
\hline 6.9$)^{\circ}$ & $:$ & $12: 02$ & 22 & & 1 & & & 125 & \\
\hline $6-78$ & ! & $12: 04$ & 83 & & $\therefore$ & & & 22 & \\
\hline $6-28$ & & $12: 05$ & 24 & & $i$ & & & 125 & \\
\hline 6.80 & & $12: 08$ & 25 & & & & & 22 & \\
\hline$G-80$ & & $12: 19$ & 26 & $\therefore$ & & & & 175 & \\
\hline G-80A & & $12: 11$ & 29 & & & & & 22 & \\
\hline 9.904 & & $12: 12$ & 28 & & & & & 125 & \\
\hline 5.81 & & $12: 19$ & 29 & & & & & 22 & \\
\hline$G-81$ & & $12: 15$ & 30 & $i$ & & & & 125 & \\
\hline 6.82 & & $12: 25$ & 31 & 1 & $\vdots$ & & & 22 & \\
\hline $6-82$ & & $12: 26$ & 32 & $j$ & $!$ & & & 125 & \\
\hline $6-83$ & $\vdots$ & 12.30 & 33 & 7 & 1 & & & $1=$ & \\
\hline $6-83$ & $i$ & $12: 31$ & 34 & $\tau$ & $\div$ & & & $1=\div$ & \\
\hline$=-9.4$ & $!$ & $12: 34$ & 35 & & & & & 32 & \\
\hline$G-34$ & $i$ & $: 2: 35$ & 36 & & & & & 125 & \\
\hline$G-85$ & $:$ & $12: 37$ & 37 & & 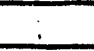 & & & 22 & \\
\hline $15-85$ & & $12 \cdot 38$ & 35 & & $!$ & & & $: .5$ & \\
\hline $6-86$ & & $13: 10$ & 39 & & 1 & & & 22 & \\
\hline$G-86$ & 1 & $12: 41$ & 40 & & $T$ & & & 125 & \\
\hline $5 \leq 7$ & & $14: 35$ & 41 & 1 & 1 & & & 22 & \\
\hline G 87 & $i$ & $14: 36:$ & 42 & $i$ & $i$ & & & 120 & \\
\hline$E S$ & $t$ & & 45 & T & $i$ & & & 22 & \\
\hline
\end{tabular}


aran: WSRC

cocurion: $740-E$

PROJECT(1): TPJK

PAGE: 2 OF:

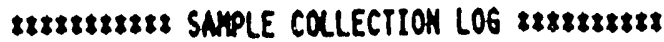

SaApler mane(s): Grele / Carron

\begin{tabular}{|c|c|c|c|c|c|c|c|c|c|}
\hline SAMPLE IOI & OATE & IIME & SEO.1 & SMPPLE & & LET & & SAMPLE & COWHENTS \\
\hline 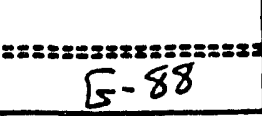 & & & & $\begin{array}{l}\text { DEPTH } \\
\text { :z:=:zz:x: }\end{array}$ & 6 & s & $y$ & 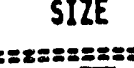 & 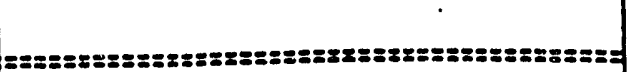 \\
\hline$\frac{G-88}{G-89}$ & $11 / 9$ & 1531 & 44 & 3.0 & $c$ & & & 125 & \\
\hline$\frac{G-89}{\sqrt{5}-89}$ & 7 & 1534 & 45 & & 5 & & & $2:$ & \\
\hline$\frac{\sqrt{5}-89}{\sqrt{6}-0.0}$ & & 1535 & 46 & ) & I & & & 125 & \\
\hline$\frac{E^{-0.0}}{E-00}$ & I & 1539 & 47 & 1 & $\frac{1}{1}$ & & & 22 & \\
\hline$\frac{E-\infty}{E-90 A}$ & $T$ & 1540 & 48 & $!$ & 5 & & & 125 & \\
\hline$\frac{\sqrt{5}-90 \mathrm{~A}}{E-90 \mathrm{~A}}$ & 1 & $1=22$ & 49 & 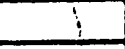 & $i$ & & & 2.2 & \\
\hline$\frac{E-90 A}{E-2 \times s / k d 1}$ & $i$ & 1544 & 50 & 1 & 1 & & & $125^{-}$ & \\
\hline 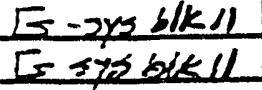 & I & 1546 & 51 & 1 & $\vdots$ & & & $\because$ & \\
\hline$\frac{[5=575 \text { bikll }}{\mid r-51}$ & 1 & 1547 & 52 & I & $i$ & & & $120^{\circ}$ & \\
\hline$\frac{\mid r-91}{E-71}$ & 1 & 1545 & 52 & $i$ & $i$ & & & $\therefore=$ & \\
\hline$\frac{5-51}{5-72}$ & 1 & 1550 & 54 & $i$ & $i$ & & & $\therefore$ & \\
\hline$\frac{5-12}{5-52}$ & $\frac{1}{1}$ & $\frac{1553}{15-4}$ & 55 & $i$ & $\therefore$ & & & $\therefore=$ & \\
\hline$E-\sqrt{3}$ & $\frac{1}{1}$ & $\frac{1554}{1557}$ & 52 & $\therefore$ & 1 & & & 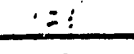 & \\
\hline$L_{S}-s=$ & $i$ & $\frac{1637}{15 x 5}$ & $\frac{57}{58}$ & 1 & $i$ & & & $\therefore=$ & \\
\hline$E-94$ & $i$ & 1002 & $\frac{38}{59}$ & $\frac{1}{1}$ & 3 & & & $\therefore$ & \\
\hline$E-94$ & & 160.3 & 60 & $i$ & $i$ & & & $\because \cdots$ & \\
\hline $6-95$ & & 1007 & 61 & $i$ & $!$ & & & $\therefore$ & \\
\hline 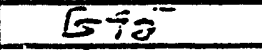 & & 1600 & 62 & 7 & & & & $\therefore \therefore$ & \\
\hline 1576 & 1 & 16613 & 63 & $i$ & & & & $i=$ & \\
\hline 596 & & 1614 & 64 & $T$ & 5 & & & $\therefore$ & \\
\hline$E=?$ & 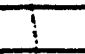 & $16 / 8$ & 65 & I & 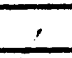 & & & 2.2 & \\
\hline$E \rightarrow ?$ & $i$ & 16,13 & 66 & 1 & $\vdots$ & & & $\therefore$ & \\
\hline$E 78$ & $T$ & 1623 & 67 & $i$ & ! & - & & $\because$ & \\
\hline$E>5$ & & 1624 & 63 & & 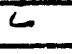 & & & $1 \%$ & \\
\hline & & & & & & & & & \\
\hline & & & & & & & & & \\
\hline & & & & & & & & & \\
\hline & & & & & & & & & \\
\hline & & & & & & & & & \\
\hline & & & & & & & & & \\
\hline & & & & & & & & & \\
\hline & & & & & & & & & \\
\hline & & & & & & & & & \\
\hline & & & & & & & & & \\
\hline & & & & & & & & & \\
\hline & & & & & & & & & \\
\hline & & & & & & & & & \\
\hline & & & & & & & & & \\
\hline & & & & & & & & & \\
\hline
\end{tabular}


aIEn: $\quad \mu s R C$

Lochirion: $240-5$

PROJECT(8): TASKI

PAGE: 1 of:

sampler mame(s): K. Pirkle/e.MosLat

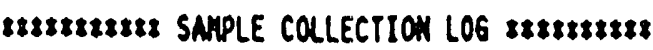

surer inetsl: Ki Hirkle/elmoshat

\begin{tabular}{|c|c|c|c|c|c|c|c|c|c|}
\hline \multirow{2}{*}{ SARPLE IOI } & \multirow{2}{*}{ OATE } & \multirow[t]{2}{*}{ TIME } & \multirow[t]{2}{*}{ SEO.I } & \multirow{2}{*}{$\begin{array}{l}\text { SANPLE } \\
\text { DEPTH }\end{array}$} & \multicolumn{3}{|c|}{ SAYPLE IYPE } & \multirow{2}{*}{$\begin{array}{r}\text { SAMPLE } \\
\text { SIZE }\end{array}$} & \multirow[t]{2}{*}{ COHAENTS } \\
\hline & & & & & 6 & 5 & 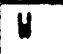 & & \\
\hline & (1) & & & & & & & & 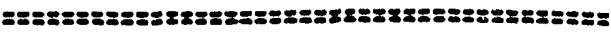 \\
\hline$\frac{5-49}{5-55}$ & 11/11 & 1121 & 1 & 3,0 & 2 & & & 22 & \\
\hline$\frac{G-95}{5-100}$ & 1 & 1122 & 2 & $>$ & 1 & & & 225 & \\
\hline$\frac{G-100}{G-100}$ & 1 & 1127 & 3 & 1 & $\therefore$ & & & $2 z$ & \\
\hline$\frac{G-100}{G-100 A}$ & 1 & 1128 & 4 & & $\therefore$ & & & 125 & \\
\hline$\frac{b-100 A}{G-100 A}$ & & $\mu=0$ & $s$ & $\therefore$ & 1 & & & $z=$ & \\
\hline$G-100 A$ & $i$ & 1131 & 6 & & $\vdots$ & & & 125 & \\
\hline$G-31 s d k 12$ & 1 & 1133 & 7 & $\mathbf{0}$ & 1 & & & 22 & \\
\hline E-siss dedk 12 & 1 & 113L & 8 & 0 & $\vdots$ & & & 125 & \\
\hline$G-101$ & & 1138 & 9 & 30 & 1 & & & 22 & \\
\hline$=-101$ & 1 & $11 \div 9$ & 10 & & 1 & & & $12=$ & \\
\hline $5-10=$ & 1 & 1143 & 11 & I & & & & $z^{2}$ & \\
\hline $5-102$ & 1 & 1144 & 12 & 1 & 1 & & & 125 & \\
\hline$E \cdot 10 j$ & & 1149 & $\sqrt{3}$ & & $I$ & & & $z z$ & int \\
\hline$G \cdot 103 \mathrm{~A}$ & & 1150 & 14 & & I & & & $-2=22$ & \\
\hline$[-104$ & & 1156 & 5 & I & I & & & 22 & \\
\hline$[-7 \$ 04$ & & 1157 & 16 & 1 & I & & & 125 & \\
\hline $5-105$ & & 1204 & 17 & I & I & & & 22 & \\
\hline $5-105$ & & 1205 & 18 & & $E$ & & & 125 & \\
\hline $5=106$ & & 1210 & 19 & 1 & 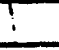 & & & 22 & \\
\hline $5+106$ & & 1211 & 20 & & $i$ & & & 125 & \\
\hline$[2-10]$ & & 1215 & 21 & & $!$ & & & 22 & \\
\hline$[--107$ & & 1216 & 22 & & 1 & & & 125 & \\
\hline $5-108$ & & $12=9$ & 23 & & 1 & & & 22 & \\
\hline $5-108$ & & 1221 & 24 & & 1 & & & 125 & \\
\hline$\sqrt{5}-109$ & & 1225 & 25 & & I & & & 22 & \\
\hline$E-10 G$ & & 1220 & 26 & & & & & 125 & \\
\hline$E-110$ & & 1235 & 27 & & & & & 22 & \\
\hline$[-110$ & & 1234 & 28 & & & & & 125 & \\
\hline$S_{5}-110 \mathrm{~A}$ & & 1236 & 25 & & & & & $m$ & \\
\hline$r_{2}-110 A$ & & 1237 & 20 & & & & & 125 & \\
\hline E. sys blk 13 & & 1238 & 31 & $\phi$ & & & & $2-$ & \\
\hline$[x-9 y<\operatorname{ink13}$ & & 1237 & 32 & $\Phi$ & $L$ & & & 125 & \\
\hline$[E-111$ & & 1244 & 33 & $3 \sqrt{0}$ & & & & 22 & \\
\hline$G-111$ & & 1245 & 34 & 7 & $\perp$ & & & 125 & \\
\hline$[-112$ & & 1247 & 35 & & & & & 22 & \\
\hline$\frac{5}{5}-112$ & & 1248 & 36 & & & & & 125 & \\
\hline$E-1 / 6$ & & 1441 & 37 & 1 & & & & 23 & SAmekd then dumped soll \\
\hline$G-116$ & & 1442 & 38 & 1 & & & & 125 & \\
\hline$G-117$ & & 1446 & 39 & & & & & 22 & \\
\hline $5-117$ & & 1447 & 40 & & & & & 125 & \\
\hline $5-118$ & & 1453 & 41 & 1 & & & & 22 & \\
\hline$E-119$ & & 1454 & 42 & I & & & & 125 & \\
\hline $5-121$ & & 1503 & 42 & 1 & & & & $i 2$ & $\downarrow$ \\
\hline
\end{tabular}


MICROSEEPS LTO.

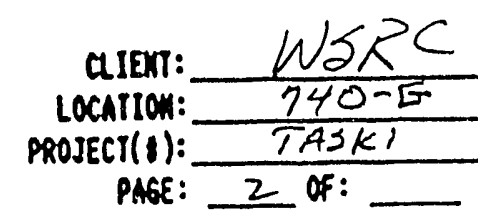

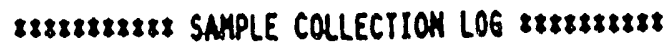

sampler hane(s): RPiskle. D. Masoda

\begin{tabular}{|c|c|c|c|c|c|c|c|c|c|}
\hline \multirow{2}{*}{ 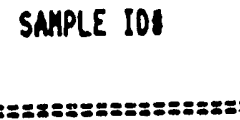 } & \multirow[t]{2}{*}{ OATE } & \multirow[t]{2}{*}{ TIME } & \multirow[t]{2}{*}{ SEO.1 } & \multirow{2}{*}{$\begin{array}{l}\text { SAHPLE } \\
\text { DEPTH }\end{array}$} & \multicolumn{3}{|c|}{ SAMPLE TYPE } & \multirow{2}{*}{$\begin{array}{l}\text { SAMPLE } \\
\text { SIZE }\end{array}$} & \multirow{2}{*}{ 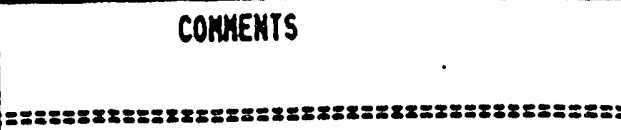 } \\
\hline & & & & & 6 & s & y & & \\
\hline$E-121$ & $11 / 11$ & 1504 & 44 & 3.0 & $\checkmark$ & & & 125 & ba toil ites \\
\hline$E-122$ & $11 / 11$ & 1208 & 45 & 1 & - & & & 22 & 11 \\
\hline $5-122$ & & 1504 & 46 & I & 1 & & & 115 & \\
\hline$G-123$ & $i$ & 1.515 & 47 & 1 & 1 & & & 22 & \\
\hline $5-123$ & & 1516 & 48 & & & & & 125 & \\
\hline$\frac{5 \cdot 130}{2}$ & $!$ & 1524 & 19 & 1 & 1 & & & $=2$ & \\
\hline$\frac{\sqrt{5}-150}{\frac{5}{5}-131}$ & & 15,5 & 50 & $T$ & 1 & & & 125 & \\
\hline$\frac{G-131}{G-121}$ & 1 & 1534 & 51 & I & 1 & & & $27=$ & \\
\hline$\frac{G-121}{G-121 A}$ & 1 & $18 \div 8$ & 52 & $i$ & $i$ & & & $125^{\circ}$ & \\
\hline$\frac{\sqrt{5}-131 A}{\sqrt{5}-131 A}$ & & 1545 & 52 & 1 & I & & & $2=$ & \\
\hline$\frac{\Gamma \cdot 131 A}{r_{-}-5 y^{4} \text { aik } 14}$ & I & 1546 & 54 & $I$ & $?$ & & & 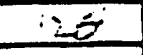 & \\
\hline$\frac{r_{-}-s y \leq a i k \mid 4}{G \cdot s y-i k: 4}$ & 1 & 1548 & 55 & $\Phi$ & 1 & & & $\because=$ & \\
\hline$\frac{G \cdot 3 y+2 / K: L}{E-132}$ & 1 & $15=19$ & 56 & 0 & 1 & & & 25 & \\
\hline$\frac{5-132}{5-127}$ & I & 1553 & 57 & 3.0 & $i$ & & & 22 & \\
\hline$\frac{5-127}{5-113}$ & 1 & 1554 & 53 & & 1 & & & 125 & $\underline{v}$ \\
\hline$\frac{\sqrt{5}-113}{5-113}$ & & 1606 & 59 & 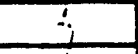 & & & & 22 & \\
\hline$\frac{5-113}{5-114}$ & & 1607 & 60 & 1 & $:$ & & & 125 & \\
\hline$\frac{G-114}{G-110}$ & 1 & 1610 & 61 & 1 & $!$ & & & 22 & \\
\hline$\frac{E-110}{E-115}$ & 1 & 1611 & 62 & 1 & 1 & & & 165 & \\
\hline$\frac{5-115}{5-115}$ & 1 & 1616 & 107 & $i$ & 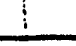 & & & 22 & \\
\hline & 1 & 1617 & 64 & $!$ & 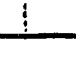 & & & 125 & \\
\hline & & & & & & & & & \\
\hline & & & & & & & & & \\
\hline & & & & & & & & & \\
\hline & & & & & & & & & \\
\hline & & & & & & & & & \\
\hline & & & & & & & & & \\
\hline & & & & & & & & & \\
\hline & & & & & & & & & \\
\hline & & & & & & & & & \\
\hline & & & & & & & & & \\
\hline & & & & & & & & & \\
\hline & & & & & & & & & \\
\hline & & & & & & & & & \\
\hline & & & & & & & & & \\
\hline & & & & & & & & & \\
\hline & & & $!$ & & & & & & \\
\hline & & & & & & & & & \\
\hline & & & & & & & & & 1 \\
\hline
\end{tabular}


MICROSEEPS LTO.

aram: WSRC

Locarion: $7 \%$ -

PROJECT(1): TASKI

PAGE: 1 of:

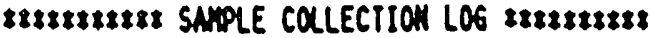 \\ sampler mane(s): P. firke d D.mos do}

\begin{tabular}{|c|c|c|c|c|c|c|c|c|c|}
\hline SAMPLE IOI & DATE & IIME & SEO.I & SAMPLE & & LE T & & SAPPLE & COMUENTS \\
\hline & & & & DEPTH & 6 & 5 & $y$ & SILE & \\
\hline$E-$ sps blk 15 & $11 / 12$ & $\begin{array}{l}32 x=25 x \\
1345\end{array}$ & $== \pm 3$ & 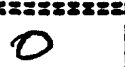 & $=2$ & & & 22 & 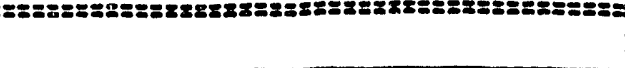 \\
\hline E-spos bik15 & 2 & isth & Z & 0 & 7 & & & 125 & \\
\hline$E-119$ & 7 & 353 & 3 & 2,0 & 1 & & & $i 2$ & \\
\hline$E-119$ & 1 & 1354 & 4 & 2 & $I$ & & & 11.5 & \\
\hline$E_{5}-120$ & 1 & 1354 & 5 & 1 & & & & 22 & $10^{\prime} \quad-w$ wond in - X \\
\hline$E-120$ & $!$ & 1400 & 5 & $\therefore$ & 1 & & & 125 & \\
\hline$\Gamma_{5}-123$ & - & 1403 & 7 & 1 & & & & 22 & 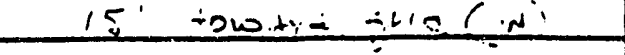 \\
\hline $5 \leq-123$ & 1 & 1404 & 3 & 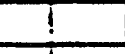 & $i$ & & & 125 & \\
\hline$E-124$ & 1 & 1467 & 9 & 1 & 1 & & & 22 & $h \sim a-=m a$ \\
\hline $5-1=4$ & I & 1408 & 10 & I & 1 & & & 125 & \\
\hline-135 & & 1411 & 11 & 1 & $I$ & & & 22 & tiajt \\
\hline$G-135$ & & $14 \mathrm{~L}$ & 12 & 1 & $I$ & & & 125 & \\
\hline$[0-136$ & & 1414 & 12 & 1 & I & & & $2 x$ & \\
\hline $5-136$ & & 1415 & 14 & 1 & 1 & & & 125 & \\
\hline $5-137$ & & 1420 & 5 & & 1 & & & $2-2$ & \\
\hline$F-137$ & & 1421 & 16 & $i$ & & & & 125 & \\
\hline $5-138$ & & 1425 & 17 & 1 & & & & 22 & \\
\hline$\sqrt{5}-138$ & & 1426 & 18 & I & & & & 125 & \\
\hline$E-139$ & & 1429 & 19 & & & & & 22 & \\
\hline $5-13$ & & 1430 & 20 & & 1 & & & 125 & \\
\hline $5-140$ & & 1436 & 21 & 1 & $i$ & & & 22 & \\
\hline$E-140$ & & 1437 & 22 & & $I$ & & & 125 & \\
\hline $5^{-140 A}$ & & 1439 & 23 & 1 & 1 & & & 22 & \\
\hline$E-140 A$ & 7 & 1440 & 24 & & & & & 125 & \\
\hline$G$ - sot belk 16 & & 1443 & 25 & & & & & $\div 2$ & \\
\hline$[2-3 y s$ h)k16 & 1 & 1444 & 26 & & & & & 125 & \\
\hline $5-127$ & & 1447 & 27 & 1 & & & & 22 & \\
\hline$G-127$ & & 1448 & 28 & & & & & 125 & \\
\hline$E-128$ & & 1453 & 27 & & & - & & 22 & \\
\hline$E-128$ & & 1454 & 30 & & & & & 125 & \\
\hline$G-129$ & & 1455 & 31 & & & & & 22 & \\
\hline$G-129$ & & 1456 & 32 & & & & & 125 & \\
\hline$G-125 A$ & i & 1457 & 33 & & & & & 22 & \\
\hline$G-129 A$ & I & 1500 & 34 & & & & & 125 & \\
\hline $5 \cdot 124$ & 1 & 1503 & 35 & & & & & 22 & \\
\hline $5-124$ & 1 & 1504 & 36 & & & & & 125 & \\
\hline$I_{I}-125$ & 1 & 1507 & 37 & & & & & 22 & \\
\hline$E-125$ & & 1508 & 23 & & & & & 126 & \\
\hline$E-126$ & & 1512 & 35 & & & & & $2 \ldots$ & \\
\hline$G-12 i$ & & 1512 & 40 & & & & & 126 & \\
\hline & & & & & & & & & \\
\hline & & i & & & & & & & \\
\hline & & & & & & & & & \\
\hline
\end{tabular}


MICROSEEPS LID.

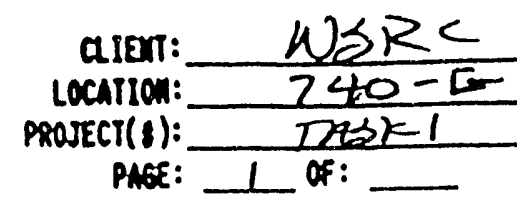

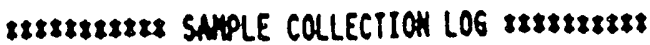

Sampler hame(s): ROPI DOMM

\begin{tabular}{|c|c|c|c|c|c|c|c|c|}
\hline SAMPLE IOI & DATE & TYME & SEO.1 & SAPLE & & TYPE & SAMPLE & COHnENTS \\
\hline & & & & 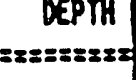 & 6 & $\int_{2=:=2}$ & SILE & 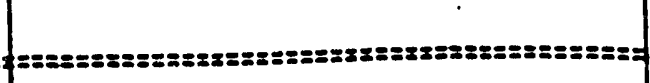 \\
\hline $5-156$ & $11 / 13$ & 1622 & 1 & 3,0 & $\checkmark$ & & 22 & \\
\hline$G-156$ & 7 & 1623 & 2 & 1 & $L$ & & 125 & \\
\hline $5-157$ & $!$ & 1624 & 3 & 1 & T & & 22 & \\
\hline $5-157$ & 1 & 1625 & 4 & & 1 & & .1 .5 & \\
\hline $5-13$ & 1 & 1626 & 3 & & 1 & & 22 & \\
\hline$\sqrt{5-123}$ & $i$ & 1627 & $\therefore$ & 1 & 1 & & 1.5 & \\
\hline$E-15 Y$ & 1 & 1630 & $?$ & 1 & $!$ & & $\div 2$ & \\
\hline$E-157$ & 1 & 1631 & $j$ & 1 & 1 & & 135 & \\
\hline$E-160$ & 1 & 1635 & $\dot{i}$ & I & 1 & & 22 & \\
\hline $5-160$ & 1 & 1636 & 10 & 1 & 1 & & .25 & \\
\hline$F-50 A$ & $T$ & 1637 & $\therefore$ & 1 & 1 & & 12 & \\
\hline$\sqrt{5}-100 \Delta$ & $T$ & 1638 & 12 & 1 & $I$ & & 125 & \\
\hline$G-5 y \div d k / 7$ & 1 & 1638 & $\therefore 2$ & 0 & 1 & & 22 & \\
\hline$\left[E-3 \gamma^{3} \mathrm{~d} / \mathrm{k} / 7\right.$ & 1 & 1639 & 14 & 0 & 1 & & 123 & \\
\hline$[5-|6|$. & 1 & 1641 & 13 & 3.0 & 1 & & \pm 2 & \\
\hline$E=161$ & 7 & 1642 & 16 & 1 & 1 & & .125 & \\
\hline$z-168$ & $11 / 12$ & 1335 & 17 & 2 & 1 & & 22 & \\
\hline$G-168$ & 5 & 1336 & 18 & I & $i$ & & 185 & \\
\hline$G-141$ & I & 1346 & 19 & 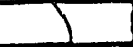 & 1 & & 22 & \\
\hline $5-141$ & $i$ & 1347 & 20 & & & & 12,5 & \\
\hline$[-16]$ & 1 & 1351 & 21 & & 1 & & 2.2 & \\
\hline $5-167$ & 1 & 1352 & 22 & & & & 125 & \\
\hline$E-142$ & 1 & 1354 & 23 & & & & 22 & \\
\hline $5-142$ & 1 & 135.5 & 24 & & & & 125 & \\
\hline $5-166$ & $\therefore$ & 1356 & 25 & 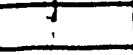 & & & 22. & \\
\hline$G-166$ & $\therefore$ & 1357 & 26 & 1 & & & 125 & \\
\hline$G-143$ & . & 1400 & 27 & 1 & & & 22 & \\
\hline$E-142$ & 1 & 1401 & 28 & 1 & & & 125 & \\
\hline $5-165$ & I & 1403 & 29 & 1 & & & $i 2$ & \\
\hline$E-165$ & 1 & 1404 & 30 & & & & 125 & \\
\hline $5-144$ & T & 1406 & 31 & $\perp$ & & & 22 & \\
\hline$\sqrt{5}-144$ & $T$ & 1407 & 32 & 1 & $\Delta$ & & 125 & \\
\hline $5-164$ & $!$ & 1410 & 33 & & 1 & & 22 & \\
\hline$G-164$ & $\bar{\vdots}$ & 1411 & 34 & & 1 & & 125 & \\
\hline $5-51231 k 18$ & $i$ & 1415 & 35 & 0 & & & $2=$ & \\
\hline C-syz bik 18 & 1 & 1414 & 36 & 0 & 1 & & 145 & \\
\hline$G=145$ & $i$ & $14 / 8$ & 37 & 3.0 & 7 & & 22 & \\
\hline$G-145$ & 1 & 1419 & 38 & & & & 125 & \\
\hline$G-163$ & 1 & 1424 & 39 & T & & & $2 ?$ & \\
\hline$r=163$ & 1 & 14251 & 40 & $T$ & & & 126 & \\
\hline$E-146$ & 1 & 1422 & 41 & $\vdots$ & & & 22 & \\
\hline$E-146$ & $T$ & 1429 & 42 & $i$ & & & $125^{-}$ & \\
\hline${ }_{5}-162$ & 1 & 14.54 & +3 & 1 & & & 22 & \\
\hline
\end{tabular}


MICROSEEPS LTO.

\section{Shypler hame(s): Kfofid DJm}

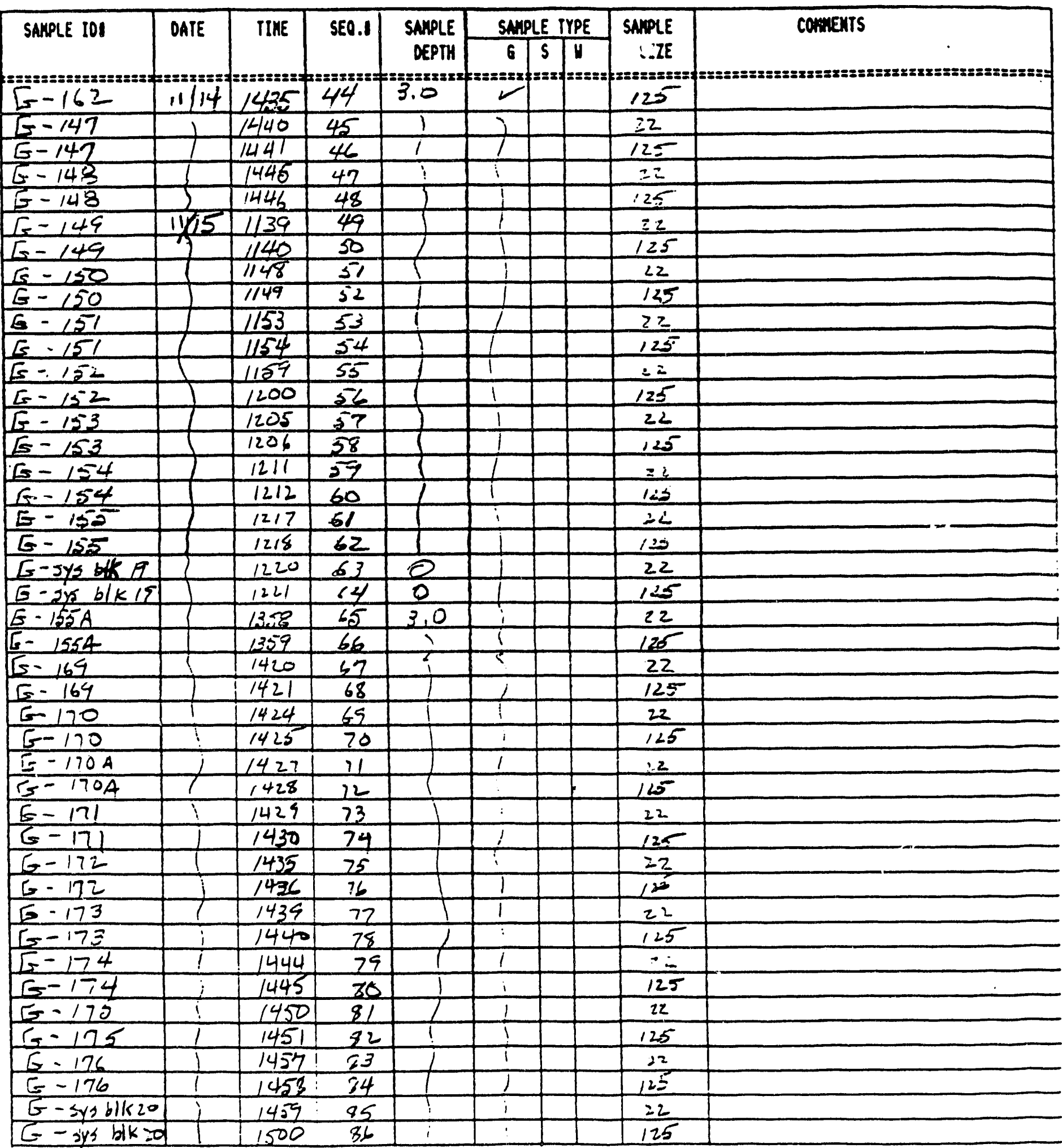


aIEN: $W S R C$

Lochirlon: 740-E

PROJECT(1): TASLI

PAGE: 3 OF:

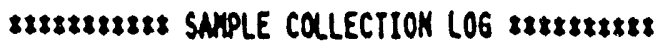

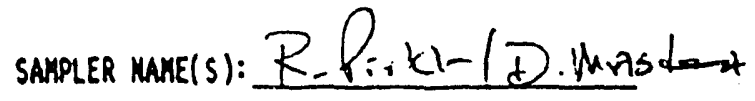

\begin{tabular}{|c|c|c|c|c|c|c|c|c|c|}
\hline SAMPLE IOS & OATE & TIME & SEQ.1 & SAMPLE & & LE & TYPE & SAMPLE & COMHEXTS \\
\hline & $=2=-20=0$ & & & OEPTH & 6 & $\mathbf{S}$ & $y$ & SIZE & \\
\hline$[s-127$ & $11 / 15$ & $\begin{array}{l}=2:=2828 \\
1505\end{array}$ & $\begin{array}{l}8=2 x=2 \\
87\end{array}$ & $\begin{array}{l}=2 x=28 x=x \\
3.0\end{array}$ & $\sqrt{28 z 28}$ & 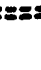 & $z=2=28$ & $\begin{array}{c}x=282=282 \\
22\end{array}$ & 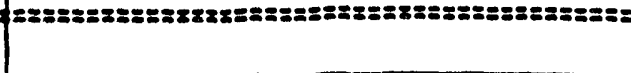 \\
\hline$[5-17]$ & $\because$ & 1506 & .53 & & 1 & & & 125 & \\
\hline$E \cdot 175$ & $:$ & 1510 & $8^{4}$ & & & & & $\because$ & \\
\hline$E-178$ & $T$ & 1511 & $\Xi 0$ & & . & & & $\equiv=$ & \\
\hline$G-179$ & 1 & 1515 & S1 & & & & & $2 i$ & \\
\hline$E-179$ & $i$ & 1576 & 72 & $!$ & T & & & $12=$ & \\
\hline$E-180$ & $T$ & 1520 & 53 & 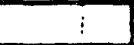 & $T$ & & & $\therefore-$ & \\
\hline $5-150$ & $!$ & 1521 & an & T & & & & $125^{-}$ & \\
\hline$E-180 A$ & 1 & 1525 & 95 & 1 & & & & 22 & \\
\hline$[-180 A$ & $\mathrm{i}$ & 1526 & 96 & $i$ & & & & 125 & \\
\hline$G-|S|$ & I & 1529 & 57 & 1 & & & & $\therefore=$ & \\
\hline$\Gamma_{\Sigma}-|<\Omega|$ & 1 & 1500 & $=8$ & 1 & & & & 125 & \\
\hline$E-5 y s$ blkzl & 1116 & 1349 & 95 & 0 & & & & 22 & \\
\hline$E-s y s$ błk 21 & $\mathrm{i}$ & 1356 & 100 & 0 & & & & 125 & \\
\hline $5-182$ & 1 & 1355 & 101 & $\exists \infty$ & & & & 22 & \\
\hline$G-182$ & & 1356 & 102 & & & & & 125 & \\
\hline$E-183$ & & 1359 & 103 & & & & & 22 & \\
\hline$E-183$ & & 1400 & 104 & & 1 & & & 125 & \\
\hline$G-184$ & & 1405 & 105 & & 1 & & & 22 & \\
\hline$E-184$ & I & 1406 & 106 & $S$ & & & & 126 & \\
\hline$E-145$ & 1 & 1410 & 107 & & & & & 22 & \\
\hline$\sqrt{5}-185$ & 1 & 1411 & 108 & & & & & 125 & \\
\hline$\sqrt{5}-186$ & I & 1414 & 109 & & & & & 2.2 & \\
\hline$E-186$ & & $14 / 5$ & 110 & 1 & 1 & & & 125 & \\
\hline$E-187$ & & 1419 & 111 & & & & & 22 & \\
\hline $5-187$ & & 1420 & 112 & & & & & 125 & \\
\hline $5-188$ & 1 & 1424 & 113 & 1 & & & & 22 & \\
\hline$=-135$ & 1 & 1425 & 114 & L & & & & 125 & \\
\hline$\sqrt{3}-149$ & & 1429 & 116 & 7 & & & & 22 & \\
\hline $5-145$ & 7 & 1430 & 116 & & 1 & & & 125 & \\
\hline$E \cdot 190^{\prime}$ & I & 1433 & 117 & $I$ & & & & 22 & \\
\hline$r-150$ & 1 & 1434 & $1 / 8$ & 1 & & & & 125 & \\
\hline$G-190 A$ & & 1437 & 119 & $i$ & & & & 22 & \\
\hline$G-1904$ & & 1438 & 120 & 1 & 1 & & & 125 & \\
\hline$E-s y s$ bikz & & 1440 & 121 & 0 & & & & $=2$ & \\
\hline$[2-5 \times 3, b \mid k 22$ & & 1441 & 122 & 0 & 1 & & & 125 & \\
\hline$G-191$ & I & 1444 & 123 & 3.0 & T & & & 22 & \\
\hline$G-191$ & 1 & 1445 & 124 & 1 & $T$ & & & 125 & \\
\hline$G=19 L$ & 1 & 1449 & $12=$ & & $i$ & & & $2 L$ & \\
\hline$G-152$ & $!$ & 1450 & 126 & L & $i$ & & & 125 & \\
\hline${ }_{c}-193$ & ) & 1474 & 127 & & $T$ & & & 22 & \\
\hline$r=-19 i$ & I & 1455 & 129 & & $I$ & & & $122^{\circ}$ & \\
\hline $5-194$ & T & 1453 & 129 & 1 & 1 & & & 22 & \\
\hline
\end{tabular}


arem: $\quad W S R L$

Lochirom: $740-E$

PROJECT(1): TRASK

PAGE: 4 of:

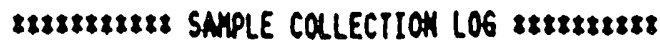

Sampler hane(s): R.P.ikle/D.tharisdon

\begin{tabular}{|c|c|c|c|c|c|c|c|c|c|}
\hline SMPLE IOI & DATE & TIME & SEQ.1 & SAMPLE & SAly & PLE & IYPE & SAMPLE & COHUENTS \\
\hline & & & & DEPTH & 6 & 5 & $\mathbf{4}$ & SIZE & \\
\hline$=$ & $\begin{array}{c}=2=28=2=: \\
11 / / 6\end{array}$ & $\begin{array}{l}==2=2=29 \\
1459\end{array}$ & $\begin{array}{c}32=28=2= \\
130\end{array}$ & $\begin{array}{c}: 2=2 x=2= \\
3,0\end{array}$ & =:2:28= & sez: & $==28=$ & $=2=28282=8$ & 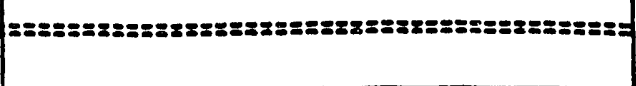 \\
\hline$G-195$ & $\therefore$ & 1004 & 131 & $?$ & 1 & & & $=2$ & \\
\hline$=-195$ & $\therefore$ & $1 \leq 2 \leq$ & 132 & i & 1 & & & 125 & \\
\hline $5 \cdot 15_{0}$ & 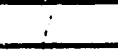 & 1511 & $1 \leq 3$ & $i$ & $!$ & & & 22 & \\
\hline 3.45 & $\vdots$ & 1512 & $\therefore \%$ & $\vdots$ & 1 & & & 125 & \\
\hline $5-197$ & ! & 1513 & 135 & 1 & - & & & 12 & \\
\hline$G-197$ & 4 & 1514 & 136 & L & $i$ & & & 125 & \\
\hline$G-198$ & $i$ & $15 i 9$ & 137 & $i$ & & & & 22 & \\
\hline $5-198$ & $!$ & 1520 & 138 & $i$ & 1 & & & 125 & \\
\hline $\mathrm{F} \cdot \mathrm{Kq}$ & $!$ & 1523 & .29 & 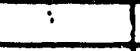 & $!$ & & & 2.2 & \\
\hline 5.199 & & 1524 & 140 & $i$ & 1 & & & $14=$ & \\
\hline Er 100 & 1 & 1527 & 141 & $i$ & - & & & 2 & \\
\hline $5-.50$ & & 1528 & 142 & $!$ & $i$ & & & 125 & \\
\hline$E-2004$ & & 1530 & 143 & 1 & 1 & & & 22 & \\
\hline$[3-200 A$ & & 1531 & 144 & $i$ & 1 & & & 125 & \\
\hline$G-5 y+6 / k 2$ & & $15: 5$ & 145 & $\theta$ & 1 & & & $2 z$ & \\
\hline $5-5 y \leqslant b \mid k=3$ & & 1530 & 146 & 0 & & & & $12=$ & \\
\hline$[s-201$ & & $15 \div 6$ & 147 & 3.6 & & & & 22 & \\
\hline$G-201$ & & 1537 & 148 & 1 & T & & & 125 & \\
\hline$=-202$ & & 1543 & 149 & $i$ & & & & 22 & \\
\hline$G-202$ & & 1544 & 150 & & & & & 123 & \\
\hline$[5 \cdot 203$ & L & 1547 & 151 & & & & & 22 & \\
\hline$\sqrt{5}-203$ & $I$ & 1548 & $15 i$ & & & & & 125 & \\
\hline 5.204 & 1 & 1552 & 153 & i & ) & & & 22 & \\
\hline$\sqrt{2} \cdot 204$ & 1 & 1555 & 154 & & & & & 125 & \\
\hline$E-205$ & & 1257 & 155 & & & & & $z=$ & \\
\hline $5-205$ & 1 & 1530 & 154 & & & & & 130 & \\
\hline$E-20 \dot{6}$ & $i$ & 16001 & 157 & & & & & $\therefore 2$ & \\
\hline $5-i 106$ & $i$ & 1601 & 158 & 1 & & & & 125 & \\
\hline$[5-20]$ & 1 & 16061 & 159 & & & & & 22 & \\
\hline$E-=07$ & 1 & 1627 & 160 & & & & & 125 & \\
\hline$E_{s}-208$ & I & 1611 & 161 & I & & & & 21 & \\
\hline$\sqrt{5}-208$ & L & 1612 & 162 & $E$ & & & & 125 & \\
\hline$E-204$ & 1 & 1615 & 163 & $I$ & 1 & & & 22 & \\
\hline $5-209$ & 6 & 1616 & 164 & $i$ & $T$ & & & 125 & \\
\hline $5-210$ & $11 / 17$ & 1429 & 165 & -4 & & & & 22 & \\
\hline$G-210$ & 1 & 1430 & 166 & & & & & 125 & \\
\hline$E-210 A$ & 5 & 1433 & 167 & 1 & & & & 22 & \\
\hline$G-210 A$ & & 1434 & 165 & 1 & 1 & & & 125 & \\
\hline$G-s, 3$ blk 24 & & 14361 & 169 & 0.0 & & & & 22 & \\
\hline$E$-sys bik 24 & & 1437 & 170 & 0.0 & & & & 125 & \\
\hline$G-211$ & ] & 1420 & 171 & 3.0 & & & & 22 & \\
\hline$G-211$ & 1 & 1451 & 122 & & & & & 125 & \\
\hline
\end{tabular}




\begin{tabular}{|c|}
\hline \\
\hline $\begin{array}{c}\text { alemr: } \\
\text { Lochriom: }\end{array} \frac{W J R C}{740-E}$ \\
\hline
\end{tabular}

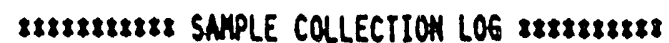

sampler mane(s): R.Pirkle/D, Masdent

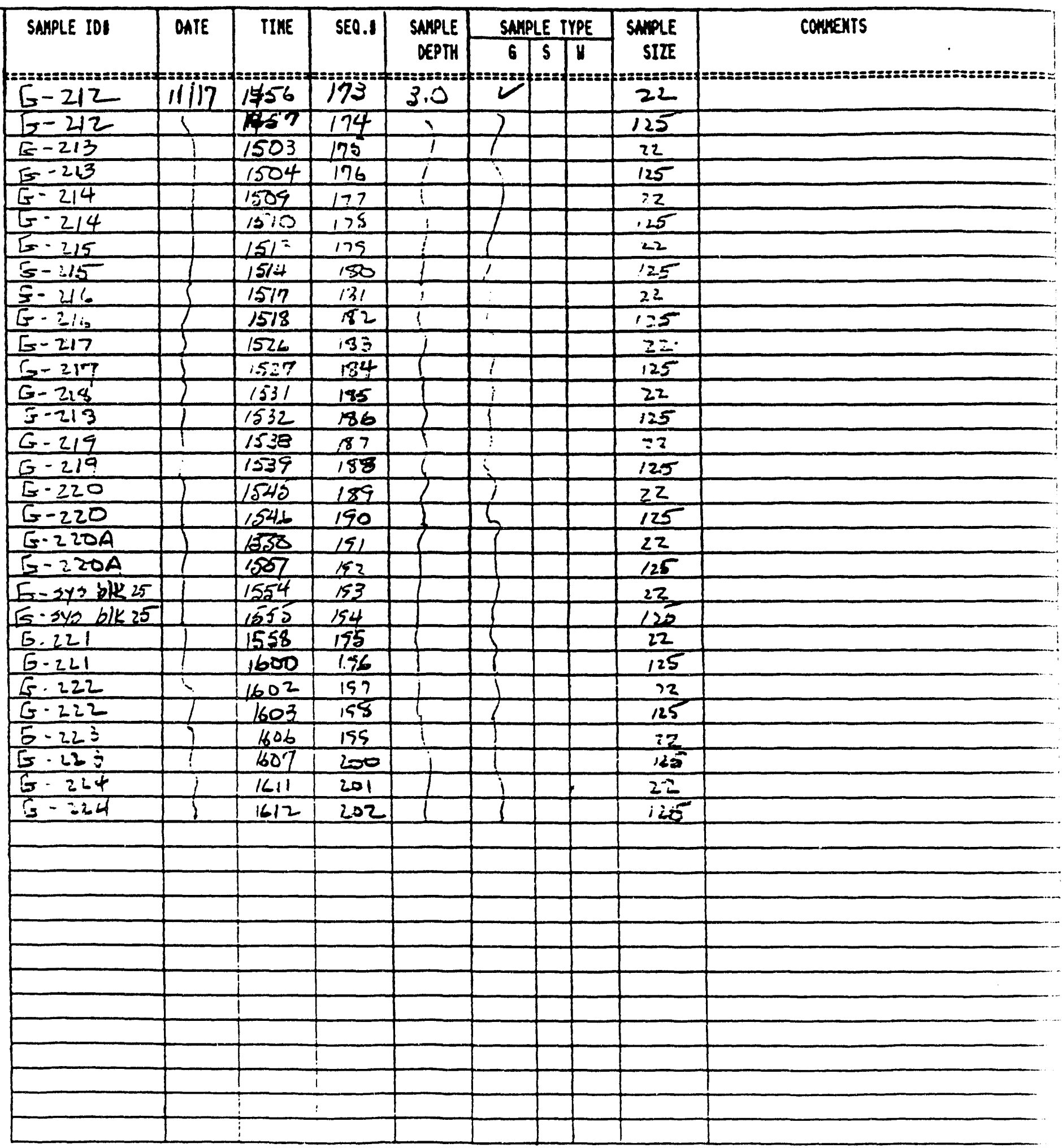


aIEn: $\quad W S R C$

Locarton: $740 \mathrm{G}$

PROJECT(B): TASE 1

PASE: 1 - 1 of:

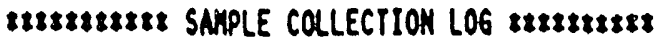

SAMPLER haMe(s): R. R.rkle/ACarr-

\begin{tabular}{|c|c|c|c|c|c|c|c|c|c|}
\hline SAMPLE IOI & DATE & IIME & SEO.1 & SAIPLE & & LE & IYPE & SAMPLE & COMHENTS \\
\hline & :szexs & $=: z=:=:=z$ & sz:z:z:z:z & $\begin{array}{r}\text { DEPTH } \\
==x= \pm= \pm= \pm=\end{array}$ & 6 & 5 & 4 & SILE & 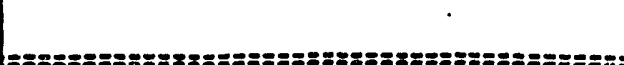 \\
\hline $5 \times 5$ dk 26 & $12 / 2$ & 1030 & 1 & 0.0 & $\checkmark$ & & & 22 & \\
\hline als blk 26 & $\cdot$ & 1031 & 2 & 0.0 & $\checkmark$ & & & 125 & \\
\hline$G-225$ & 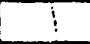 & 1040 & $\Xi$ & $\vdots . .2$ & 1 & & & 22 & \\
\hline$G \cdot 225$ & $i$ & is 41 & -1 & 3.13 & $i$ & & & .25 & \\
\hline$E-i 26$ & i & 1045 & 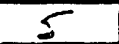 & . & $T$ & & & 22 & \\
\hline$G-: 26$ & $i$ & 1046 & 6 & $\bar{T}$ & $i$ & & & 125 & \\
\hline G.?.L? & 1 & 1052 & 7 & 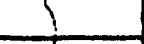 & 1 & & & $\because$. & 1 \\
\hline$C_{5}-227$ & $i$ & 1052 & 8 & I & 1 & & & 25 & \\
\hline $5-228$ & 1 & $10 T$ & 9 & & $!$ & & & 22 & 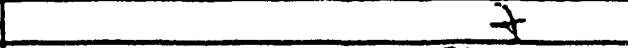 \\
\hline $15-27.9$ & 1 & 1257 & 10 & 1 & $!$ & & & 125 & $=1$ \\
\hline$E-225$ & $i$ & $110^{2}$ & 11 & 1 & $\vdots$ & & & 22 & 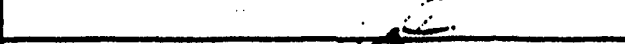 \\
\hline$F=225$ & $\vdots$ & 1104 & .2 & $I$ & - & & & 125 & 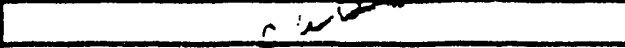 \\
\hline$\sqrt{2}-230$ & $\vdots$ & 1107 & 12 & 1 & $i$ & & & 22 & \\
\hline$F-230$ & 1 & 1108 & 14 & I & : & & & $: 25$ & \\
\hline$[F-220 A$ & $\vdots$ & 1111 & 15 & I & $\vdots$ & & & 22 & \\
\hline$[x-2=1$ & $\vdots$ & 1112 & 16 & & 1 & & & 125 & $\overline{7}$ \\
\hline$[5-221$ & 1 & 1116 & 17 & & 1 & & & 22 & in trenow \\
\hline$r_{2}-231$ & & 117 & 18 & & 1 & & & 125 & \\
\hline $5=-232$ & & 1124 & 19 & & 1 & & & 22 & \\
\hline$r_{2}-232$ & & 1125 & 20 & 4 & 1 & & & 125 & \\
\hline$G-233$ & & 1132 & 21 & 1 & 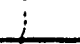 & & & $\div 2$ & \\
\hline$E-233$ & & 1133 & 22 & 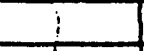 & 1 & & & 125 & \\
\hline$G-5 y \leq b \mid k 27$ & . & 1134 & 23 & 0 & 1 & & & 22 & \\
\hline$E$ - sys bik 27 & 1 & 1135 & 2.4 & 0 & $:$ & & & 125 & \\
\hline$[5 \cdot 234$ & $i$ & 1139 & 25 & 3.0 & 1 & & & 22 & \\
\hline$\sqrt{5}-234$ & $i$ & 1140 & 26 & $\therefore$ & 1 & & & 125 & \\
\hline$\sqrt{3}-225$ & 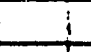 & 1144 & 27 & $i$ & $i$ & & & 22 & \\
\hline G.32\% & I & 1145 & 25 & 1 & & & & 125 & \\
\hline$G-236$ & 1 & 1100 & 24 & 1 & & & & 27 & \\
\hline$E-3-36$ & & 1151 & 30 & $i$ & & & & 12.5 & \\
\hline $5-237$ & & 1156 & 31 & T & T & & & 22 & $8^{\prime N} \mathrm{~N}$ O $\angle F$ N ${ }^{4}$ \\
\hline$\sqrt{2}-237$ & & 1157 & 32 & 1 & $i$ & & & 125 & \\
\hline $5-238$ & & 1159 & 33 & T & $?$ & & & 22 & \\
\hline $6-234$ & & 1200 & 34 & 1 & $i$ & & & 125 & \\
\hline$G-239$ & & 1429 & 35 & 6.0 & i & & & 22 & 1 \\
\hline$E-239$ & & 1430 & 36 & 1 & 1 & & & 125 & \\
\hline$E-240$ & & 7424 & 37 & T & $i$ & & & 22 & $1+1 e$ \\
\hline $5-2+0$ & & 14,5 & 38 & I & $i$ & & & 125 & 10 ort $=$ \\
\hline $5-240 A$ & & 1437 & 39 & & $!$ & & & $2 i$ & $X_{i}{ }^{\prime}$ \\
\hline$E-240 A$ & & 1438 & 40 & & I & & & 115 & co.2 \\
\hline$E \cdot 241$ & & 1440 & 41 & & & & & 22 & 40 \\
\hline$[6-24]$ & I & 1441 & 42 & & & & & 125 & \\
\hline$\sqrt{2}-242$ & 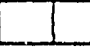 & 1443 & 42 & & & & & 22 & $y$ \\
\hline
\end{tabular}




\begin{tabular}{|c|c|}
\hline & WSRC \\
\hline Locarto" & $740-5$ \\
\hline PROJECT(1): & 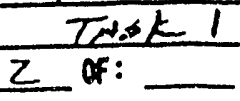 \\
\hline
\end{tabular}

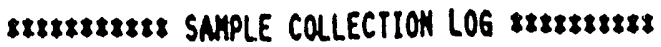

sampler hane(s): R-firkie/A, CAquioz-

\begin{tabular}{|c|c|c|c|c|c|c|c|c|}
\hline SAMPLE IOI & OATE & TIME & SEO.1 & SAMPLE & & FPE & SAMPLE & COMMEHTS \\
\hline 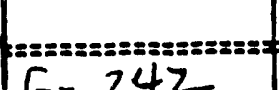 & :zz=zx=: & & & DEPTH & 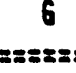 & 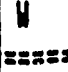 & SILE & 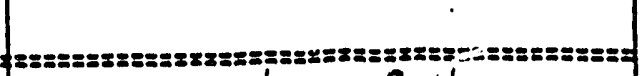 \\
\hline$G-242$ & $12 / 2$ & 1444 & 44 & 6.0 & $V$ & & 125 & $10 \mathrm{ft}$ oct $11=$ \\
\hline $5-5 y=4 k 28$ & $>$ & 1447 & 45 & $!$ & 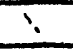 & & 22 & $(\operatorname{sex} 107$ kid \\
\hline$E-2 y=b / k 25$ & 1 & 1448 & 46 & $:$ & 1 & & 125 & \\
\hline $5-243$ & 1 & 1453 & 47 & $\vdots$ & 1 & & 22 & $i$ \\
\hline $5 \cdot 243$ & 1 & 1454 & 48 & $i$ & $!$ & & 125 & $!$ \\
\hline$E-244$ & $I$ & 1457 & 47 & $i$ & $i$ & & 22 & 1 \\
\hline$E-244$ & & 1458 & 50 & $i$ & 1 & & 125 & 1 \\
\hline $5-245$ & & 1505 & 51 & $i$ & 1 & & 22 & 1 \\
\hline $5-745$ & $i$ & 1506 & 52 & $E$ & $T$ & & 125 & \\
\hline $5-246$ & 1 & 1510 & 53 & 5 & 1 & & 22 & $i$ \\
\hline$G-246$ & 1 & $15 i 1$ & 54 & 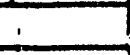 & 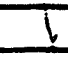 & & 126. & 1 \\
\hline$E-247$ & 1 & 1517 & 5 & $i$ & & & 22 & 11. \\
\hline$G-247$ & & 165 & 50 & 1 & $i$ & & 125 & $\checkmark$ \\
\hline$E-248$ & 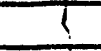 & 1523 & 57 & 1 & $i$ & & 22 & \\
\hline$G-248$ & 1 & 1524 & 53 & 1 & $I$ & & 125 & \\
\hline$\sqrt{5} \cdot 249$ & $T$ & 1527 & 59 & & 1 & & 22 & \\
\hline$\sqrt{E}-249$ & 1 & 1528 & 60 & & $t$ & & 125 & \\
\hline $5-250$ & 1 & 1532 & 61 & & & & 22 & \\
\hline$G-250$ & $T$ & 1533 & 62 & & & & 125 & \\
\hline$E-2 D D A$ & $T$ & 1536 & 65 & & & & 22 & \\
\hline$E-250 A$ & 1 & 1537 & 64 & & $I$ & & 125 & \\
\hline$\sqrt{2} \cdot 251$ & $i$ & 1541 & 65 & 1 & i & & 22 & \\
\hline $5 \cdot 25$ & $T$ & 1542 & $5 i$ & & 4 & & 125 & \\
\hline $5-252$ & $T$ & 1546 & 67 & & & & 22 & \\
\hline$\sqrt{5}-252$ & 1 & 1547 & 68 & & & & 125 & \\
\hline$(5-5 y \leq b \mid k 29$ & & 1504 & 67 & T & & & 22 & \\
\hline$E-3 r \leq b 1 k 2 S$ & & 1565 & 10 & $i$ & & & 125 & \\
\hline & & & & & & & & \\
\hline & & & & & & & & \\
\hline & & & & & & & & \\
\hline & & & & & & & & \\
\hline & & & & & & & & \\
\hline & & & & & & & & \\
\hline & & & & & & & & \\
\hline & & & & & & & & \\
\hline & & & & & & & & \\
\hline & & & & & & & & \\
\hline & & & & & & & & \\
\hline & & & & & & & & \\
\hline & & & & & & & & \\
\hline & & & & & & & & \\
\hline & & $!$ & & & & & & \\
\hline & & $i$ & & & & & & \\
\hline
\end{tabular}


PAGE: _— OF:

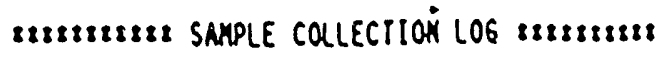

SAMPLER XAKE(S): Kab/AaC

\begin{tabular}{|c|c|c|c|c|c|c|c|c|c|}
\hline \multirow{2}{*}{ 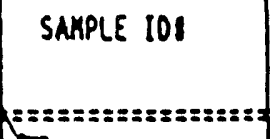 } & \multirow[t]{2}{*}{ OATE } & \multirow[t]{2}{*}{ IIME } & \multirow[t]{2}{*}{ SEO.1 } & \multirow{2}{*}{$\begin{array}{r}\text { SAMPLE } \\
\text { DEPIH } \\
=:=:=:=:=\end{array}$} & \multicolumn{3}{|c|}{ SAMPLE TYPE } & \multirow{2}{*}{$\begin{array}{l}\text { SAMPLE } \\
\text { SILE } \\
\text { : : ::::=: }\end{array}$} & \multirow[t]{2}{*}{ COMnENTS } \\
\hline & & & & & 6 & $S$ & 4 & & \\
\hline $740-G-S B .32$ & 5.29 .91 & 1440 & 1 & $-0-$ & $\checkmark$ & & & 22 & \\
\hline $740 \cdot G-5 B-32$ & & 1442 & 2 & $-0=$ & & & & 125 & \\
\hline $740.5-262$ & & 1444 & 3 & $3^{\prime}$ & & & & 22 & $\dot{-}$ \\
\hline$G .262$ & & 1445 & 9 & & & & & 125 & \\
\hline 6.263 & & 1447 & 5 & & & & & 1 & \\
\hline $6.24,3$ & & 1948 & 6 & & & & &. & \\
\hline 6.264 & & 1452 & 7 & & & & & 1 & \\
\hline 6.264 & & 1454 & 8 & & & & & 1 & \\
\hline $5-265$ & & 1456 & C & & & & & $T$ & \\
\hline $5-2 / 5$ & & 1457 & 10 & 1 & & & & I & \\
\hline$G 2 \ln$ & & 1458 & II & 1 & & & & & \\
\hline$G .216$ & & 1500 & 12 & & & & & & \\
\hline 6.267 & & 1503 & 13 & & & & & 1 & \\
\hline 6.267 & & 1504 & 14 & & & & & 1 & \\
\hline 6.2108 & & 1506 & 15 & & & & & $T$ & \\
\hline 6.268 & & 1507 & 16 & & & & & 1 & \\
\hline G.269 & & 1510 & 17 & & & & & $!$ & \\
\hline$G-269$ & & 1511 & 18 & & & & & 1 & \\
\hline G-269A & & 1513 & 19 & & & & & I & \\
\hline$G-269 \theta$ & & 1514 & 20 & $\sqrt{2}$ & & & & 1 & \\
\hline G.SB-33 & & 1515 & 21 & -0 & & & & $i$ & \\
\hline G.SB.33 & & 1516 & 22 & $-0-$ & & & & 1 & \\
\hline G.270 & & 1517 & 23 & 31 & & & & t & \\
\hline 6.270 & 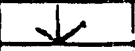 & 1518 & 24 & & & & & 1 & \\
\hline$G .271$ & $5-30-91$ & 843 & 25 & 1 & 1 & & & $i$ & \\
\hline 6.271 & & 844 & 26 & 1 & 1 & & & $i$ & \\
\hline$G .272$ & & 845 & 27 & $i$ & & & & $T$ & \\
\hline$G .272$ & & 847 & 28 & $i$ & & & & $!$ & \\
\hline$G .273$ & & 848 & 29 & $i$ & & & & $i$ & \\
\hline$G .273$ & & 849 & 30 & & & & & $i$ & - \\
\hline G.274 & & 850 & 31 & & & & & $i$ & \\
\hline 6.274 & & 851 & 32 & & & & & $\vdots$ & $\therefore$ \\
\hline$G .275$ & & 854 & 33 & & & & & $i$ & \\
\hline 6.275 & & 855 & 34 & & & & & $\vdots$ & \\
\hline$G .276$ & & 901 & 35 & & & & & $i$ & \\
\hline G.276 & & 902 & 36 & & & & & 1 & \\
\hline G.277 & & 918 & 37 & & & & & 1 & \\
\hline 6.277 & & 920 & 38 & & & & & $i$ & \\
\hline 6.278 & & 1204 & 39 & & 1 & & & 1 & \\
\hline G.278 & & 1205 & 40 & & 1 & & & 1 & \\
\hline 6.279 & & 1210 & 41 & & $!$ & & & $i$ & \\
\hline 6279 & & 1212 & 42 & & $i$ & & & 1 & \\
\hline 0.2799 & $v$ & 1214 & +3 & $v$ & $v$ & & & $w$ & \\
\hline
\end{tabular}


RICROSEEPS $L I O$.

C.IEXT: WSRS/Dous Wyatt:

Locallow: handfiLl Extension PROJECT(1): $740 . G$

PAGE: — OF:

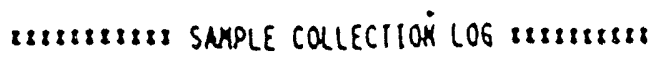

SAKPLER MAKE(S):

\begin{tabular}{|c|c|c|c|c|c|c|c|c|c|}
\hline SAXPLE 101 & DATE & IIME & SEO.1 & SAMPLE & & PE & & SAXPLE. & COHHEXTS \\
\hline 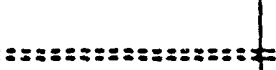 & $:=: 2 x=8=$ & & $::=:: s:=$ & DEPIH & 6 & $s$ & $y$ & SILE & \\
\hline $740 \cdot G-279 A$ & 5.30 & 1215 & $\Delta 4$ & 3 & $\checkmark$ & & . & 125 & 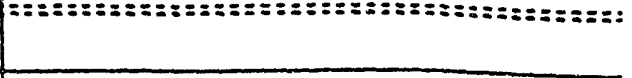 \\
\hline $740-G .3 B \cdot 34$ & & 1218 & 45 & .0 & & & & 22 & \\
\hline $740 \cdot G-58.24$ & & 1219 & 46 & $-0-$ & & & & 125 & $\dot{-}$ \\
\hline$G-280$ & & 1220 & 47 & $3^{\prime}$ & & & & 22 & \\
\hline$G-280$ & & 1221 & 48 & & & & & & \\
\hline$G .281$ & $i$ & 1224 & 49 & & & & & 1 & \\
\hline$G .281$ & & 1225 & 50 & & & & & $i$ & \\
\hline$G .282$ & & 1230 & 51 & & & & & 1 & \\
\hline 3.282 & 1 & 1232 & 52 & & & & & I & \\
\hline G.723 & $T$ & 1237 & 53 & & & & & 1 & \\
\hline$=-283$ & & 1238 & 54 & & & & & 1 & \\
\hline$\xi .284$ & 1 & 1243 & 55 & 1 & $i$ & & & $i$ & \\
\hline$G-224$ & 1 & 1244 & 56 & $i$ & 1 & & & $!$ & \\
\hline$G .285$ & 1 & 1249 & 57 & 1 & & & & $i$ & \\
\hline G.285 & $!$ & 1250 & 58 & $i$ & 1 & & & 1 & \\
\hline$G .286$ & 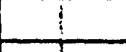 & 1253 & 59 & + & & & & 1 & \\
\hline$G .286$ & 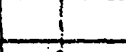 & 1254 & 60 & 1 & & & & & \\
\hline$G-287$ & $i$ & 1258 & 61 & & & & & & \\
\hline $6-287$ & $i$ & 1259 & 62 & & & & & & \\
\hline$G=288$ & 1 & 1304 & 63 & & & & & $!$ & \\
\hline$G \cdot 288$ & 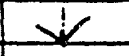 & 1305 & let & $-k$ & $\boldsymbol{x}$ & & & I & \\
\hline & & & & & & & & & \\
\hline & & & & & & & & 1 & \\
\hline & & & & & & & & $\vdots$ & \\
\hline & & & & & & & & $\vdots$ & \\
\hline & & 1 & & & & & & $!$ & \\
\hline & & & & & & & & $!$ & \\
\hline & & 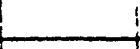 & &. & & & & $!$ & \\
\hline & & & & & & & & 1 & \\
\hline & & 1 & & & & & & 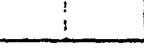 & \\
\hline & & & & & & & & 1 & \\
\hline & & & & & & & & $!$ & - \\
\hline & & & & & & & & & \\
\hline & & & & & & & & & \\
\hline & & & & & & & & & \\
\hline & & & & & & & & & \\
\hline & & & & & & & & 1 & \\
\hline & & $!$ & & & & & & I & \\
\hline & & & & & & & & & \\
\hline & & & - & & & & & 1 & \\
\hline & & & & & & & & 1 & \\
\hline & & $!$ & & & & & & $i$ & \\
\hline & & & & & & & & 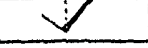 & \\
\hline
\end{tabular}


HICROSEEPS LTD.

aIEn: WSRC

Lochillow: $740-G-L_{\text {avafdl }}$

PROJECT(B): TASK

PAGE: 1 of: 2

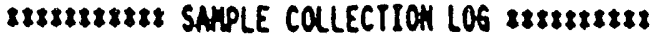

sampler name(s): AGC +JPajs

\begin{tabular}{|c|c|c|c|c|c|c|c|c|c|}
\hline SAMPLE IOI & DATE & TIME & SEQ.1 & SAMPLE & SAMPL & LE T & YPE & SMPLE & COAHENTS \\
\hline & =:28z=:z= & :E=:=ะz: & & DEPTH & 6 & 5 . & $\mid=$ & SIZE & 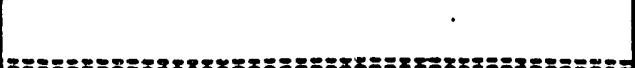 \\
\hline $740-6 \cdot 1$ & $\mid-8-91$ & & 1 & ift & & $=27$ & & & \\
\hline-2 & 1 & & 2 & $<$ & & & & & \\
\hline-3 & ! & & 3 & C & & & & & \\
\hline-4 & $!$ & & 4 & 7 & & & & & \\
\hline-5 & & & 5 & & & & & & \\
\hline-6 & & & 6 & $\because$ & & & & & \\
\hline-7 & & & 7 & & & & & & \\
\hline-8 &. & & 8 & & & & & & \\
\hline-9 & & & 9 & & & & & & \\
\hline-10 & $i$ & & 10 & $!$ & & & & & \\
\hline$-10 \pi$ & & & 11 & & & & & $\cdot$ & \\
\hline-11 & • & & 12 & & & & & & \\
\hline-12 & $\vdots$ & & 13 & $\therefore$ & & & & & \\
\hline $7 \overline{3}$ & $i$ & & 14 & & & & & & \\
\hline-14 & $\vdots$ & & 15 & & & 1 & & & \\
\hline $2+16-15$ & $|-9-9|$ & & 16 & $i$ & & & & & \\
\hline-16 & & & 17 & 7 & & 1 & & & \\
\hline-17 & & & 18 & & & & & & \\
\hline-18 & & & 19 & & & & & & \\
\hline-19 & & & 20 & & & & & & \\
\hline-20 & & & 21 & $!$ & & & & & \\
\hline$-20 A$ & & & 42 & $\div$ & & & & & \\
\hline-21 & & & 23 & & & $i$ & & & \\
\hline-22 & & & 24 & & & & & & \\
\hline-23 & & & 25 & $i$ & & 1 & & & \\
\hline-24 & & & 2.5 & & & & & & \\
\hline-25 & & & 27 & & & 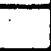 & & & \\
\hline-26 & & & 28 & & & $i$ & & & \\
\hline .27 & & & $2 ?$ & $i$ & & 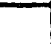 & 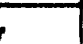 & & \\
\hline-27 & & & 30 & $!$ & & $i$ & & & \\
\hline-29 & & & 31 & $\bar{\square}$ & & $\therefore$ & & & \\
\hline-30 & & & 32 & & & $i$ & & & \\
\hline$-30 A$ & & & 33 & 1 & & & & & \\
\hline .31 & & & 34 & 1 & & $T$ & & & \\
\hline-32 & & & 35 & & & & & & \\
\hline-33 & & & 36 & 1 & & 7 & & & \\
\hline-34 & & & 37 & 7 & & & & & \\
\hline .35 & & & 38 & & & & & & \\
\hline-36 & & & 39 & & & $T$ & & & \\
\hline-37 & & & 40 & $\because$ & & 1 & & & \\
\hline-38 & & & $4 i$ & $i$ & & $\because$ & & & \\
\hline .39 & & & $4 i$ & . & & $!$ & & & \\
\hline .41 & & & 42 & & & & & & \\
\hline
\end{tabular}


nICROSEEPS LTO.

aIm: WSRC

Locarlon: $740-G-2 A \cup 0 f .1$

PROJECT(1): TASK

PAGE: 2 of: 2

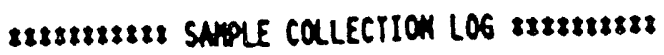

SAPPLER MaAE(S): A.G.C.JP+JS

\begin{tabular}{|c|c|c|c|c|c|c|c|c|c|}
\hline \multirow{2}{*}{ 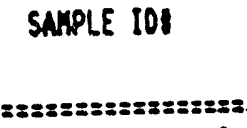 } & \multirow{2}{*}{ OATE } & \multirow{2}{*}{ TIME } & \multirow{2}{*}{ SEO.1 } & \multirow{2}{*}{$\begin{array}{l}\text { SAMPLE } \\
\text { DEPTH }\end{array}$} & \multicolumn{3}{|c|}{ SMPPLE TPPE } & \multirow{2}{*}{$\begin{array}{c}\text { SAPPLE } \\
\text { SIIEE } \\
\text { z2:3z:Zz: }\end{array}$} & \multirow{2}{*}{ 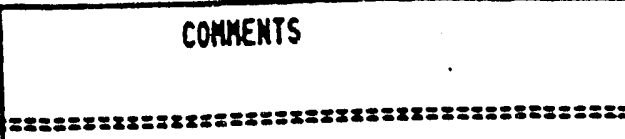 } \\
\hline & & & & & $\begin{array}{c}6 \\
=3 x \leq ?\end{array}$ & 5 & $y$ & & \\
\hline$\frac{740-6-40 A}{-41}$ & -9.91 & & 44 & $1 \mathrm{ft}$ & & V & & & \\
\hline $\begin{array}{l}-41 \\
-42\end{array}$ & & & 45 & & & 1 & & & \\
\hline$\frac{-42}{-43}$ & & & 46 & & & $\square$ & & & \\
\hline$\frac{-43}{-44}$ & & & $\frac{47}{48}$ & & & & & & \\
\hline $\begin{array}{l}-44 \\
-45 \\
\end{array}$ & & & $\frac{48}{49}$ & & & & & & \\
\hline-46 & & & 50 & & & & & & \\
\hline$\frac{-47}{.48}$ & & & 51 & & & 1 & & & \\
\hline$\frac{.48}{-49}$ & & & 52 & & & $!$ & & & \\
\hline $\begin{array}{l}-49 \\
-50 \\
\end{array}$ & & & 53 & & & & & & \\
\hline$-50 \mathrm{~A}$ & & & $\frac{54}{55}$ & & & $\vdots$ & & & \\
\hline-51 & & & 56 & & & & & & \\
\hline$\frac{-52}{53}$ & & & 5 & & & & & & \\
\hline$\frac{-53}{54}$ & & & 58 & & & 1 & & & \\
\hline$\frac{54}{-55}$ & & & 59 & & & 1 & & & \\
\hline $\begin{array}{l}\frac{-55}{-56} \\
\end{array}$ & & & 60 & & & & & & \\
\hline$\frac{-56}{-57}$ & & & $\frac{61}{62}$ & & & & & & \\
\hline$\frac{-21}{-58}$ & & & $\frac{62}{63}$ & & & $\frac{1}{\vdots}$ & & & \\
\hline$\frac{-59}{-60}$ & & & 64 & & & & & & \\
\hline$\frac{-60}{-60 A}$ & & & 65 & & & & & & \\
\hline$\frac{-60 A}{-6 !}$ & & & 66 & $:$ & & 1 & & & \\
\hline-62 & & & $\frac{67}{68}$ & & & - & & & \\
\hline-63 & & & $\frac{80}{89}$ & & & & & & \\
\hline$\frac{-64}{-65}$ & & & 70 & & & & & & \\
\hline$\frac{-65}{-66}$ & & & 71 & & & & & & \\
\hline$\frac{-66}{-67}$ & & & 72 & & & & & & \\
\hline$\frac{-67}{-68}$ & & & $\frac{73}{74}$ & $\vdots$ & & & & & \\
\hline $\begin{array}{l}-68 \\
-69 \\
\end{array}$ & & & $\frac{17}{75}$ & $\therefore$ & & & & & \\
\hline-20 & & & 76 & & & & & & \\
\hline$-28 \mathrm{~A}$ & & & 77 & $i$ & & & & & \\
\hline-71 & & & 78 & & & & & & \\
\hline$\frac{-72}{72}$ & & & 79 & & & - & & & \\
\hline$\frac{-73}{-74}$ & & & 50 & & & & & & \\
\hline$\frac{-74}{-75}$ & & & 81 & & & - & & & \\
\hline $\begin{array}{l}-75 \\
-76 \\
\end{array}$ & & & $\frac{82}{83}$ & & & $i$ & & & \\
\hline-77 & & & 84 & & & 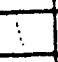 & & & \\
\hline$\frac{-78}{-79}$ & & & 85 & & & - & & & \\
\hline-79 & & & 86 & & & & & & \\
\hline
\end{tabular}


MICPOSEEPS LTO.

aIEn: WSRC

Locarion: $740 G-$ Landell

PROJECT(1):- Tark $* 1$

PAGE: 3 of: 7

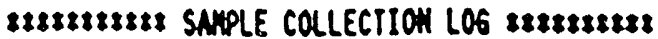

SAMPLER MAKE(S): $A G C+J P+J S$

\begin{tabular}{|c|c|c|c|c|c|c|c|c|c|}
\hline SAKPLE IDI & DATE & IIME & SEQ.1 & SAMPLE & $\operatorname{sen} P$ & LE T & & SAMPLE & COAnENTS \\
\hline & & & & DEPTH & 6 & 5 & $y$ & SIIE & \\
\hline 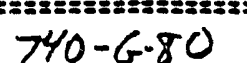 & $\begin{array}{l}=z 2 x 8 z=3 \\
|-9-9|\end{array}$ & & :mez:= & & & $=2=8$ & & & 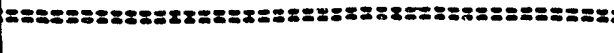 \\
\hline$-80 A$ & & & $\frac{81}{58}$ & & & 1 & & & \\
\hline-81 & & & 89 & 1 & & T & & & \\
\hline-82 & & & 90 & $T$ & & $T$ & 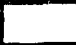 & & \\
\hline-83 & & & 91 & $\therefore$ & & $I$ & E & & \\
\hline-84 & & & 92 & $i$ & & I & & & \\
\hline-85 & & & 93 & & & I & & & \\
\hline-86 & & & 94 & $!$ & & I & & & \\
\hline-87 & & & 95 & & & I & & & \\
\hline-88 & & & 96 & 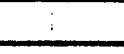 & & 1 & & & \\
\hline-89 & & & 97 & $i$ & & 1 & & $\dot{-}$ & \\
\hline-90 & & & 98 & $\bar{I}$ & & $i$ & & & \\
\hline$-90 \mathrm{~A}$ & & & 99 & 1 & & $T$ & & & \\
\hline-91 & & & 100 & $i$ & & - & - & & \\
\hline-92 & & & 101 & 1 & & 1 & & & \\
\hline-93 & 1 & & 102 & I & & I & & & \\
\hline-94 & & & 103 & ! & & $i$ & & & \\
\hline .95 & & & 104 & I & & I & E & & \\
\hline-96 & 1 & & 105 & + & & I & & & \\
\hline .97 & 1 & & 106 & 1 & & 1 & & & \\
\hline-98 & & & 107 & 1 & & 7 & & & \\
\hline-99 & $1+0,91$ & & 108 & $I$ & & $I$ & & & \\
\hline-100 & & & 109 & $I$ & & 1 & & & \\
\hline$-100 A$ & & & 110 & $i$ & & II & & & \\
\hline-101 & & & 111 & 1 & & 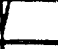 & & & \\
\hline-102 & & & 112 & 1 & & 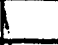 & & & \\
\hline-103 & & & 113 & 1 & & 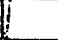 & & & \\
\hline-104 & & & 114 & I & & 1 & & & \\
\hline-105 & & & 115 & $\bar{i}$ & & 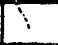 & & & \\
\hline-106 & & & 116 & $i$ & & 1 & & & \\
\hline .107 & & & 117 & 1 & & 1 & & & \\
\hline-108 & & & 118 & $!$ & & $!$ & & & \\
\hline-109 & & & 119 & 1 & & 1 & & & \\
\hline-110 & & & 120 & 1 & & $T$ & & & \\
\hline$-160 \mathrm{~A}$ & & & 121 & $i$ & & 1 & & & \\
\hline-111 & & & 122 & $\because$ & & 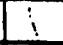 & & & \\
\hline$-1 / 2$ & & & 123 & 1 & & 1 & 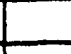 & & \\
\hline-113 & & & 124 & 1 & & 1 & & & \\
\hline-114 & & & 125 & $I$ & & 1 & & & \\
\hline-115 & & & 126 & $i$ & & $:$ & & & \\
\hline-116 & & & 127 & 1 & & $:$ & & & \\
\hline-117 & & & 128 & $!$ & & 1 & & & \\
\hline$-1 / 8$ & & & 129 & 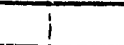 & & : & & & \\
\hline
\end{tabular}


HICROSEEPS LIT.

aIEnT: WSRC

Lochilow: $740-6$ Lonofil

PROJECI(1): TASK',

PAGE: 4 OF: 2

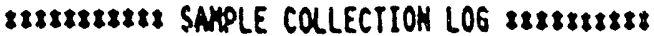

SAMPLER MaME(S): $A G C+J P+d S$

\begin{tabular}{|c|c|c|c|c|c|c|c|c|c|}
\hline SAMPLE IDI & OATE & TIKE & SEO.1 & SMPLE & SMMP & LE T & & SAPPLE & COMHENTS \\
\hline 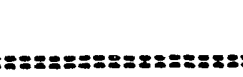 & :zz2z:=2: & $=: 2=: 28$ & $z=2 \geq z 2=28$ & $\begin{array}{c}\text { DEPTH } \\
=2=2=2 \times x z\end{array}$ & $\begin{array}{c}6 \\
:=x=28\end{array}$ & $\begin{array}{c}5 \\
:=28\end{array}$ & $\sum_{z=: 2}$ & SIZE & 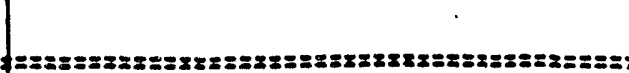 \\
\hline $240-6-119$ & $10-10$ & & 130 & $\angle A$ & & $V$ & & & \\
\hline-120 & , & & 131 & & & $t$ & & & \\
\hline-121 & & & 132 & 7 & & L & & & \\
\hline-122 & & & 133 & - & & $L$ & & & \\
\hline-123 & & & 134 & 1 & & E & & & \\
\hline-124 & & & 135 & $T$ & & F & & & \\
\hline-125 & & & 136 & & & E & & & \\
\hline-126 & & & 137 & $i$ & & 1 & & & \\
\hline-127 & & & 138 & $i$ & & 1 & & & \\
\hline-128 & & & 139 & 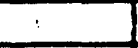 & & $T$ & & & \\
\hline-129 & & & 140 & & & 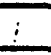 & & $\dot{-}$ & \\
\hline$-129 \cdot A$ & & & 141 & & & 1 & & & \\
\hline-130 & & & 142 & & & 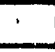 & & & \\
\hline 232 & & & 143 & & & 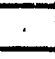 & & & \\
\hline$-131-4$ & $i$ & & 144 & 1 & & $i$ & & & \\
\hline-132 & & & 145 & 1 & & 1 & & & \\
\hline-133 & & & 146 & 1 & & $i$ & & & \\
\hline-134 & & & (4) & $i$ & & I & & & \\
\hline-135 & & & 148 & 7 & 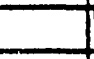 & $E$ & & & \\
\hline .136 & & & 149 & $i$ & & $i$ & & & \\
\hline-137 & & & 150 & $i$ & & 1 & & & \\
\hline-138 & & & 151 & $i$ & & $\vdots$ & & & \\
\hline 139 & 1 & & 152 & & & $T$ & & & \\
\hline-140 & $\vdots$ & & 153 & $\vdots$ & & $\vdots$ & & & \\
\hline$-140 A$ & $:$ & & 154 & $i$ & & $!$ & & & \\
\hline-141 & L/21 & & 155 & $!$ & & 1 & & & \\
\hline-142 & & & 156 & & & 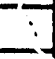 & & & \\
\hline-143 & & & 157 & & & $i$ & & & \\
\hline-144 & & & 158 & $\vdots$ & & $i$ & & & \\
\hline-145 & & & 159 & $\vdots$ & & 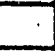 & & & \\
\hline-146 & & & 160 & & & 1 & & & \\
\hline-142 & & & 161 & & & 1 & & & \\
\hline-148 & & & 162 & 1 & & & & & \\
\hline-149 & & & 163 & 1 & & $i$ & & & \\
\hline-150 & & & 164 & $\vdots$ & & 3 & & & \\
\hline-151 & & & 165 & & & $i$ & & & \\
\hline-152 & & & 166 & 1 & & $i$ & & & \\
\hline-153 & & & 16) & $i$ & & 1 & & & \\
\hline-154 & & & 168 & $\vdots$ & & 1 & & & \\
\hline-155 & & & 169 & $i$ & & 1 & & & \\
\hline-1554 & & & 170 & 1 & & $i$ & & & \\
\hline-156 & & & $17 i$ & 1 & & $:$ & & & \\
\hline-15 & & & 172 & & & $i$ & & & \\
\hline
\end{tabular}


MICROSEEPS LTD.

arem: WSRC

LOCATIOH: $740-G$ LAOOAII

PROJECT(B): TASKA1

PAGE: $\frac{7}{5}$ OF: 7

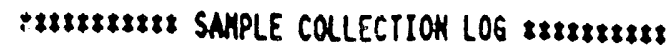

SAMPLER MAME(S): AGC+.Po JS

\begin{tabular}{|c|c|c|c|c|c|c|c|c|c|}
\hline \multirow{2}{*}{$\begin{array}{l}\text { SARPLE IOI } \\
\text { ::8:=:::=:::=:=:z }\end{array}$} & \multirow{2}{*}{$\begin{array}{l}\text { OATE } \\
\text { :z:=:= }\end{array}$} & \multirow[t]{2}{*}{ TIME } & \multirow[t]{2}{*}{ SEO.1 } & \multirow{2}{*}{$\begin{array}{l}\text { SAMPLE } \\
\text { DEPTH }\end{array}$} & \multicolumn{3}{|c|}{ SAMPLE IYPE } & \multirow{2}{*}{$\begin{array}{c}\text { SANPLE } \\
\text { SIIE } \\
\text { : :E:E:E: }\end{array}$} & \multirow[t]{2}{*}{ COHNENTS } \\
\hline & & & & & $=\begin{array}{c}6 \\
=3 x z=\end{array}$ & $=5$ & N & & \\
\hline $740-6-158$ & 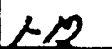 & & 173 & $1 \mathrm{ft}$ & & $\checkmark$ & & & 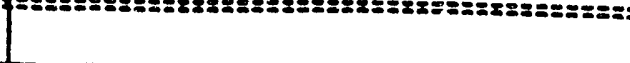 \\
\hline-159 & 1 & & 174 & & & $\zeta$ & & & \\
\hline-160 & $\xi$ & & 175 & 7 & & 1 & & & \\
\hline$-160 A$ & 1 & & 176 & 1 & & $i$ & & & \\
\hline-161 & 1 & & 127 & $i$ & & $i$ & & & \\
\hline$\frac{-162}{-163}$ & $i$ & & 178 & $!$ & & $i$ & & & \\
\hline$\frac{-163}{-164}$ & 1 & & 179 & $i$ & & I & & & \\
\hline$\frac{-164}{-165}$ & I & & 180 & $!$ & & 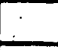 & & & \\
\hline$\frac{-165}{-166}$ & $!$ & & 181 & $\vdots$ & & $I$ & & & \\
\hline$\frac{-166}{-167}$ & 1 & & $\frac{182}{183}$ & $\vdots$ & & ti & & & \\
\hline-168 & & & 184 & ! & & & & & \\
\hline-169 & $\therefore$ & & 185 & $i$ & & - & & & \\
\hline-170 & & & 186 & $T$ & & $\because$ & & & \\
\hline$-170 A$ & $i$ & & 187 & $i$ & & 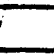 & & & \\
\hline-171 & & & 188 & $T$ & & $\overline{1}$ & & & \\
\hline-172 & & & 189 & 1 & & 7 & & & \\
\hline-173 & I & & 190 & $T$ & & 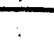 & & & \\
\hline-124 & $I$ & & 191 & 1 & & & & & \\
\hline-175 & 1 & & 192 & $T$ & & 1 & & & \\
\hline-176 & 1 & & 193 & $I$ & & $\because$ & & & \\
\hline-127 & $I$ & & 194 & $I$ & & 1 & & & \\
\hline-178 & 1 & & 195 & $I$ & & $!$ & & & \\
\hline-179 & 1 & & 196 & : & & 1 & & & \\
\hline$\frac{-150}{-1500}$ & $!$ & & 192 & 1 & & 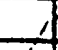 & & & \\
\hline$\frac{-130 A}{-181}$ & $i$ & & 198 & 1 & & 1 & & & \\
\hline$\frac{-181}{-182}$ & $\frac{1}{1}$ & & 199 & $\vdots$ & & 1 & & & \\
\hline-183 & & & $\frac{200}{201}$ & $\frac{1}{1}$ & & -1 & & & \\
\hline-184 & 1 & & 202 & $i$ & & -7 & & & \\
\hline-185 & I & & 203 & $!$ & & 7 & & & \\
\hline-186 & I & & 204 & 1 & & 1 & & & \\
\hline-187 & 1 & & 205 & 1 & & i & & & \\
\hline-188 & $I$ & & 206 & i & & 1 & & & \\
\hline-189 & I & & 207 & $!$ & & 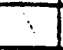 & & & \\
\hline 190 & I & & 207 & $T$ & & $i$ & & & \\
\hline$-190 \mathrm{~A}$ & & & 209 & $T$ & & $I$ & & & \\
\hline-191 & & & 210 & $T$ & & $!$ & & & \\
\hline-192 & 1 & & 211 & 1 & & $!$ & & & \\
\hline-193 & & & 212 & $T$ & & $\bar{\vdots}$ & & & \\
\hline-194 & & & 213 & $i$ & & $!$ & & & \\
\hline$\frac{-195}{-196}$ & 1 & & $\frac{214}{115}$ & & & & & & \\
\hline & & & 215 & 1 & & $!$ & & & \\
\hline
\end{tabular}


aIEm: WSRC

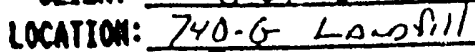

PROJECT(1): TASK

PneE: 6 of: 7

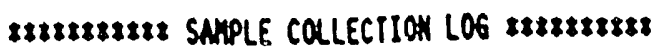

SAMPLER MAME(S): AGCAJP+JS

\begin{tabular}{|c|c|c|c|c|c|c|c|c|c|}
\hline \multirow{2}{*}{ 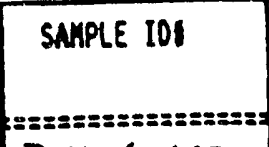 } & \multirow[t]{2}{*}{ DATE } & \multirow[t]{2}{*}{ TIME } & \multirow[t]{2}{*}{ SEQ.1 } & \multirow{2}{*}{$\begin{array}{l}\text { SAMPLE } \\
\text { DEPTH }\end{array}$} & \multicolumn{3}{|c|}{ SAMPLE TYPE } & \multirow{2}{*}{ 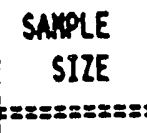 } & \multirow{2}{*}{ 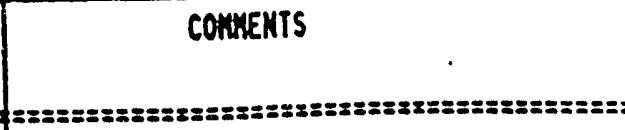 } \\
\hline & & & & & $=:=2 x=$ & $\begin{array}{c}S \\
:=2:\end{array}$ & $y$ & & \\
\hline $240-6-197$ & $1-12$ & & 216 & $1 \mathrm{ft}$ & & $\checkmark$ & & & \\
\hline$\frac{-198}{-199}$ & & & 217 & & & $I$ & & & \\
\hline$\frac{-199}{-200}$ & & & 218 & $i$ & & t & & & \\
\hline$\frac{-200}{900}$ & & & 219 & & & 1 & & & \\
\hline$-200 A$ & & & 220 & & & $!$ & & & \\
\hline-201 & & & 221 & & & $T$ & & & \\
\hline .202 & & & 2.12 & & & $T$ & & & \\
\hline .203 & & & 223 & $!$ & & $T$ & & & \\
\hline-204 & & & 224 & $\cdot$ & & 7 & & & \\
\hline-205 & & & 225 & $i$ & & 7 & & & \\
\hline-205 & & & 226 & $i$ & & & & $\cdot$ & \\
\hline$-2,07$ & & & 227 & & & 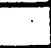 & & & \\
\hline$-20 \delta$ & 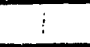 & & 228 & $i$ & & $i$ & & & \\
\hline$-20 ?$ & $!$ & & 229 & $i$ & & $\bar{\vdots}$ & & & \\
\hline-210 & & & 230 & $i$ & & 5 & & & \\
\hline-2104 & & & 231 & $!$ & & $\bar{l}$ & & & \\
\hline$-211^{\circ}$ & . & & 232 & $\vdots$ & & $i$ & & & \\
\hline-212 & $T$ & & 233 & 1 & & $T$ & & & \\
\hline-213 & $T$ & & 234 & $!$ & & 1 & & & \\
\hline .214 & ? & & 235 & & & t & & & \\
\hline-25 & : & & 236 & & & 7 & & & \\
\hline-216 & $T$ & & 237 & $i$ & & 7 & & & \\
\hline$-2 \sqrt{7}$ & 7 & & 238 & $T$ & & $T$ & & & \\
\hline-215 & & & 239 & & & $i$ & & & \\
\hline 214. & $!$ & & 240 & $?$ & & $\vdots$ & & & \\
\hline 200 & 1 & & 241 & 1 & & $i$ & & & \\
\hline$-225 A$ & & & 242 & $!$ & & 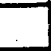 & & & \\
\hline 221 & & & 243 & $\vdots$ & & $!$ & & & \\
\hline 222 & $T$ & & 244 & $i$ & & 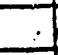 & & & \\
\hline 223 & $i$ & & 245 & $\vdots$ & & $i$ & & & \\
\hline 224 & 1 & & 246 & & & $\div$ & & & \\
\hline 225 & $1-14$ & & 247 & 1 & & $i$ & & & \\
\hline 226 & 1 & & 248 & $i$ & & $\therefore$ & & & \\
\hline 20 & & & 249 & $\because$ & & $\therefore$ & & & \\
\hline 228 & $i$ & & 250 & 1 & & & & & \\
\hline 229 & 1 & & 251 & $!$ & & $\therefore$ & & & \\
\hline 230 & 1 & & 252 & $\div$ & & & & & \\
\hline $230 A$ & 1 & & $25 ?$ & 1 & & & & & \\
\hline 231 & 1 & & 254 & 1 & & & & & \\
\hline 232 & & & 253 & $i$ & & & & & \\
\hline$\frac{233}{234}$ & 1 & & 256 & $T$ & & - & & & \\
\hline$\frac{234}{035}$ & & & 25 & 5 & & - & & & \\
\hline 235 & & & $2 \pi x$ & & & & & & \\
\hline
\end{tabular}


MICROSEEPS LID.

arem: WSRC

Locantion: 240.6 tand $P_{11}$

Prouect(b): TASK $K^{\circ}$

PAGE: 2 of: $?$

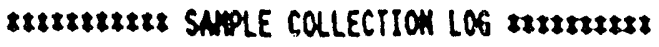

SAMPLER MaMe(S): $A G C+\sqrt{ } P+\downarrow S$

\begin{tabular}{|c|c|c|c|c|c|c|c|c|c|}
\hline \multirow{3}{*}{ SAMPLE IOI } & \multirow[t]{2}{*}{ DATE } & \multirow[t]{2}{*}{ TIME } & \multirow[t]{2}{*}{ SEQ.1 } & \multirow{2}{*}{$\begin{array}{l}\text { SAHPLE } \\
\text { DEPTH }\end{array}$} & \multicolumn{3}{|c|}{ SAMPLE TYPE } & \multirow{3}{*}{$\begin{array}{l}\text { SAPPE } \\
\text { SIIE }\end{array}$} & \multirow{2}{*}{ COAnENTS } \\
\hline & & & & & 6 & 5 & H & & \\
\hline & & $s:=:=: 2$ & & & z=:z=: & & & & 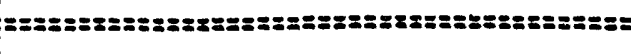 \\
\hline $740-6-236$ & $1-14$ & & 259 & Ift & & $\checkmark$ & & & \\
\hline-237 & 3 & & 250 & & & 1 & & & \\
\hline .233 & 3 & & 261 & $T$ & & 7 & & & \\
\hline-32 & -12 & & 32 & & & I & & & \\
\hline-24$)$ & 1 & & 253 & & & 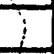 & & & \\
\hline$-240 A$ & $I$ & & 54 & & & I & & & \\
\hline-241 & 1 & & $\therefore 5$ & & & J & & & \\
\hline-242 & $I$ & & 266 & & & I & & & \\
\hline$-24 ?$ & ! & & 257 & & & T & & & \\
\hline-244 & $i$ & & 235 & & & 7 & & & \\
\hline-245 & - & & $26=$ & & & 1 & & & \\
\hline .245 & & & 270 & & & T & & & \\
\hline-24$)$ & & & 971 & & & - & & & \\
\hline-245 & 1 & & 272 & & & 1 & & & \\
\hline$-2^{4 a}$ & $\vdots$ & & 273 & & & is & & & \\
\hline-250 & $\because$ & & 274 & & & 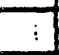 & & & \\
\hline$-250 . A$ & & & 275 & & & $!$ & & & \\
\hline-251 & I & & 276 & & & $!$ & & & \\
\hline .252 & & & 277 & & & & & & \\
\hline-253 & 1 & & 275 & & & 1 & & & \\
\hline .254 & 1 & & 270 & & & 1 & & & \\
\hline-255 & 1 & & 280 & $i$ & & 1 & & & \\
\hline-256 & & & 281 & $:$ & & $T$ & & & \\
\hline .257 & 1 & & 282 & & & $t$ & & & \\
\hline-258 & 1 & & 283 & & & $\overline{1}$ & & & \\
\hline-259 & $i$ & & 284 & & & 1 & & & \\
\hline-260 & $\therefore$ & & $2 x 5$ & & & 1 & & & \\
\hline $.60 A$ & $\vdots$ & & 236 & & & $j$ & & & \\
\hline .261 & $!$ & & $2 ; 9$ & ? & & $t$ & & & \\
\hline & & & & & & & & & \\
\hline & & & & & & & & & \\
\hline & & & & & & & & & \\
\hline & & & & & & & & & \\
\hline & & & & & & 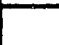 & & & \\
\hline & & & & & & & & & \\
\hline & & & & & & 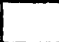 & & & \\
\hline & & & & & & & & & \\
\hline & & & & & & & & & \\
\hline & & & & & & & & & \\
\hline & & & & & & & & & \\
\hline & & & & & & & & & \\
\hline & & & & & & & & & \\
\hline
\end{tabular}


This page intentionally left blank. 


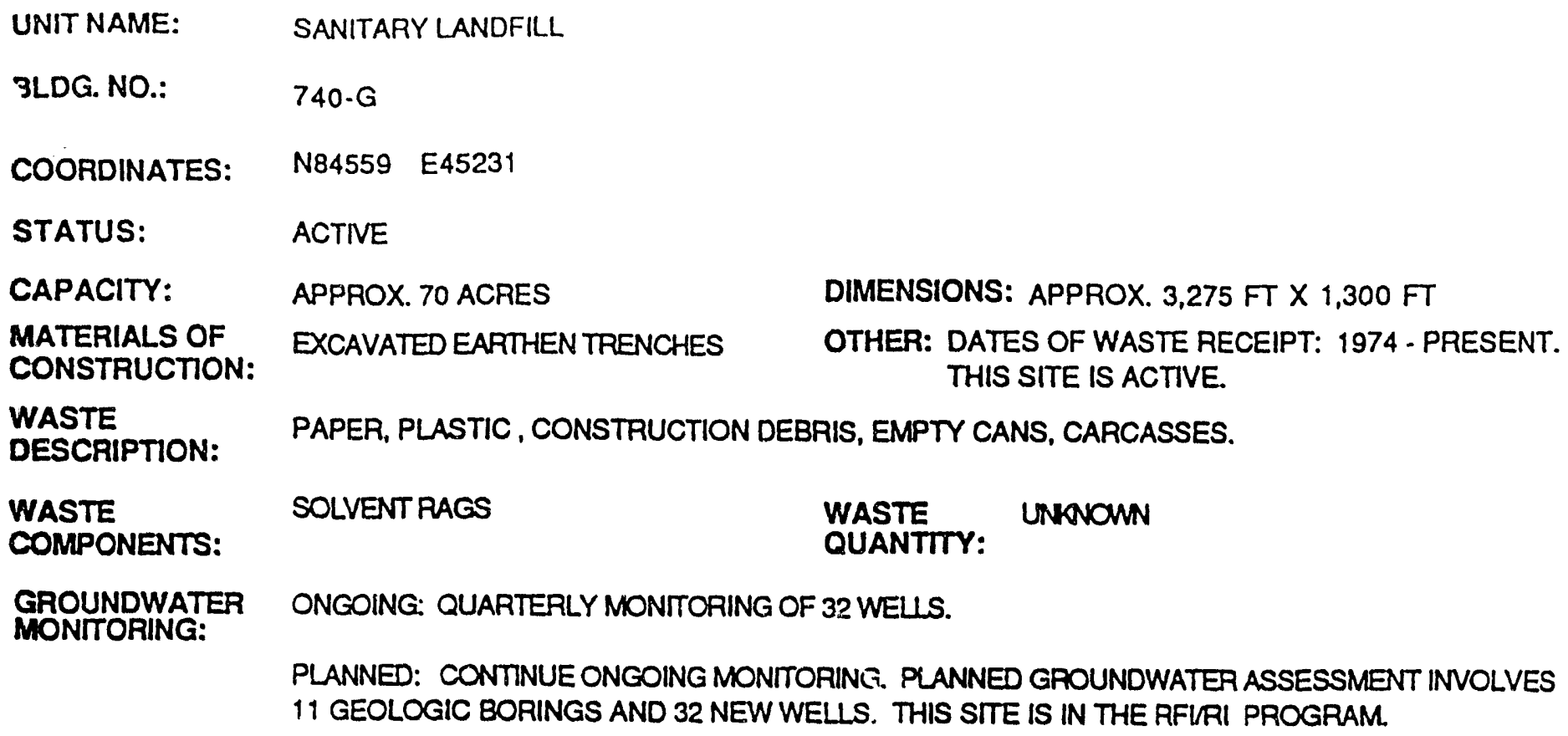

ONGOING: QUARTERLY MONTTORING OF 32 WEUS.

PLANNED: CONTINUE ONGOING MONITORING. PLANNED GROUNDWATER ASSESSMENT INVOLVES 11 GEOLOGIC BORINGS AND 32 NEW WELLS. THIS SITE IS IN THE RFI/RI PROGRAM.

WELLLDATA CODE: LFW (DPSPU-88-30-1, VOL II)

SUMMARY OF DATA:

¿.ARECTIVE ACTIONS:

INSPECTIONS AND FREQUENCY:
THIS SITE IS PERMITTED AS A SANITARY LANDFIL BY SCDHEC. HAZARDOUS WASTE DISPOSAL IS PROHIBITED BY PROCEDURE AND AS A PERMIT CONDITION. WASTE SEPARATION IS MADE AT THE STIE OF GENERATION. LOW LEVELS OF VOLATLE ORGANIC COMPOUNDS, INCLUDING TRICHLOROETHYLENE, TETRACHLOROETHYLENE, AND 1,1,1-TRICHLOROETHANE, HAVE BEEN DETECTED IN THE GROUNDWATER.

ONGOING: GROUNDWATERASSESSMENT.

PLANNED: THIS SITE IS IN THE RFI/RI PROGRAM. 
Thi: if loft blank. 

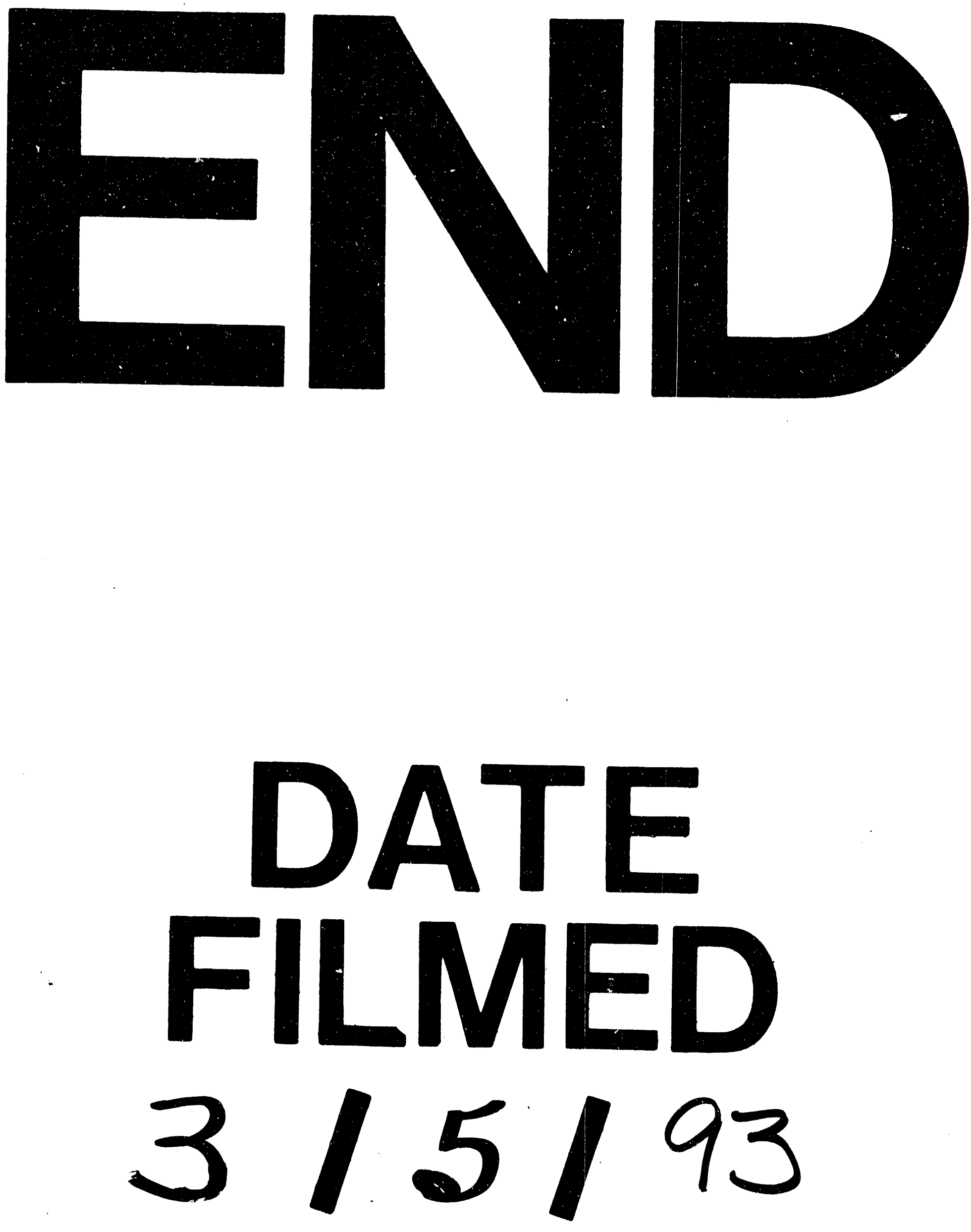